\title{
A Study of Double-Charm and Charm-Strange Baryons in Electron-Positron Annihilations
}

\author{
Adam J. Edwards
}

SLAC-R-883

Prepared for the Department of Energy under contract number DE-AC02-76SF00515

Printed in the United States of America. Available from the National Technical Information Service, U.S. Department of Commerce, 5285 Port Royal Road, Springfield, VA 22161. 
This document, and the material and data contained therein, was developed under sponsorship of the United States Government. Neither the United States nor the Department of Energy, nor the Leland Stanford Junior University, nor their employees, nor their respective contractors, subcontractors, or their employees, makes an warranty, express or implied, or assumes any liability of responsibility for accuracy, completeness or usefulness of any information, apparatus, product or process disclosed, or represents that its use will not infringe privately owned rights. Mention of any product, its manufacturer, or suppliers shall not, nor is it intended to, imply approval, disapproval, or fitness of any particular use. A royalty-free, nonexclusive right to use and disseminate same of whatsoever, is expressly reserved to the United States and the University. 


\title{
A STUDY OF DOUBLE-CHARM AND CHARM-STRANGE BARYONS IN ELECTRON-POSITRON ANNIHILATIONS
}

\author{
A DISSERTATION \\ SUBMITTED TO THE DEPARTMENT OF APPLIED PHYSICS \\ AND THE COMMITTEE ON GRADUATE STUDIES \\ OF STANFORD UNIVERSITY \\ IN PARTIAL FULFILLMENT OF THE REQUIREMENTS \\ FOR THE DEGREE OF \\ DOCTOR OF PHILOSOPHY
}

Adam Jacob Edwards

August 2007 
(C) Copyright by Adam Jacob Edwards 2007

All Rights Reserved 
I certify that I have read this dissertation and that, in my opinion, it is fully adequate in scope and quality as a dissertation for the degree of Doctor of Philosophy.

(Patricia Burchat) Principal Adviser

I certify that I have read this dissertation and that, in my opinion, it is fully adequate in scope and quality as a dissertation for the degree of Doctor of Philosophy.

(Vahe Petrosian)

I certify that I have read this dissertation and that, in my opinion, it is fully adequate in scope and quality as a dissertation for the degree of Doctor of Philosophy.

(Aaron Roodman)

Approved for the University Committee on Graduate Studies. 


\section{Abstract}

In this dissertation I describe a study of double-charm and charm-strange baryons based on data collected with the BABAR Detector at the Stanford Linear Accelerator Center. In this study I search for new baryons and make precise measurements of their properties and decay modes. I seek to verify and expand upon double-charm and charm-strange baryon observations made by other experiments. The BABAR Detector is used to measure subatomic particles that are produced at the PEP-II storage rings. I analyze approximately 300 million $e^{+} e^{-} \rightarrow c \bar{c}$ events in a search for the production of double-charm baryons. I search for the double-charm baryons $\Xi_{c c}^{+}$(containing the quarks $c c d)$ and $\Xi_{c c}^{++}(c c u)$ in decays to $\Lambda_{c}^{+} K^{-} \pi^{+}$and $\Lambda_{c}^{+} K^{-} \pi^{+} \pi^{+}$, respectively. No statistically significant signals for their production are found, and upper limits on their production are determined. Statistically significant signals for excited charm-strange baryons are observed with my analysis of approximately 500 million $e^{+} e^{-} \rightarrow c \bar{c}$ events. The charged charm-strange baryons $\Xi_{c}(2970)^{+}, \Xi_{c}(3055)^{+}, \Xi_{c}(3077)^{+}$, and $\Xi_{c}(3123)^{+}$are found in decays to $\Lambda_{c}^{+} K^{-} \pi^{+}$, the same decay mode used in the $\Xi_{c c}^{+}$search. The neutral charm-strange baryon $\Xi_{c}(3077)^{0}$ is observed in decays to $\Lambda_{c}^{+} K_{s} \pi^{-}$. I also search for excited charm-strange baryon decays to $\Lambda_{c}^{+} K_{s}, \Lambda_{c}^{+} K^{-}, \Lambda_{c}^{+} K_{s} \pi^{-} \pi^{+}$, and $\Lambda_{c}^{+} K^{-} \pi^{+} \pi^{-}$. No significant charm-strange baryon signals are found with these decay modes. For each excited charm-strange baryon state that I observe, I measure its mass, natural width (lifetime), and production rate. The properties of these excited charm-strange baryons and their decay modes provide constraints for phenomenological models of quark interactions through quantum chromodynamics. My discovery of the two new charm-strange baryons $\Xi_{c}(3055)^{+}$and $\Xi_{c}(3123)^{+}$influences our theoretical understanding of charm-strange baryon states. 


\section{Acknowledgments}

I will keep this short and sweet, just like the first person I would like to acknowledge. Amy Tang, you are the love of my life. I can't thank you enough for everything, because that's what you do for me, everything. I would like to thank my mother, father, and family for raising me to always try my best and teaching me to be crafty, intellectual, and independent. And I would like to thank and acknowledge Pat Burchat. She has been an endless source of support and guidance. But most important to me, from the very beginning, she has accepted me and welcomed me. To my friends and colleagues, thank you for the years of support and the years of fun. To everyone else, and you know who you are, and if you know who you are then you know what I'm saying, and if you know what I'm saying then I don't have to say it. 


\section{Contents}

Abstract $\quad$ v

Acknowledgments $\quad$ vii

1 Introduction $\quad 1$

1.1 The Electron-Positron Production of Hadrons . . . . . . . . . . . . . . . . . . . 2

1.2 Charm Baryons . . . . . . . . . . . . . . . . . . . . 3

1.3 Double-Charm and Excited Charm-Strange Baryons . . . . . . . . . . . . . 6

2 The BABAR Experiment $\quad 9$

2.1 The BABAR Detector . . . . . . . . . . . . . . . . . . . . . . 10

2.1.1 The Silicon Vertex Tracker _. . . . . . . . . . . . . . . . . . 12

2.1.2 The Drift Chamber . . . . . . . . . . . . . . . . . . . . . . . 12

2.1.3 The Cherenkov Ring-Imaging Detector . . . . . . . . . . . . . . . . . . . . 14

2.2 Data Acquisition and Triggering . . . . . . . . . . . . . . . . . 14

2.3 Particle Identification $\ldots \ldots \ldots \ldots \ldots$

$2.4 \quad$ BABAR Data Analysis . . . . . . . . . . . . . . . . . . . . . . 16

3 Double-Charm Baryons $r$

3.1 The Search Strategy . . . . . . . . . . . . . . . . . . . . . . . . 21

3.2 Data and Monte-Carlo Samples . . . . . . . . . . . . . . . . . . . . . . . 23

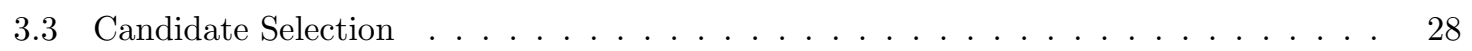

3.3 .1 Maximizing Significance . . . . . . . . . . . . . . . . . . 28

3.3.2 Optimization of Selection Criteria . . . . . . . . . . . . . . 29

3.3.3 Selection of $p^{*}$ Requirement . . . . . . . . . . . . . . . . . . 31

3.3.4 Multiple Candidates per Event . . . . . . . . . . . . . . . . 34

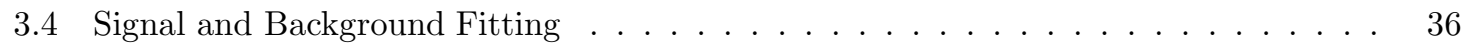

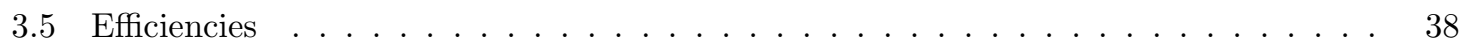

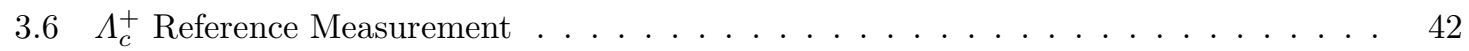


3.7 Search Statistics _ . . . . . . . . . . . . . . . . . . . . . . 44

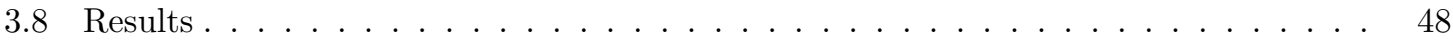

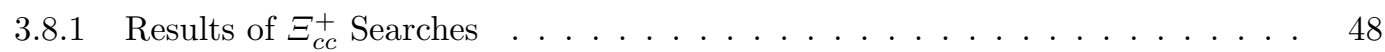

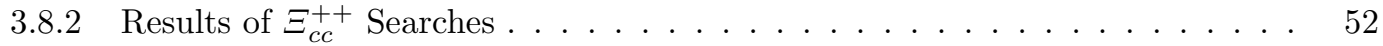

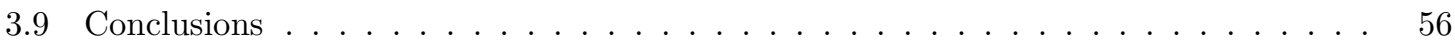

4 Charm-Strange Baryons $\quad 59$

4.1 Data and Monte-Carlo Samples . . . . . . . . . . . . . . . . . . . . . 60

4.2 Candidate Selection . . . . . . . . . . . . . . . . . . . . . 62

4.3 Three-Body Decays . . . . . . . . . . . . . . . . . . . . . . . . 64

4.3.1 Mass Resolution and Signal Efficiency . . . . . . . . . . . . . . . . . 68

4.3.2 Two-Dimensional PDF Components . . . . . . . . . . . . . . . . . . 73

4.3 .3 Likelihood Function . . . . . . . . . . . . . . . . . . . . . . . 80

4.3.4 Systematic Uncertainties . . . . . . . . . . . . . . . . . . . . . 85

4.3.5 Results for $\Lambda_{c}^{+} K^{-} \pi^{+} \ldots \ldots \ldots \ldots \ldots \ldots \ldots$

4.3.6 Results for $\Lambda_{c}^{+} K_{s} \pi^{-} \ldots \ldots \ldots \ldots \ldots \ldots \ldots$

4.4 Two-Body Decays _ . . . . . . . . . . . . . . . . . . . . . . . . 107

4.4.1 PDF Components and Likelihood Function . . . . . . . . . . . . . . . . 113

4.4.2 Mass Resolution and Signal Efficiency . . . . . . . . . . . . . . . . . . . . 118

4.4 .3 Data Results . . . . . . . . . . . . . . . . . . . . . . . . . . 121

4.5 Four-Body Decays . . . . . . . . . . . . . . . . . . . . . . . . 121

4.5.1 PDF Components and Likelihood Function _ . . . . . . . . . . . . 126

4.5.2 Mass Resolution and Signal Efficiency . . . . . . . . . . . . . . . . . . . 129

4.5 .3 Data Results . . . . . . . . . . . . . . . . . . . . . . . . . 132

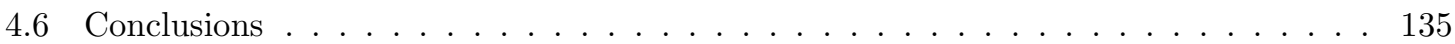

$\begin{array}{llr}5 & \text { Summary } & 139\end{array}$

$\begin{array}{ll}\text { Bibliography } & 141\end{array}$ 


\section{List of Tables}

1.1 Effective production cross-sections for $e^{+} e^{-} \rightarrow \gamma \rightarrow f \bar{f}$ at $10.58 \mathrm{GeV} \ldots \ldots \ldots 2$

3.1 Theoretical predictions for double-charm baryon and $c c$ diquark production cross-

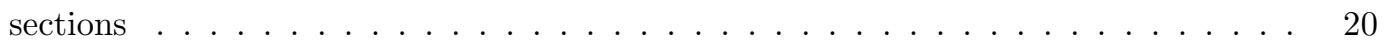

3.2 Variables used in the optimization of selection criteria $\ldots \ldots \ldots \ldots \ldots$

3.3 Levels of discrimination for optimal estimated significance of $\Xi_{c c}^{+}$in ranges of $p^{*} \ldots 33$

3.4 Levels of discrimination for optimal estimated significance of $\Xi_{c c}^{++}$in ranges of $p^{*} \ldots 33$

3.5 Final selection criteria chosen for double-charm baryon searches . . . . . . . . . . . 34

3.6 Fraction of events with multiple candidates and average number of candidates per event 35

3.7 The double-charm baryon signal shape parameters . . . . . . . . . . . . . . . . . . 39

3.8 Efficiencies from fragmentation-model MC samples. . . . . . . . . . . . . . . . . . . 42

3.9 Efficiencies as calculated from fragmentation-model MC samples and diquark-model MC samples . . . . . . . . . . . . . . . . . . . . . . . . 42

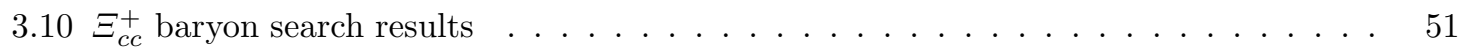

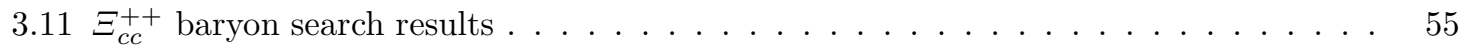

$3.1295 \%$ confidence-level upper limits on double-charm baryon $e^{+} e^{-}$production times

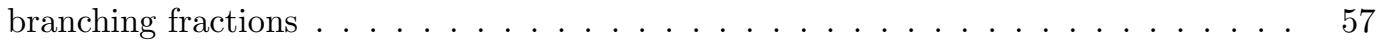

4.1 Simulated charm-strange baryon masses and natural widths . . . . . . . . . . . 61

4.2 Signal-MC samples for charm-strange baryons . . . . . . . . . . . . . . . . . . . 61

4.3 Invariant-mass side-band regions used to determine excited charm-strange baryon

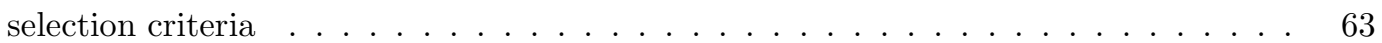

4.4 Selection criteria for excited charm-strange baryons . . . . . . . . . . . . . . 65

4.5 Candidates per event for $\Xi_{c}(2970)^{+, 0}$ and $\Xi_{c}(3077)^{+, 0}$ signal MC . . . . . . . . . 65

$4.6 M\left(\Lambda_{c}^{+} K_{s} \pi^{-}\right)$and $M\left(\Lambda_{c}^{+} K^{-} \pi^{+}\right)$mass resolutions for three-body decay modes $\ldots . \quad 68$

$4.7 M\left(\Lambda_{c}^{+} \pi^{-}\right)$and $M\left(\Lambda_{c}^{+} \pi^{+}\right)$mass resolutions for $\Xi_{c}(3077)^{+, 0}$ reconstruction $\ldots \ldots . \quad 69$

$4.8 M\left(\Lambda_{c}^{+} \pi^{-}\right)$and $M\left(\Lambda_{c}^{+} \pi^{+}\right)$mass resolutions for $\Xi_{c}(2970)^{0,+}$ reconstruction $\ldots \ldots .69$

4.9 Reconstruction efficiencies for $\Xi_{c}(2970)^{0}$ three-body decays $\ldots \ldots \ldots \ldots$. . . . . 70

4.10 Reconstruction efficiencies for $\Xi_{c}(3077)^{0}$ three-body decays $\ldots \ldots \ldots \ldots$. . . . 71 
4.11 Reconstruction efficiencies for $\Xi_{c}(2970)^{+}$three-body decays $\ldots \ldots \ldots$. . . . . . 71

4.12 Reconstruction efficiencies for $\Xi_{c}(3077)^{+}$three-body decays $\ldots \ldots \ldots$. . . . . . 72

4.13 Reconstruction efficiencies for $\Xi_{c}(3055)^{+, 0}$ and $\Xi_{c}(3123)^{+, 0}$ three-body decays . . . 72

4.14 Systematic uncertainties on masses, widths, yields, and fractions for states decaying

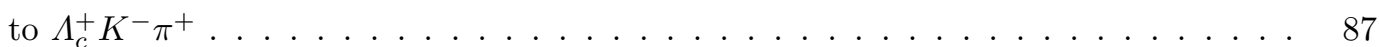

4.15 Systematic uncertainties on masses, widths, yields, and fractions for states decaying

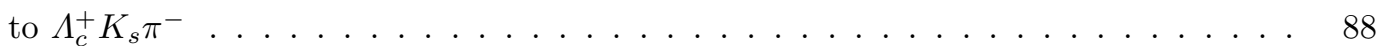

4.16 Masses, natural widths, yields, and significances of signals for $\Xi_{c}(2970)^{+}, \Xi_{c}(3055)^{+}$, $\Xi_{c}(3077)^{+}$, and $\Xi_{c}(3123)^{+}$decays to $\Lambda_{c}^{+} K^{-} \pi^{+} \ldots \ldots \ldots$. . . . . . . 91

4.17 Yields of $\Xi_{c}(2970)^{+}, \Xi_{c}(3055)^{+}, \Xi_{c}(3077)^{+}$, and $\Xi_{c}(3123)^{+}$signals by $\Lambda_{c}^{+}$decay mode 94

4.18 Signal yield ratios relative to the yields from the $\Lambda_{c}^{+} \rightarrow p K^{-} \pi^{+}$modes . . . . . . . 94

4.19 Production cross-sections times branching fractions for charm-strange baryons decaying to $\Lambda_{c}^{+} K_{s} \pi^{-}$and $\Lambda_{c}^{+} K^{-} \pi^{+} \ldots \ldots \ldots \ldots \ldots \ldots \ldots \ldots$

4.20 Masses, natural widths, yields, and significances of $\Lambda_{c}^{+} K_{s} \pi^{-}$signals $\ldots \ldots \ldots \ldots$

4.21 Yields for $\Xi_{c}(2970)^{0}$ and $\Xi_{c}(3077)^{0}$ three body decays $\ldots \ldots \ldots \ldots$. . . . . . 110

$4.22 M\left(\Lambda_{c}^{+} K_{s}\right)$ and $M\left(\Lambda_{c}^{+} K^{-}\right)$mass resolutions for two-body decay modes $\ldots \ldots \ldots$

4.23 Estimated efficiencies for two-body decay modes . . . . . . . . . . . . . . . 118

4.24 Results for two-body decay modes . . . . . . . . . . . . . . . . . . . . . 121

$4.25 M\left(\Lambda_{c}^{+} K_{s} \pi^{-} \pi^{+}\right)$and $M\left(\Lambda_{c}^{+} K^{-} \pi^{+} \pi^{-}\right)$mass resolutions for four-body decay modes . 129

4.26 Estimated efficiencies for four-body decay modes . . . . . . . . . . . . . . . . 132 


\section{List of Figures}

1.1 The hadronization process in an $e^{+} e^{-} \rightarrow c \bar{c}$ event . . . . . . . . . . . . 3

1.2 The mass spectrum of observed baryon states with at least one charm quark . . . . 5

1.3 A Feynman diagram of the weak decay $\Xi_{c c}^{+} \rightarrow \Lambda_{c}^{+} K^{-} \pi^{+} \ldots \ldots \ldots \ldots$

1.4 A Feynman diagram of a strongly decaying csu state . . . . . . . . . . . . . 8

2.1 Longitudinal section through the BABAR Detector . . . . . . . . . . . . . . . . . 11

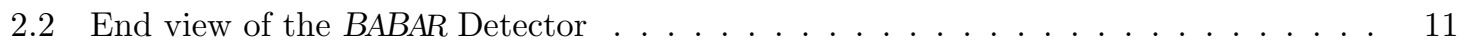

2.3 Longitudinal section through the SVT . . . . . . . . . . . . . . . . . . . 12

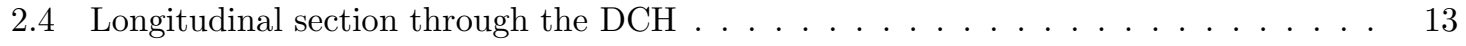

2.5 Schematic of a DIRC radiator bar and the DIRC imaging system . . . . . . . . . . . 14

2.6 Measured $\mathrm{d} E / \mathrm{d} x$ versus momentum as determined with the $\mathrm{DCH} \ldots \ldots \ldots$

2.7 Measured Cherenkov angle versus momentum for charged pion candidates . . . . . . 17

3.1 The mass-difference distributions for double-charm baryon searches . . . . . . . . . . 22

3.2 The $M\left(p K^{-} \pi^{+}\right)$invariant mass for $\Lambda_{c}^{+}$candidates from an integrated luminosity of

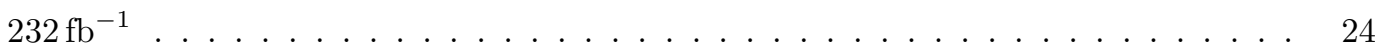

3.3 Simulated center-of-mass polar angle distributions of $\Xi_{c c}^{+} \ldots \ldots \ldots \ldots \ldots$

3.4 Simulated center-of-mass polar angle distributions of $\Xi_{c c}^{++} \ldots \ldots \ldots \ldots$

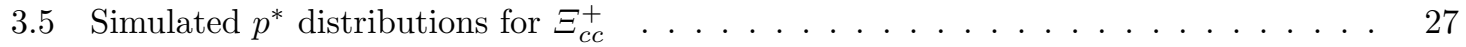

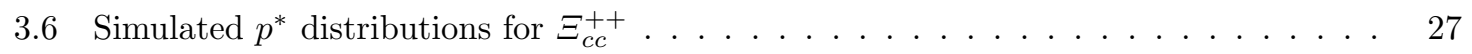

3.7 Distribution of $M\left(\left(p K^{-} \pi^{+}\right) K^{-} \pi^{+}\right)-M\left(p K^{-} \pi^{+}\right)$for all $\Xi_{c c}^{+}$candidates . . . . . 29

3.8 Distribution of $M\left(\left(p K^{-} \pi^{+}\right) K^{-} \pi^{+} \pi^{+}\right)-M\left(p K^{-} \pi^{+}\right)$for all $\Xi_{c c}^{++}$candidates . . . 30

3.9 Distributions of $p^{*}$ from side-band data of $\Xi_{c c}^{+}$candidates and truth-matched $\Xi_{c c}^{+} \mathrm{MC}$

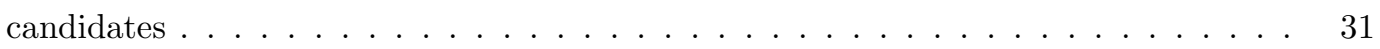

3.10 Distributions of $p^{*}$ from sideband-data $\Xi_{c c}^{++}$candidates and truth-matched $\Xi_{c c}^{++}$MC 32

3.11 Sets of selection criteria shown in a two-dimensional density plot of significance versus proton PID for $\Xi_{c c}^{+}$candidates . . . . . . . . . . . . . . 32

3.12 The estimated significance versus minimum allowed $p^{*}$ for $\Xi_{c c}^{+}$using the diquark-model 35 3.13 The estimated significance versus minimum allowed $p^{*}$ for $\Xi_{c c}^{++}$using the diquark-model 35 
3.14 The estimated significance versus minimum allowed $p^{*}$ for $\Xi_{c c}^{+}$using the fragmentationmodel and side-band data . . . . . . . . . . . . . . . . . 35

3.15 The estimated significance versus minimum allowed $p^{*}$ for $\Xi_{c c}^{++}$using the fragmentationmodel and side-band data . . . . . . . . . . . . . . . . . . 35

3.16 Signal-MC $\Xi_{c c}^{+}$in the two-dimensional $\Delta M$ vs. $M_{\Lambda_{c}}$ signal region $\ldots \ldots \ldots \ldots$. . . 37

3.17 Signal-MC $\Xi_{c c}^{++}$in the two-dimensional $\Delta M$ vs. $M_{\Lambda_{c}}$ signal region $\ldots \ldots \ldots \ldots$

3.18 Invariant-mass distributions of $\Xi_{c c}^{+}$candidates with no $p^{*}$ requirement $\ldots \ldots$. . . . 39

3.19 Invariant-mass distributions of $\Xi_{c c}^{+}$candidates with $p^{*}>2.3 \mathrm{GeV} / c^{2} \ldots \ldots \ldots \ldots$

3.20 Invariant-mass distributions of $\Xi_{c c}^{++}$candidates with no $p^{*}$ requirement . . . . . . 40

3.21 Invariant-mass distributions of $\Xi_{c c}^{++}$candidates with $p^{*}>2.3 \mathrm{GeV} / c^{2} \ldots \ldots \ldots$

3.22 One dimensional fit of $M\left(p K^{-} \pi^{+}\right)$invariant-mass distribution with no $p^{*}$ requirement 43

3.23 One dimensional fit of $M\left(p K^{-} \pi^{+}\right)$invariant-mass distribution with $p^{*}>2.3 \mathrm{GeV} / c$

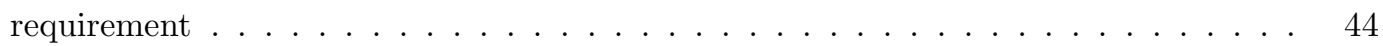

3.24 Distribution of significance measures from searches performed on $1000 \mathrm{TCM}$ samples for $\Xi_{c c}^{++}$signal within $5 \mathrm{MeV} / c^{2}$ of $3460 \mathrm{MeV} / c^{2} \ldots \ldots \ldots \ldots$

3.25 Distribution of significance measures from searches performed on $1000 \mathrm{TMC}$ samples for $\Xi_{c c}^{+}$signal within the $210 \mathrm{MeV} / c^{2}$ invariant-mass search region and with $p^{*}>$

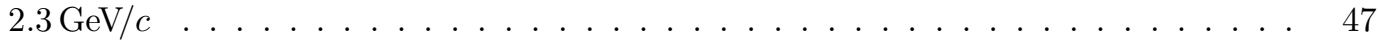

3.26 Distribution of significance measures from $\Xi_{c c}^{++}$with signal mean and width fixed . . 47

$3.27 M_{\Lambda_{c}}$ and $\Delta M$ invariant-mass projections and the fit for $\Xi_{c c}^{+}$signal within $5 \mathrm{MeV} / c^{2}$

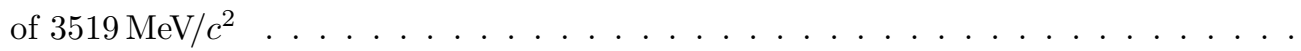

$3.28 M_{\Lambda_{c}}$ and $\Delta M$ invariant-mass projections and the fit for $\Xi_{c c}^{+}$signal within $5 \mathrm{MeV} / c^{2}$ of $3519 \mathrm{MeV} / c^{2}$ and $p^{*}>2.3 \mathrm{GeV} / c^{2} \ldots \ldots \ldots \ldots \ldots \ldots$

$3.29 M_{\Lambda_{c}}$ and $\Delta M$ invariant-mass projections for the most significant fit to the $\Xi_{c c}^{+}$data 50

$3.30 M_{\Lambda_{c}}$ and $\Delta M$ invariant-mass projections for the most significant fit to the $\Xi_{c c}^{+}$data

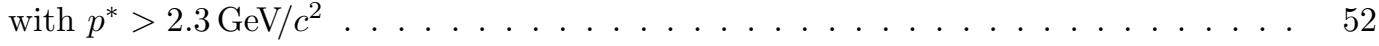

$3.31 M_{\Lambda_{c}}$ and $\Delta M$ invariant-mass projections and the fit for $\Xi_{c c}^{++}$signal within $5 \mathrm{MeV} / c^{2}$

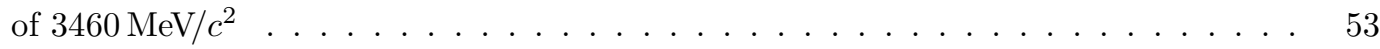

$3.32 M_{\Lambda_{c}}$ and $\Delta M$ invariant-mass projections and the fit for $\Xi_{c c}^{++}$signal within $5 \mathrm{MeV} / c^{2}$ of $3460 \mathrm{MeV} / c^{2}$ and $p^{*}>2.3 \mathrm{GeV} / c^{2} \ldots \ldots \ldots \ldots \ldots \ldots \ldots$

$3.33 M_{\Lambda_{c}}$ and $\Delta M$ invariant-mass projections for the most significant fit to the $\Xi_{c c}^{++}$data 54

$3.34 M_{\Lambda_{c}}$ and $\Delta M$ invariant-mass projections for the most significant fit to the $\Xi_{c c}^{++}$data with $p^{*}>2.3 \mathrm{GeV} / c^{2} \ldots \ldots \ldots \ldots \ldots \ldots \ldots$

4.1 Dalitz plot of $M\left(\pi^{+} K^{-}\right)^{2}$ versus $M\left(\Lambda_{c}^{+} \pi^{+}\right)^{2}$ for $\Xi_{c}(2970)^{+}$candidates in data . . $\quad 67$

4.2 Dalitz plot of $M\left(\pi^{+} K^{-}\right)^{2}$ versus $M\left(\Lambda_{c}^{+} \pi^{+}\right)^{2}$ for $\Xi_{c}(3077)^{+}$candidates in data . . $\quad 67$

4.3 Dalitz plot of $M\left(\pi^{-} K_{s}\right)^{2}$ versus $M\left(\Lambda_{c}^{+} \pi^{-}\right)^{2}$ for $\Xi_{c}(2970)^{0}$ candidates in data . . . 67

4.4 Dalitz plot of $M\left(\pi^{-} K_{s}\right)^{2}$ versus $M\left(\Lambda_{c}^{+} \pi^{-}\right)^{2}$ for $\Xi_{c}(3077)^{0}$ candidates in data . . . 67 
4.5 Reconstructed invariant-mass distributions for $\Lambda_{c}^{+}$candidates in data . . . . . . . . 74

4.6 $M_{\Xi_{c}}$ projections of $\Lambda_{c}^{+}$-sideband $\Lambda_{c}^{+} K_{s} \pi^{-}$data fit with a threshold function . . . . 75

$4.7 M_{\Sigma_{c}}$ projections of $\Lambda_{c}^{+}$-sideband $\Lambda_{c}^{+} K_{s} \pi^{-}$data fit with a threshold function $\ldots . .76$

$4.8 M_{\Xi_{c}}$ projections of wrong-sign $\Lambda_{c}^{+} K^{+} \pi^{-}$candidates in data fit with the sum of nonresonant and resonant PDF background components . . . . . . . . . . . . 78

$4.9 M_{\Sigma_{c}}$ projections of wrong-sign $\Lambda_{c}^{+} K^{+} \pi^{-}$candidates in data fit with the sum of nonresonant and resonant PDF background components . . . . . . . . . . . . . . . 79

$4.10 M_{\Xi_{c}}$ projections for generic- $c \bar{c} \Lambda_{c}^{+} K_{s} \pi^{-}$MC candidates combined with nonresonant signal TMC, and an illustration of the nonresonant signal PDF components . . . . .

$4.11 M_{\Sigma_{c}}$ projections for generic-c $\bar{c} \Lambda_{c}^{+} K_{s} \pi^{-}$MC candidates combined with signal TMC, and an illustration of the nonresonant signal PDF components . . . . . . . . . .

$4.12 M_{\Xi_{c}}$ projections for generic-c $\bar{c} \Lambda_{c}^{+} K^{-} \pi^{+}$MC candidates combined with signal TMC, and an illustration of the resonant signal PDF components . . . . . . . . . . . 83

$4.13 M_{\Sigma_{c}}$ projections for generic- $c \bar{c} \Lambda_{c}^{+} K^{-} \pi^{+}$MC candidates combined with signal TMC, and an illustration of the resonant signal PDF components . . . . . . . . . . . . 84

$4.14 M\left(\Lambda_{c}^{+} K^{-} \pi^{+}\right)$projections of $\Lambda_{c}^{+} K^{-} \pi^{+}$candidates in data and their fit . . . . . . . 92

$4.15 M\left(\Lambda_{c}^{+} \pi^{+}\right)$projections of $\Lambda_{c}^{+} K^{-} \pi^{+}$candidates in data and their fit . . . . . . . . . 93

4.16 Two-dimensional normalized residuals for reconstructed $\Lambda_{c}^{+} K^{-} \pi^{+}$candidates in data and their fit ........................... 95

$4.17 M_{\Xi_{c}}$ projections of $\Lambda_{c}^{+} K^{-} \pi^{+}$candidates in data with a fit of $\Xi_{c}(2970)^{+}, \Xi_{c}(3055)^{+}$, $\Xi_{c}(3077)^{+}$, and $\Xi_{c}(3123)^{+} \ldots \ldots \ldots \ldots$. . . . . . . . . . . . . 96

4.18 Scatter plot of $\Lambda_{c}^{+} K^{-} \pi^{+}$candidates in data . . . . . . . . . . . . . . . . . . . . . 97

4.19 Scatter plot of wrong-sign $\Lambda_{c}^{+} K^{+} \pi^{-}$data . . . . . . . . . . . . . . . . . . 97

$4.20 M_{\Xi_{c}}$ projections of wrong-sign $\Lambda_{c}^{+} K^{+} \pi^{-}$data with background PDF fits . . . . . . 98

4.21 Scatter plot of the $\Lambda_{c}^{+} K^{-} \pi^{+}$reconstructed generic-c $\bar{c}$ MC sample . . . . . . . . . . . 99

4.22 Scatter plot of the data with $M_{\Xi_{c}}$ recalculated as $M\left(\Lambda_{c}^{+} \pi^{-} \pi^{+}\right)$. . . . . . . . . . 100

$4.23 M_{\Xi_{c}}$ projections of the $\Lambda_{c}^{+} K^{-} \pi^{+}$reconstructed generic-c $\bar{c}$ MC sample with background $\mathrm{PDF}$ fits . . . . . . . . . . . . . . . . . . . . . 101

$4.24 M_{\Xi_{c}}$ projections of the data where $M_{\Xi_{c}}$ has been recalculated as $M\left(\Lambda_{c}^{+} \pi^{-} \pi^{+}\right) \quad$. . 102

$4.25 M_{\Xi_{c}}$ projections of $\Lambda_{c}^{+} K^{-} \pi^{+}$candidates with $p^{*}>3.5$ and their fit . . . . . . . . 104

$4.26 M_{\Xi_{c}}$ projections of $\Lambda_{c}^{+} K^{-} \pi^{+}$candidates with $p^{*}>4.0$ and their fit . . . . . . . 105

$4.27 M\left(\Lambda_{c}^{+} K_{s} \pi^{-}\right)$projections of $\Lambda_{c}^{+} K_{s} \pi^{-}$candidates in data and their fit . . . . . . . . 108

$4.28 M\left(\Lambda_{c}^{+} \pi^{-}\right)$projections of $\Lambda_{c}^{+} K_{s} \pi^{-}$candidates in data and their fit . . . . . . . . . 109

4.29 Two-dimensional pulls for reconstructed $\Lambda_{c}^{+} K_{s} \pi^{-}$candidates in data and their fit . . 110

$4.30 M\left(\Lambda_{c}^{+} K_{s} \pi^{-}\right)$projections of $\Lambda_{c}^{+} K_{s} \pi^{-}$candidates in data and their fit with background and $\Xi_{c}(3077)^{0}$ PDF components . . . . . . . . . . . . . . . . . . 111 
$4.31 M\left(\Lambda_{c}^{+} \pi^{-}\right)$projections of $\Lambda_{c}^{+} K_{s} \pi^{-}$candidates in data and their fit with background

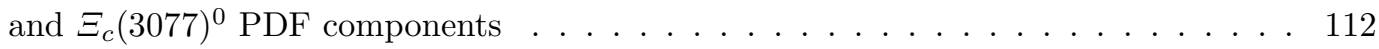

4.32 Two-dimensional pulls for reconstructed $\Lambda_{c}^{+} K_{s} \pi^{-}$candidates in data and their fit . . 113

4.33 Invariant-mass distributions of wrong-sign $\Lambda_{c}^{+} K^{+}$candidates $\ldots \ldots \ldots \ldots \ldots$

4.34 Invariant-mass distributions of $\Lambda_{c}^{+} K_{s}$ candidates with $\Lambda_{c}^{+}$mass in the sideband range 116

4.35 Invariant-mass distributions of $\Lambda_{c}^{+} K^{-}$candidates with $\Lambda_{c}^{+}$mass in the sideband range 117

4.36 Unbinned likelihood fits to signal-MC $\Xi_{c}(2970)^{+} \rightarrow \Lambda_{c}^{+} K_{s}$ candidates used for estimating efficiencies . . . . . . . . . . . . . . . . . . . . . . 119

4.37 Unbinned likelihood fits to signal-MC $\Xi_{c}(3077)^{+} \rightarrow \Lambda_{c}^{+} K_{s}$ candidates used for estimating efficiencies . . . . . . . . . . . . . . . . 120

$4.38 \Lambda_{c}^{+} K_{s}$ candidates and fit with a PDF component for a $\Xi_{c}(2970)^{+}$signal . . . . . . 122

$4.39 \Lambda_{c}^{+} K_{s}$ candidates and fit with a PDF component for a $\Xi_{c}(3077)^{+}$signal $\ldots \ldots \ldots$

$4.40 \Lambda_{c}^{+} K^{-}$candidates and fit with a PDF component for a $\Xi_{c}(2970)^{0}$ signal . . . . . . . 124

$4.41 \Lambda_{c}^{+} K^{-}$candidates and fit with a PDF component for a $\Xi_{c}(3077)^{0}$ signal . . . . . . 125

4.42 Relative four-body phase-space as a function of the $\Lambda_{c}^{+} K_{s} \pi^{+} \pi^{-}$invariant mass $\ldots 126$

4.43 Relative four-body phase-space as a function of the $\Lambda_{c}^{+} K^{-} \pi^{+} \pi^{-}$invariant mass . . . 126

4.44 Invariant-mass distributions of wrong-sign $\Lambda_{c}^{+} K^{+} \pi^{-} \pi^{-}$candidates $\ldots \ldots \ldots \ldots$

4.45 Invariant-mass distributions of $\Lambda_{c}^{+}$side-band $\Lambda_{c}^{+} K^{-} \pi^{-} \pi^{+}$candidates . . . . . . . 128

4.46 Mass resolutions for $\Xi_{c}(3077)^{+} \rightarrow \Lambda_{c}^{+} K_{s} \pi^{-} \pi^{+} \ldots \ldots \ldots \ldots \ldots$. . . . . 130

4.47 Mass resolutions for $\Xi_{c}(3077)^{0} \rightarrow \Lambda_{c}^{+} K^{-} \pi^{+} \pi^{-} \ldots \ldots \ldots \ldots \ldots \ldots$. . . . . 131

4.48 Unbinned likelihood fits to signal-MC $\Xi_{c}(3077)^{+} \rightarrow \Lambda_{c}^{+} K_{s} \pi^{+} \pi^{-}$candidates used for

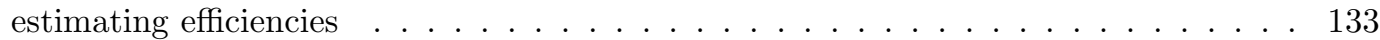

4.49 Unbinned likelihood fits to signal-MC $\Xi_{c}(3077)^{0} \rightarrow \Lambda_{c}^{+} K^{-} \pi^{+} \pi^{-}$candidates used for estimating efficiencies . . . . . . . . . . . . . . . . . 134

4.50 Signal PDF component for $\Xi_{c}(3077)^{+} \rightarrow \Lambda_{c}^{+} K_{s} \pi^{-} \pi^{+} \ldots \ldots \ldots \ldots \ldots \ldots$

$4.51 M\left(\Lambda_{c}^{+} K_{s} \pi^{-} \pi^{+}\right)$invariant-mass distributions and their fit for $\Xi_{c}(3077)^{+}$signal $\ldots 136$

$4.52 M\left(\Lambda_{c}^{+} K^{-} \pi^{+} \pi^{-}\right)$invariant-mass distributions and their fit for $\Xi_{c}(3077)^{0}$ signal $\ldots 137$ 


\section{Chapter 1}

\section{Introduction}

In this dissertation I describe a study of both double-charm and charm-strange baryons produced from positron-electron $\left(e^{+} e^{-}\right)$annihilations in the BABAR Detector at the Stanford Linear Accelerator Center (SLAC). This study is motivated by my desire to investigate new particles and to test theoretical models of particle physics. Our understanding of the production and decay of baryons is based on the Standard Model of elementary particle physics. As part of the Standard Model, quantum chromodynamics (QCD) provides a framework for predicting what types of quark states can form, and their production and strong decay rates. QCD does not lead to exact calculations, but models are developed to approximate its effects and to make theoretical predictions. Measurements of particle masses, production rates, decay rates, and decay modes can be compared to theoretical predictions. These comparisons can be used to identify the quantum states of observed baryons. Such comparisons can also be used to evaluate the validity of different theoretical models. Finding new states and new decay modes, and measuring production and strong-decay rates provides tests of phenomenological models of quark interactions in QCD. This dissertation includes searches for the $e^{+} e^{-}$production of double-charm and excited charm-strange baryons, measurements of production rates, measurements of masses and decay rates, and searches for new decay modes.

Theoretically, the lowest mass double-charm baryons are the $\Xi_{c c}^{+}$(containing the quarks $c c d$ ) and the $\Xi_{c c}^{++}(c c u)$; both have spin-parity $J^{P}=(1 / 2)^{+}$. Throughout this dissertation, whenever I express a particle or decay mode, the charge conjugate is also implied. A charm hadroproduction experiment at Fermilab has reported evidence for both the $\Xi_{c c}^{+}$and the $\Xi_{c c}^{++}[1,2,3]$, but other experiments have not been able to verify the existence of these states $[4,5,6]$. This dissertation includes a search for $e^{+} e^{-}$production of $\Xi_{c c}^{+}$and $\Xi_{c c}^{++}$. No statistically significant signals for double-charm baryons are found, and upper limits on their production rates are determined.

The $e^{+} e^{-}$production of charm-strange baryons is a well-documented phenomenon. The three charm-strange baryon ground states have previously been observed [7]: the $\Xi_{c}$, the $\Xi_{c}^{\prime}$, and the $\Xi_{c}(2635)$ with spin-parities $(1 / 2)^{+},(1 / 2)^{+}$, and $(3 / 2)^{+}$, respectively. A few charm-strange states 
Table 1.1: Production cross-sections for $e^{+} e^{-} \rightarrow \gamma \rightarrow f \bar{f}$ at $10.58 \mathrm{GeV}$.

\begin{tabular}{cccccccc}
\hline$e^{+} e^{-} \rightarrow$ & $b \bar{b}$ & $c \bar{c}$ & $s \bar{s}$ & $u \bar{u}$ & $d \bar{d}$ & $\tau^{-} \tau^{+}$ & $\mu^{-} \mu^{+}$ \\
\hline \hline Cross-Section (nb) & 1.05 & 1.30 & 0.35 & 1.30 & 0.35 & 0.94 & 1.16 \\
\hline \hline
\end{tabular}

with radial or orbital excitations have been observed. In particular, three baryons called $\Xi_{c}(2980)^{+}$, $\Xi_{c}(3077)^{+}$, and $\Xi_{c}(3077)^{0}$ have been recently observed [6]. The studies described in this dissertation verify the $e^{+} e^{-}$production of these three baryons and also show the existence of two other excited charm-strange baryons, $\Xi_{c}(3055)^{+}$and $\Xi_{c}(3123)^{+}$. The spin-parities of the five studied charmstrange baryons are unknown, but theory suggests that they are radial and/or orbital excitations of the previously observed lower mass states. The masses, widths, and production rates of these excited charm-strange baryons are measured and several possible decay modes are studied.

The following three sections provide an introduction to the study of double-charm and charmstrange baryons. Chapter 2 of this dissertation describes the BABAR Experiment in some detail. The description of the data analysis is divided into two chapters. Chapter 3 details the search for doublecharm baryons and Chapter 4 details the study or excited charm-strange baryons. A summary and discussion of the dissertation is given in Chapter 5 .

\subsection{The Electron-Positron Production of Hadrons}

The PEP-II storage rings are operated at SLAC and produce $e^{+} e^{-}$annihilations at a center-of-mass energy of $10.58 \mathrm{GeV}$. This energy corresponds to the mass of the $\Upsilon(4 S)$ resonance, which contains both a $b$ (bottom) and a $\bar{b}$ quark, and decays predominantly to a pair of $B$ mesons. The production of $B$-meson pairs is the primary goal of PEP-II, and the primary objective of the BABAR Collaboration is to study decays of $B$ mesons. Even at the $\Upsilon(4 S)$ resonance, however, it is impossible to create only $b$ quarks, and several other kinds of fermion pairs are also produced in large numbers through the process $e^{+} e^{-} \rightarrow \gamma \rightarrow f \bar{f}$. At $10.58 \mathrm{GeV}$, the effective $e^{+} e^{-}$production cross-sections for $b \bar{b}, c \bar{c}$, $\tau^{-} \tau^{+}$, and other particles are similar in magnitude as shown in Table 1.1. Initial state radiation, where the electron or positron radiates a high energy photon before their mutual annihilation, leads to $e^{+} e^{-}$annihilations at energies below $10.58 \mathrm{GeV}$. These lower-energy annihilations are also studied by the BABAR Collaboration. The high luminosity provided by PEP-II has resulted in the creation of hundreds of millions of "events" where an electron and positron annihilate and these are studied extensively by the BABAR Collaboration.

The BABAR Collaboration designed the BABAR Detector [10] to fit around the $e^{+} e^{-}$interaction region of PEP-II. The BABAR Detector is composed of several subsystems that each serve to measure different aspects of the high-energy particles created in an $e^{+} e^{-}$event. These particles nominally 


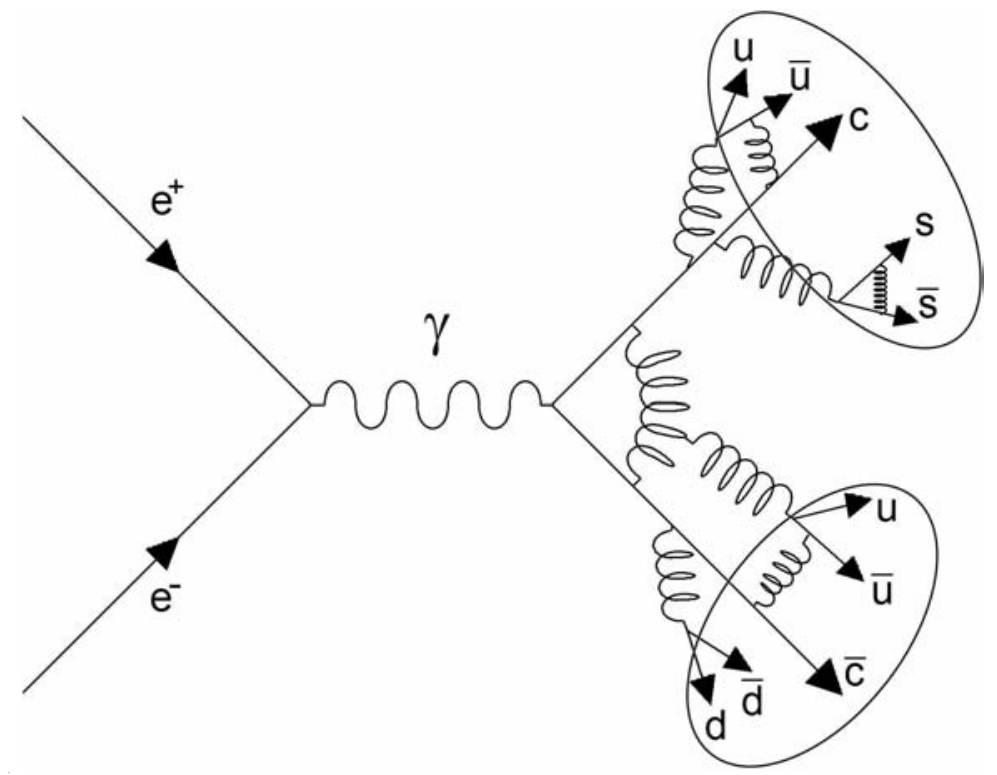

Figure 1.1: A Feynman diagram of an $e^{+} e^{-} \rightarrow c \bar{c}$ event. The curled lines represent gluons, which produce quark anti-quark pairs and mediate the hadronization process. The two ovals indicate the two jets of hadrons being formed in the direction of the initial $c$ and $\bar{c}$ quarks.

originate directly from the PEP-II $e^{+} e^{-}$events or from the decay of the particles produced by these events. The $e^{+} e^{-}$events most pertinent to my research are those that produce a $c \bar{c}$ quark pair. With a total energy of $10.58 \mathrm{GeV}$, the charm quark and anti-quark are produced with high momenta. They rapidly form hadrons through a QCD process known as hadronization. I illustrate the beginnings of such a $c \bar{c}$-hadronization process in Figure 1.1. During the hadronization process, the gluon mediators of the QCD force produce additional quark anti-quark pairs, which combine with the original $c \bar{c}$ to form color-neutral hadrons. These hadrons form in two groups, called jets, each with total momenta approximately equal to those of the original $c$ and $\bar{c}$. The types of hadrons formed in the jets are dependent on the details of QCD. With the production of an additional $c \bar{c}$ pair in the hadronization process, it may be possible for a double-charm baryon to form. The BABAR Collaboration and other experiments have previously shown that $s \bar{s}$ production in the hadronization process can lead to charm-strange baryons [7]. Through the hadronization process, QCD dictates what types and excitations of charm-strange baryons are formed and at what rates.

\subsection{Charm Baryons}

In Figure 1.2 I illustrate the mass spectrum of observed baryon states with one or two charm quarks $[2,7,8,9]$. The states in the $\Lambda_{c}$ and $\Sigma_{c}$ columns are composed of $c, u$, and $d$ quarks. The $u$ and $d$ quarks are nearly mass degenerate and can be considered to have an $\mathrm{SU}(2)$ flavor 
symmetry known as isospin. With this same flavor symmetry, the $\Sigma_{c}$ are symmetric isospin triplet states $(u u),(u d+d u)$, and $(d d)$ with total isospin 1 , while the $\Lambda_{c}^{+}$are anti-symmetric isospin singlet states $(u d-d u)$ with total isospin 0. Therefore, each $\Lambda_{c}$ state indicated in Figure 1.2 represents just one baryon, while each $\Sigma_{c}$ state represents three baryons with different electromagnetic charge $(++,+, 0)$ but nearly identical mass. The $\Sigma_{c}$ baryon masses are not exactly identical because the $u-$ and $d$-quark masses and charges are different. Each $\Xi_{c}$ state indicated in Figure 1.2 represents two baryons with quark content csu (electromagnetic charge + ) or csd (electromagnetic charge 0 ). The states in the $\Xi_{c}$ column are isospin doublets with total isospin $1 / 2$. The $\Omega_{c}$ states have isospin 0 (no $u$ or $d$ quark) and represent individual baryons with css quark content and zero electromagnetic charge.

The mass spectrum of states in each column of Figure 1.2 is due to different configurations or excitations of the constituent quarks. The spin and orbital configuration of each state can be summarized by its spin-parity, $J^{P}$, where $J$ is the total angular momentum of the state and $P$ is its parity. Each quark carries a spin angular momentum of $1 / 2$. These quark spins can be combined to give $J=1 / 2$ or $J=3 / 2$. With the addition of one unit of orbital angular momentum, the total angular momentum can be $J=1 / 2, J=3 / 2$, or $J=5 / 2$. By definition, every quark has a parity of + and every anti-quark has a parity of - . Combining parity is a multiplicative process, which makes the combined parity of the three quarks in a baryon + and the three anti-quarks of an anti-baryon -. For every unit of orbital angular momentum that the three quarks or anti-quarks might have, there is another factor of - in their combined parity. The spin-parity of the different states given in Figure 1.2 are based on quark-model predictions of masses for states with different spin-parity (their spin-parity have not been directly measured). The mass differences in each column of Figure 1.2 are primarily determined by spin and orbital angular momentum. In general, states with larger combined spin or larger orbital angular momentum are more massive. A radial excitation of the quarks in a baryon will also result in a state with higher mass. Some of the higher mass $\Lambda_{c}$ and $\Xi_{c}$ states might be examples of states with a radial excitation.

Ground states have no orbital or radial excitations. As can be seen in Figure 1.2, there is only one ground state $\Lambda_{c}^{+}$and it has spin-parity $(1 / 2)^{+}$. There are two $\Sigma_{c}$ ground states with spinparities $(1 / 2)^{+}$and $(3 / 2)^{+}$. The lack of a $\Lambda_{c}^{+}$ground state with spin-parity $(3 / 2)^{+}$is due to Fermi statistics, which requires the color-space-spin-flavor wave-function state to be anti-symmetric under the interchange of the two light quarks $(u$ and $d)$. When dealing with ground-state baryons with no orbital angular momentum, the spatial part of the wave function is symmetric. QCD requires that the color wave function be antisymmetric. This leaves the spin-flavor wave function of ground-state baryons to be symmetric under the interchange of the two light quarks. The $\Lambda_{c}^{+}$corresponds to a flavor anti-symmetric singlet state and the $\Sigma_{c}$ to a flavor symmetric triplet state. These flavor symmetries require the spin symmetry of the $\Lambda_{c}^{+}$state to be odd and the spin symmetry of the $\Sigma_{c}$ states to be even under the interchange of $u$ and $d$. Combining the three spin- $1 / 2$ quarks $c, u$, 


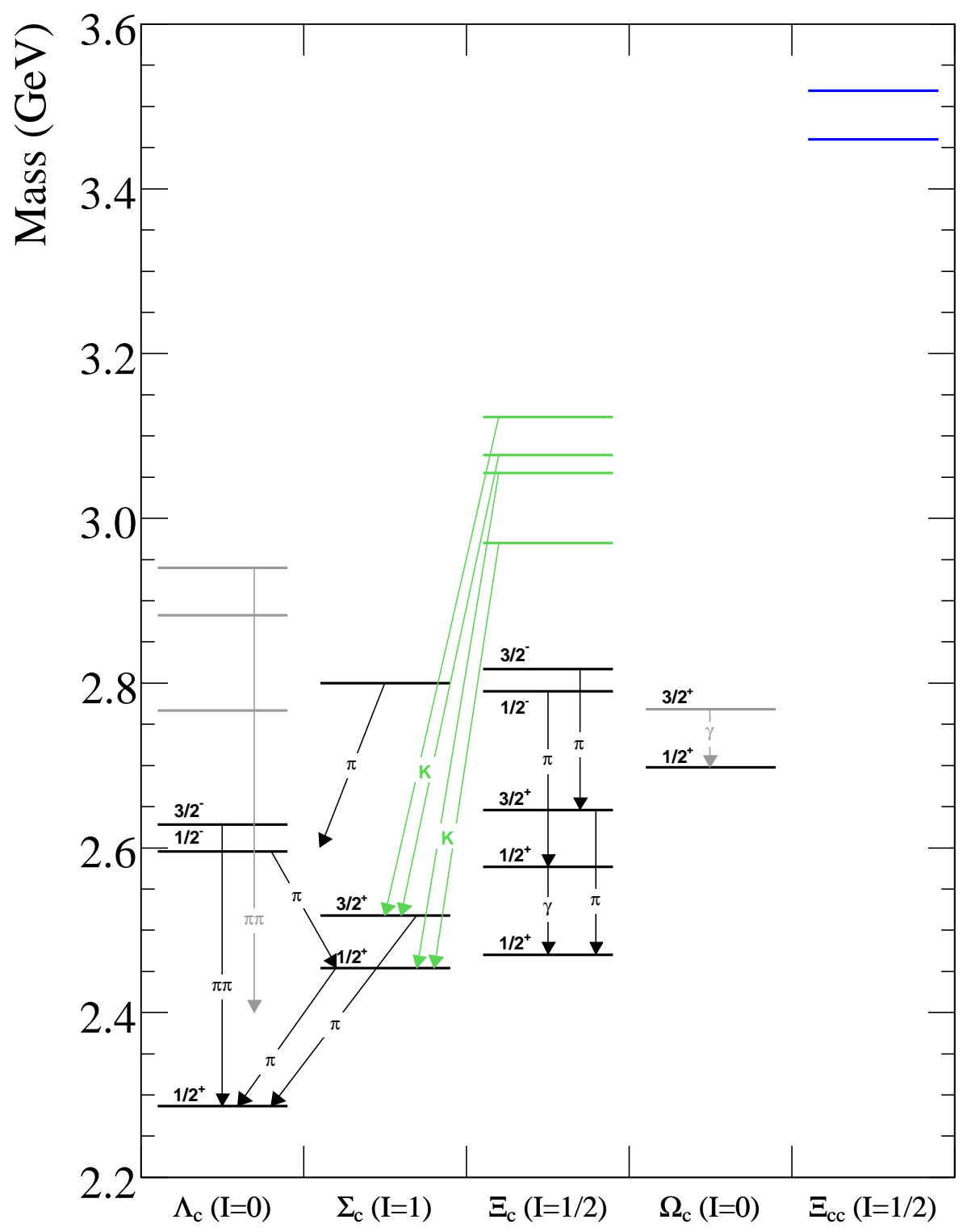

Figure 1.2: The mass spectrum of observed baryon states with at least one charm quark (horizontal lines) and some of their strong and electromagnetic decay modes (arrows). Each arrow points from the original parent state to the daughter charm-baryon state produced in the decay, and each is labeled by the daughter mesons or photon produced in the decay. The spin-parities $\left(J^{P}\right)$ of states are given for cases in which they have been theoretically identified. Well-established states are shown in black, and less well-established states are shown in gray. The excited charm-strange baryon states studied in this dissertation are illustrated in green, and the double-charm baryon states (which are not verified by this study) are shown in blue. Each column is labeled by the name of the states in that column and their isospin (I). 
and $d$ can produce eight different spin states $(2 \otimes 2 \otimes 2=4 \oplus 2 \oplus 2)$ : four spin-3/2 states that are completely symmetric, two spin- $1 / 2$ states that are symmetric in $u d$, and two spin- $1 / 2$ states that are anti-symmetric in $u d$. Therefore, the spins of the $\Lambda_{c}^{+}$quarks must be in an anti-symmetric spin$1 / 2$ state. The spins of the $\Sigma_{c}$ quarks can be in either a symmetric spin- $1 / 2$ state or a symmetric spin-3/2 state.

There are three $\Xi_{c}$ ground states: $\Xi_{c}$ with spin-parity $(1 / 2)^{+}, \Xi_{c}^{\prime}$ with spin-parity $(1 / 2)^{+}$, and $\Xi_{c}(2645)$ with spin-parity $(3 / 2)^{+}$. These three ground states are analogous to those just discussed for $\Lambda_{c}^{+}$and $\Sigma_{c}$. Considering $\mathrm{SU}(3)$ flavor symmetry between light quarks $u, d$, and $s$, the $\Xi_{c}$ states can be either flavor symmetric or flavor anti-symmetric. Again, the quark spins can make symmetric spin-3/2 states, symmetric spin-1/2 states, or anti-symmetric spin- $1 / 2$ states. Again, Fermi statistics requires that the combined spin-flavor wave function be symmetric under the interchange of the mass degenerate $u, d$, and $s$ quarks. Therefore, following the $\Lambda_{c}^{+}$and $\Sigma_{c}$ pattern, the $\Xi_{c}$ state is flavor anti-symmetric, and the $\Xi_{c}^{\prime}$ and $\Xi_{c}(2645)$ states are flavor symmetric.

The hadrons produced in the $c \bar{c}$ hadronization process are typically very short lived. Heavy and excited charm baryon states predominantly decay via the strong interaction and have lifetimes on the order of $10^{-23}$ seconds. Because of baryon number and energy conservation, these strong decays typically produce one lighter baryon and a small number of light mesons such as kaons or pions. When strong decays are not allowed, these states decay electromagnetically with typical lifetimes 1000 times longer than those of strongly decaying states. The arrows in Figure 1.2 represent the strong and electromagnetic decay modes of several of the charm-baryon states. Each decay arrow points from the original parent state to the daughter charm-baryon state the decay produces, and each is labeled by the daughter mesons (or photon) produced in the decay. When a charm baryon is in the lowest-mass configuration for its quark content, that baryon can only decay weakly. Such states in Figure 1.2 are the double-charm baryons and the lowest mass $\Omega_{c}, \Xi_{c}$, and $\Lambda_{c}$ states. All charged weak decays change the quark flavors that are present. Charm baryons that decay weakly have lifetimes on the order of $10^{-13}$ seconds. As an example, the $\Lambda_{c}^{+}$is the lowest-mass cud baryon and it decays weakly with a lifetime of $2 \times 10^{-13}$ seconds [7]. Among its larger branching fractions are $\mathcal{B}\left(\Lambda_{c}^{+} \rightarrow p K^{-} \pi^{+}\right)=(5.0 \pm 1.3) \%, \mathcal{B}\left(\Lambda_{c}^{+} \rightarrow p \overline{K^{0}}\right)=(2.3 \pm 0.6) \%, \mathcal{B}\left(\Lambda_{c}^{+} \rightarrow p \overline{K^{0}} \pi^{-} \pi^{+}\right)=(2.6 \pm 0.7) \%$, $\mathcal{B}\left(\Lambda_{c}^{+} \rightarrow \Lambda^{0} \pi^{+}\right)=(1.0 \pm 0.3) \%$, and $\mathcal{B}\left(\Lambda_{c}^{+} \rightarrow \Lambda^{0} \pi^{+} \pi^{-} \pi^{+}\right)=(2.6 \pm 0.7) \%$ [7]. Each of these $\Lambda_{c}^{+}$decay modes are used in my double-charm or charm-strange research. As can been seen from Figure 1.2, a large number of charm baryons have been observed or are predicted to produce $\Lambda_{c}^{+}$baryons in their decay. Reconstructing $\Lambda_{c}^{+}$baryons in the BABAR data is the starting point of my analysis.

\subsection{Double-Charm and Excited Charm-Strange Baryons}

The double-charm baryon and charm-strange baryon states that are the focus of my dissertation are shown indicated by the colored horizontal lines in Figure 1.2. The double-charm baryons (which are 
not verified with this study) are shown in blue, and the excited charm-strange states are shown in green. The two double-charm baryons are the isospin partners $\Xi_{c c}^{+}(c c d)$ and $\Xi_{c c}^{++}(c c u)$. The mass differences between members of all well established isospin multiplets are on the order of $1 \mathrm{MeV} / c^{2}$. The $60 \mathrm{MeV} / c^{2} M\left(\Xi_{c c}^{+}\right)-M\left(\Xi_{c c}^{++}\right)$mass difference shown in Figure 1.2 is from an unverified experimental result [2], which is discussed in more detail in Chapter 3. The charm-strange baryon pairs $\Xi_{c}(2970)^{+, 0}, \Xi_{c}(3055)^{+, 0}, \Xi_{c}(3077)^{+, 0}$, and $\Xi_{c}(3123)^{+, 0}$ are each isospin doublets. The only isospin doublet in this study for which both baryons are observed with statistically significant signals, $\Xi_{c}(3077)^{+, 0}$, has a measured mass difference between the two baryons of about $2 \mathrm{MeV} / c^{2}$. While the spin-parity of the ground-state $\Xi_{c c}^{+}$and $\Xi_{c c}^{++}$baryons are presumed, the orbital and radial excitations of the newly observed charm-strange baryons in this study are unknown. Recent theoretical work suggests some possible quantum numbers for the charm-strange baryons based on comparisons of masses, decay rates, and decay modes in experiment and theory [13, 14, 15, 16, 17].

The double-charm and charm-strange baryons studied here are short-lived particles that decay into longer-lived particles that are measured as they traverse through and interact with the BABAR Detector. The measurements of the longer-lived decay products are used to reconstruct the properties of the states from which they originate. I search for a $\Xi_{c c}^{+}$signal among reconstructed $\Lambda_{c}^{+} K^{-} \pi^{+}$ candidates. For the $\Xi_{c c}^{+}$, this is a weak decay with one of the charm quarks decaying into a strange quark. This weak decay is illustrated in Figure 1.3. The charm-strange baryons $\Xi_{c}(2970)^{+}$and $\Xi_{c}(3077)^{+}$were first observed [6] by the Belle Experiment in the same $\Lambda_{c}^{+} K^{-} \pi^{+}$final state. For the $\Xi_{c}(2970)^{+}$and $\Xi_{c}(3077)^{+}$, this is a strong decay in which QCD produces $u \bar{u}$ and $d \bar{d}$ quark anti-quark pairs; I illustrate this strong decay in Figure 1.4. The conservation of baryon number requires that the strong or weak decay of a baryon results in at least one newly formed baryon. For both the double-charm and the charm-strange baryons in my research, the one newly formed baryon is the $\Lambda_{c}^{+}$. I study several potential decay modes with a $\Lambda_{c}^{+}$, a charged or neutral kaon, and from zero to two charged pions. The research I present in this dissertation is wide ranging, but it is brought together through similar decay modes and similar measurements of QCD processes. 


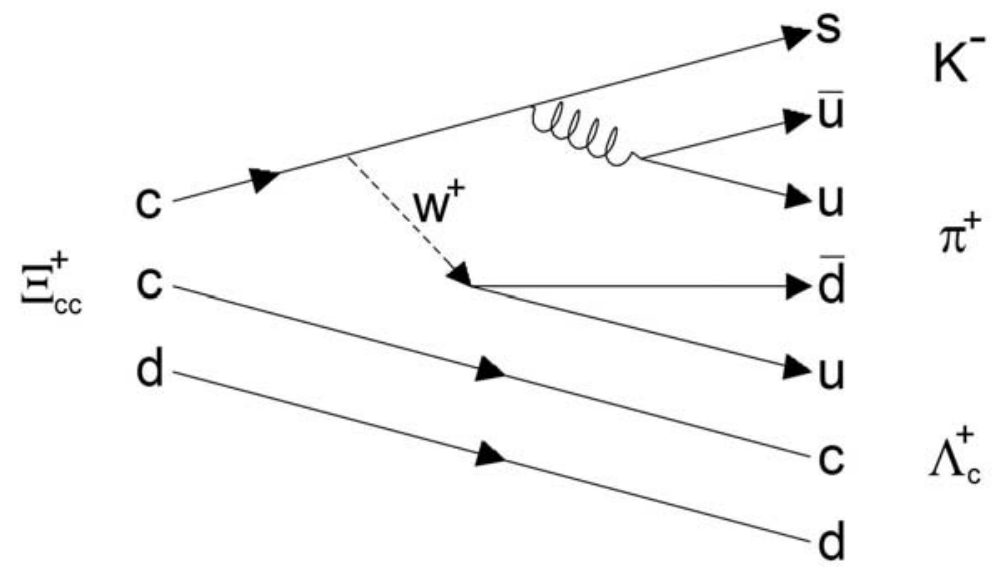

Figure 1.3: A Feynman diagram of the weak decay $\Xi_{c c}^{+} \rightarrow \Lambda_{c}^{+} K^{-} \pi^{+}$. The curled line represents a gluon, which produces a quark anti-quark pair in the decay process.

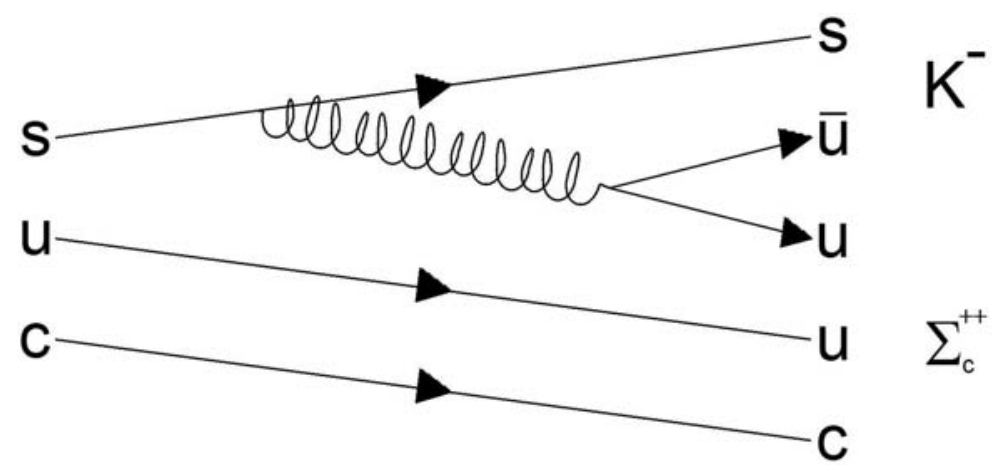

Figure 1.4: A Feynman diagram of a strongly decaying csu state. The curled line represents a gluon, which produces a quark anti-quark pair in the decay process. 


\section{Chapter 2}

\section{The BABAR Experiment}

All of my research described in this dissertation is carried out with the BABAR Detector, which was built and is operated by the BABAR Collaboration. This is a group of roughly 550 physicists from 10 countries and 77 institutions. The BABAR Collaboration works with the PEP-II storage rings to observe the results of high-energy $e^{+} e^{-}$events. The number of events created with PEP-II and recorded with the BABAR Detector are determined by the integrated luminosity, which has units of inverse cross-section. The BABAR Collaboration has integrated hundreds of inverse femtobarns of luminosity since operation began in mid-1999. Since the cross-sections for the types of recorded $e^{+} e^{-}$events are on the order of $1 \mathrm{nb}$ (see Table 1.1), roughly one billion events have been recorded. This large number of recorded events enables the BABAR Collaboration to perform highly sensitive studies and to make precise measurements. The primary studies of BABAR Collaboration are with $B$ mesons, which contain $b$ quarks from the decay of the $\Upsilon(4 S)$ resonance $(b \bar{b})$, but because of a large number of other types of fermion anti-fermion pairs produced in $e^{+} e^{-}$annihilations, many other physics studies can be carried out. This dissertation is an example of such an alternative study; my research is based on $e^{+} e^{-} \rightarrow \gamma^{*} \rightarrow c \bar{c}$ events recorded with the BABAR Detector.

The PEP-II storage rings are used to create head-on $e^{+} e^{-}$collision at a center-of-mass energy of $10.58 \mathrm{GeV}$. From the detector frame of reference, the electron and positron beams have unequal energies of $9.0 \mathrm{GeV}$ and $3.1 \mathrm{GeV}$, respectively. The reference frame of the $e^{+} e^{-}$center-of-mass has a Lorentz boost, with respect to the detector, of $\beta \gamma=0.56$ in the direction of the electron beam. This boost causes the short-lived products of the $e^{+} e^{-}$events, such as $B$ mesons, to travel distances measurable with the BABAR Detector. The BABAR Detector is designed to have a forward-backward asymmetry along the beam axis to accommodate the boosted $e^{+} e^{-}$events; detector subsystems are grouped toward the front end (electron beam direction) of the detector while support systems are grouped toward the back end (positron beam direction).

The beam-energy and detector asymmetries are designed to enable the study of $B$-meson mixing and indirect charge-parity $(C P)$ violation. The center-of-mass energy of $10.58 \mathrm{GeV}$ is the mass of the 
$\Upsilon(4 S)$ resonance. Colliding the $e^{+} e^{-}$beams at this energy maximizes the number of $\Upsilon(4 S)$ mesons produced. The $\Upsilon(4 S)$ is a $b \bar{b}$ resonance that predominantly decays to a pair of $B$ mesons. Because of the $B \bar{B}$ center-of-mass boost, the lifetime of the $B$-mesons are measurable by the hundreds of microns that the $B$ mesons travel before they decay. When neutral $B$-mesons decay to a $C P$ eigenstate, interference between the amplitudes of the decay with and without mixing manifests as a distance-dependent (proper-time dependent) decay rate; this phenomenon is called indirect $C P$ violation. To study this indirect $C P$ violation is the primary intent of the BABAR Collaboration.

Besides $B$-meson mixing and indirect $C P$ violation, there are many other physics studies that can and are carried out with the BABAR Detector. Other types of $e^{+} e^{-}$events are recorded with the BABAR Detector and many discoveries and measurements have been made with these data. Our large amount of integrated luminosity gives us unprecedented sensitivity to Standard Model physics as well as physics beyond the Standard Model. Hundreds of results from the BABAR Collaboration have been published in scientific journals. I use the large amount of data to look for new particles produced in the $c \bar{c}$-hadronization process and to make precise measurements of particle mass and width (which is related to particle lifetime and decay rate). Reference [4] reports some of my research with the BABAR Collaboration on double-charm baryons.

\subsection{The BABAR Detector}

The BABAR Detector is designed to electronically record precise $e^{+} e^{-}$event information at rates in excess of $100 \mathrm{~Hz}$. Figures 2.1 and 2.2 show two views of the BABAR Detector; it is described in detail in Reference [10]. The detector is divided into five subsystems. Each subsystem is designed to measure particular aspects of the particles produced from the PEP-II $e^{+} e^{-}$events. The tracking of charged particles is provided by a five-layer double-sided silicon vertex tracker (SVT) and a 40-layer drift chamber (DCH). Discrimination between charged pions, kaons, and protons relies on ionization energy loss $(\mathrm{d} E / \mathrm{d} x)$ in the $\mathrm{DCH}$ and SVT, and on Cherenkov photons detected in a ring-imaging detector (DIRC). A CsI(Tl) crystal electromagnetic calorimeter (EMC) is used to identify electrons and photons. These four detector subsystems are mounted inside a 1.5-T solenoidal superconducting magnet that creates a magnetic field parallel to the z-axis of the detector as shown in Figures 2.1 and 2.2. The instrumented flux return (IFR) for the solenoidal magnet provides muon identification. A hardware trigger system based on DCH and EMC information passes time-windowed data out of all five subsystems at a rate of about 2500 events per second. Software-based algorithms are used to reduce by a factor of about 20 the number of events that are further processed and stored. The detector subsystems most relevant to this dissertation are the SVT, the DCH, and the DIRC; each are described in more detail below. 


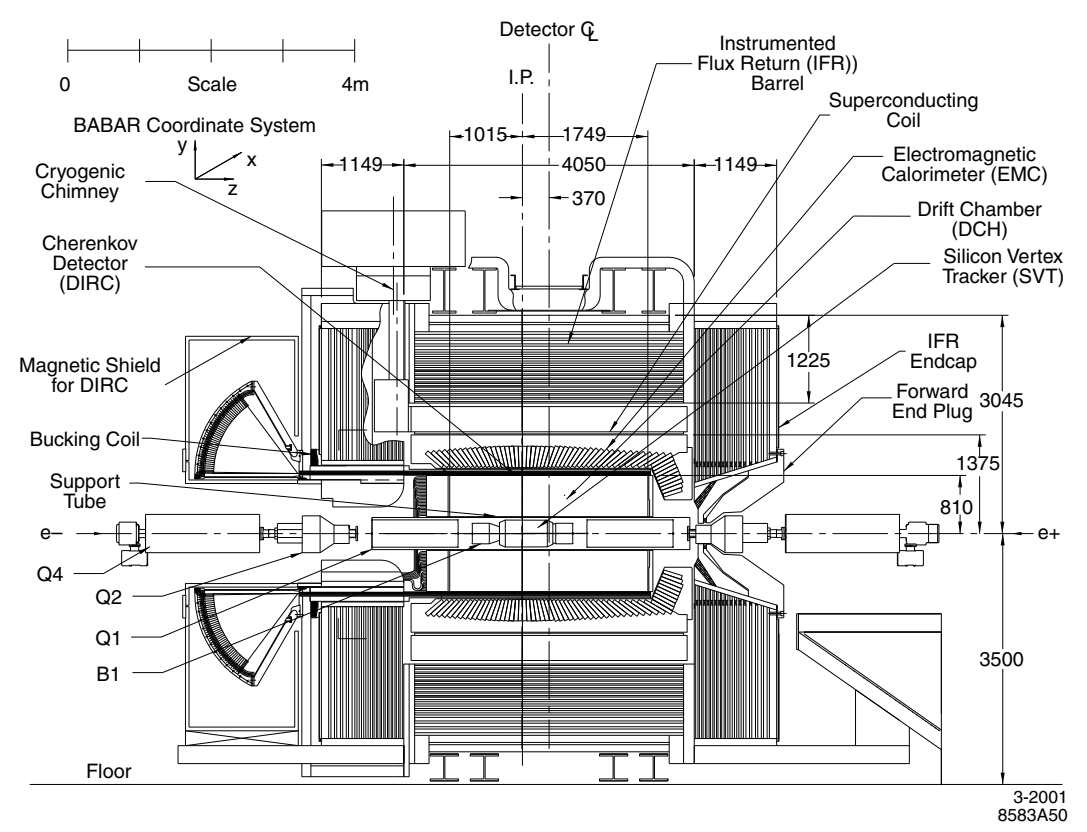

Figure 2.1: Longitudinal section through the BABAR Detector. The drawing is to scale and has major subsystems and components labeled. The $9.0 \mathrm{GeV}$ electrons enter from the left (backward side) and the $3.1 \mathrm{GeV}$ positrons from the right (forward side). B1, Q1, Q2, and Q4 are PEP-II dipole and quadrupole magnets.

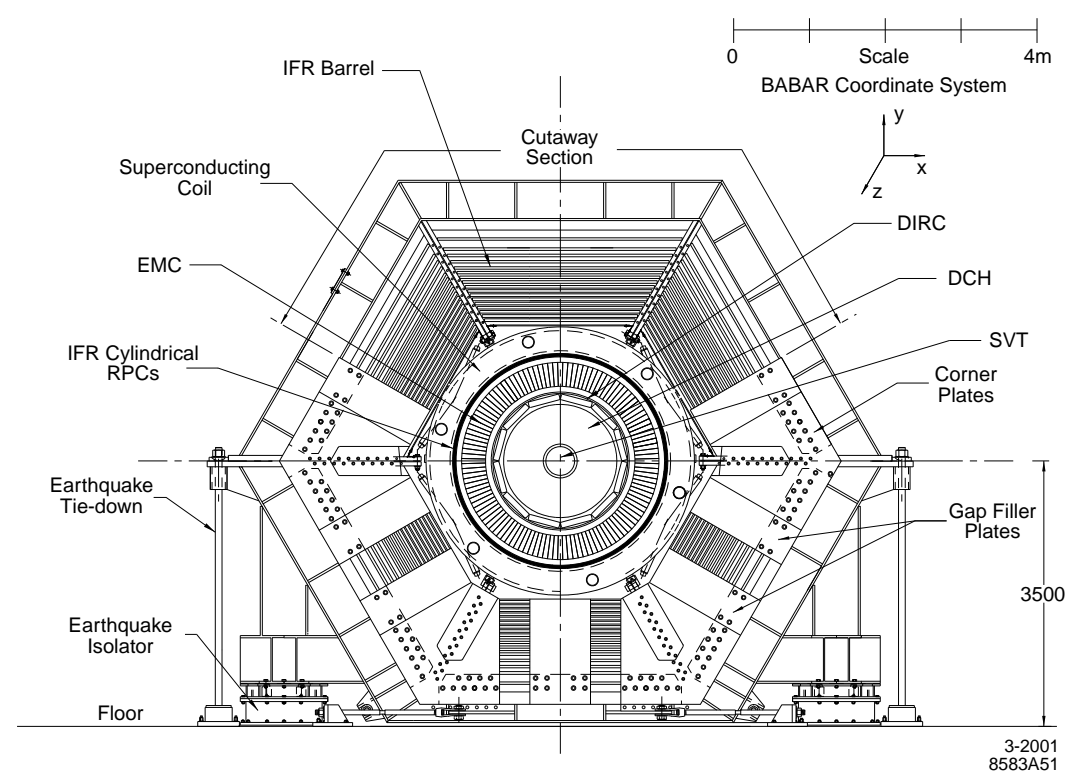

Figure 2.2: End view of the BABAR Detector. The drawing is to scale and has major subsystems and components labeled. 


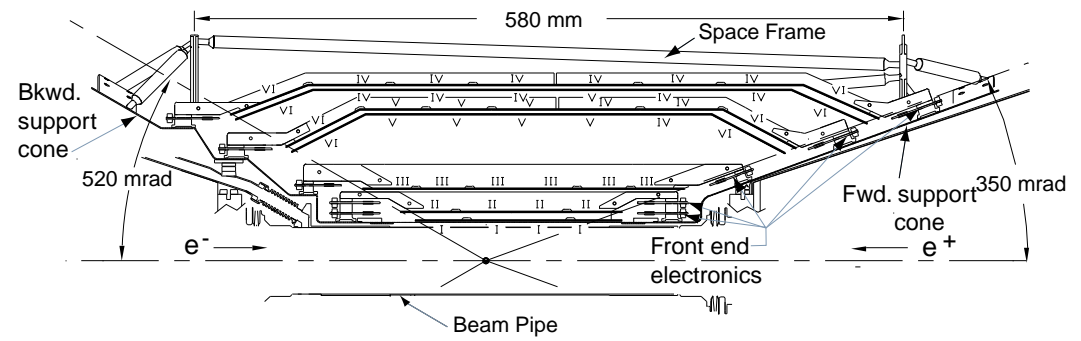

Figure 2.3: Longitudinal section through the SVT. The SVT is located forward of the $e^{+} e^{-}$interaction point in order to compensate for the boosted $e^{+} e^{-}$center of mass.

\subsubsection{The Silicon Vertex Tracker}

The SVT [18] is the innermost subsystem of the BABAR Detector. Figure 2.3 shows a longitudinal section through the SVT. It consists of 52 modules of double-sided silicon strip sensors arranged into five layers that surround the $e^{+} e^{-}$interaction point. The strip spacing varies from about $50 \mu \mathrm{m}$ to about $210 \mu \mathrm{m}$. The strips on one side of each module run parallel to the beam axis and the strips on the opposite side run transverse to the beam axis. There are about 150,000 strips in total. The strips are monitored with charge-sensitive integrated circuits with circular buffers that pass on their information upon a signal from the trigger system. The innermost layer of the SVT is just $3.2 \mathrm{~cm}$ from the $e^{+} e^{-}$interaction point. The modules are strategically placed towards the forward direction to compensate for the boosted $e^{+} e^{-}$center of mass. In the detector frame, the SVT has a forward acceptance angle of $350 \mathrm{mrad}$ from the beam line and a backward acceptance angle of $520 \mathrm{mrad}$. The five-layer design of the SVT provides precision for determining the trajectory of charged particles produced from the $e^{+} e^{-}$events, independent of the $\mathrm{DCH}$. It also helps to provide $\mathrm{d} E / \mathrm{d} x$ information that is used to identify particles.

\subsubsection{The Drift Chamber}

The DCH subsystem [19] surrounds the SVT to provide additional tracking of charged particles (which primarily determines transverse momentum) and to measure $\mathrm{d} E / \mathrm{d} x$. It is also essential in determining the decay vertexes of longer-lived neutral particles, such as the $\Lambda^{0}$ and the $K_{s}$, that can decay into charged particles many centimeters away from the $e^{+} e^{-}$interaction point. The momenta, transverse to the z-axis, of charged particles are derived from the curvature of the reconstructed particle trajectories. These trajectories are curved because of the 1.5-T magnetic field parallel to the z-axis of the detector. A longitudinal section through of the DCH is shown in Figure 2.4. The $\mathrm{DCH}$ is $275 \mathrm{~cm}$ in length and extends from an inner radius of $22.5 \mathrm{~cm}$ to an outer radius of $80.0 \mathrm{~cm}$ near the DIRC. The DCH consists of hexagonal cells of wires arranged into 40 layers, in a 80:20 


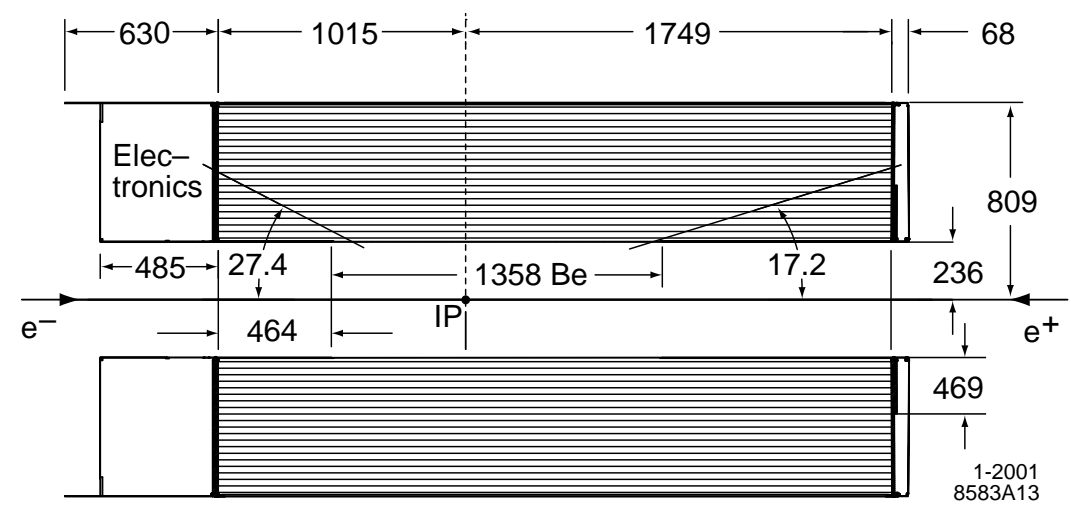

Figure 2.4: Longitudinal section through the DCH. The interaction point (IP) of the electron and positron beams is off-center to accommodate the boosted $e^{+} e^{-}$center of mass. The DCH electronics are placed on the backward side where less detector coverage is needed. Distances are in millimeters and angles are in degrees.

mixture of helium:isobutane. Of the 40 layers, 24 have wires that are at small angles to the z-axis so that longitudinal information can be derived. In total, there are 7,104 wires that provide DCH measurements. DCH material is kept to a minimum to reduce the scattering of tracked particles, which would degrade the measurements of track parameters. The forward acceptance angle of the DCH is $300 \mathrm{mrad}$ and the backward acceptance angle is $480 \mathrm{mrad}$. The DCH electronics are placed on the backward side where less detector coverage is needed.

The combination of DCH and SVT information provides for excellent charged particle tracking. A $1.0 \mathrm{GeV} / c$ track's point of closest approach to the $e^{+} e^{-}$interaction point has resolutions parallel and transverse to the z-axis of $65 \mu \mathrm{m}$ and $55 \mu \mathrm{m}$, respectively; the resolution for azimuthal angle about to the $\mathrm{z}$-axis is $1.0 \mathrm{mrad}$ and the resolution for the tangent of the polar angle is 0.001 . The resolution for measured transverse momentum is $0.5 \%$ and the $\mathrm{d} E / \mathrm{d} x$ resolution is $7.5 \%$ for particles with $1.0 \mathrm{GeV} / c$ momentum. Tracks are categorized into one of four levels of tracking quality: ChargedTracks, VeryLoose, Loose, and Tight. Each successive level has more stringent requirements. Any identified track passes the ChargedTracks level. The VeryLoose level requires a track to pass within $1.5 \mathrm{~cm}$ of the beam line, and this point of closest approach must be within $10 \mathrm{~cm}$ (along the z-axis) of the $e^{+} e^{-}$ interaction point. In addition to these requirements, the Loose level requires a track to indicate that the charged particles transverse momentum is greater than $100 \mathrm{MeV} / c$ and that there are at least 12 points of information measured with the DCH. The Tight level has the more stringent requirements of 20 points of information from the $\mathrm{DCH}$, a track must pass within $1.0 \mathrm{~cm}$ of the beam line, and this point of closest approach must be within $3 \mathrm{~cm}$ of the $e^{+} e^{-}$interaction point. 


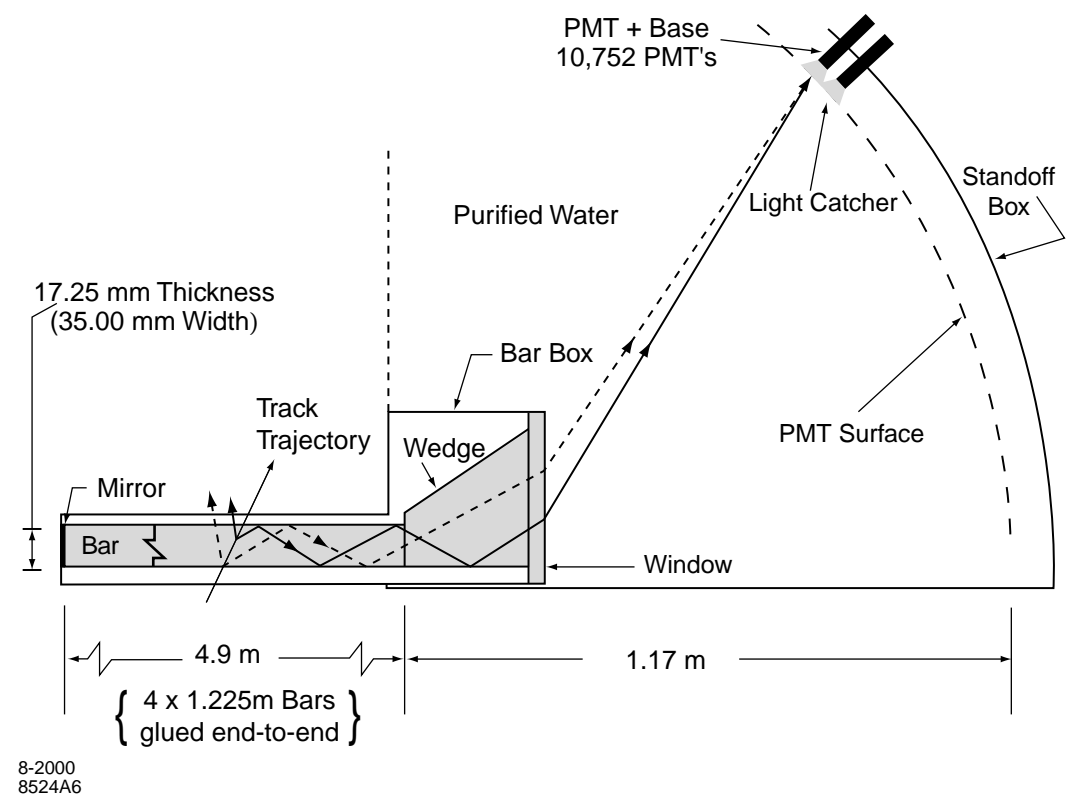

Figure 2.5: Longitudinal section through the DIRC subsystem showing a fused silica radiator bar and the imaging system.

\subsubsection{The Cherenkov Ring-Imaging Detector}

The DIRC subsystem [20] is used to identify charged hadrons. This is achieved through the use of Cherenkov radiation from relativistic charged hadrons that pass through fused-silica radiator bars. The DIRC radiator material is in the form of long bars of thin rectangular cross-section; the bars are nominally $17.25 \mathrm{~mm}$ thick (in the radial direction), $35 \mathrm{~mm}$ wide (azimuthally), and $4.9 \mathrm{~m}$ long. These bars are arranged into dodecagon-barrel shape that surrounds the $\mathrm{DCH}$. The Cherenkov radiation is emitted at an angle $\theta_{C}=\arccos (1 / n \beta)$ with respect to the hadron flight path, where $n$ is the index of refraction of the fused silica and $\beta$ is the speed of the hadron relative to the speed of light in vacuum. A Cherenkov photon is internally reflected within a radiator bar until it exits the backward end of the bar (possibly after reflecting off a mirror on the frontward end of the bar) and enters a water-filled expansion region. 10,752 photomultiplier tubes covering the expansion region are used to detect the distorted conic sections of Cherenkov radiation from each charged particle. The measured parameters of the conic sections are related back to the Cherenkov angle. Figure 2.5 shows a schematic of a DIRC radiator bar and imaging system.

\subsection{Data Acquisition and Triggering}

The BABAR Detector operates with a trigger system for the read out of detector electronics and the recording of data. There is a hardware trigger that passes event information at about $2.5 \mathrm{kHz}$ 
into a software trigger that passes event information on to storage at about $120 \mathrm{~Hz}$; both levels of triggering decisions are based on DCH and EMC information. The hardware trigger receives data continuously from the DCH and EMC. It processes this information with a fast tracking algorithm that counts the number of tracks in the $\mathrm{DCH}$ and determines their approximate direction. The hardware trigger is passed under any of three consitions: if there are a large number of tracks, the tracks roughly match the direction of clusters of energy detected in the EMC, or there is a large amount of energy measured in the EMC. The software trigger uses additional detector information to better reconstruct events. The combined efficiency for the hardware and software triggers to pass desired $e^{+} e^{-}$physics events varies from about $92 \%$ for $e^{+} e^{-} \rightarrow \gamma \rightarrow \tau^{+} \tau^{-}$to greater than $99 \%$ for $e^{+} e^{-} \rightarrow \gamma \rightarrow b \bar{b}$. The combined trigger efficiency for $e^{+} e^{-} \rightarrow \gamma \rightarrow c \bar{c}$ is about $99 \%$.

\subsection{Particle Identification}

The ionization energy loss $(\mathrm{d} E / \mathrm{d} x)$ of a charged particle moving through matter is related to the momentum and mass of that particle through the Bethe-Bloch equation [7]. Particle momenta transverse to the z-axis of the BABAR Detector are determined from the curvature of their tracks in the 1.5-T magnetic field; the polar angle of a track is used to determine its longitudinal momentum. The amount of electric charge measured with the DCH wires and the SVT strips is proportional to a particles energy loss. With these pieces of information, charged particles of different mass can be discriminated. Figure 2.6 shows the measured $\mathrm{d} E / \mathrm{d} x$ versus momentum as determined with the DCH. The curves in Figure 2.6 represent the Bethe-Bloch equation for the electron, muon, pion, kaon, proton, and deuteron hypotheses. The data lie near the Bethe-Bloch curves. For each reconstructed track, particle identification (PID) likelihoods are calculated for each mass hypothesis based on the measured $\mathrm{d} E / \mathrm{d} x$ and momentum, and the Bethe-Bloch equation for that particle mass.

The DCH can only satisfactorily distinguish $\pi$ and $K$ with lab momenta up to about $700 \mathrm{MeV} / c$. The DIRC provides particle discrimination for higher momentum particles; it is designed to perform in the momentum range $700 \mathrm{MeV} / c$ to $4200 \mathrm{MeV} / c$. PID likelihoods for the DIRC are based on the particle momentum, Cherenkov angle, number of DIRC photons, and tracking quality. Figure 2.7 shows the measured Cherenkov angle versus momentum for charged pions. The curves in Figure 2.7 represent the expected Cherenkov angle versus momentum for different particle-type hypotheses. The measured data lie near the curves.

For identifying particle types, the BABAR Collaboration often uses a system of likelihood ratios based on the combined PID likelihoods from the SVT, DCH, and DIRC information. The level of discrimination is categorized into one of four levels: VeryLoose, Loose, Tight, and VeryTight. Each successive level has more stringent requirements on the likelihood ratios between the various mass hypotheses. As an example, for a VeryLoose identification of a kaon, the ratio of kaon to pion likelihood has to be greater that 0.5 ; for a Tight identification of a kaon, the ratio of kaon to pion 


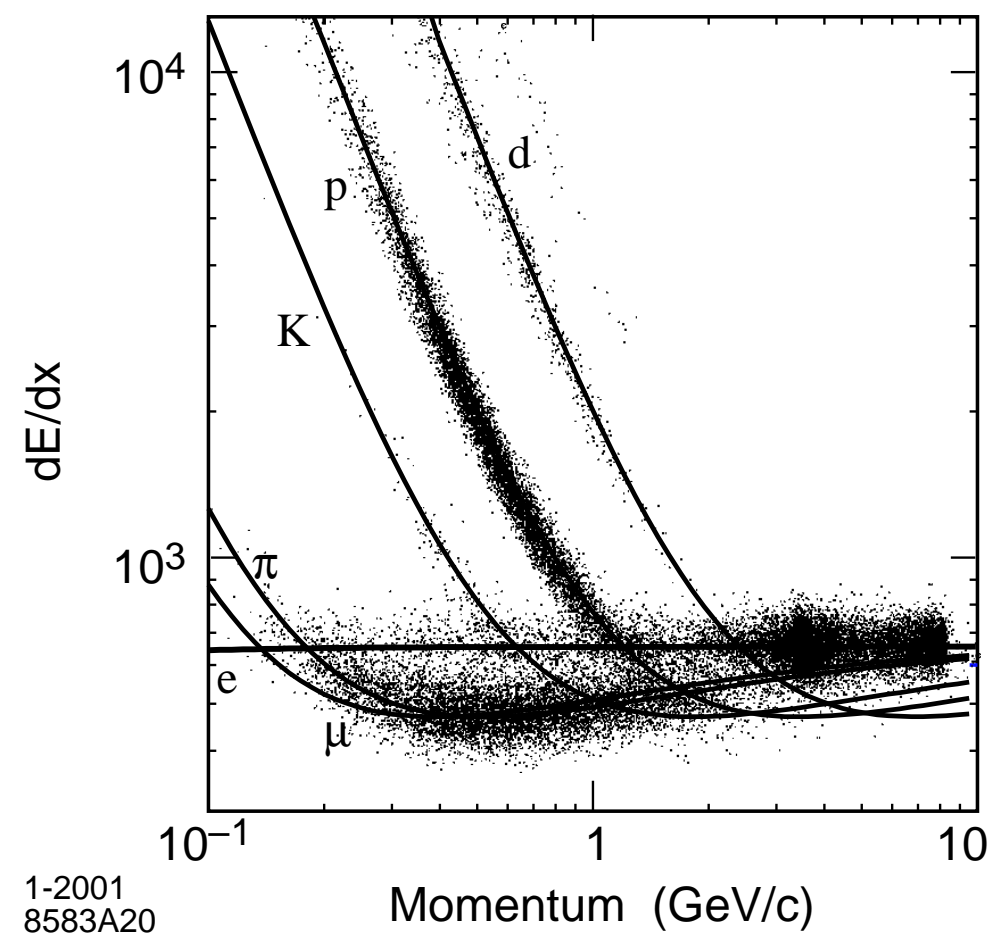

Figure 2.6: Measured $\mathrm{d} E / \mathrm{d} x$ (arbitrary units) versus momentum as determined with the DCH. The curves represent the Bethe-Bloch equation for different mass hypotheses.

likelihood has to be greater that 0.9 . The more stringent requirements increase the purity of the sample of particles that pass, but reduce the efficiency.

\subsection{BABAR Data Analysis}

The BABAR Collaboration has developed a collection of software tools to facilitate our common data analysis needs. This data analysis software is frequently updated for better performance, and extensive SLAC computing resources are used to run the BABAR data processing applications. The data recorded by $B A B A R$ Collaboration takes up roughly one petabyte of storage. This sample of all recorded events is processed in order to provide smaller subsets with particular types of events that are used in different data analyses. Often, data analyses performed by BABAR collaborators are done in teams. I did not work with a team to perform the analyses that I describe in this dissertation, but I did have the guidance of many collaborators and relied on many analysis resources developed by the BABAR Collaboration.

$B A B A R$ event reconstruction software is used to extract physics information from the raw recorded data. Tracking software uses information from the SVT and DCH to calculate trajectories and momenta of charged particles. When looking for particles that decay and create two or more charged 


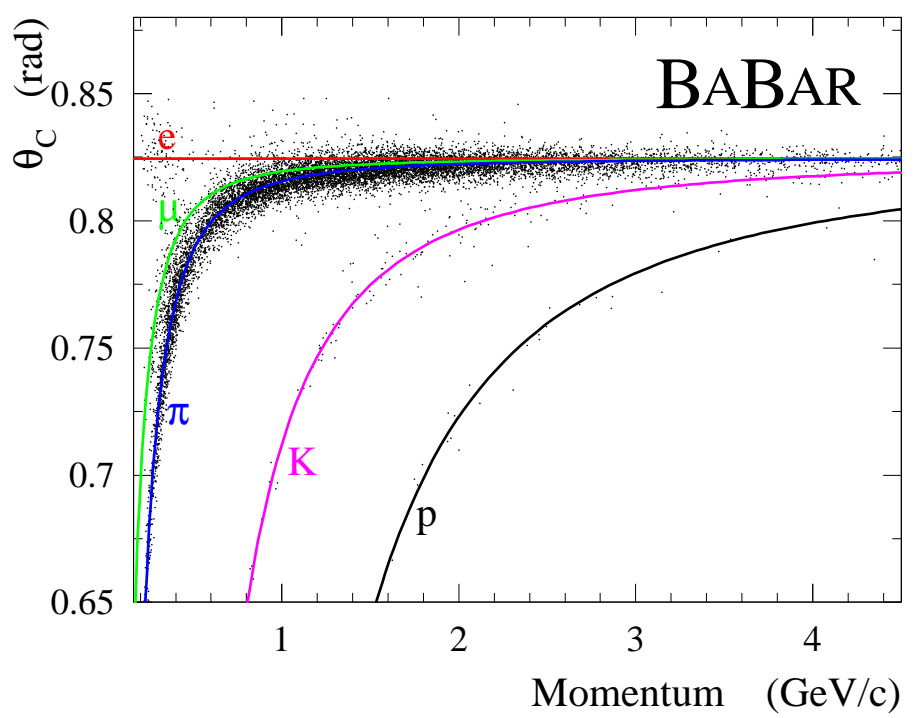

Figure 2.7: Measured Cherenkov angle versus momentum for charged pion candidates. The curves represent the expected Cherenkov angle versus momentum for different particle-type hypotheses.

particles, all possible track combinations in an $e^{+} e^{-}$event can be analyzed to determine how likely it is that the tracks originate from the same point. A fit of the tracks is performed using the geometric constraint that all considered track trajectories intersect at the same point in space. The $\chi^{2}$ probability of this fit is used to determine the validity of the hypothesis that the tracks originate from the same point. Often, particles produced in an $e^{+} e^{-}$event have a sequence of decays before the final charged particles that traverse through the detector are produced. In my analysis of doublecharm baryons, each decay vertex has a separate geometrically constrained fit. In my analysis of charm-strange baryons, all the reconstructed tracks in a proposed decay tree are fit simultaneously using multiple decay vertexes that are each geometrically constrained.

When tracks are constrained to a common vertex to form a candidate for a parent particle, that parent candidate's four-momentum is calculated from the fitted four-momenta of the daughter tracks. The parent's four-momentum yields the invariant mass of the parent particle. For a sample of parent candidates that are accurately reconstructed from true daughter tracks, the invariant-mass distribution normally peaks at the mass corresponding to the parent particle type. The natural width of this invariant-mass distribution is directly related to the mean lifetime of the decaying particle. For particles with relatively long lifetimes, this natural width is too narrow to be resolved with the BABAR Detector. Typical invariant-mass experimental resolutions for the BABAR Detector are a couple of $\mathrm{MeV} / \mathrm{c}^{2}$; a natural width of a couple $\mathrm{MeV} / \mathrm{c}^{2}$ would correspond to a mean particle lifetime of about $5 \times 10^{-22}$ seconds. 
Often, a parent candidate is reconstructed from one or more daughter candidates that are also parent candidates themselves. When there is a tree of decays such as this, the invariant-mass resolution for the original parent is degraded. My double-charm baryon analysis and my charm-strange baryon analysis use different techniques to improve the invariant-mass resolution of the original parent. For the double-charm baryon analysis, the original parent is the double-charm baryon candidate. One of the reconstructed double-charm baryon daughters is a $\Lambda_{c}^{+}$baryon candidate. The $\Lambda_{c}^{+}$baryon is also the parent candidate for a combination of $p, K^{-}$, and $\pi^{+}$. Instead of simply analyzing the double-charm baryon candidate invariant-mass distributions, I analyze distributions of the difference between the invariant mass of the double-charm baryon candidate and that of its $\Lambda_{c}^{+}$daughter candidate. The reconstructed mass of the double-charm baryon candidate and its $\Lambda_{c}^{+}$ daughter candidate share correlated errors, and using their mass difference removes these correlated errors. When analyzing charm-strange baryons, I use a different technique with a similar result; I perform mass-constrained fits where the invariant-mass of a daughter candidate is fixed to a predetermined mass value. Doing so improves the invariant-mass resolution of the parent by improving the measurement of the momentum of the daughter candidate.

In order to estimate invariant-mass resolutions and reconstruction efficiencies, the BABAR Collaboration uses $e^{+} e^{-}$event and detector simulations generally referred to as Monte Carlo (MC) simulations. For these event simulations, we use the MC generators JETSET74 [21] and EVTGEN [22] with a full detector simulation based on GEANT4 [23]. The production and particle decay trees can be specified in these simulations in order to simulate events with signal candidates for a particular analysis. I use such signal-MC samples to estimate invariant-mass resolutions and reconstruction efficiencies when analyzing double-charm baryon and charm-strange baryon candidates in data. Background samples are also simulated by creating generic types of $e^{+} e^{-}$events that do not involve any signal processes. Analyzing background MC samples helps to estimate both the amount of background contributing to a data sample and the shapes of background distributions. 


\section{Chapter 3}

\section{Double-Charm Baryons}

In this chapter I describe my search for the $e^{+} e^{-}$production of $\Xi_{c c}^{+}$and $\Xi_{c c}^{++}$baryons in their weak decays to $\Lambda_{c}^{+} K^{-} \pi^{+}$and $\Lambda_{c}^{+} K^{-} \pi^{+} \pi^{+}$, respectively, where $\Lambda_{c}^{+} \rightarrow p K^{-} \pi^{+}$. The $\Xi_{c c}^{+}$and $\Xi_{c c}^{++}$baryons are the two ground states predicted for baryons with two charm quarks. They have isospin $I=1 / 2$, orbital angular momentum $L=0$, and total angular momentum $J=1 / 2$.

Theoretically these double-charm baryons could have a heavy-diquark light-quark substructure $[24,25,26]$ with the two charm quarks forming the heavy diquark and the up or down quark being, in comparison, light. It has been proposed that the $c c$ diquark could be in one of two different spin states [26]. With an orbital angular momentum of $L=0$, the two spin- $1 / 2$ charm quarks may combine in a spin- 1 triplet or a spin-0 singlet. The two identical charm quarks must be in a state that is antisymmetric under their interchange. If the diquark has spin 1 then the spin part of the wave function for the baryon is symmetric under the interchange of the two charm quarks. If the diquark has spin 0 , then the spin part of the wave function for the baryon is anti-symmetric under the interchange. For the symmetric spin state, the total wave function is anti-symmetrized by the anti-symmetric color-singlet state of the baryon. The anti-symmetric spin state, however, requires a symmetric color-singlet state for the baryon. This latter case can be achieved via the inclusion of a gluon into the bound state of a baryon. It is possible that both of these types of double-charm baryon states are produced at similar rates and can be found at similar masses [26].

Various theoretical calculations predict $\Xi_{c c}^{+}$and $\Xi_{c c}^{++}$masses anywhere between $3.478 \mathrm{GeV} / c^{2}$ and $3.808 \mathrm{GeV} / c^{2}[27,28,29,30,31]$. The theory of QCD does not lend itself to calculating baryon masses, and the above references use models of QCD, quarks, and diquark for their predictions. The mass splitting between the $\Xi_{c c}^{+}$and the $\Xi_{c c}^{++}$is theoretically predicted to be on the order of $1 \mathrm{MeV} / c^{2}$. The lifetimes of $\Xi_{c c}^{+}$and $\Xi_{c c}^{++}$are expected to be between about $0.1 \mathrm{ps}$ and $1.0 \mathrm{ps}[11,12]$. The charmed baryons $\Lambda_{c}^{+}$and $\Xi_{c}^{+}$have measured lifetimes of $0.2 \mathrm{ps}$ and $0.4 \mathrm{ps}$, respectively [7].

The discovery of double-charm baryons would be a boon to the study of QCD. The validity of using diquarks in theoretical models could be tested with such experimental results. Both the 
Table 3.1: Theoretical predictions for double-charm baryon and $c c$ diquark production cross-sections in $e^{+} e^{-}$annihilations with about $10.58 \mathrm{GeV}$ center-of-mass energy. Approximate predicted numbers of double-charm baryons, assuming $232 \mathrm{fb}^{-1}$ of integrated luminosity, are also listed.

\begin{tabular}{ccc}
\hline Reference & Cross-Section Prediction & Number \\
\hline \hline$[24]$ & $\sigma\left(X_{c c}\right)=(70 \pm 10) \mathrm{fb}$ & 16,000 \\
\hline$[25]$ & $\sigma(c c-$ diquark $)=1 \mathrm{fb}$ & 232 \\
\hline$[26]$ & $\sigma\left(\Xi_{c c}\right)=230 \mathrm{fb}$ & 53,000 \\
\hline
\end{tabular}

interaction of the two heavy quarks within the diquark and the interaction of the heavy diquark with a light third quark are accessible through the study of double-charm baryons. The internal diquark interaction should also be comparable to quarkonium states while the diquark and light third quark interaction should be comparable to $Q \bar{q}$ mesons (where the $Q$ quark is heavy relative to the $q$ quark). Diquark models are currently understood through non-relativistic QCD where the relative velocities of the two heavy quarks, $v_{Q} / c$, is small enough to allow for perturbative expansions in $v_{Q}$. The QCD interaction between the diquark and the light third quark must be studied non-perturbatively.

For $\Xi_{c c}^{+}$and $\Xi_{c c}^{++}$, the relatively large branching fractions of $\mathcal{B}\left(\Xi_{c c}^{+} \rightarrow \Lambda_{c}^{+} K^{-} \pi^{+}\right)=0.03$ and $\mathcal{B}\left(\Xi_{c c}^{++} \rightarrow \Lambda_{c}^{+} K^{-} \pi^{+} \pi^{-}\right)=0.05$ have been predicted [32]. Several theoretical predictions, listed in Table 3.1, have also been made for the production cross-sections of $c c$ diquarks and double-charm baryons in $e^{+} e^{-}$collisions $[24,25,26]$. These predictions translate into anywhere from hundreds to tens of thousands of double-charm baryons being recorded in the BABAR data set. Theoretical cross-section predictions for double $c \bar{c}$ production have been found to be an order of magnitude too low. For example, the rate of $e^{+} e^{-} \rightarrow \gamma \rightarrow J / \psi \eta_{c}$ events in BABAR has been measured to be $7.6 \pm 1.9$ times that predicted by non-relativistic QCD [33]. These theoretical $c \bar{c}$ and $c c$ cross-section calculations are very similar and suggest that the predicted double-charm baryon production rates may also be underestimated.

Observations of both $\Xi_{c c}^{+}$and $\Xi_{c c}^{++}$have been reported by the SELEX Collaboration $[1,2,3]$. The SELEX Collaboration runs a charm hadroproduction experiment (E781) that uses a hyperon beam at the Fermi National Accelerator Laboratory (Fermilab). Evidence for $\Xi_{c c}^{+} \rightarrow \Lambda_{c}^{+} K^{-} \pi^{+}$is claimed at an invariant-mass of about $3.52 \mathrm{GeV} / c^{2}$ with 22 observed events including $6.1 \pm 0.5$ expected background events. SELEX also reports an observation of this $\Xi_{c c}^{+}$state decaying to $p D^{+} K^{-}$with 7 observed events including 1.6 expected background events. Evidence for $\Xi_{c c}^{++} \rightarrow \Lambda_{c}^{+} K^{-} \pi^{+} \pi^{+}$ is claimed at an invariant-mass of about $3.46 \mathrm{GeV} / c^{2}$ with 9 observed events including 1 expected background event.

There has been no verification of the SELEX double-charm baryon results. The SELEX evidence for $\Xi_{c c}^{+}$and $\Xi_{c c}^{++}$implies dynamics for the double-charm baryons that are unprecedented. One oddity 
is the $60 \mathrm{MeV} / c^{2}$ mass difference between $\Xi_{c c}^{+}$and $\Xi_{c c}^{++}$when the only theoretical difference between the two states is a $d$ versus a $u$ quark. All other isospin partner states previously observed have mass differences an order of magnitude smaller. Another oddity is the high production cross-section implied, where over $1 \%$ of all $\Lambda_{c}^{+}$baryons produced with the SELEX experiment are from decays of double-charm baryons [34].

The FOCUS Collaboration (also at Fermilab) operates a photoproduction experiment. They have also performed a search for these double-charm baryons but with negative results [5]. Their search folded 19,000 $\Lambda_{c}^{+}$baryons and 1,000,000 D mesons into 18 different double-charm baryon decay modes. The FOCUS result implies that, for the SELEX results to be correct, there needs to be an unusual and unexplained hadroproduction mechanism that is unique to hyperon beams.

\subsection{The Search Strategy}

I search for the $e^{+} e^{-}$production of double-charm baryon states that decay to $\Lambda_{c}^{+} K^{-} \pi^{+}$or $\Lambda_{c}^{+} K^{-} \pi^{+} \pi^{+}$, where $\Lambda_{c}^{+} \rightarrow p K^{-} \pi^{+}$. Signals for such states would indicate double-charm baryons by matching theoretical masses and decay modes. Also, the natural width (lifetime) of the signals would need to be consistent with a weakly decaying particle; weakly decaying particles have natural widths much narrower than our resolution with the BABAR Detector. The double-charm baryon decay modes that I utilize in my search have been predicted to have relatively large branching fractions [32], and they are decay modes in which the SELEX Collaboration has reported finding evidence for double-charm baryons.

I study the reconstructed invariant-mass differences $M\left(\left(p K^{-} \pi^{+}\right) K^{-} \pi^{+}\right)-M\left(p K^{-} \pi^{+}\right)$and $M\left(\left(p K^{-} \pi^{+}\right) K^{-} \pi^{+}\right)-M\left(p K^{-} \pi^{+}\right)$along with the $\Lambda_{c}^{+}$invariant mass $M\left(p K^{-} \pi^{-}\right)$. The $p K^{-} \pi^{+}$ are grouped together to indicate that they are used to form $\Lambda_{c}^{+}$candidates. Using the difference between the mass of the fully reconstructed signal candidate and that of the reconstructed $\Lambda_{c}^{+}$candidate improves the invariant-mass resolution. Including the $\Lambda_{c}^{+}$mass as a second dimension in the study improves the search sensitivity. The analysis of the BABAR data is performed in a "blind" fashion, where the invariant-mass distributions in the region where the signals potentially lie are not studied until data selection criteria and statistical procedures have been finalized. Both $\Xi_{c c}^{+}$ and $\Xi_{c c}^{++}$baryons are searched for in a mass-difference range of $1100 \mathrm{MeV} / c^{2}$ to $1310 \mathrm{MeV} / c^{2}$; this range is about 100 times wider than the mass-difference resolution. The search range corresponds to a double-charm baryon mass range from approximately $3390 \mathrm{MeV} / c^{2}$ to $3600 \mathrm{MeV} / c^{2}$. The $\Xi_{c c}^{+}$ and $\Xi_{c c}^{++}$baryons are also searched for specifically at the masses reported by SELEX: $3519 \mathrm{MeV} / c^{2}$ and $3460 \mathrm{MeV} / \mathrm{c}^{2}$, respectively. Figure 3.1 shows theoretically predicted and experimentally reported $\Xi_{c c}^{+}$and $\Xi_{c c}^{++}$masses with respect to the blinded mass-difference search region. The search region is roughly centered on the SELEX observations and includes two theoretically predicted masses as well. The $M\left(p K^{-} \pi^{+}\right)$range that I use is from $2256 \mathrm{MeV} / c^{2}$ to $2316 \mathrm{MeV} / c^{2}$ and surrounds the $\Lambda_{c}^{+}$ 


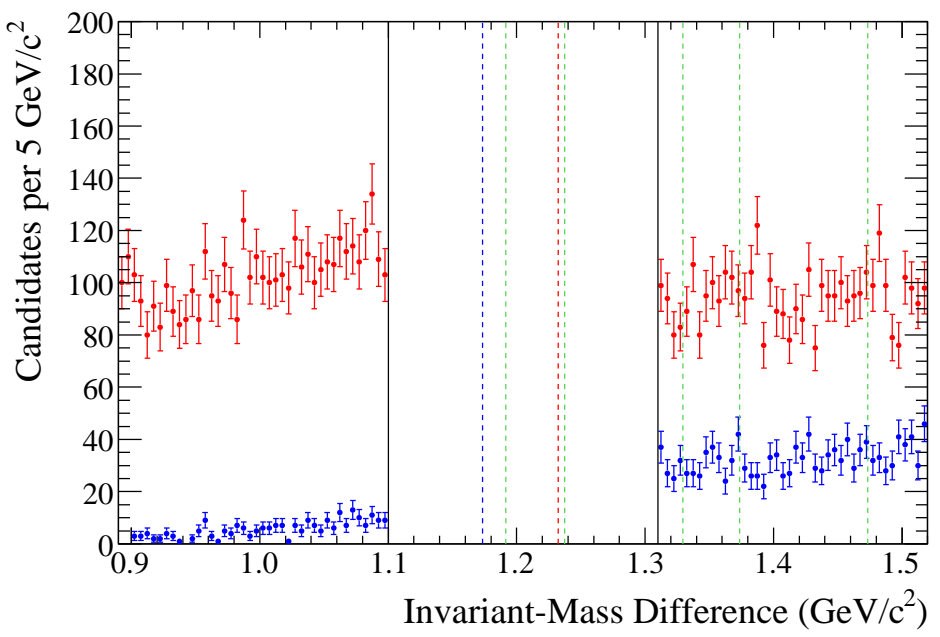

Figure 3.1: The $\Xi_{c c}^{+}$(red) and $\Xi_{c c}^{++}$(blue) mass-difference distributions $M\left(\left(p K^{-} \pi^{+}\right) K^{-} \pi^{+}\right)-$ $M\left(p K^{-} \pi^{+}\right)$and $M\left(\left(p K^{-} \pi^{+}\right) K^{-} \pi^{+}\right)-M\left(p K^{-} \pi^{+}\right)$, respectively. The blinded region is between the solid black vertical lines and the data are shown in side-band regions to the right and left. The $\Xi_{c c}^{+}$mass reported by SELEX is indicated by the dashed red vertical line. The $\Xi_{c c}^{++}$mass reported by SELEX is indicated by the dashed blue vertical line. The dashed green vertical lines, from left to right, are the theoretical masses from References [27], [29], [31], [28], and [30], respectively. These theoretical papers do not give any predictions for the mass splitting between the $\Xi_{c c}^{+}$and $\Xi_{c c}^{++}$ baryons.

signal.

I search for the $\Xi_{c c}^{+}$and $\Xi_{c c}^{++}$baryons using two different methods, both involving maximumlikelihood fits to the data. In one method, I search for $\Xi_{c c}^{+}$and $\Xi_{c c}^{++}$at their claimed masses of $(3.5187 \pm 0.0017) \mathrm{GeV} / c^{2}$ and $3.46 \mathrm{GeV} / c^{2}$, respectively. My second method is a search for $\Xi_{c c}^{+}$ and $\Xi_{c c}^{++}$throughout the $210 \mathrm{MeV} / c^{2}$-wide blinded mass-difference region. When searching at the claimed $\Xi_{c c}^{+}$and $\Xi_{c c}^{++}$masses, I allow a $10 \mathrm{MeV} / c^{2}$ range for the fitted signal mass, centered on the respective mass reported by the SELEX Collaboration, and I fit a $100 \mathrm{MeV} / c^{2}$ wide region centered at the same mass. When searching the entire $210 \mathrm{MeV} / c^{2}$ blinded region, I perform 21 separate fits where the fitted mass is allowed in sequential $10 \mathrm{MeV} / c^{2}$ ranges that, together, span the entire blinded region; these fits are also of $100 \mathrm{MeV} / c^{2}$ wide regions centered on each signal region. The fitted number of signal events $(N)$ is allowed to be either positive or negative. The fitted number of signal events and its error $\left(\sigma_{N}\right)$ are used to construct a significance measure, $N / \sigma_{N}$, for each maximum-likelihood fit to the data. When searching the entire blinded region, only the fit with the largest significance-measure is considered as a potential signal.

Searching a $210 \mathrm{MeV} / c^{2}$ wide region for a signal presents a higher probability of finding a background fluctuation that looks like a signal than searches of a $10 \mathrm{MeV} / c^{2}$ wide region. This is even true for searching a $10 \mathrm{MeV} / c^{2}$ wide region versus searching for a signal at one specific mass. The 
technique that I use to calculate the statistical significances of double-charm baryon signals accounts for this effect. To calculate the significance of any potential signal, toy Monte Carlo (TMC) studies are performed for each search using 1000 two-dimensional TMC samples. The TMC samples are generated according to the parameters extracted from the background-only fits to the data that will be described in Section 3.4. Searches for signals are performed on the TMC samples sets just as they are performed on data. The significance measures from each TMC study (1000 significance measures for each type of search) are used to create a discrete probability distribution function for each search. Each of these discrete functions can be integrated to yield the probability of finding, in a search applied to the data, any particular significance measure or less, under the hypothesis that there is no real signal in the data. These probabilities are the p-values (or true significances) for signals found in the data. The accuracy of these p-values is only limited by the number of TMC samples studied.

Efficiencies for reconstructing double-charm baryon candidates are calculated based on signalMC samples. Combining these efficiencies $(\epsilon)$ with signal yields $(N)$ and integrated luminosity (L) gives a product of the cross-section and branching fractions $(\mathcal{S})$ such as

$$
\mathcal{S}_{\Xi_{c c}^{+}}=\left(\frac{N}{\epsilon \mathrm{L}}\right)_{\Xi_{c c}^{+}}=\sigma\left(e^{+} e^{-} \rightarrow \Xi_{c c}^{+} X\right) \times \mathcal{B}\left(\Xi_{c c}^{+} \rightarrow \Lambda_{c}^{+} K^{-} \pi^{+}\right) \times \mathcal{B}\left(\Lambda_{c}^{+} \rightarrow p K^{-} \pi^{+}\right),
$$

where $X$ is the portion of each event that is not analyzed. Since the world average branching fraction $\mathcal{B}\left(\Lambda_{c}^{+} \rightarrow p K^{-} \pi^{+}\right)$has a $25 \%$ error [7], I also measure the $\Lambda_{c}^{+}$production cross-section times branching fraction,

$$
\mathcal{S}_{\Lambda_{c}}=\left(\frac{N}{\epsilon \mathrm{L}}\right)_{\Lambda_{c}}=\sigma\left(e^{+} e^{-} \rightarrow \Lambda_{c}^{+} X\right) \times \mathcal{B}\left(\Lambda_{c}^{+} \rightarrow p K^{-} \pi^{+}\right)
$$

This allows me to calculate a ratio such as

$$
\frac{\sigma\left(e^{+} e^{-} \rightarrow \Xi_{c c}^{+} X\right) \times \mathcal{B}\left(\Xi_{c c}^{+} \rightarrow \Lambda_{c}^{+} K^{-} \pi^{+}\right)}{\sigma\left(e^{+} e^{-} \rightarrow \Lambda_{c}^{+} X\right)}
$$

and eliminate the $25 \%$ uncertainty due to $\mathcal{B}\left(\Lambda_{c}^{+} \rightarrow p K^{-} \pi^{+}\right)$.

\subsection{Data and Monte-Carlo Samples}

For my double-charm baryon searches, I analyze a sample of $e^{+} e^{-}$events recorded with the BABAR Detector corresponding to an integrated luminosity of $232 \mathrm{fb}^{-1}$. I select a subsample of events that contain at least one $\Lambda_{c}^{+} \rightarrow p K^{-} \pi^{+}$candidate. This subsample has been used for several analyses besides my own. Any track considered to be from a proton, kaon, or pion must pass their respective VeryLoose likelihood criteria. The $\Lambda_{c}^{+}$candidates are formed by constraining each combination 


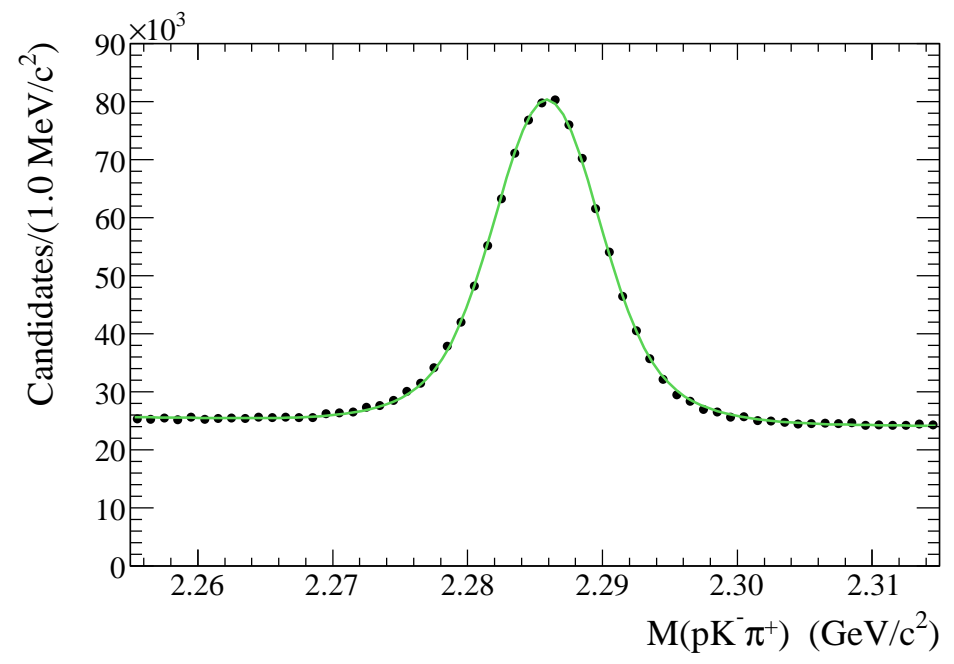

Figure 3.2: The $M\left(p K^{-} \pi^{+}\right)$invariant mass for $\Lambda_{c}^{+}$candidates in an integrated luminosity of $232 \mathrm{fb}^{-1}$. From the fit (green curve), there are approximately 1.5 million $\Lambda_{c}^{+}$baryons with a mass and width of about $2286 \mathrm{MeV} / c^{2}$ and $6 \mathrm{MeV}$, respectively.

of reconstructed tracks passing $p, K^{-}$, and $\pi^{+}$criteria to a common vertex. Given their measured trajectories and the uncertainties of the track parameters, a $\chi^{2}$ probability for the vertex-constrained fit is calculated. For an event to be included in the subsample, a fit of the $p K^{-} \pi^{+}$tracks must have a $\chi^{2}$ probability greater than 0.001 and an invariant mass between $2235 \mathrm{MeV} / c^{2}$ and $2335 \mathrm{MeV} / c^{2}$.

The $\Lambda_{c}^{+} \rightarrow p K^{-} \pi^{+}$subsample contains $17,718,614$ events $(0.57 \%$ of all the events corresponding to an integrated luminosity of $\left.232 \mathrm{fb}^{-1}\right)$. The $M\left(p K^{-} \pi^{+}\right)$invariant-mass distribution of $\Lambda_{c}^{+}$candidates, selected to meet criteria as described in Section 3.3, is shown in Figure 3.2. The distribution is fit with the sum of two Gaussian distribution and a linear background. From the fit, there are approximately 600 thousand $\Lambda_{c}^{+}$baryons with a mass and RMS width of about $2286 \mathrm{MeV} / \mathrm{c}^{2}$ and $6 \mathrm{MeV}$, respectively. The fitted mass and width here are not to be taken as final results; they are used solely as reference values for the remainder of the double-charm baryon analysis. The events studied in this analysis are a subsample of all events with $\Lambda_{c}^{+}$candidates. Additional $K^{-}$and $\pi^{+}$ candidates in each event (required to pass VeryLoose likelihood criteria) are combined with the $\Lambda_{c}^{+}$ candidates to form $\Xi_{c c}^{+} \rightarrow \Lambda_{c}^{+} K^{-} \pi^{+}$and $\Xi_{c c}^{++} \rightarrow \Lambda_{c}^{+} K^{-} \pi^{+} \pi^{+}$candidates. Each event is further studied only if there is at least one $\Xi_{c c}^{+}$or $\Xi_{c c}^{++}$candidate with invariant mass between $1.0 \mathrm{GeV} / c^{2}$ and $5.0 \mathrm{GeV} / c^{2}$.

Two samples of signal MC events are created, one based on a diquark model and one on a fragmentation model. Each of these models is meant to represent the true production of doublecharm baryons. About 114,000 fragmentation-model $\Xi_{c c}^{+}$events are simulated as well as 114,000 $\Xi_{c c}^{++}$events. About 10,000 diquark-model $\Xi_{c c}^{+}$events and 10,000 diquark-model $\Xi_{c c}^{++}$events are simulated. The fragmentation-model MC events are used to estimate signal event reconstruction 
efficiencies and invariant-mass resolutions, and to determine signal selection criteria. The diquarkmodel MC samples are created to assess qualitative differences between the two models and are also used to determine signal selection criteria. $\Xi_{c c}^{+}$and $\Xi_{c c}^{++}$baryons are generated with masses of $3519 \mathrm{MeV} / \mathrm{c}^{2}$ and $3460 \mathrm{MeV} / \mathrm{c}^{2}$, respectively, and with zero natural width. Monte Carlo events are reconstructed based on the output of the GEANT4 BABAR Detector simulation. I process the signal-MC samples using the same reconstruction software, and track and candidate requirements as the data. The reconstructed signal MC events can be compared to the simulated event that is the input to the GEANT4 detector simulation. When a reconstructed track or candidate corresponds with a real simulated track or candidate, it is said to be "truth matched".

A charm diquark is a theoretical construct where two charm quarks or two anti-charm quarks are loosely bond to one another through the QCD force. This simplifies the modeling of two charm quarks to the modeling just one diquark object. It also simplifies the modeling of the three quarks in a baryon to the modeling just two objects, analogous to the two quarks in a meson. In the diquark model, a pair of charm diquarks $(c c$ and $\bar{c} \bar{c})$ is generated from an $e^{+} e^{-}$annihilation. The $c c$ and $\bar{c} \bar{c}$ diquarks are produced in the simulation with the angular distribution predicted by Braguta and Chalov [25],

$$
P_{c c}(\theta)=1-0.35 \cos ^{2}(\theta)
$$

where $\theta$ is the polar angle with respect to the beam axis. These diquarks are then passed through the Jetset7 .4 fragmentation simulation. Here the diquarks can form double-charm baryons or other charmed hadrons. I keep only those events that contain at least one double-charm baryon.

In the fragmentation model, the nominal $e^{+} e^{-} \rightarrow \gamma \rightarrow c \bar{c}$ simulation is used, but the Jetset7.4 fragmentation process is modified. The probability for a sea diquark from the fragmentation process to contain a charm quark is increased from $0 \%$ to $50 \%$. A diquark that contains a charm quark can then hadronize with a primary charm quark (directly from the $e^{+} e^{-}$annihilation), forming a double-charm baryon. I keep only those event that contain at least one double-charm baryon.

The two major differences between the diquark and fragmentation models are the generated polar angle $\left(\theta_{c m}\right)$ and the momentum $\left(p^{*}\right)$ distributions of the double-charm baryons measured in the $e^{+} e^{-}$center-of-mass frame. The differences in the $\theta_{c m}$ distributions are illustrated in Figures 3.3 and 3.4, and the differences in the $p^{*}$ distributions are illustrated in Figures 3.5 and 3.6. The diquark model produces $c c$ diquarks with an angular distribution that peaks at $90^{\circ}$ to the beam line in the $e^{+} e^{-}$center-of-mass frame. In contrast, the fragmentation model produces primary $c \bar{c}$ quarks with a distribution that peaks along the beam line. The diquark model also produces double-charm baryons with a higher $p^{*}$ distribution than the fragmentation model. 


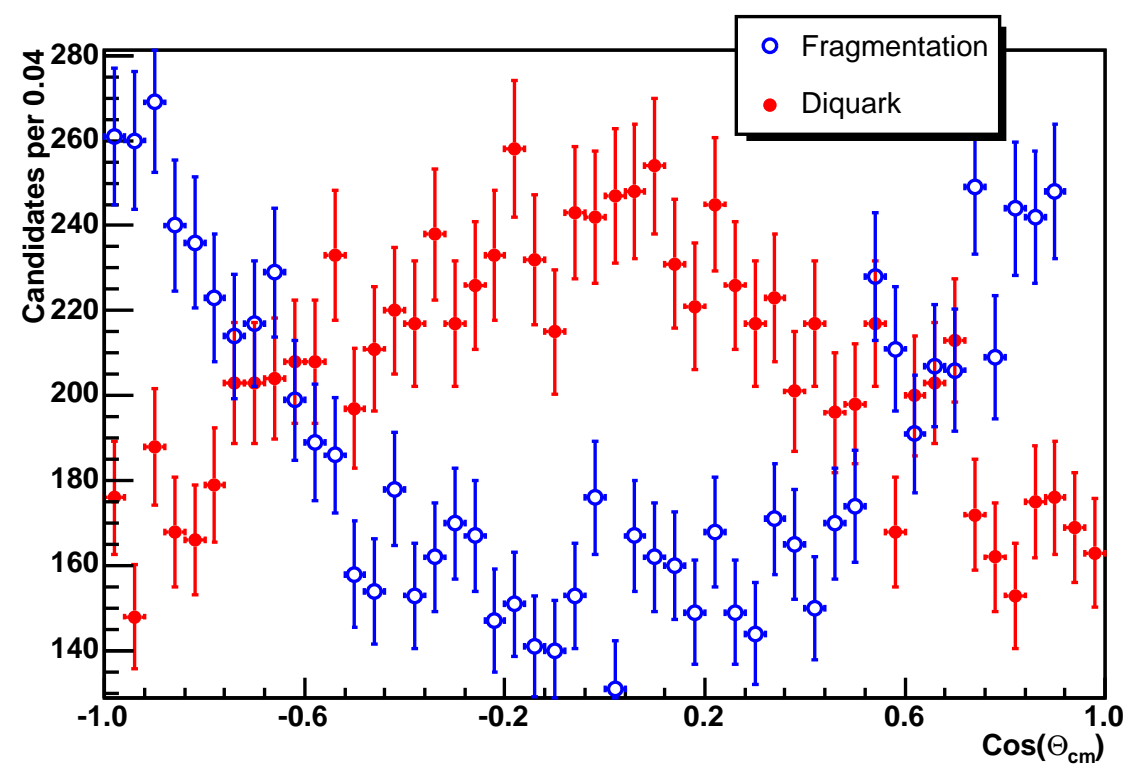

Figure 3.3: Simulated center-of-mass polar angle distributions of $\Xi_{c c}^{+}$. The distribution from the diquark-model peaks at $90^{\circ}$ to the beam line while that for the fragmentation-model peaks along to the beam line.

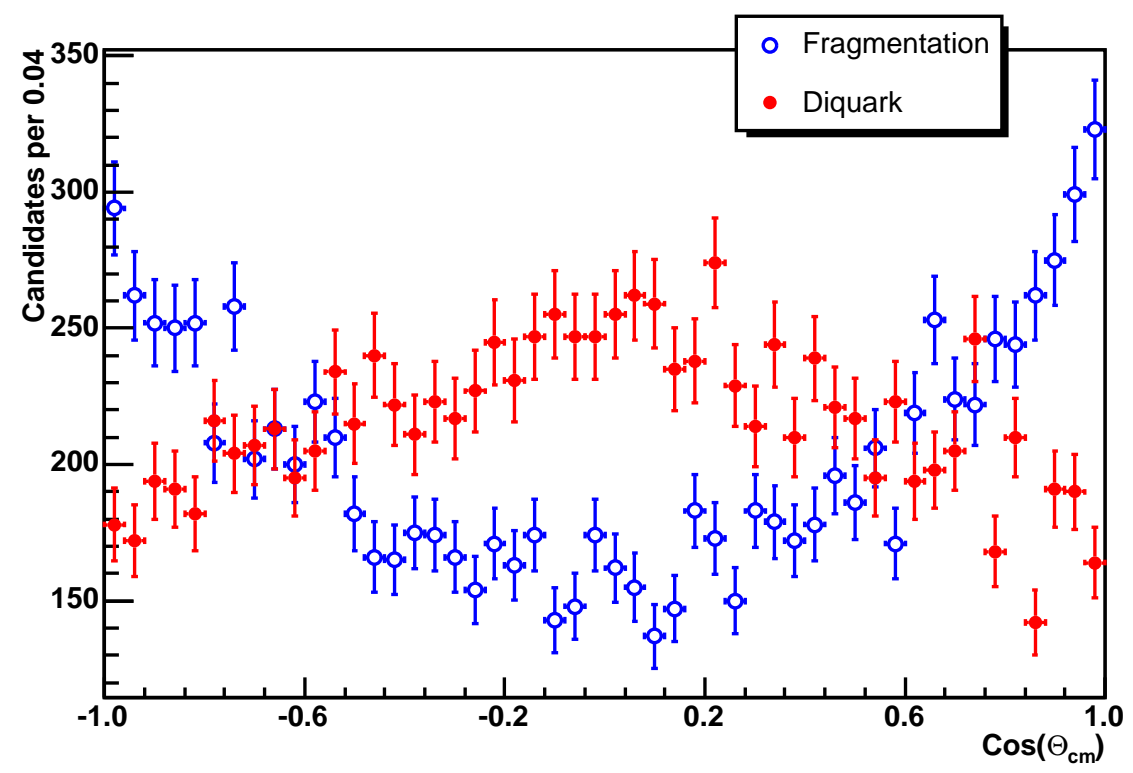

Figure 3.4: Simulated center-of-mass polar angle distributions of $\Xi_{c c}^{++}$. The distribution from the diquark-model peaks at $90^{\circ}$ to the beam line while that for the fragmentation-model peaks along to the beam line. 


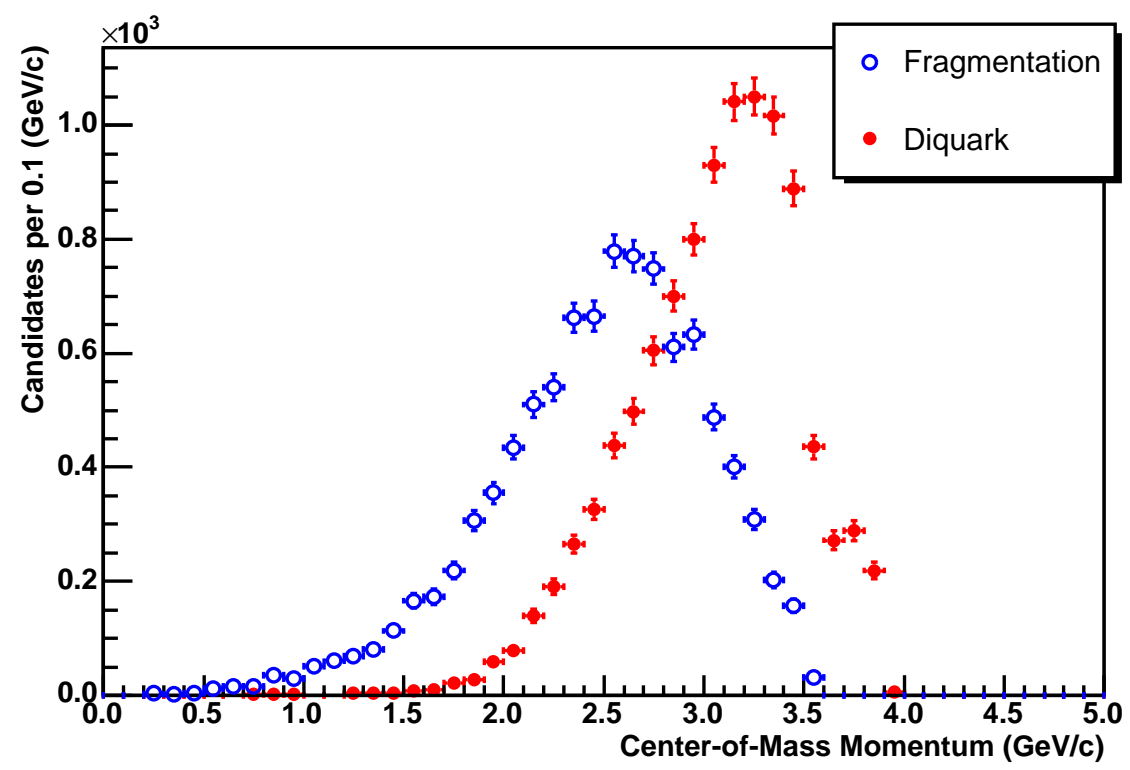

Figure 3.5: Simulated $p^{*}$ distributions for $\Xi_{c c}^{+}$. The distribution for the diquark-model has a higher average momentum than the fragmentation-model.

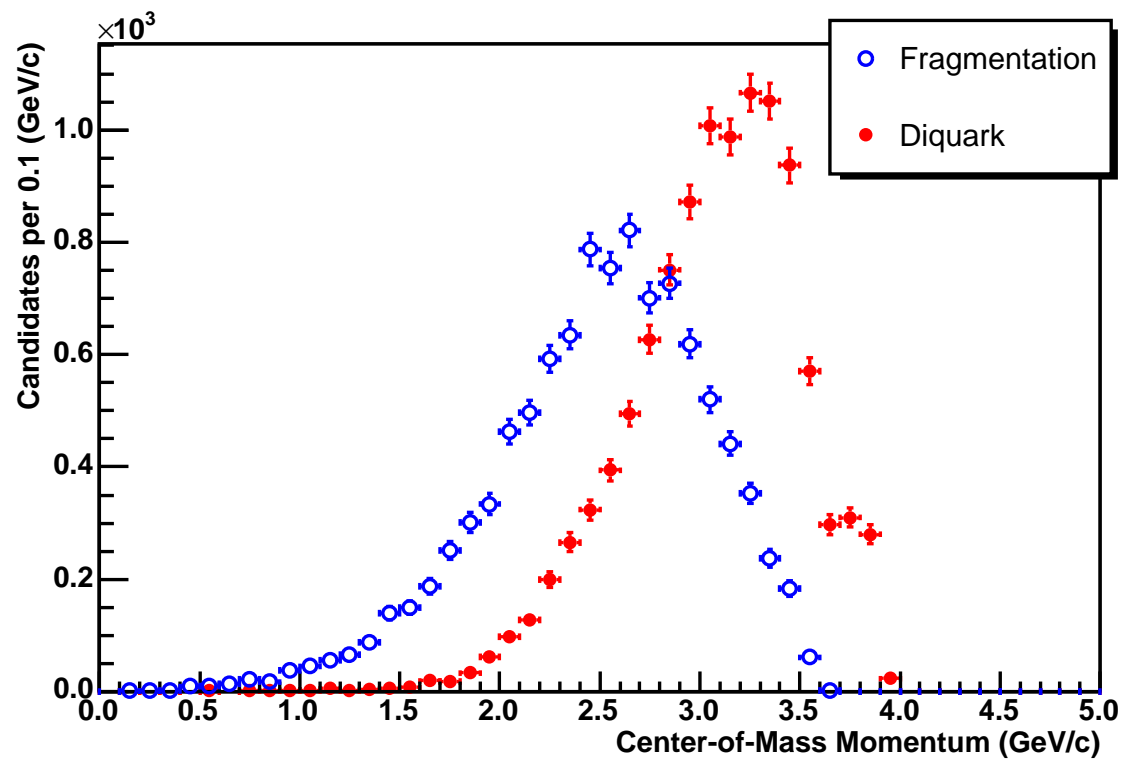

Figure 3.6: Simulated $p^{*}$ distributions for $\Xi_{c c}^{++}$. The distribution for the diquark-model has a higher average momentum than the fragmentation-model. 
Table 3.2: Variables used in the optimization of selection criteria. Each variable has different levels of discrimination, which are listed along with the number of levels (multiplicity). The total number of different sets of selection criteria is tabulated at the bottom.

\begin{tabular}{|c|c|c|}
\hline Variable & Criteria Levels & Multiplicity \\
\hline Track Quality & ChargedTracks | VeryLoose | Loose | Tight & $\times 4$ \\
\hline Acceptance Angle & No Requirement $\mid-0.825<\cos \theta<0.917$ & $\times 2$ \\
\hline$\Xi_{c c}$ Vertex Prob. & $>0.1 \%|>0.5 \%|>1.0 \% \mid>1.5 \%$ & $\times 4$ \\
\hline$\Lambda_{c}$ Vertex Prob. & $>0.1 \%|>0.5 \%|>1.0 \% \mid>1.5 \%$ & $\times 4$ \\
\hline$p_{\Lambda_{c}} \mathrm{PID}$ & VeryLoose | Loose | Tight | VeryTight & $\times 4$ \\
\hline$K_{\Lambda_{c}} \mathrm{PID}$ & Loose | Tight | VeryTight & $\times 4$ \\
\hline$\pi_{\Lambda_{c}} \mathrm{PID}$ & Loose | Tight & $\times 4$ \\
\hline$K_{\Xi_{c c}} \mathrm{PID}$ & Loose | Tight & $\times 4$ \\
\hline$\pi \Xi_{c c} \mathrm{PID}$ & Loose | Tight & $\times 4$ \\
\hline$\pi_{\Xi_{c c}^{++}} \mathrm{PID}$ & VeryLoose | Loose | Tight | VeryTight & $\times 4$ \\
\hline \multirow{2}{*}{\multicolumn{2}{|c|}{$\begin{array}{l}\text { Total Number of Cut Combinations for } 5 \text { Daughter Tracks } \\
\text { Total Number of Cut Combinations for } 6 \text { Daughter Tracks }\end{array}$}} & 131,072 \\
\hline & & 524,228 \\
\hline
\end{tabular}

\subsection{Candidate Selection}

During the event reconstruction process, tracks and candidates are required to pass some basic criteria. These reconstruction criteria reduce the number of track combinations considered to a manageable level without being so stringent as to significantly reduce signal reconstruction efficiencies. Millions of $\Xi_{c c}^{+}$and $\Xi_{c c}^{++}$candidates pass the event reconstruction criteria. This is due to the combinatorics of joining five or six loosely identified tracks when there can be many more than six tracks in an event. In order to reduce this very large combinatoric background and increase sensitivity to signal, more stringent criteria are required of each candidate. These new criteria are chosen to maximize the expected sensitivity of the search under the hypothesis that there is no signal in the data.

\subsubsection{Maximizing Significance}

Particle identification, track quality, vertex $\chi^{2}$ probability, and kinematic quantities are used to distinguish background candidates from signal candidates. These variables and their levels of discrimination are listed in Table 3.2. The acceptance-angle is the range of polar angle $\theta$ with respect to the $z$-axis for which tracks are allowed. The significance of a signal is estimated for every possible combination of discrimination levels outlined in Table 3.2. The sensitivities for these 131,057 (524,228 for six daughter tracks) different combinations are estimated by $S / \sqrt{B}$, where $S$ is the estimated amount of signal and $B$ is the estimated amount of background. Each amount of signal, $S$, is estimated using the diquark-model and fragmentation-model MC samples. MC signal candidates 


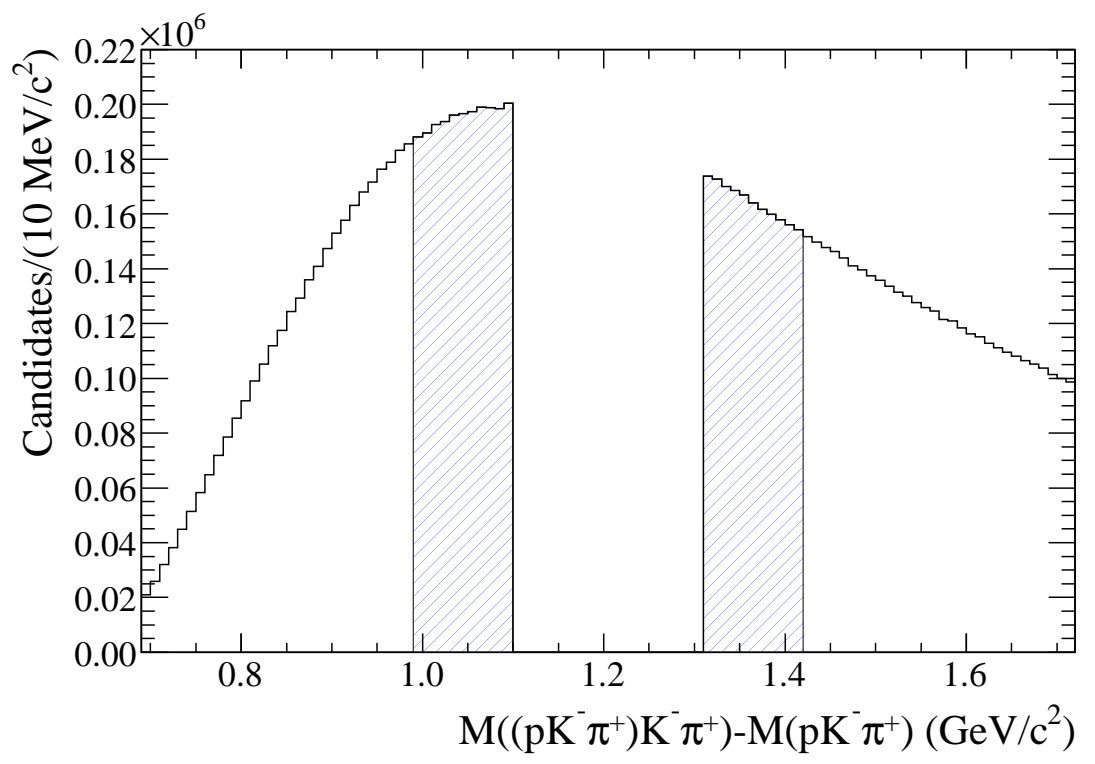

Figure 3.7: Distribution of $M\left(\left(p K^{-} \pi^{+}\right) K^{-} \pi^{+}\right)-M\left(p K^{-} \pi^{+}\right)$for all $\Xi_{c c}^{+}$candidates. The hatched regions correspond to candidates used for the background estimate $B$. The $\Xi_{c c}^{+}$signal region is blinded.

that pass a set of discrimination levels, fall within a blinded invariant-mass region, and correspond to actual simulated $\Xi_{c c}^{+}$and $\Xi_{c c}^{++}$baryons, are counted as signal. To compensate for differences between MC efficiencies and data efficiencies for PID and track quality criteria, weighting tables are used to weight each passed candidate. As an estimate of the amount of background, $B$, data side bands in invariant-mass difference are used. These mass-difference sidebands are $210 \mathrm{MeV} / \mathrm{c}^{2}$ wide and on both sides of the blinded search region. Figure 3.7 and Figure 3.8 depict the side-band regions and the blinded regions used for $\Xi_{c c}^{+}$and $\Xi_{c c}^{++}$, respectively. Candidates that pass a set of selection criteria and are in these side-band regions are counted in the background estimate.

\subsubsection{Optimization of Selection Criteria}

The set of criteria for the variables listed in Table 3.2 that maximizes the estimated significance is determined. This maximization is done separately for six different ranges of $p^{*}(0.5 \mathrm{GeV} / c$ wide ranges from $1.0 \mathrm{GeV} / c$ to $4.0 \mathrm{GeV} / c$ ) in addition to being done separately for each type of double-charm baryon and each simulation model. The optimization is done for ranges of $p^{*}$ because the signal MC and data side-bands have significantly different in $p^{*}$ distributions, and the true $p^{*}$ distribution for double-charm baryons is unknown. Reconstructed $p^{*}$ distributions for signal MC candidates and for data candidates in the mass-difference side bands are shown in Figure 3.9 and Figure 3.10.

The optimal criteria are determined using two-dimensional density plots where the estimated 


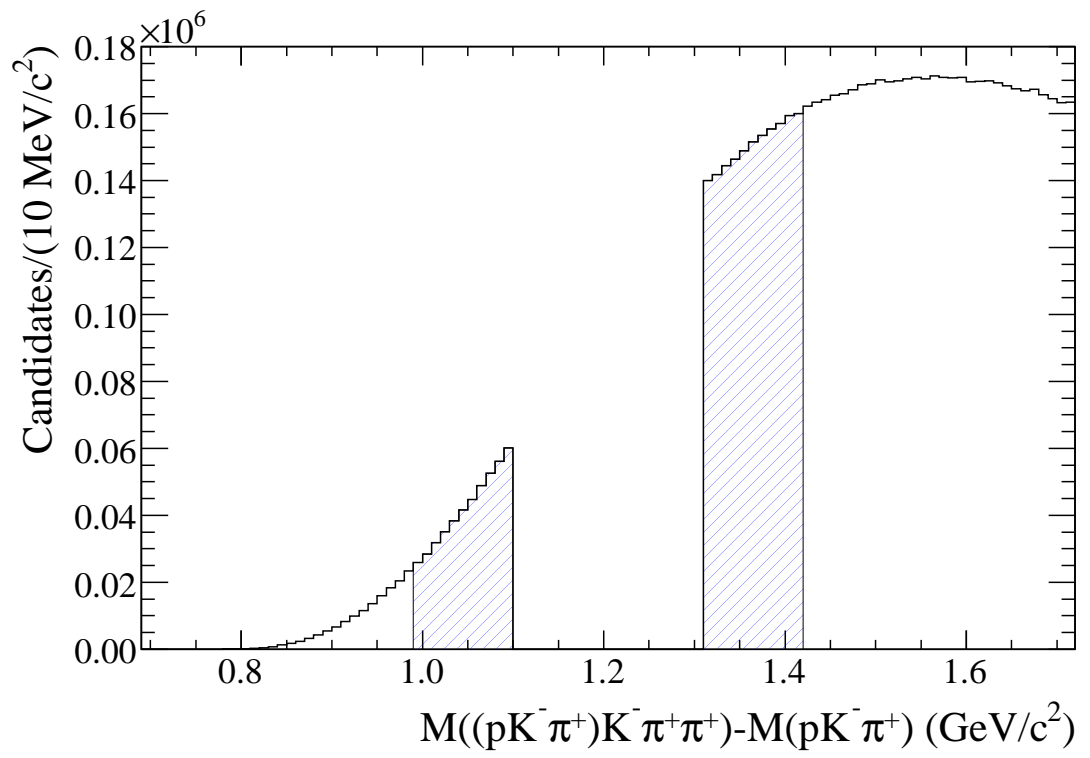

Figure 3.8: Distribution of $M\left(\left(p K^{-} \pi^{+}\right) K^{-} \pi^{+} \pi^{+}\right)-M\left(p K^{-} \pi^{+}\right)$for all $\Xi_{c c}^{++}$candidates. The hatched regions correspond to candidates used for the background estimate $B$. The $\Xi_{c c}^{++}$signal region is blinded.

significances, $S / \sqrt{B}$, for every set of criteria are plotted versus one variable at a time. For example, significance versus proton PID level is shown in Figure 3.11 for $\Xi_{c c}^{+}$candidates generated via the diquark method, with $3.0 \mathrm{GeV} / c<p^{*}<3.5 \mathrm{GeV} / c$. The colors represent the density of the 131,057 sets of selection criteria described in Table 3.2. There is much that can be seen in the structure of such density plots. Sets of criteria that provide similar significance create high density regions. When the significances of the criteria sets are plotted versus a highly discriminating variable, there are visible differences in the maximum significance for each criteria level of that variable. Other variables that are highly discriminating but are not explicitly plotted, create regions of high density that are widely spaced in significance. From the various density bands created by the thousands of sets of criteria, one can see which discrimination variables have a substantial effect on the significance. In Figure 3.11, a line is drawn through the VeryTight bin because it has been determined to have the optimal significance. The optimal criteria is defined to be that which results in the largest $\mathcal{F}_{i}=M_{i}+A_{i}$, where $M_{i}$ is the maximum significance in bin $i$ and $A_{i}$ is the average significance for all the sets of selection criteria in bin $i$. The values of $\mathcal{F}_{i}$ are used to find the optimal criteria because the $M_{i}$ are often too similar to reliably differentiate between selection criteria.

Tables 3.3 and 3.4 list the criteria that give the largest $\mathcal{F}$ (the optimal significance) for $\Xi_{c c}^{+}$and $\Xi_{c c}^{++}$, respectively. As can be seen from these tables, most variables are not consistent and their optimal levels fluctuate randomly from one $p^{*}$ bin to the next. This is because there are small differences in significance between the criteria levels in these variables and statistical fluctuations 


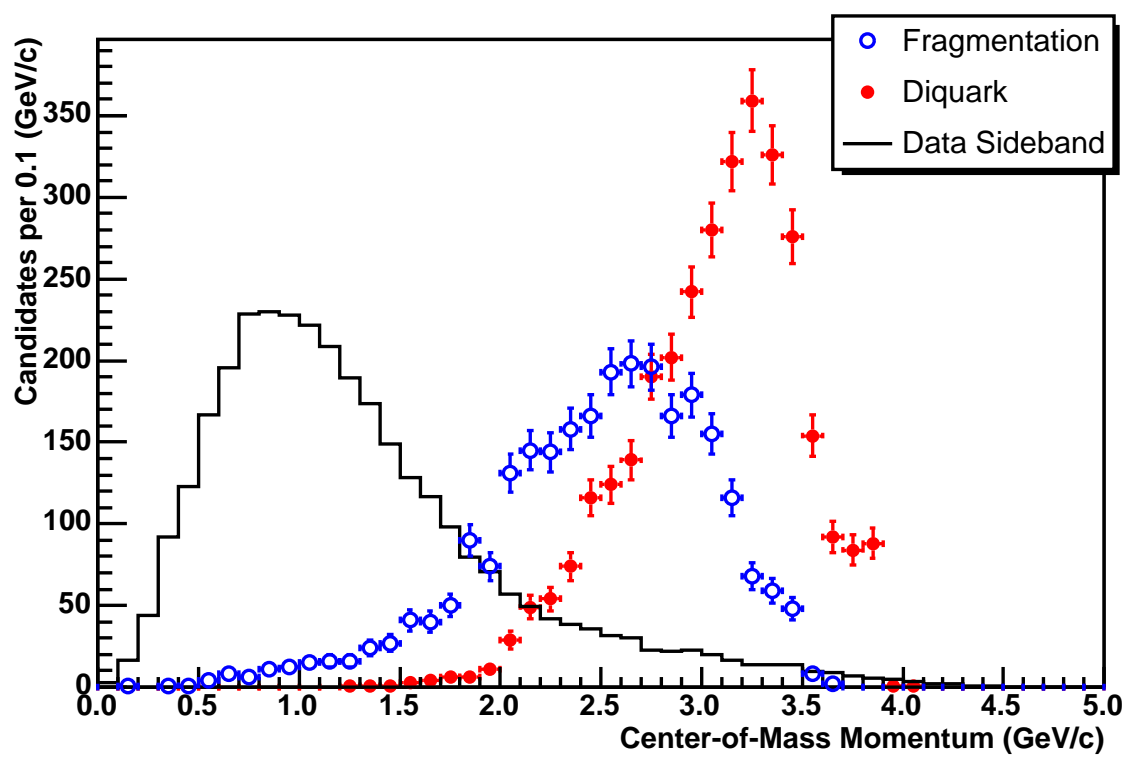

Figure 3.9: Distributions of $p^{*}$ from mass-difference side-band data of $\Xi_{c c}^{+}$candidates and truthmatched $\Xi_{c c}^{+} \mathrm{MC}$ candidates for both the diquark-model and the fragmentation-model simulations. The side-band data are scaled arbitrarily.

from the finite data and MC samples. Track acceptance angle and proton PID variables provide the most robust discrimination. Based on the two MC models, the optimal levels of discrimination have only a slight dependences on the $p^{*}$ range. For this reason, selection criteria are fixed to be the same for the entire range of $p^{*}$. Wherever there is an observed $p^{*}$ dependence, I use the selection criteria that are optimal for higher $p^{*}$ candidates. I also base the final selection criteria on the average between the diquark-model and fragmentation-model criteria listed in Tables 3.3 and 3.4. The final selection criteria are listed Table 3.5. Overall, the final PID discrimination levels are more stringent for the $\Xi_{c c}^{++}$candidates than for the $\Xi_{c c}^{+}$candidates. This is most likely because the additional $\pi^{+}$ in the $\Xi_{c c}^{++}$search contributes additional combinatorial background, which can be suppressed with more stringent PID requirements. All track-quality requirements are Loose because it is the only track-quality criteria for which MC-data efficiency correction tables are available, and because the track quality levels showed little difference in significance.

\subsubsection{Selection of $p^{*}$ Requirement}

A $p^{*}$ requirement is motivated by the knowledge that other charm baryons, such as the $\Lambda_{c}^{+}$, have $p^{*}$ distributions that peak at higher $p^{*}$ than combinatoric background. To improve the sensitivity of the search for $\Xi_{c c}^{+}$and $\Xi_{c c}^{++}$baryons, a requirement for the minimum allowed $p^{*}$ of signal candidates is chosen. All searches are also performed without using a $p^{*}$ requirement, because such results are more 


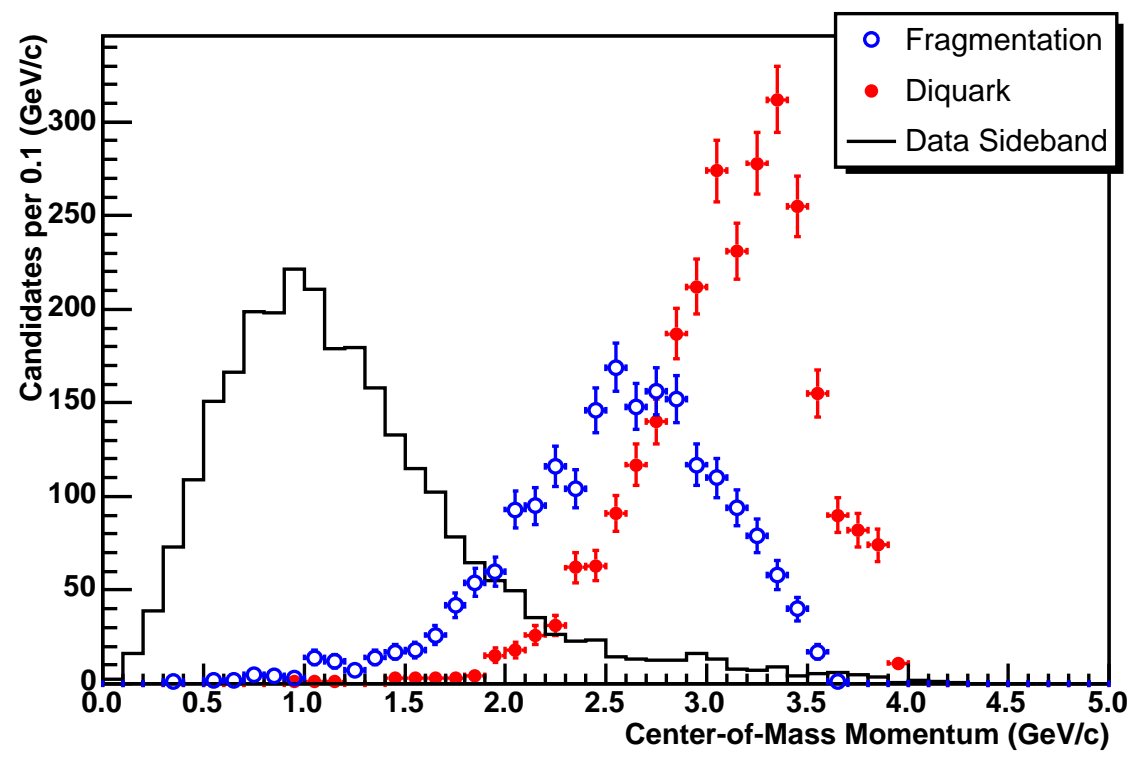

Figure 3.10: Distributions of $p^{*}$ from mass-difference side-band data of $\Xi_{c c}^{++}$candidates and truthmatched $\Xi_{c c}^{++} \mathrm{MC}$ candidates for both the diquark-model and the fragmentation-model simulations. The side-band data are scaled arbitrarily.

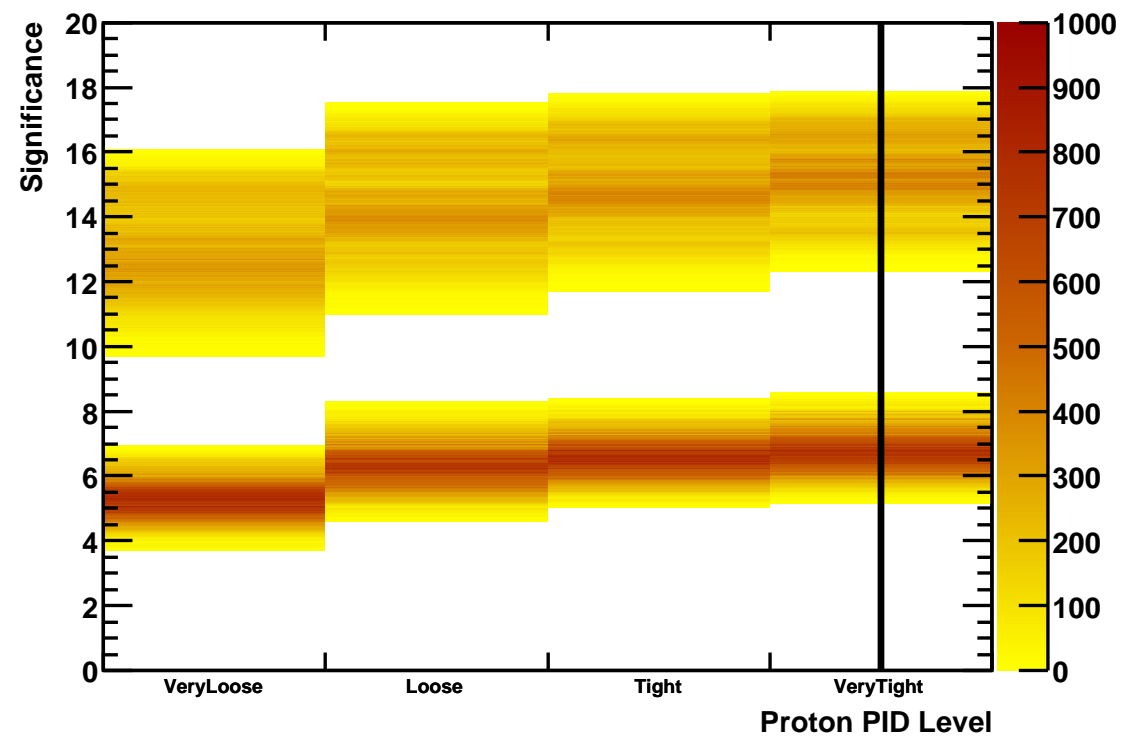

Figure 3.11: Sets of selection criteria from Table 3.2 shown in a two-dimensional density plot of significance versus proton PID level for $\Xi_{c c}^{+}$candidates with $3.0 \mathrm{GeV} / c<p^{*}<3.5 \mathrm{GeV} / c$ generated via the diquark method. The colors represent the density of the 131,057 sets of selection criteria described in Table 3.2. The vertical line in the VeryTight bin indicates that this bin has been found to have the largest maximum plus average significance $(\mathcal{F})$ of all the proton PID levels. The bin with the largest $\mathcal{F}$ is chosen as the optimal level of discrimination for that variable. 
Table 3.3: Levels of discrimination for optimal estimated significance of $\Xi_{c c}^{+}$in ranges of $p^{*}$. In each pair of discrimination levels separated by a vertical line, the first corresponds to the diquark-model simulation and the second corresponds to the fragmentation-model simulation. Vertex probabilities are $>X \%$. VT, T, L, VL, and CT stand for VeryTight, Tight, Loose, VeryLoose, and ChargedTracks, respectively.

\begin{tabular}{lcccccc}
\hline$p^{*}(\mathrm{GeV} / c)$ & $1.0-1.5$ & $1.5-2.0$ & $2.0-2.5$ & $2.5-3.0$ & $3.0-3.5$ & $3.5-4.0$ \\
\hline \hline Track Quality & $\mathrm{T} \mid \mathrm{CT}$ & $\mathrm{L} \mid \mathrm{T}$ & $\mathrm{T} \mid \mathrm{T}$ & $\mathrm{T} \mid \mathrm{T}$ & $\mathrm{T} \mid \mathrm{T}$ & $\mathrm{T} \mid \mathrm{T}$ \\
\hline Acceptance. $\theta$ & Yes $\mid$ Yes & Yes $\mid$ Yes & Yes $\mid$ Yes & Yes $\mid$ Yes & Yes $\mid$ Yes & Yes $\mid$ No \\
\hline$\Xi_{c c}^{+}$Vertex Prob. & $1.5 \mid 1.0$ & $1.0 \mid 1.5$ & $0.5 \mid 1.5$ & $0.5 \mid 1.5$ & $1.5 \mid 0.1$ & $1.5 \mid 1.5$ \\
\hline$\Lambda_{c}$ Vertex Prob. & $1.5 \mid 1.5$ & $1.5 \mid 1.0$ & $1.0 \mid 0.5$ & $1.5 \mid 1.5$ & $0.5 \mid 1.0$ & $0.1 \mid 1.5$ \\
\hline$p_{\Lambda_{c}}$ PID & $\mathrm{L} \mid \mathrm{VT}$ & $\mathrm{VT} \mid \mathrm{VT}$ & $\mathrm{VT} \mid \mathrm{VT}$ & $\mathrm{VT} \mid \mathrm{VT}$ & $\mathrm{VT} \mid \mathrm{VT}$ & $\mathrm{VT} \mid \mathrm{VT}$ \\
\hline$K_{\Lambda_{c}}$ PID & $\mathrm{VT} \mid \mathrm{VT}$ & $\mathrm{L} \mid \mathrm{L}$ & $\mathrm{L} \mid \mathrm{VT}$ & $\mathrm{L} \mid \mathrm{VT}$ & $\mathrm{T} \mid \mathrm{L}$ & $\mathrm{VT} \mid \mathrm{VT}$ \\
\hline$\pi_{\Lambda_{c}}$ PID & $\mathrm{VT} \mid \mathrm{VL}$ & $\mathrm{VT} \mid \mathrm{VT}$ & $\mathrm{L} \mid \mathrm{VT}$ & $\mathrm{L} \mid \mathrm{L}$ & $\mathrm{L} \mid \mathrm{T}$ & $\mathrm{T} \mid \mathrm{VT}$ \\
\hline$K_{\Xi_{c c}}$ PID & $\mathrm{VT} \mid \mathrm{L}$ & $\mathrm{VT} \mid \mathrm{VT}$ & $\mathrm{VT} \mid \mathrm{VT}$ & $\mathrm{VT} \mid \mathrm{T}$ & $\mathrm{T} \mid \mathrm{T}$ & $\mathrm{VT} \mid \mathrm{VT}$ \\
\hline$\pi_{\Xi_{c c}} \mathrm{PID}$ & $\mathrm{VT} \mid \mathrm{T}$ & $\mathrm{T} \mid \mathrm{VT}$ & $\mathrm{T} \mid \mathrm{L}$ & $\mathrm{L} \mid \mathrm{T}$ & $\mathrm{L} \mid \mathrm{T}$ & $\mathrm{L} \mid \mathrm{T}$ \\
\hline
\end{tabular}

Table 3.4: Levels of discrimination for optimal estimated significance of $\Xi_{c c}^{++}$in ranges of $p^{*}$. In each pair of discrimination levels separated by a vertical line, the first corresponds to the diquark-model simulation and the second corresponds to the fragmentation-model simulation. Vertex probabilities are $>X \%$. VT, T, L, VL, and CT stand for VeryTight, Tight, Loose, VeryLoose, and ChargedTracks, respectively.

\begin{tabular}{lcccccc}
\hline$p^{*}(\mathrm{GeV} / c)$ & $1.0-1.5$ & $1.5-2.0$ & $2.0-2.5$ & $2.5-3.0$ & $3.0-3.5$ & $3.5-4.0$ \\
\hline \hline Track Quality & $\mathrm{T} \mid \mathrm{T}$ & $\mathrm{VL} \mid \mathrm{L}$ & $\mathrm{L} \mid \mathrm{VL}$ & $\mathrm{T} \mid \mathrm{VL}$ & $\mathrm{T} \mid \mathrm{VL}$ & $\mathrm{T} \mid \mathrm{VL}$ \\
\hline Acceptance. $\theta$ & $\mathrm{Yes} \mid \mathrm{Yes}$ & Yes $\mid$ Yes & Yes $\mid$ Yes & Yes $\mid$ Yes & Yes $\mid$ Yes & Yes $\mid$ Yes \\
\hline$\Xi_{c c}^{++}$Vertex Prob. & $1.0 \mid 1.5$ & $1.5 \mid 1.5$ & $1.0 \mid 1.5$ & $0.5 \mid 1.5$ & $0.5 \mid 1.0$ & $1.0 \mid 1.0$ \\
\hline$\Lambda_{c}$ Vertex Prob. & $0.1 \mid 1.5$ & $1.0 \mid 0.1$ & $0.5 \mid 1.0$ & $1.0 \mid 1.0$ & $0.1 \mid 1.0$ & $0.1 \mid 1.5$ \\
\hline$p_{\Lambda_{c}}$ PID & $\mathrm{T} \mid \mathrm{T}$ & $\mathrm{VT} \mid \mathrm{VT}$ & $\mathrm{VT} \mid \mathrm{VT}$ & $\mathrm{VT} \mid \mathrm{VT}$ & $\mathrm{VT} \mid \mathrm{VT}$ & $\mathrm{T} \mid \mathrm{T}$ \\
\hline$K_{\Lambda_{c}} \mathrm{PID}$ & $\mathrm{VT} \mid \mathrm{VL}$ & $\mathrm{VT} \mid \mathrm{VT}$ & $\mathrm{VT} \mid \mathrm{VT}$ & $\mathrm{VT} \mid \mathrm{VT}$ & $\mathrm{T} \mid \mathrm{L}$ & $\mathrm{VT} \mid \mathrm{VT}$ \\
\hline$\pi_{\Lambda_{c}} \mathrm{PID}$ & $\mathrm{VT} \mid \mathrm{VT}$ & $\mathrm{VT} \mid \mathrm{VT}$ & $\mathrm{T} \mid \mathrm{L}$ & $\mathrm{T} \mid \mathrm{T}$ & $\mathrm{T} \mid \mathrm{T}$ & $\mathrm{T} \mid \mathrm{T}$ \\
\hline$K_{\Xi_{c c}} \mathrm{PID}$ & $\mathrm{VT} \mid \mathrm{VT}$ & $\mathrm{VT} \mid \mathrm{VT}$ & $\mathrm{VT} \mid \mathrm{VT}$ & $\mathrm{VT} \mid \mathrm{VT}$ & $\mathrm{VT} \mid \mathrm{VT}$ & $\mathrm{VT} \mid \mathrm{VT}$ \\
\hline$\pi_{\Xi_{c c}}^{1} \mathrm{PID}$ & $\mathrm{VT} \mid \mathrm{VT}$ & $\mathrm{VT} \mid \mathrm{VT}$ & $\mathrm{VT} \mid \mathrm{VT}$ & $\mathrm{VT} \mid \mathrm{VT}$ & $\mathrm{VT} \mid \mathrm{VT}$ & $\mathrm{VT} \mid \mathrm{VT}$ \\
\hline$\pi_{\Xi_{c c}}^{2} \mathrm{PID}$ & $\mathrm{VT} \mid \mathrm{VT}$ & $\mathrm{VT} \mid \mathrm{VT}$ & $\mathrm{VT} \mid \mathrm{VT}$ & $\mathrm{T} \mid \mathrm{VT}$ & $\mathrm{VT} \mid \mathrm{VT}$ & $\mathrm{T} \mid \mathrm{VT}$ \\
\hline
\end{tabular}


Table 3.5: Final selection criteria for double-charm baryon searches.

\begin{tabular}{lcc}
\hline & $\Xi_{c c}^{+}$ & $\Xi_{c c}^{++}$ \\
\hline \hline Track Quality & Loose & Loose \\
Acceptance Angle & Yes & Yes \\
$\Xi_{c c}$ Vertex Prob. & $>1.0 \%$ & $>1.0 \%$ \\
$\Lambda_{c}$ Vertex Prob. & $>1.0 \%$ & $>1.0 \%$ \\
$p_{\Lambda_{c}}$ PID & VeryTight & VeryTight \\
$K_{\Lambda_{c}}$ PID & Tight & VeryTight \\
$\pi_{\Lambda_{c}}$ PID & Loose & Tight \\
$K_{\Xi_{c c}}$ PID & Tight & VeryTight \\
$\pi_{\Xi_{c c}}^{1}$ PID & Loose & VeryTight \\
$\pi_{\Xi_{c c}}^{2}$ PID & - & VeryTight \\
\hline \hline
\end{tabular}

readily comparable to theoretical predictions. There are no theoretically predicted $p^{*}$ distributions for $\Xi_{c c}^{+}$or $\Xi_{c c}^{++}$, so the $p^{*}$ requirement is chosen based on $p^{*}$ distributions in the signal-MC samples. The fragmentation-model and diquark-model $p^{*}$ distributions in Figures 3.9 and 3.10 show that the MC simulations reasonably model higher $p^{*}$ distributions for signal candidates than there is from side-band data. The best $p^{*}$ requirement is determined based on estimated significance, $S / \sqrt{B}$, as described in Section 3.3.1. $S$ and $B$, however, are determined after all other selection criteria have been applied. The minimum allowed $p^{*}$ is varied in $0.1 \mathrm{GeV} / c^{2}$ steps to find the maximum estimated significance. The significances are plotted in Figures 3.12 through 3.15 as functions of minimum $p^{*}$. The optimal minimum $p^{*}$ for the fragmentation-model is lower than that for the diquark-model. As seen in Figures 3.12 through 3.15, there is a smaller reduction in estimated significance for the diquark-model if the optimal fragmentation-model $p^{*}$ requirement is used, compared to the converse. For this reason the fragmentation-model signal-MC sample is used in determining the minimum allowed $p^{*}$. As a $p^{*}$ requirement, I select candidates with $p^{*}$ greater than $2.3 \mathrm{GeV} / c$.

\subsubsection{Multiple Candidates per Event}

For many events, in both the data and signal-MC samples, there is more than one candidate that passes the selection criteria. Table 3.6 lists the fraction of signal-MC events with multiple candidates in the mass-difference signal region. The average number of candidates per event passing the selection criteria is also listed in Table 3.6. The values in Table 3.6 are calculated without any $p^{*}$ requirement.

Choosing one candidate per event only increases the sensitivity of a search if most of the time the correct candidate can be chosen. No selection criterion, besides $p^{*}$, provides substantial discrimination between the correct and incorrect candidates in the signal-MC samples. Since the $p^{*}$ discrimination power is dependent on how the signal-MC samples are generated, the value of $p^{*}$ is 


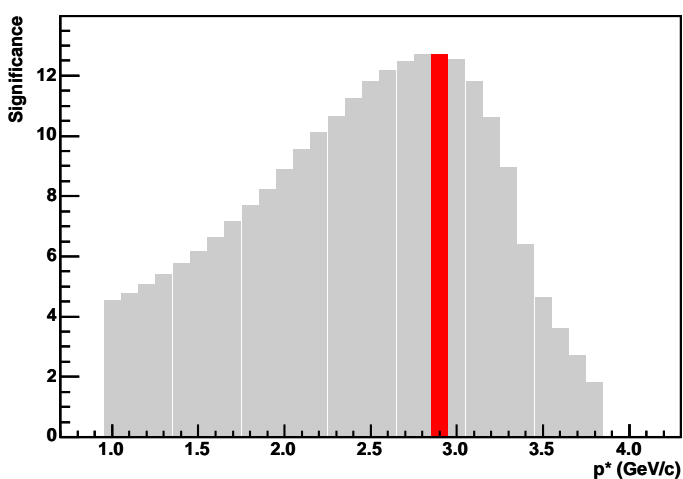

Figure 3.12: The estimated significance versus minimum allowed $p^{*}$ for $\Xi_{c c}^{+}$using the diquarkmodel and side-band data. The red bar indicates the minimum allowed $p^{*}$ resulting in the highest estimated significance.

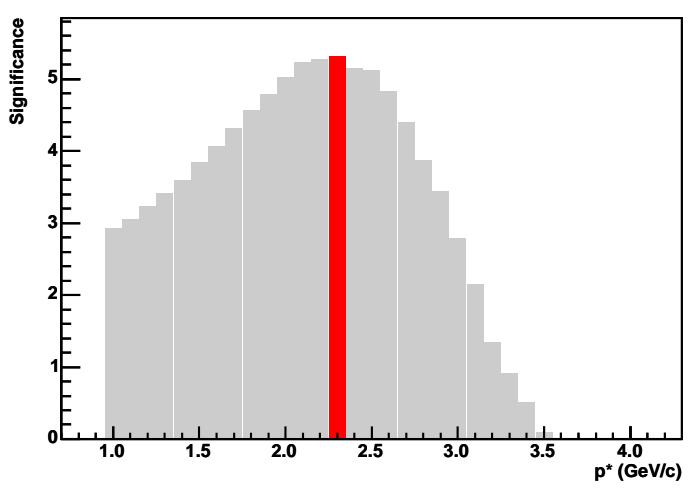

Figure 3.14: The estimated significance versus minimum allowed $p^{*}$ for $\Xi_{c c}^{+}$using the fragmentation-model and side-band data. The red bar indicates the minimum allowed $p^{*}$ resulting in the highest estimated significance.

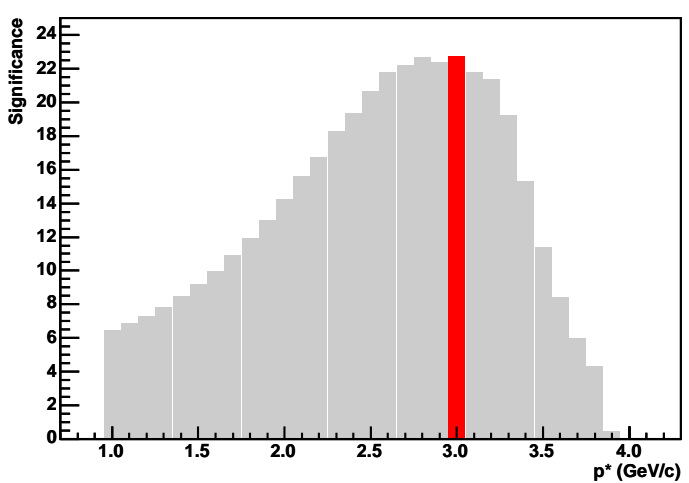

Figure 3.13: The estimated significance versus minimum allowed $p^{*}$ for $\Xi_{c c}^{++}$using the diquarkmodel and side-band data. The red bar indicates the minimum allowed $p^{*}$ resulting in the highest estimated significance.

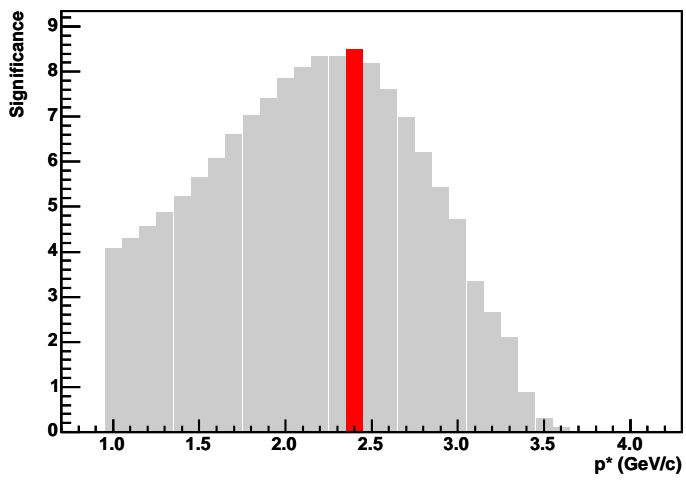

Figure 3.15: The estimated significance versus minimum allowed $p^{*}$ for $\Xi_{c c}^{++}$using the fragmentation-model and side-band data. The red bar indicates the minimum allowed $p^{*}$ resulting in the highest estimated significance.

Table 3.6: Fraction of events with multiple candidates and average number of candidates per event after selection criteria have been applied. All candidates have an invariant-mass difference within the signal region and no $p^{*}$ requirement is made.

\begin{tabular}{llcll}
\hline & $\begin{array}{l}\text { Fraction with Multiple Candidates } \\
\Xi_{c c}^{+}\end{array}$ & $\begin{array}{l}\text { Average Number of Candidates } \\
\Xi_{c c}^{++}\end{array}$ & $\Xi_{c c}^{+}$ & $\Xi_{c c}^{++}$ \\
\hline \hline Fragmentation & $60 \%$ & $46 \%$ & 2.24 & 2.05 \\
\hline Diquark & $52 \%$ & $37 \%$ & 2.00 & 1.80 \\
\hline Data & $58 \%$ & $45 \%$ & 2.27 & 2.11 \\
\hline
\end{tabular}


not used to select the best candidate. All candidates in an event that pass the selection criteria are used in the search.

\subsection{Signal and Background Fitting}

Extended maximum likelihood (ML) fits are performed on the double-charm baryon data in the two-dimensional space of the variables $M_{\Lambda_{c}}=M\left(p K^{-} \pi^{+}\right)$, and $\Delta M=M\left(\left(p K^{-} \pi^{+}\right) K^{-} \pi^{+}\right)-$ $M\left(p K^{-} \pi^{+}\right)$in the $\Xi_{c c}^{+}$search and $\Delta M=M\left(\left(p K^{-} \pi^{+}\right) K^{-} \pi^{+} \pi^{+}\right)-M\left(p K^{-} \pi^{+}\right)$in the $\Xi_{c c}^{++}$search. The $\Xi_{c c}^{+}$and $\Xi_{c c}^{++}$signal-MC candidates are shown with these two variables in Figures 3.16 and 3.17, respectively. The double-charm baryons and $\Lambda_{c}^{+}$baryons in the signal-MC samples are generated with zero natural width, making the signal shape strictly due to measurement resolution. The signals for $\Xi_{c c}^{+}$and $\Xi_{c c}^{++}$are at the intersection of the horizontal and diagonal bands of points. The horizontal bands are candidates that have $M_{\Lambda_{c}}$ corresponding to the $\Lambda_{c}^{+}$mass, but have $\Delta M$ that are broadly distributed, indicating that these candidates are not correctly reconstructed double-charm baryons. Such a distribution is indistinguishable from $\Lambda_{c}^{+}$combinatoric background, where a $\Lambda_{c}^{+}$is correctly reconstructed but the rest of the tracks in the candidate are a random combination of tracks from the rest of the event. This is an example of incorrectly reconstructed signal events creating a background distribution; this type of background distribution is known as self-cross-feed background (SCFB). The diagonal bands of points are candidates that have $\left[M_{\Lambda_{c}}+\Delta M\right]$ corresponding to a doublecharm baryon mass, but have a broad distribution in $M_{\Lambda_{c}}$. This is another example of SCFB; the combination of all five or six tracks are correct to form the double-charm baryoncandidate, but which tracks form the $\Lambda_{c}^{+}$daughter candidate is incorrect. SCFB peaking in $\left[M_{\Lambda_{c}}+\Delta M\right]$ is distinctive from other backgrounds, however, and these events are treated as signal. Data distributions show that the background is smoothly varying in both $\Delta M$ and $M_{\Lambda_{c}}$.

The probability density functions (PDFs) used to fit the $\Xi_{c c}^{+}$and $\Xi_{c c}^{++}$candidates have the same form. First, I describe the background components and the signal components of the PDF in detail. A mathematical description of the PDF then follows.

- The PDF has two background components. One background component is a first-order polynomial in $M_{\Lambda_{c}}$ multiplied by a first-order polynomial in $\Delta M$; this component accounts for candidates that are due to a random combination of charged particles in the event (combinatoric background). The other background component in the PDF accounts for candidates with

a true $\Lambda_{c}^{+}$combined with a random kaon and/or pion(s). This second component is described by a Gaussian in $M_{\Lambda_{c}}$. The amplitude of the Gaussian is allowed to vary linearly in $\Delta M$.

- The PDF also has two signal components. One signal component is a Gaussian in the $\Delta M$ variable multiplied by a Gaussian in $M_{\Lambda_{c}}$. This signal Gaussian in $M_{\Lambda_{c}}$ shares a common width and a common mean with the Gaussian describing the background $\Lambda_{c}^{+}$shape. A second signal component accounts for the SCFB peaking in $\left[M_{\Lambda_{c}}+\Delta M\right]$ and is parameterized by 


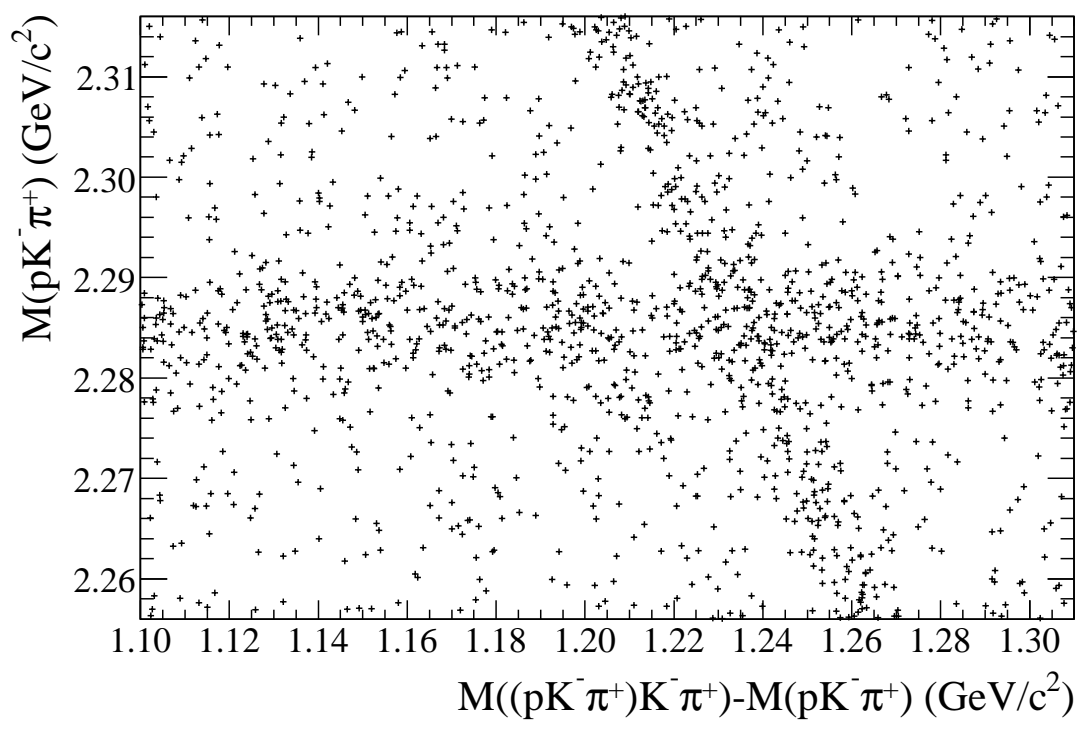

Figure 3.16: Signal-MC $\Xi_{c c}^{+}$in the two-dimensional $\Delta M$ vs. $M_{\Lambda_{c}}$ signal region showing self-cross-feed background from false candidates passing the selection criteria. Misreconstructed $\Xi_{c c}^{+}$candidates with a correctly-reconstructed $\Lambda_{c}^{+}$show up as a horizontal band in the figure. Candidates with a misreconstructed $\Lambda_{c}^{+}$but containing all the correct $\Xi_{c c}^{+}$daughter tracks show up as a diagonal band.

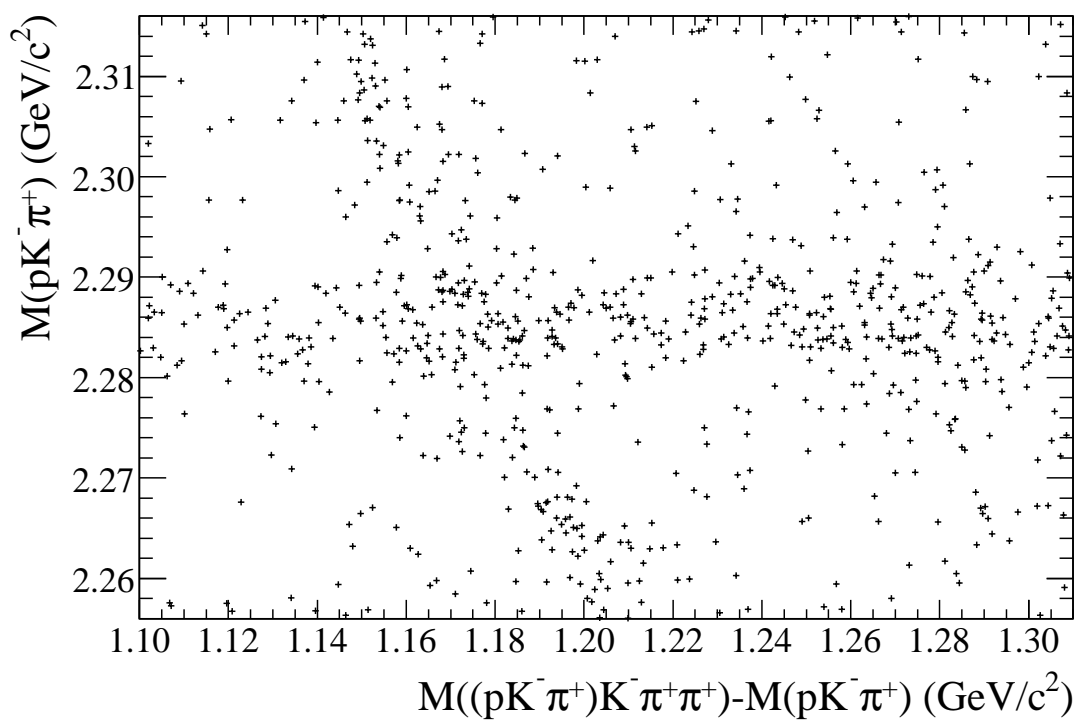

Figure 3.17: Signal-MC $\Xi_{c c}^{++}$in the two-dimensional $\Delta M$ vs. $M_{\Lambda_{c}}$ signal region showing self-crossfeed background from false candidates passing the selection criteria. Misreconstructed $\Xi_{c c}^{++}$candidates with a correctly-reconstructed $\Lambda_{c}^{+}$show up as a horizontal band in the figure. Candidates with a misreconstructed $\Lambda_{c}^{+}$but containing all the correct $\Xi_{c c}^{++}$daughter tracks show up as a diagonal band. 
a Gaussian in $\left[M_{\Lambda_{c}}+\Delta M\right]$; this SCFB Gaussian has a mean that is fixed to be the sum of the signal Gaussian means in $M_{\Lambda_{c}}$ and $\Delta M$. This SCFB is counted as signal because these candidates peak in $\left[M_{\Lambda_{c}}+\Delta M\right]$, are caused by real signal events, and are distinguishable from background.

The full PDF is proportional to

$$
\begin{aligned}
P\left(M_{\Lambda_{c}}, \Delta M\right)=n_{s} \times & (1-f) \times \mathcal{G}\left(M_{\Lambda_{c}} ; \mu_{\Lambda_{c}}, \sigma_{\Lambda_{c}}\right) \times \mathcal{G}\left(\Delta M ; \mu_{s}, \sigma_{s 1}\right) \\
+ & n_{s} \times f \times \mathcal{G}\left(\left[M_{\Lambda_{c}}+\Delta M\right] ;\left[\mu_{\Lambda_{c}}+\mu_{s}\right], \sigma_{s 2}\right) \\
+ & n_{b 1} \times \mathcal{G}\left(M_{\Lambda_{c}} ; \mu_{\Lambda_{c}}, \sigma_{\Lambda_{c}}\right) \times \mathcal{P}\left(\Delta M ; a_{1}\right) \\
& +n_{b 2} \times \mathcal{P}\left(M_{\Lambda_{c}} ; a_{2}\right) \times \mathcal{P}\left(\Delta M ; a_{3}\right)
\end{aligned}
$$

where $\mathcal{G}$ is a Gaussian function and $\mathcal{P}$ is a first-order polynomial. The subscripts $s$ and $b$ indicate whether a parameter pertains to signal or background, and the subscripts 1,2 , and 3 simply enumerate the parameters. The parameter $f$ is the fraction of the signal that is due to SCFB; this fraction is fixed based on the fraction found in the corresponding signal-MC sample. The parameters $\mu_{\Lambda_{c}}$ and $\mu_{s}$ are the means of the $\Lambda_{c}^{+}$Gaussian and the double-charm baryon Gaussian, respectively. The parameters $\sigma_{s 1}$ and $\sigma_{s 2}$ are the Gaussian widths of the double-charm baryon signal in the variables $\Delta M$ and $\left[M_{\Lambda_{c}}+\Delta M\right]$, respectively; the $\sigma_{s 2}$ parameter has a fixed proportionality to $\sigma_{s 1}$, which is determined from the signal-MC samples. The parameters $a_{j}$ are the slope parameters. All signal shape parameters in the PDF are listed in Table 3.7 with the ranges of allowed values in the fit or their fixed values. These allowed ranges define the scope of the search.

The fit to data that I use to search for double-charm baryon signal have a $100 \mathrm{MeV} / c^{2} \Delta M$ range centered on the $10 \mathrm{MeV} / c^{2}$ region allowed for the mean of the signal shape. I fit the data differently, however, in order to determine background parameters to generate TMC background distributions as discussed in Section 3.7. I use $\Delta M$ from $1.05 \mathrm{GeV} / c^{2}$ to $1.36 \mathrm{GeV} / c^{2}$, which includes the signal region as well as the side-band region, to determine background parameters. This range of data and their fit are shown in Figures 3.18 through 3.21 for the four data sets $\left(\Xi_{c c}^{+}\right.$and $\Xi_{c c}^{++}$, with and without $p^{*}$ requirements). The ML fits to these data only have background PDF components. The order of the background component polynomials in the $\Delta M$ dimension are modified to accommodate the larger range: for the $\Xi_{c c}^{+}$candidate distributions with and without $p^{*}$ requirements, the polynomial is second order, for the $\Xi_{c c}^{++}$candidate distribution without the $p^{*}$ requirement, the polynomial is third order, and for the $\Xi_{c c}^{++}$candidate distribution with the $p^{*}$ requirement, the polynomial is first order.

\subsection{Efficiencies}

In order to determine the product of $e^{+} e^{-}$production cross-section and branching fractions, the efficiencies for reconstructing $\Xi_{c c}^{+}, \Xi_{c c}^{++}$, and $\Lambda_{c}^{+}$baryons are estimated using the signal-MC samples. 
Table 3.7: The double-charm baryon signal shape parameters. The ranges given are the minimum and maximum values allowed in the fits; when one value is given that parameter is fixed. SCFB referes to self-cross-feed background.

\begin{tabular}{lcc}
\hline Description & Parameter & Range or Fixed Value \\
\hline \hline Width of the $\Xi_{c c}^{+}$signal Gaussian in $\Delta M$ & $\sigma_{s 1}$ & $(3.0-4.0) \mathrm{MeV} / c^{2}$ \\
\hline Width of the $\Xi_{c c}^{++}$signal Gaussian in $\Delta M$ & $\sigma_{s 1}$ & $(2.5-3.5) \mathrm{MeV} / c^{2}$ \\
\hline Mean of the $\Xi_{c c}^{+}$and $\Xi_{c c}^{++}$signal Gaussian in $\Delta M$ & $\mu_{s}$ & $(1.100-1.310) \mathrm{GeV} / c^{2}$ \\
\hline Fraction of SCFB $\Xi_{c c}^{+}$signal events, $p^{*}>2.3$ & $f$ & $27 \%$ \\
\hline Fraction of SCFB $\Xi_{c c}^{+}$signal events, all $p^{*}$ & $f$ & $28 \%$ \\
\hline Fraction of SCFB $\Xi_{c c}^{++}$signal events, $p^{*}>2.3$ & $f$ & $24 \%$ \\
\hline Fraction of SCFB $\Xi_{c c}^{++}$signal events, all $p^{*}$ & $f$ & $20 \%$ \\
\hline Fraction of SCFB $\Lambda_{c}(2880)^{+}$signal events & $f$ & 2.2 \\
\hline \hline Width of the $\Xi_{c c}^{+} \mathrm{SCFB} / \sigma_{s 1}, p^{*}>2.3$ & $\sigma_{s 2} / \sigma_{s 1}$ & 3.8 \\
\hline Width of the $\Xi_{c c}^{+} \mathrm{SCFB} / \sigma_{s 1}$, all $p^{*}$ & $\sigma_{s 2} / \sigma_{s 1}$ & 2.7 \\
\hline Width of the $\Xi_{c c}^{++} \mathrm{SCFB} / \sigma_{s 1}, p^{*}>2.3$ & $\sigma_{s 2} / \sigma_{s 1}$ & $\sigma_{s 2} / \sigma_{s 1}$ \\
\hline Width of the $\Xi_{c c}^{++} \mathrm{SCFB} / \sigma_{s 1}$, all $p^{*}$ & & \\
\hline
\end{tabular}
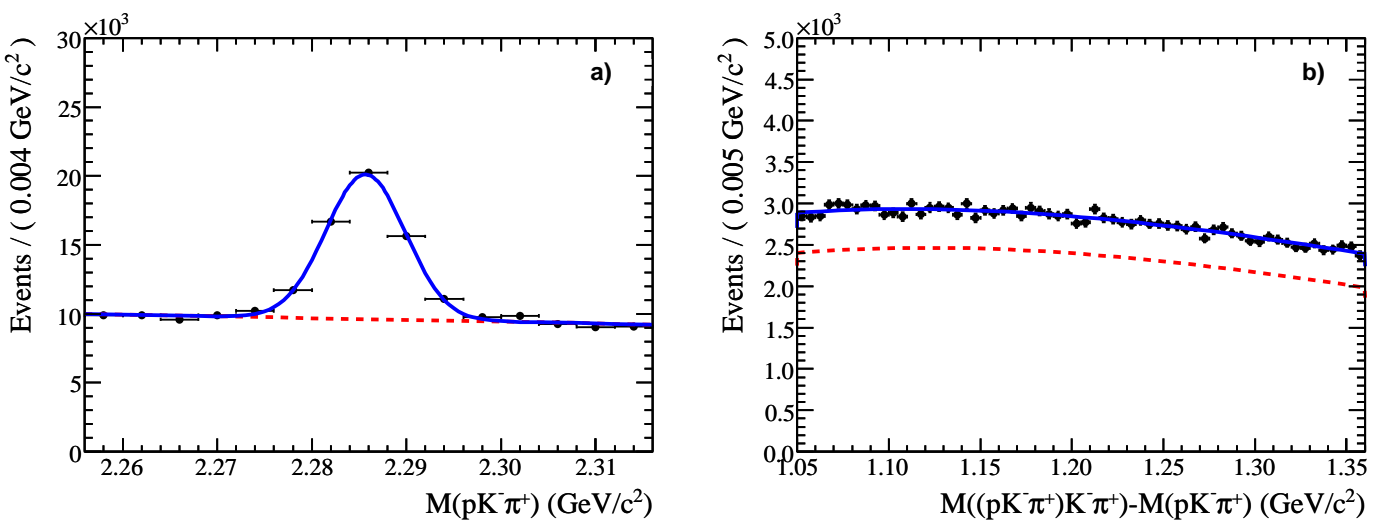

Figure 3.18: Invariant-mass distributions of $\Xi_{c c}^{+}$candidates with no $p^{*}$ requirement. Plot a) shows the $M_{\Lambda_{c}}$ projection and plot b) shows the $\Delta M$ projection. The total background PDF (solid blue curve) and the non- $\Lambda_{c}^{+}$background component (dashed red curve) are shown. The two-dimensional $\chi^{2} /$ n.d.f. goodness-of-fit statistic for this fit is $1013 / 930$ and $P\left(\chi^{2}\right)=0.03$. 

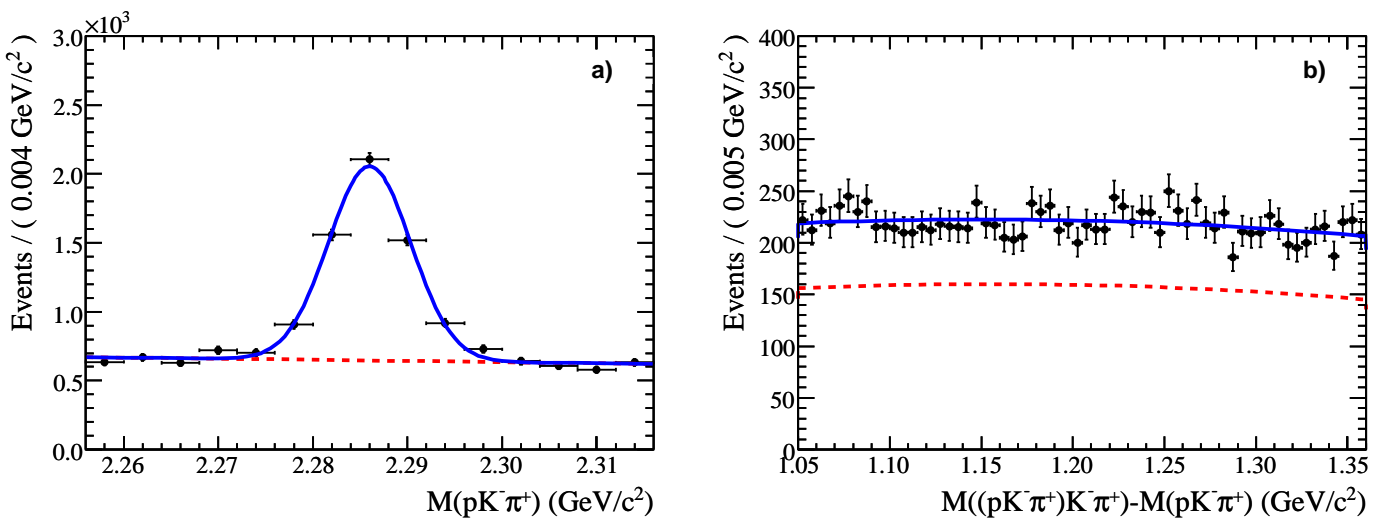

Figure 3.19: Invariant-mass distributions of $\Xi_{c c}^{+}$candidates with $p^{*}>2.3 \mathrm{GeV} / c^{2}$. Plot a) shows the $M_{\Lambda_{c}}$ projection and plot b) shows the $\Delta M$ projection. The total background PDF (solid blue curve) and the non- $\Lambda_{c}^{+}$background component (dashed red curve) are shown. The two-dimensional $\chi^{2} /$ n.d.f. goodness-of-fit statistic for this fit is $942 / 930$ and $P\left(\chi^{2}\right)=0.39$.
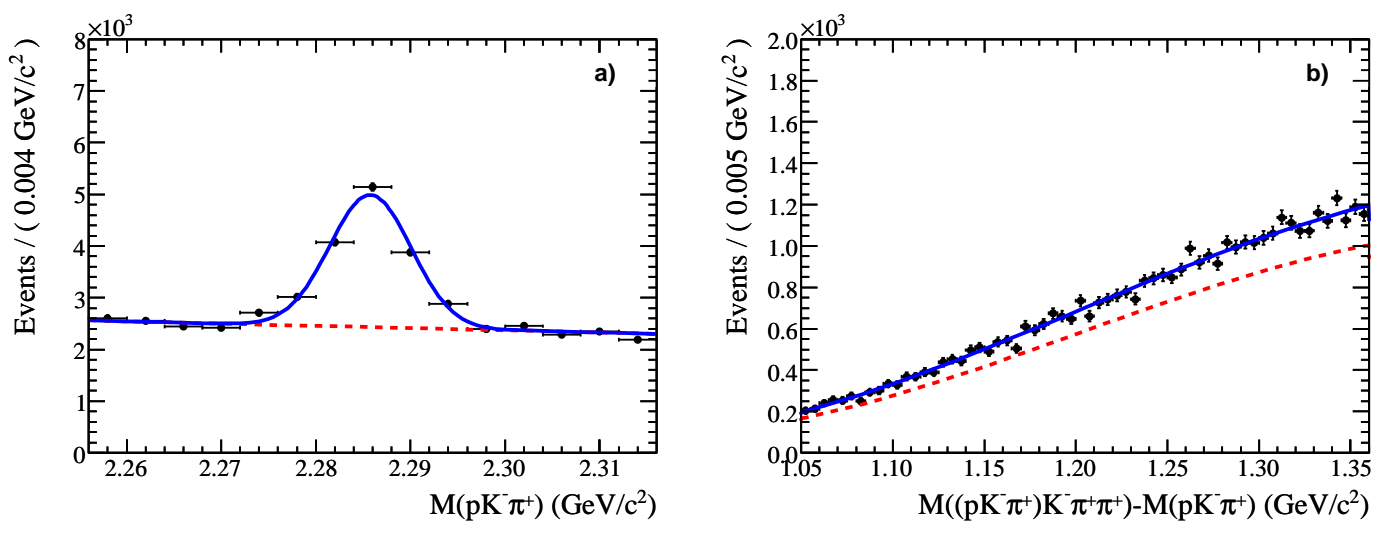

Figure 3.20: Invariant-mass distributions of $\Xi_{c c}^{++}$candidates with no $p^{*}$ requirement. Plot a) shows the $M_{\Lambda_{c}}$ projection and plot b) shows the $\Delta M$ projection. The total background PDF (solid blue curve) and the non- $\Lambda_{c}^{+}$background component (dashed red curve) are shown. The two-dimensional $\chi^{2} /$ n.d.f. goodness-of-fit statistic for this fit is $918 / 930$ and $P\left(\chi^{2}\right)=0.60$. 

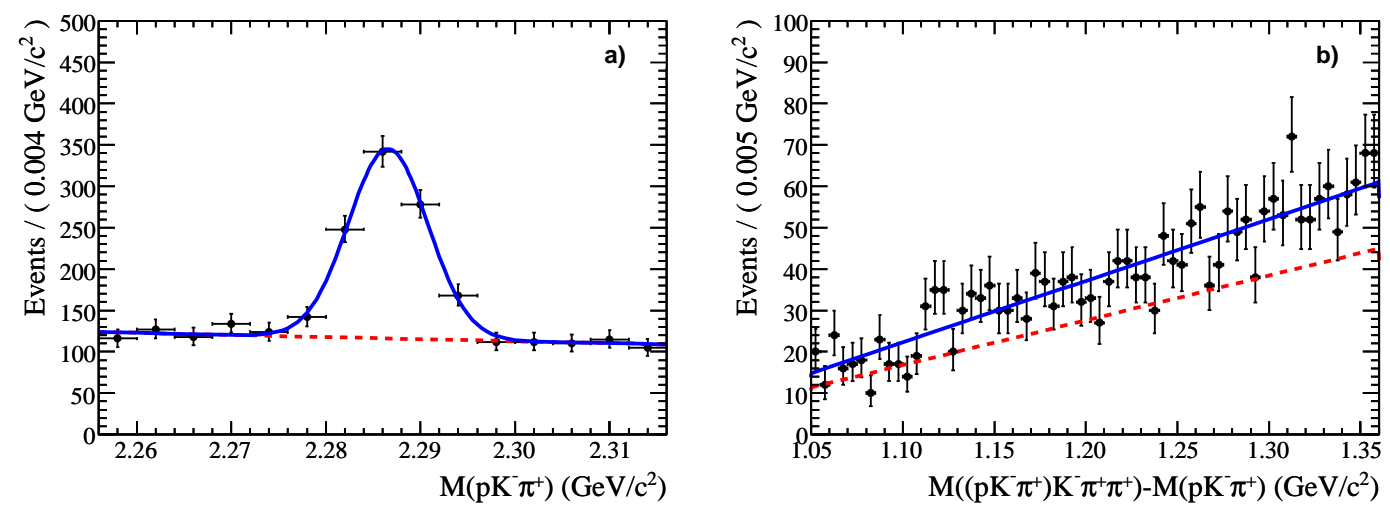

Figure 3.21: Invariant-mass distributions of $\Xi_{c c}^{++}$candidates with $p^{*}>2.3 \mathrm{GeV} / c^{2}$. Plot a) shows the $M_{\Lambda_{c}}$ projection and plot b) shows the $\Delta M$ projection. The total background PDF (solid blue curve) and the non- $\Lambda_{c}^{+}$background component (dashed red curve) are shown. The two-dimensional $\chi^{2} /$ n.d.f. goodness-of-fit statistic for this fit is $924 / 930$ and $P\left(\chi^{2}\right)=0.55$.

Only the fragmentation-model signal-MC samples are used to calculate efficiencies. The candidates in the signal-MC samples have been subjected to the same selection criteria as in data; the $\Lambda_{c}^{+}$ signal-MC is subject to only the selection criteria pertaining to the $\Lambda_{c}^{+}$. The numbers of signal candidates are derived from ML fits. Unbinned fits are used for $\Xi_{c c}^{+}$and $\Xi_{c c}^{++}$efficiencies whereas a binned fit is used for the $\Lambda_{c}^{+}$efficiency. The $\Lambda_{c}^{+}$signal-MC fit is one-dimensional in $M\left(p K^{-} \pi^{+}\right)$, and the PDF has a double-Gaussian signal shape and a linear background shape.

There are differences in tracking and PID efficiencies between signal-MC samples and data. Weights have been calculated for signal-MC track candidates to account for these differences. These weights are included in the ML fits to signal-MC samples. The distributions of weight values for each of the signal-MC samples are narrow, with widths of about 0.1 , and means of about 0.9 . Because the mean weight is less than 1, the estimated errors are slightly over-estimated [35]. Systematic errors due to the weighting of events are as follows: $0.8 \%$ per track (added linearly), $1.0 \%$ per kaon, $1.0 \%$ per pion, and $4.0 \%$ per proton.

Efficiencies are calculated, with and without $p^{*}$ requirements, as the number of signal candidates found with ML fits signal MC, divided by the number of signal-MC candidates generated. Table 3.8 lists the calculated efficiencies with their statistical and systematic errors. Efficiency errors are incorporated into the results as described in Sections3.7.

There are systematic uncertainties due to the dependence of efficiency on the momentum in $e^{+} e^{-}$center-of-mass frame $\left(p^{*}\right)$ and polar angle $(\cos \theta)$ because the true $p^{*}$ and $\cos \theta$ distributions of $\Xi_{c c}^{+}$and $\Xi_{c c}^{++}$are unknown. Changes in these simulated distributions have large effects on the calculated efficiency. I use the fragmentation and diquark models demonstrate how different $p^{*}$ and $\cos \theta$ distributions change the resultant efficiency. As seen in Figures 3.3 through 3.6, these models 
Table 3.8: Efficiencies from fragmentation-model MC samples. The first error is statistical from the fitting procedure, and the second error is systematic from event weighting.

\begin{tabular}{ccr}
\hline$p^{*}(\mathrm{GeV} / c)$ & Baryon & Efficiency $(\%)$ \\
\hline \hline$>2.3$ & $\Xi_{c c}^{+}$ & $10.35 \pm 0.13 \pm 0.51$ \\
& $\Xi_{c c}^{++}$ & $4.21 \pm 0.09 \pm 0.21$ \\
& $\Lambda_{c}^{+}$ & $22.50 \pm 0.06 \pm 1.01$ \\
\hline$>0.0$ & $\Xi_{c c}^{+}$ & $9.74 \pm 0.11 \pm 0.48$ \\
& $\Xi_{c c}^{++}$ & $3.59 \pm 0.08 \pm 0.18$ \\
& $\Lambda_{c}^{+}$ & $22.05 \pm 0.05 \pm 0.99$ \\
\hline
\end{tabular}

Table 3.9: A comparison of the efficiencies as calculated from fragmentation-model MC samples versus diquark-model $\mathrm{MC}$ samples. The errors are statistical only.

\begin{tabular}{crrrr}
\hline$p^{*}(\mathrm{GeV} / c)$ & Diquark $\Xi_{c c}^{+}$ & Diquark $\Xi_{c c}^{++}$ & Frag. $\Xi_{c c}^{+}$ & Frag. $\Xi_{c c}^{++}$ \\
\hline \hline$>2.3$ & $9.7 \pm 0.3$ & $5.3 \pm 0.2$ & $7.2 \pm 0.3$ & $3.5 \pm 0.2$ \\
\hline$>0$ & $9.8 \pm 0.3$ & $5.0 \pm 0.2$ & $6.5 \pm 0.3$ & $3.4 \pm 0.2$ \\
\hline
\end{tabular}

produce significantly different $p^{*}$ and $\cos \theta$ distributions. Table 3.9 shows efficiencies calculated from ML fits to both fragmentation-model and diquark-model MC samples. The diquark-model efficiencies are on average 1.46 times greater than fragmentation-model efficiencies. Because it is difficult to quantify systematic errors of this type, I do not directly incorporate them into the sources of systematic errors. The reader is then left to interpret the difference in efficiency as they see fit.

\section{6 $\quad \Lambda_{c}^{+}$Reference Measurement}

The cross-section times branching fraction, $\sigma\left(e^{+} e^{-} \rightarrow \Lambda_{c}^{+} X\right) \times \mathcal{B}\left(\Lambda_{c}^{+} \rightarrow p K^{-} \pi^{+}\right)$, is measured as a reference value to compare to cross-section times branching fractions for double-charm baryons. The $\Lambda_{c}^{+}$selection criteria used when selecting double-charm baryon candidates are also used when selecting these $\Lambda_{c}^{+}$candidates. Measurements are also done with and without the $p^{*}>2.3 \mathrm{GeV} / c$ requirement on the $\Lambda_{c}^{+}$candidate. Signal-MC events are used to estimate efficiencies for the reconstruction of these $\Lambda_{c}^{+}$events. The efficiency for candidates with and without the minimum $p^{*}$ requirement are $(23 \pm 1) \%$ and $(22 \pm 1) \%$, respectively. An integrated luminosity of only $(21.5 \pm 0.2) \mathrm{fb}^{-1}$ is needed to acheive a fractional statistical error of less than $1 \%$ for the measurement of cross-section times branching fraction because of the large $\Lambda_{c}^{+}$production cross-section and the large $\Lambda_{c}^{+} \rightarrow p K^{-} \pi^{+}$ efficiency. 


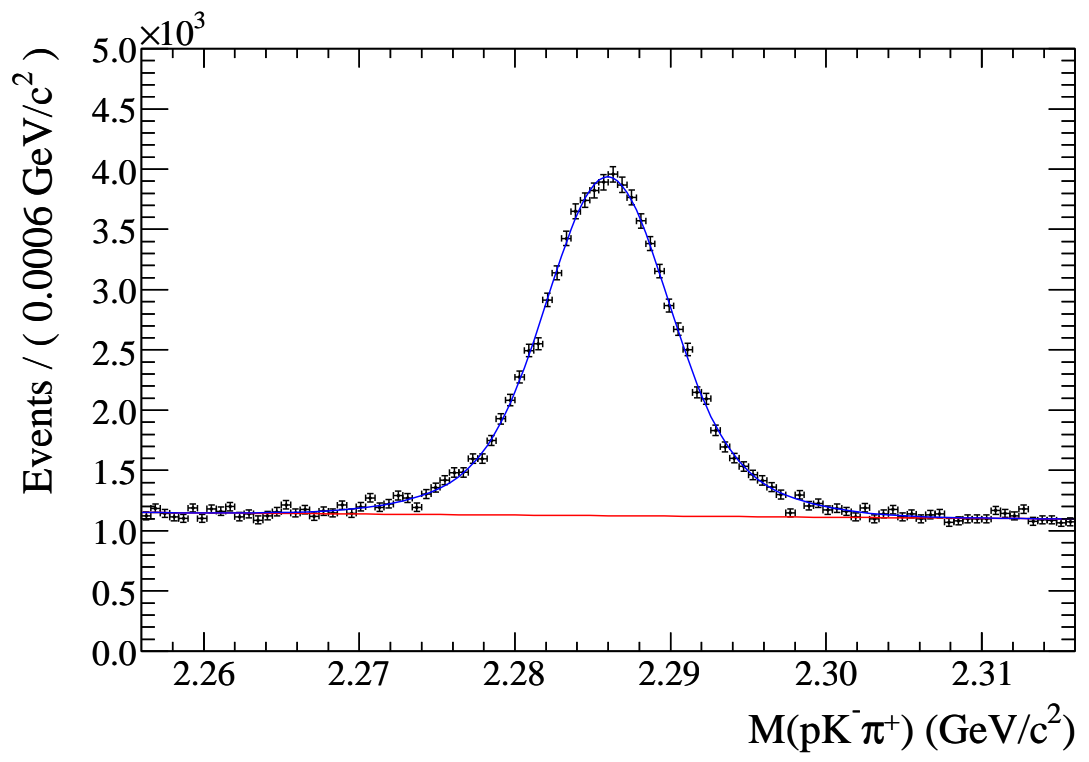

Figure 3.22: One dimensional fit of $M\left(p K^{-} \pi^{+}\right)$invariant-mass distribution with no $p^{*}$ requirement. The blue curve is the total PDF and the red curve is the linear background component of the PDF. The $\chi^{2}$ probability for this fit is $18 \%$.

I perform a binned one-dimensional ML fit on the $M_{\Lambda_{c}}=M\left(p K^{-} \pi^{+}\right)$invariant-mass distribution of $\Lambda_{c}^{+}$candidates. The PDF has a double-Gaussian signal shape and linear background. The results are

$$
\mathcal{S}_{\Lambda_{c}}=\sigma\left(e^{+} e^{-} \rightarrow \Lambda_{c}^{+} X\right) \times \mathcal{B}\left(\Lambda_{c}^{+} \rightarrow p K^{-} \pi^{+}\right)=(8.03 \pm 0.06) \mathrm{pb},
$$

for $\Lambda_{c}^{+}$with $p^{*}>2.3 \mathrm{GeV} / c$, and

$$
\mathcal{S}_{\Lambda_{c}}=\sigma\left(e^{+} e^{-} \rightarrow \Lambda_{c}^{+} X\right) \times \mathcal{B}\left(\Lambda_{c}^{+} \rightarrow p K^{-} \pi^{+}\right)=(10.95 \pm 0.13) \mathrm{pb}
$$

for $\Lambda_{c}^{+}$with no minimum $p^{*}$ requirement. The data and their fits are shown in Figures 3.22 and 3.23. I study systematic errors due to PDF shape by fitting the data with an additional Gaussian shape sharing the same mean as the original two Gaussian shapes. These fits reveal that there are no significant differences in fit results with the additional Gaussian compared to those without. I assign no systematic errors due to fit PDF shape to the $\Lambda_{c}^{+}$results. Systematic uncertainties on luminosity and efficiency are also not included here because they, and other systematic errors, cancel in the ratio with double-charm baryon results. 


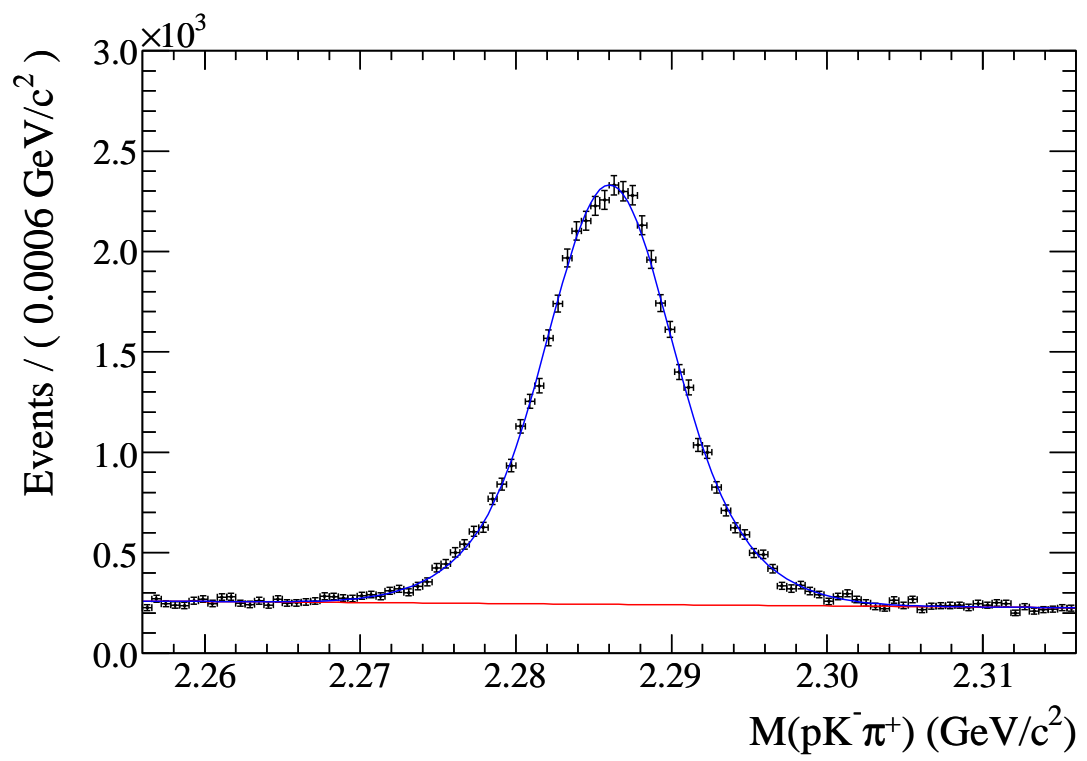

Figure 3.23: One dimensional fit of $M\left(p K^{-} \pi^{+}\right)$invariant-mass distribution with $p^{*}>2.3 \mathrm{GeV} / c$ requirement. The blue curve is the total PDF and the red curve is the linear background component of the PDF. The $\chi^{2}$ probability for this fit is $19 \%$.

\subsection{Search Statistics}

As discussed in Section 3.1, I perform searches for double-charm baryons in a wide parameter space; in particular, for some searches I allow the mean of the signal shape to be anywhere within a $210 \mathrm{MeV} / c^{2}$ range (about 100 times the resolution). When a search has a wide parameter space, the probability of finding a statistical fluctuation in the background distribution can be highly nonGaussian, and this is the case for my searches. In such cases, standard analytical measures of signal significance can be misleading and inaccurate. To calculate the significance of any double-charm baryon signal seen in the data, I use a numerical technique that utilizes toy Monte Carlo (TMC) data samples. I perform a TMC study for each of the eight different searches: $\Xi_{c c}^{+}$with a mass within the $210 \mathrm{MeV} / c^{2}$ invariant-mass search region, $\Xi_{c c}^{+}$with a mass within $5 \mathrm{MeV} / c^{2}$ of $3519 \mathrm{MeV} / c^{2}, \Xi_{c c}^{++}$ with a mass within the $210 \mathrm{MeV} / c^{2}$ invariant-mass search region, $\Xi_{c c}^{++}$with a mass within $5 \mathrm{MeV} / c^{2}$ of $3460 \mathrm{MeV} / c^{2}$, and these four searches again but requiring double-charm baryon candidates to have a $p^{*}$ greater than $2.3 \mathrm{GeV} / c$. For each TMC study I use 1000 simulated invariant-mass distributions generated according to the parameters extracted from the ML fits to the data shown in Figures 3.18, $3.19,3.20$, and 3.21. I use only background components of the PDF in these fits and assume there are no statistically significant peaking signals. Searches for signals are performed on the TMC samples just as they are performed on data as described in Section 3.1. The significance measures, $N / \sigma_{N}$, from these TMC studies are used to create discrete probability density functions (DPDFs). Each 
DPDF can be integrated to yield the probability of finding, in a search of the data, any particular value of significance measure or less, assuming there is no real signal.

There are two qualitatively different DPDFs that result from my TMC studies: one type is for searches withing a $210 \mathrm{MeV} / c^{2}$ mass-difference range, and the other is for searhces within a $10 \mathrm{MeV} / c^{2}$ mass-difference range. The qualitative difference between the DPDFs comes from the selection of the largest of 21 significance measures for each $210 \mathrm{MeV} / c^{2}$ range search verses having only one significance measure for each $10 \mathrm{MeV} / c^{2}$ range search. Figures 3.24 and 3.25 each illustrate a specific example of these two types of DPDFs. I use the DPDF in Figure 3.24 to calculate the significance of a $\Xi_{c c}^{++}$signal within $5 \mathrm{MeV} / c^{2}$ of $3460 \mathrm{MeV} / c^{2}$. I use the DPDF in Figure 3.25 to calculate the significance of a $\Xi_{c c}^{+}$signal within the $210 \mathrm{MeV} / c^{2}$ invariant-mass search region requiring candidates to have $p^{*}>2.3 \mathrm{GeV} / c$. One might naively expect these DPDFs to be Gaussian in shape. This would be true, as shown in Figure 3.26, if all search parameters besides the yield were fixed. Because the mean and width of the signal shape are allowed to vary, however, each fit is more likely to have nonzero yield rather than no yield. Because there are no signals put in the TMC samples, the non-zero values of $N / \sigma_{N}$ are due to statistical fluctuations in the distributions. The DPDFs for the searches within the $210 \mathrm{MeV} / c^{2}$ invariant-mass search region and within a $\pm 5 \mathrm{MeV} / c^{2}$ region are qualitatively different. The DPDFs for searches within a $\pm 5 \mathrm{MeV} / c^{2}$ region are double peaked, with one peak at $N / \sigma_{N}$ just below zero and the other peak at $N / \sigma_{N}$ just above zero. For the searches using the $210 \mathrm{MeV} / c^{2}$ region, only the fit with the largest significance measure for a particular toy distribution is used for the DPDF because only the fit with the largest significance measure is considered as a possible signal in the data. For this reason, the searches using the $210 \mathrm{MeV} / c^{2}$ region have one peak at a positive $N / \sigma_{N}$. Each DPDF is integrated to determine the significance measure at which the data is expected to have a lower value $95 \%$ of the time (assuming that there is no signal). These specific values of significance measure constitute the $1.64 \sigma$ one-sided Gaussian significance level for a signal. Comparing the TMC calculated significance measure needed for a $95 \%$ confidence-level significance to the naive $N / \sigma_{N}=1.64$ required in the fixed parameter example (seen in Figure 3.26), reveals the necessity of the TMC studies. The DPDFs are distinctly non-Gaussian.

Cross-section times branching fractions $(\mathcal{S}=N /(\epsilon \mathrm{L})$, as discussed in Section 3.1) is directly determined from the ML fit using the extended likelihood function

$$
\mathcal{L}=e^{-\left(N-\mathcal{S} f-n_{b}\right)} e^{-\frac{(\mathcal{F}-f)^{2}}{2 \sigma_{\mathcal{F}}}} \prod_{i}^{N} P\left(\vec{x}_{i} ; \mathcal{S}, f, n_{b}, \vec{a}\right)
$$

$N$ is the total number of candidates in the data sample being fit. The measured numbers of signal and background candidates are $\mathcal{S} f=n_{s}$ and $n_{b}$, respectively, The efficiency times luminosity, $(\epsilon \mathrm{L})=\mathcal{F}$, is the conversion factor from cross-section times branching fraction $\mathcal{S}$ to $n_{s}$, and $f$ is the free parameter for this conversion factor. The free parameter $f$ is incorporated into the likelihood function with a $\sigma_{\mathcal{F}}$ Gaussian constraint to have the value $\mathcal{F}$. The Gaussian constraint $\sigma_{\mathcal{F}}$ is the combined statistical 


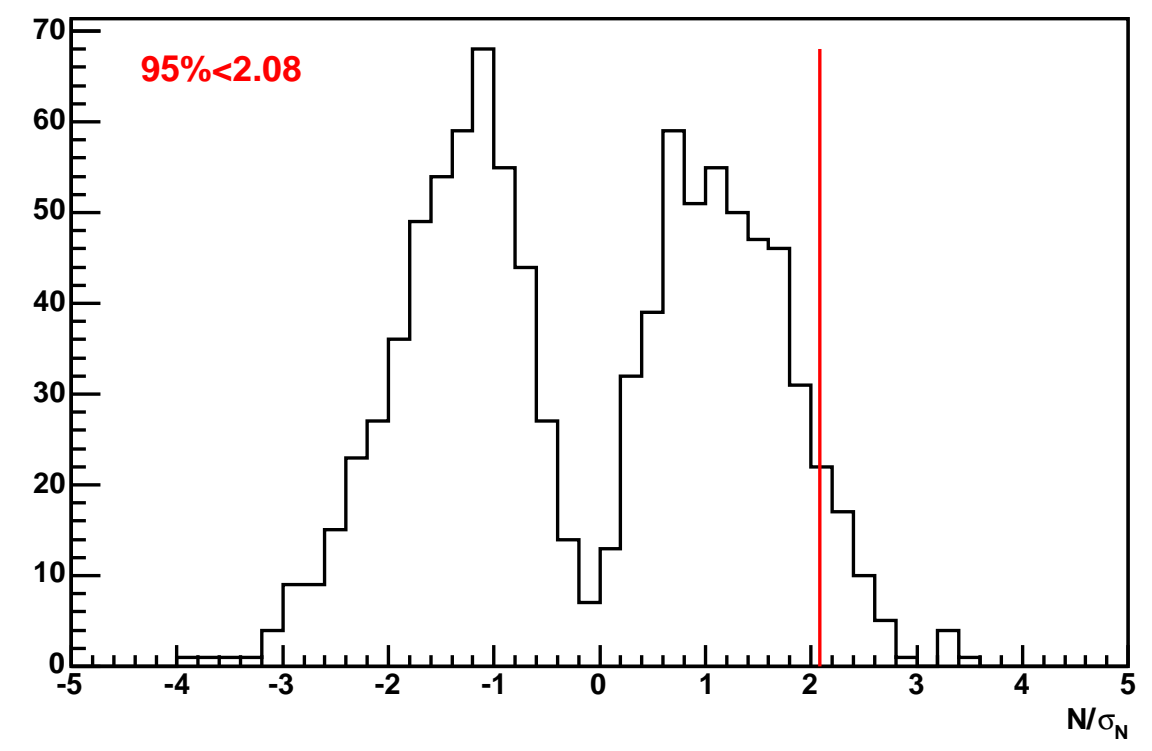

Figure 3.24: Distribution of significance measures from searches performed on 1000 TCM samples for $\Xi_{c c}^{++}$signal within $5 \mathrm{MeV} / c^{2}$ of $3460 \mathrm{MeV} / c^{2}$. This distribution is used as a PDF for calculating the probability of measuring, in a search for $\Xi_{c c}^{++}$in the data, any particular value of significance measure or less, assuming there is no real signal. 95\% of the distribution's area is to the left of the red vertical line at $N / \sigma_{N}=2.08$. 


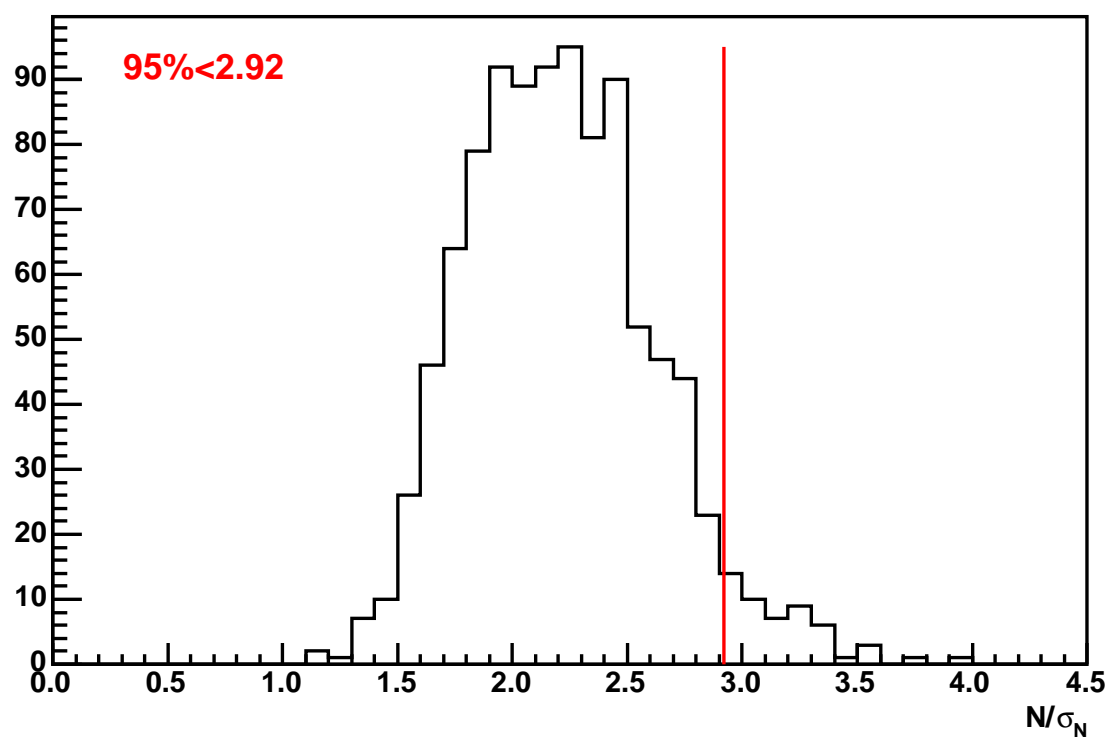

Figure 3.25: Distribution of significance measures from searches performed on 1000 TMC samples for $\Xi_{c c}^{+}$signal within the $210 \mathrm{MeV} / c^{2}$ invariant-mass search region and with $p^{*}>2.3 \mathrm{GeV} / c$. This distribution is used as a PDF for calculating the probability of measuring, in a search for $\Xi_{c c}^{+}$in the data, any particular value of significance measure or less, assuming there is no real signal. $95 \%$ of the histogram area is to the left of the red vertical line at $N / \sigma_{N}=2.92$.

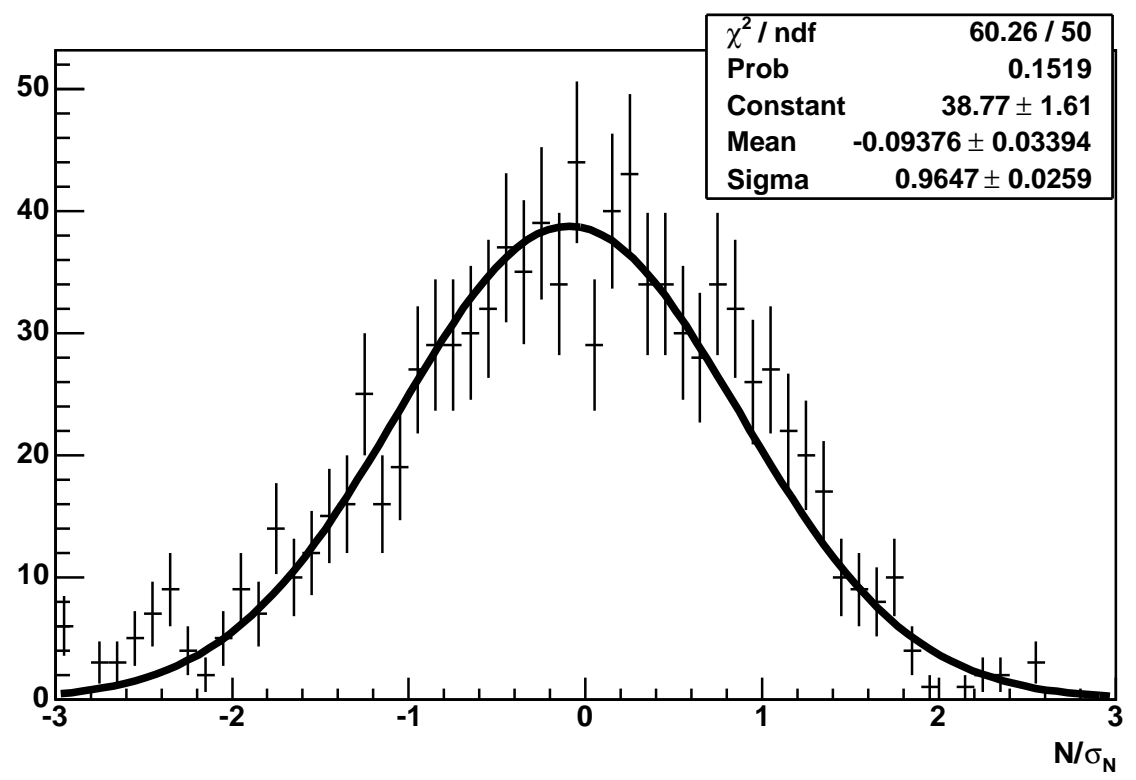

Figure 3.26: Distribution of significance measures from $\Xi_{c c}^{++}$with signal mean and width fixed. The fit shows that this distribution is approximately a unit Gaussian centered at zero as expected. 
and systematic error on $\mathcal{F}$. $P$ is the PDF evaluated for the data point $\vec{x}_{i}$ given the parameters $\mathcal{S}$, $f, n_{b}$, and $\vec{a}$. The parameter $f$ and its Gaussian constraint does not affect the maximization of the likelihood function. When calculating confidence intervals, however, it incorporates systematic errors from the luminosity and efficiency into the result [36]. I modify the conversion factor $\mathcal{F}$ and its error $\sigma_{\mathcal{F}}$ to include the $\sigma\left(e^{+} e^{-} \rightarrow \Lambda_{c}^{+} X\right) \times \mathcal{B}\left(\Lambda_{c}^{+} \rightarrow p K^{-} \pi^{+}\right)$normalization and its error when calculating results with this normalization.

For searches of the data that result in significance measures below $95 \%$ p-value, I calculate $95 \%$ confidence-level upper limits using log-likelihood differences. The technique of log-likelihood difference yields true confidence intervals only assuming Gaussian distributions and large data samples; nevertheless, it is commonly used even when these assumptions do not hold exactly and I use it here. The $95 \%$ confidence-level upper limits are calculated by finding the value of $\mathcal{S}$ where a ML fit with Equation 3.8 of the data results in a maximum log-likelihood 1.353 less than the maximum $\log$-likelihood when $\mathcal{S}$ a free parameter.

\subsection{Results}

For all searches, both for $\Xi_{c c}^{+}$and $\Xi_{c c}^{++}$, with and without the $p^{*}$ requirement, at the masses claimed by SELEX and throughout the $210 \mathrm{MeV} / c^{2}$ search region, no signal is found with a confidence level greater than $95 \%$. Upper limits at $95 \%$ confidence level are set for each search. In addition, each upper limit is also calculated normalized to the appropriate measurement of $\sigma\left(e^{+} e^{-} \rightarrow \Lambda_{c}^{+} X\right) \times$ $\mathcal{B}\left(\Lambda_{c}^{+} \rightarrow p K^{-} \pi^{+}\right)$as described in Sections 3.1 and 3.6.

\subsubsection{Results of $\Xi_{c c}^{+}$Searches}

The searches for $\Xi_{c c}^{+}$within $5 \mathrm{MeV} / c^{2}$ of the $3519 \mathrm{MeV} / c^{2}$ mass claimed by the SELEX Collaboration reveal no significant signal. Figures 3.27 and 3.28 show the $M_{\Lambda_{c}}$ and $\Delta M$ invariant mass projections of the fits to the $\Xi_{c c}^{+}$search region without and with the $p^{*}>2.3 \mathrm{GeV} / c^{2}$ requirement, respectively. With no $p^{*}$ requirement, the yield is $(52 \pm 66)$ candidates. With the $p^{*}$ requirement, the yield is $(-29 \pm 23)$ candidates. There is clearly no significant signal found.

Upper limits are determined as described in Section 3.7. The 95\% confidence-level upper limits for a $\Xi_{c c}^{+}$signal within $5 \mathrm{MeV} / c^{2}$ of $3519 \mathrm{MeV} / c^{2}$ are

$$
\sigma\left(e^{+} e^{-} \rightarrow \Xi_{c c}^{+} X\right) \times \mathcal{B}\left(\Xi_{c c}^{+} \rightarrow \Lambda_{c}^{+} K^{-} \pi^{+}\right) \times \mathcal{B}\left(\Lambda_{c}^{+} \rightarrow p K^{-} \pi^{+}\right)<7.5 \mathrm{fb}
$$

with no minimum $p^{*}$ requirement, and

$$
\sigma\left(e^{+} e^{-} \rightarrow \Xi_{c c}^{+} X\right) \times \mathcal{B}\left(\Xi_{c c}^{+} \rightarrow \Lambda_{c}^{+} K^{-} \pi^{+}\right) \times \mathcal{B}\left(\Lambda_{c}^{+} \rightarrow p K^{-} \pi^{+}\right)<2.2 \mathrm{fb}
$$

with a $p^{*}>2.3 \mathrm{GeV} / c^{2}$ requirement. Normalizing these double-charm baryon results to the $\mathcal{S}_{\Lambda_{c}}=$ 

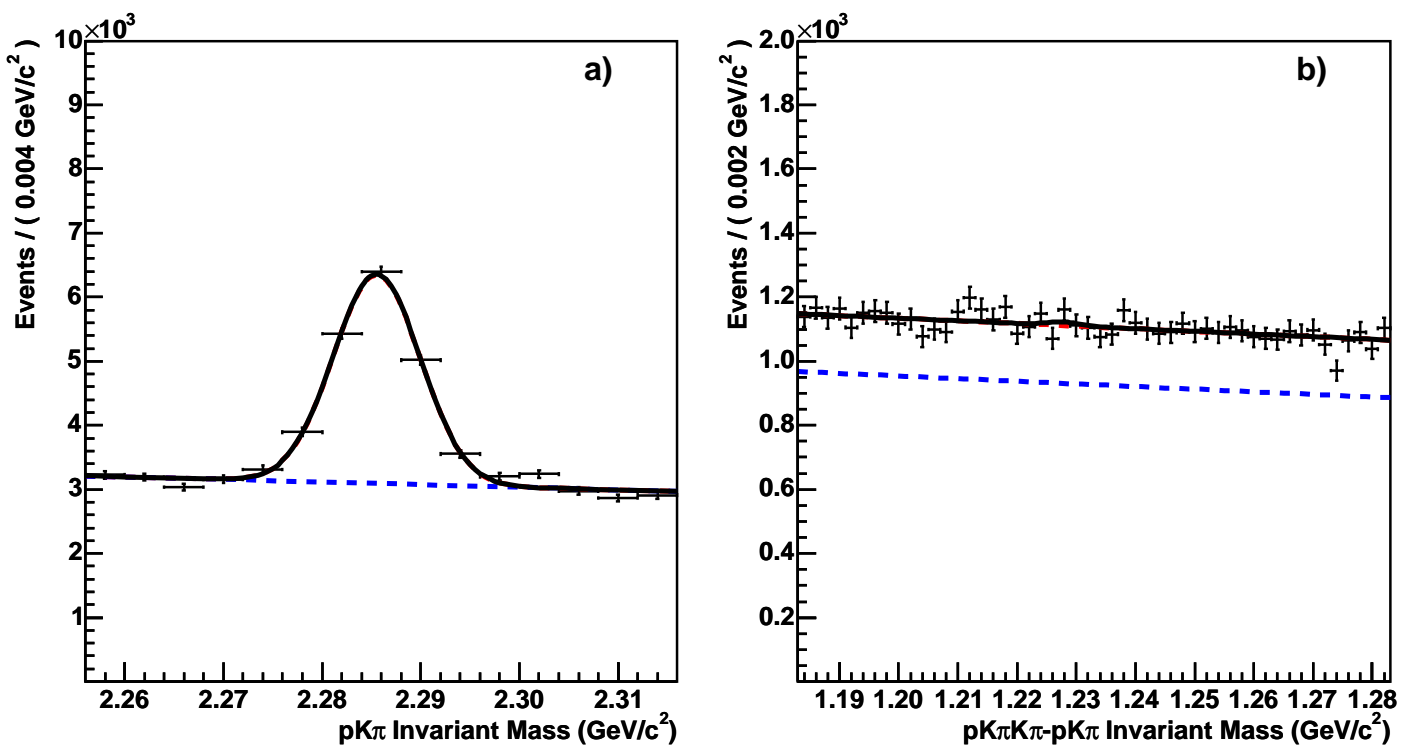

Figure 3.27: a) $M_{\Lambda_{c}}$ and b) $\Delta M$ invariant-mass projections and the fit for $\Xi_{c c}^{+}$signal within $5 \mathrm{MeV} / c^{2}$ of $3519 \mathrm{MeV} / c^{2}$. Data are the points with error bars. The black curve is the PDF. The red dashed curve is the background component of the PDF and the blue dashed curve is the non- $\Lambda_{c}^{+}$background component.
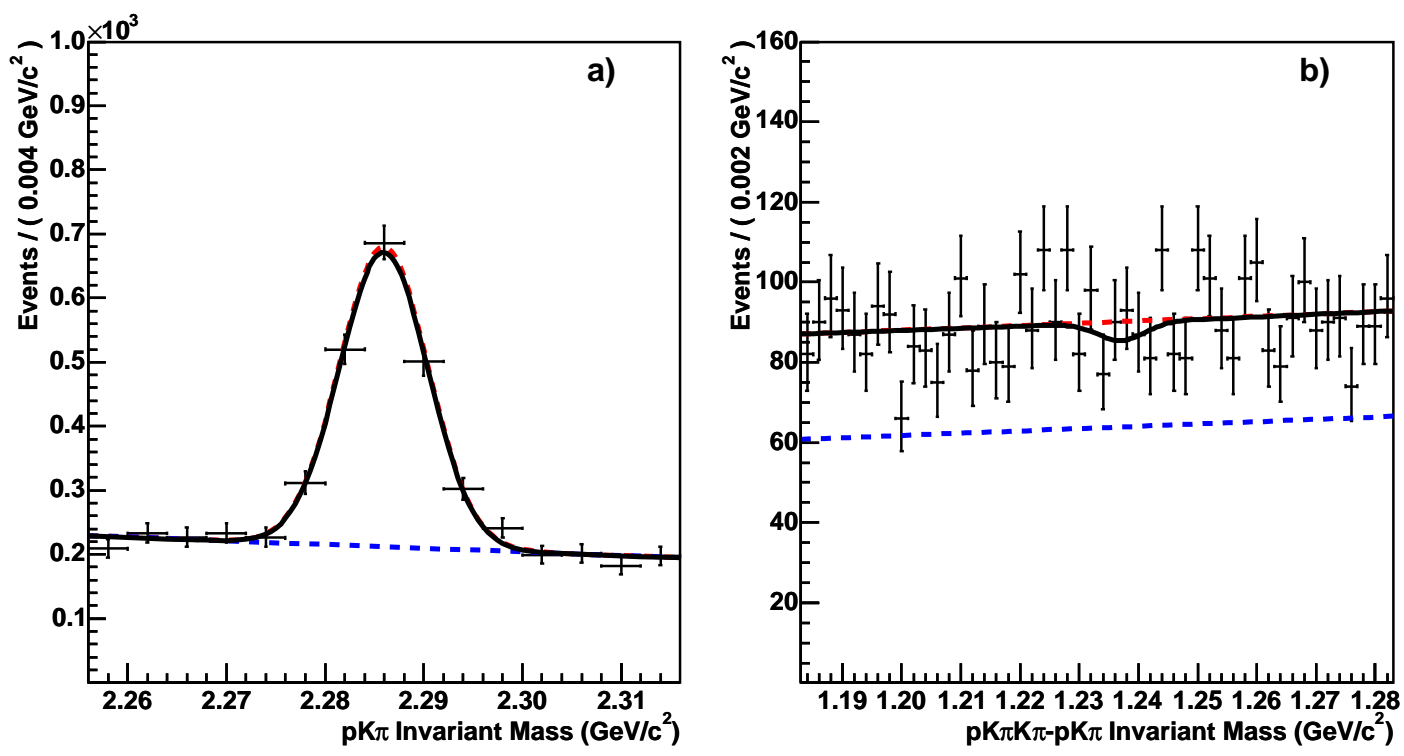

Figure 3.28: a) $M_{\Lambda_{c}}$ and b) $\Delta M$ invariant-mass projections and the fit for $\Xi_{c c}^{+}$signal within $5 \mathrm{MeV} / c^{2}$ of $3519 \mathrm{MeV} / c^{2}$ and $p^{*}>2.3 \mathrm{GeV} / c^{2}$. Data are the points with error bars. The black curve is the PDF. The red dashed curve is the background component of the PDF and the blue dashed curve is the non- $\Lambda_{c}^{+}$background component. 

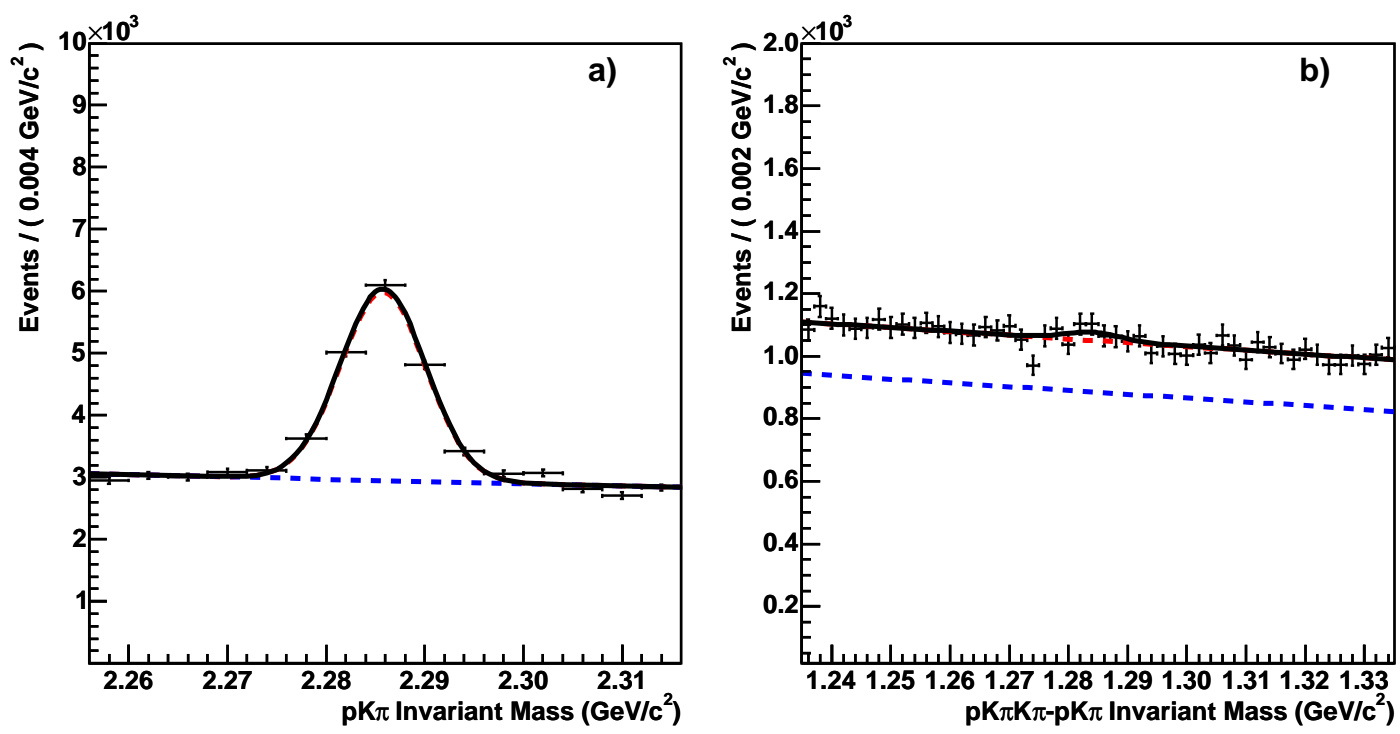

Figure 3.29: a) $M_{\Lambda_{c}}$ and b) $\Delta M$ invariant-mass projections for the most significant fit to the $\Xi_{c c}^{+}$ data. Data are the points with error bars. The black curve is the PDF. The red dashed curve is the background component of the PDF and the blue dashed curve is the non- $\Lambda_{c}^{+}$background component.

$\sigma\left(e^{+} e^{-} \rightarrow \Lambda_{c}^{+} X\right) \times \mathcal{B}\left(\Lambda_{c}^{+} \rightarrow p K^{-} \pi^{+}\right)$results, the $95 \%$ confidence-level upper limits on the ratio for a $\Xi_{c c}^{+}$signal within $5 \mathrm{MeV} / c^{2}$ of $3519 \mathrm{MeV} / c^{2}$ are

$$
\frac{\sigma\left(e^{+} e^{-} \rightarrow \Xi_{c c}^{+} X\right) \times \mathcal{B}\left(\Xi_{c c}^{+} \rightarrow \Lambda_{c}^{+} K^{-} \pi^{+}\right)}{\sigma\left(e^{+} e^{-} \rightarrow \Lambda_{c}^{+} X\right)}<6.9 \times 10^{-4}
$$

with no minimum $p^{*}$ requirement, and

$$
\frac{\sigma\left(e^{+} e^{-} \rightarrow \Xi_{c c}^{+} X\right) \times \mathcal{B}\left(\Xi_{c c}^{+} \rightarrow \Lambda_{c}^{+} K^{-} \pi^{+}\right)}{\sigma\left(e^{+} e^{-} \rightarrow \Lambda_{c}^{+} X\right)}<2.7 \times 10^{-4}
$$

with a $p^{*}>2.3 \mathrm{GeV} / c^{2}$ requirement. The statistical error of the $\Lambda_{c}^{+}$normalizations are incorporated in these upper limits.

The significance measures from 21 fits spanning the $210 \mathrm{MeV} / c^{2} \Xi_{c c}^{+}$search range are listed in Table 3.10. A significance measure of 2.92 would correspond to a $95 \%$ p-value for a signal with $p^{*}>2.3 \mathrm{GeV} / c$, and a significance measure of 3.20 would correspond to a $95 \%$ p-value for a signal with no $p^{*}$ requirement. The fits corresponding to the largest significance measures from data without and with the $p^{*}$ requirement are shown in Figures 3.29 and 3.30, respectively. Both of these signals have a p-value of less than $95 \%$. The $95 \%$ confidence-level upper limits for the $\Xi_{c c}^{+}$results across the search region are also listed in Table 3.10 . 
Table 3.10: The significance measures [SM], the 95\% confidence-level upper limits on cross-section times branching fractions [UL], and the $95 \%$ confidence-level upper limits on cross-section times branching fractions relative to $\sigma\left(e^{+} e^{-} \rightarrow \Lambda_{c}^{+} X\right) \times \mathcal{B}\left(\Lambda_{c}^{+} \rightarrow p K^{-} \pi^{+}\right)\left[\mathrm{UL} / \Lambda_{c}\right]$ for $\Xi_{c c}^{+}$searches.

\begin{tabular}{|c|c|c|c|c|c|c|}
\hline \multirow{2}{*}{$\begin{array}{c}\Delta M \\
\text { Range }\left(\mathrm{GeV} / c^{2}\right)\end{array}$} & \multicolumn{3}{|c|}{$p^{*}>2.3 \mathrm{GeV} / c^{2}$} & \multicolumn{3}{|c|}{$p^{*}>0.0 \mathrm{GeV} / c^{2}$} \\
\hline & $\mathrm{SM}$ & $\mathrm{UL}(\mathrm{fb})$ & $\mathrm{UL} / \Lambda_{c}\left(\times 10^{-4}\right)$ & $\mathrm{SM}$ & $\mathrm{UL}(\mathrm{fb})$ & $\mathrm{UL} / \Lambda_{c}\left(\times 10^{-4}\right)$ \\
\hline $1.10-1.11$ & -0.68 & 1.9 & 2.3 & -2.41 & 3.4 & 3.2 \\
\hline $1.11-1.12$ & +1.23 & 2.6 & 3.3 & +1.49 & 10.3 & 9.4 \\
\hline $1.12-1.13$ & -1.64 & 1.9 & 2.4 & -0.68 & 8.8 & 8.1 \\
\hline $1.13-1.14$ & -1.08 & 1.5 & 1.9 & +1.36 & 9.8 & 9.0 \\
\hline $1.14-1.15$ & -0.96 & 2.1 & 2.7 & -0.43 & 9.6 & 8.8 \\
\hline $1.15-1.16$ & +1.28 & 3.0 & 3.7 & +1.55 & 10.5 & 9.6 \\
\hline $1.16-1.17$ & -1.62 & 1.9 & 2.4 & -1.15 & 8.8 & 8.0 \\
\hline $1.17-1.18$ & -1.69 & 1.3 & 1.6 & -1.80 & 5.2 & 4.8 \\
\hline $1.18-1.19$ & +1.68 & 3.7 & 4.6 & -0.00 & 8.1 & 7.4 \\
\hline $1.19-1.20$ & +2.07 & 3.7 & 4.6 & -0.22 & 8.7 & 8.0 \\
\hline $1.20-1.21$ & -0.81 & 2.7 & 3.4 & -1.27 & 8.9 & 8.2 \\
\hline $1.21-1.22$ & -1.00 & 2.2 & 2.8 & +2.09 & 13.2 & 12.1 \\
\hline $1.22-1.23$ & +0.56 & 2.4 & 3.0 & +1.13 & 11.5 & 10.5 \\
\hline $1.23-1.24$ & -1.38 & 1.6 & 2.0 & -1.29 & 5.3 & 4.8 \\
\hline $1.24-1.25$ & -0.87 & 1.9 & 2.3 & -0.78 & 4.0 & 3.6 \\
\hline $1.25-1.26$ & +2.78 & 4.4 & 5.6 & +1.68 & 10.2 & 9.4 \\
\hline $1.26-1.27$ & +2.09 & 3.7 & 4.7 & -1.21 & 6.7 & 6.2 \\
\hline $1.27-1.28$ & +0.63 & 2.4 & 3.1 & +1.53 & 11.7 & 10.8 \\
\hline $1.28-1.29$ & +1.20 & 2.4 & 3.0 & +2.36 & 14.5 & 13.3 \\
\hline $1.29-1.30$ & -1.55 & 1.2 & 1.4 & +1.68 & 12.0 & 11.0 \\
\hline $1.30-1.31$ & +1.42 & 2.8 & 3.5 & -1.65 & 3.6 & 3.3 \\
\hline
\end{tabular}



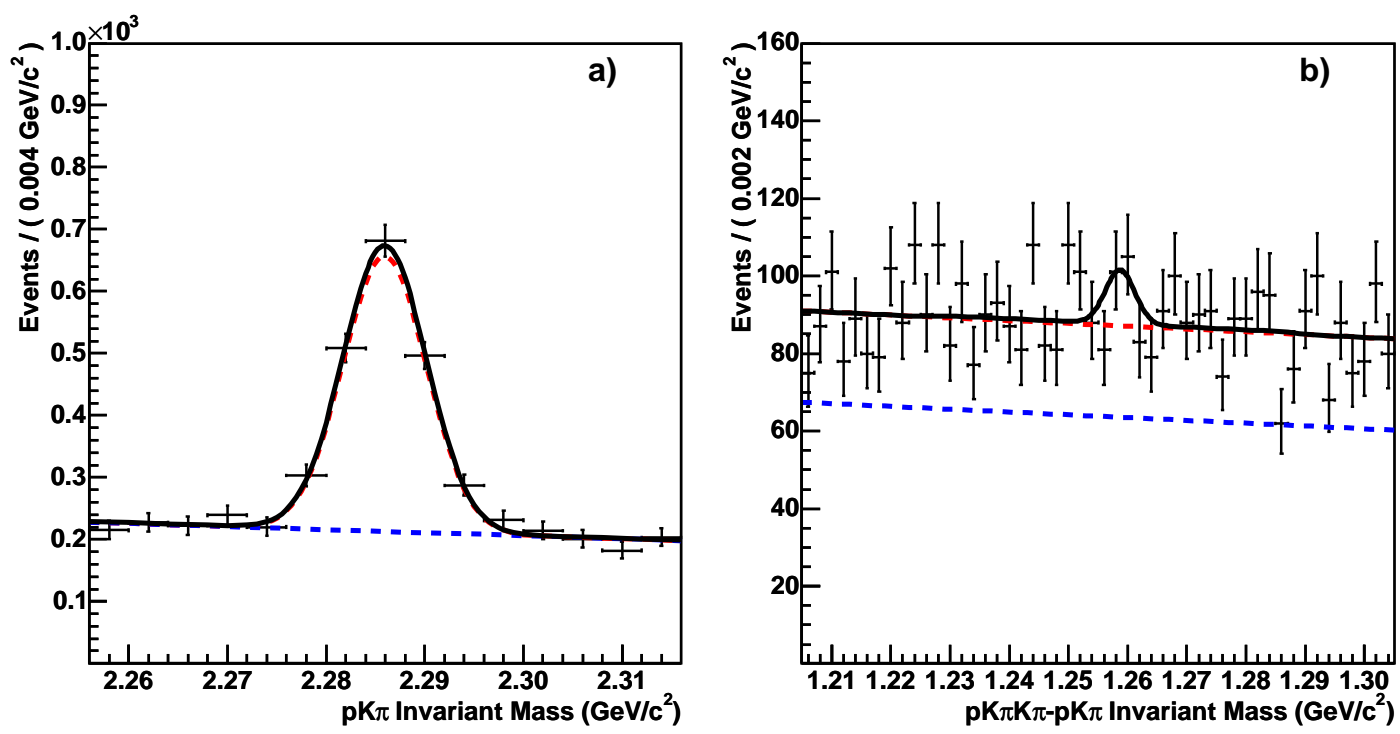

Figure 3.30: a) $M_{\Lambda_{c}}$ and b) $\Delta M$ b) invariant-mass projections for the most significant fit to the $\Xi_{c c}^{+}$ data with $p^{*}>2.3 \mathrm{GeV} / c^{2}$. Data are the points with error bars. The black curve is the PDF. The red dashed curve is the background component of the PDF and the blue dashed curve is the non- $\Lambda_{c}^{+}$ background component.

\subsubsection{Results of $\Xi_{c c}^{++}$Searches}

The searches for $\Xi_{c c}^{++}$within $5 \mathrm{MeV} / c^{2}$ of the $3.460 \mathrm{GeV} / c^{2}$ mass claimed by the SELEX Collaboration reveal no significant signal. Figures 3.31 and 3.32 show the $M_{\Lambda_{c}}$ and $\Delta M$ invariant mass projections of the fits to the $\Xi_{c c}^{++}$search region without and with the $p^{*}>2.3 \mathrm{GeV} / c^{2}$ requirement, respectively. With no $p^{*}$ requirement, the yield is $-40 \pm 22$ candidates. With the $p^{*}$ requirement, the yield is $13 \pm 10$ candidates. There is clearly no significant signal found.

The $95 \%$ confidence-level upper limits for a $\Xi_{c c}^{++}$signal within $5 \mathrm{MeV} / c^{2}$ of $3460 \mathrm{MeV} / c^{2}$ are

$$
\sigma\left(e^{+} e^{-} \rightarrow \Xi_{c c}^{++} X\right) \times \mathcal{B}\left(\Xi_{c c}^{++} \rightarrow \Lambda_{c}^{+} K^{-} \pi^{+} \pi^{+}\right) \times \mathcal{B}\left(\Lambda_{c}^{+} \rightarrow p K^{-} \pi^{+}\right)<11 \mathrm{fb}
$$

with no minimum $p^{*}$ requirement, and

$$
\sigma\left(e^{+} e^{-} \rightarrow \Xi_{c c}^{++} X\right) \times \mathcal{B}\left(\Xi_{c c}^{++} \rightarrow \Lambda_{c}^{+} K^{-} \pi^{+} \pi^{+}\right) \times \mathcal{B}\left(\Lambda_{c}^{+} \rightarrow p K^{-} \pi^{+}\right)<3.2 \mathrm{fb}
$$

with a $p^{*}>2.3 \mathrm{GeV} / c^{2}$ requirement. Normalizing these double-charm baryon results to the $\mathcal{S}_{\Lambda_{c}}=$ $\sigma\left(e^{+} e^{-} \rightarrow \Lambda_{c}^{+} X\right) \times \mathcal{B}\left(\Lambda_{c}^{+} \rightarrow p K^{-} \pi^{+}\right)$results, the $95 \%$ confidence-level upper limits on the ratio for 

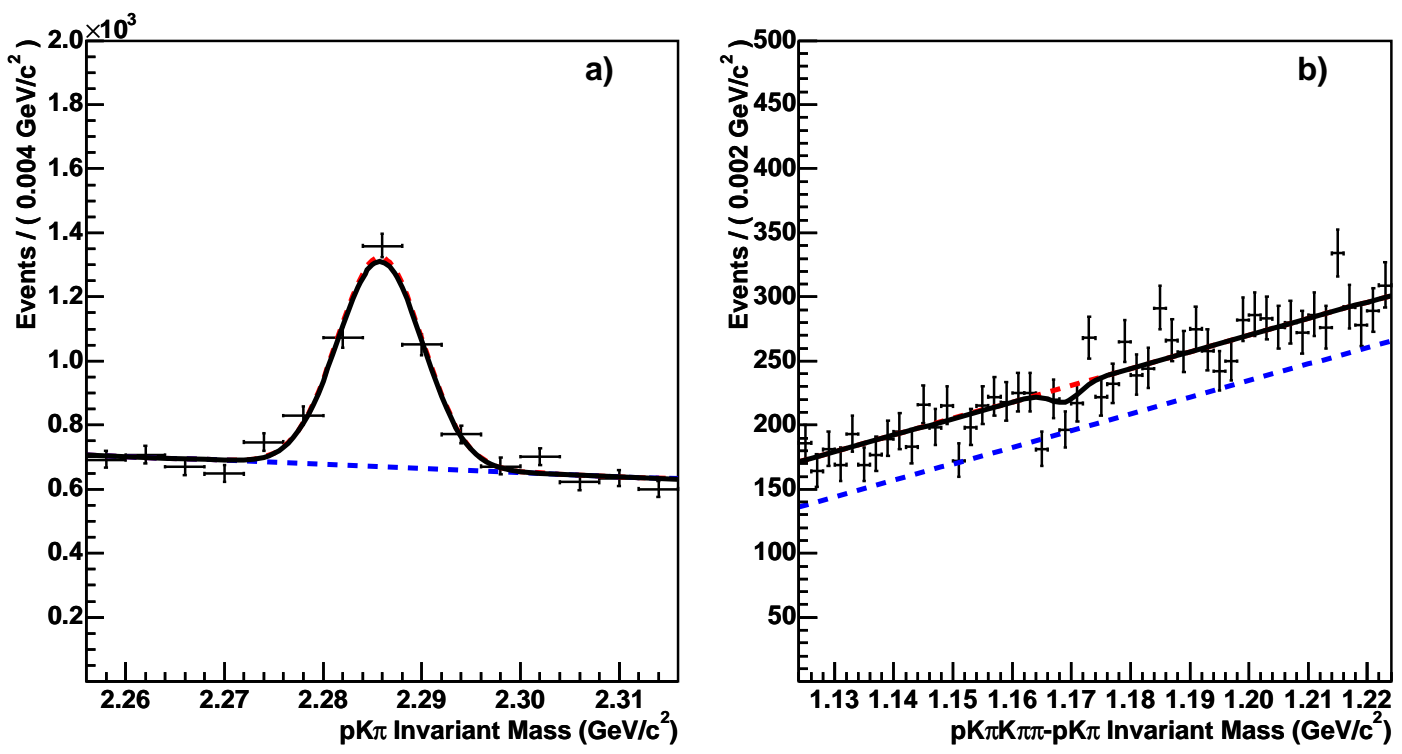

Figure 3.31: a) $M_{\Lambda_{c}}$ and b) $\Delta M$ invariant-mass projections and the fit for $\Xi_{c c}^{++}$signal within $5 \mathrm{MeV} / c^{2}$ of $3460 \mathrm{MeV} / c^{2}$. Data are the points with error bars. The black curve is the PDF. The red dashed curve is the background component of the PDF and the blue dashed curve is the non- $\Lambda_{c}^{+}$ background component.

a $\Xi_{c c}^{++}$signal within $5 \mathrm{MeV} / c^{2}$ of $3460 \mathrm{MeV} / c^{2}$ are

$$
\frac{\sigma\left(e^{+} e^{-} \rightarrow \Xi_{c c}^{++} X\right) \times \mathcal{B}\left(\Xi_{c c}^{++} \rightarrow \Lambda_{c}^{+} K^{-} \pi^{+} \pi^{+}\right)}{\sigma\left(e^{+} e^{-} \rightarrow \Lambda_{c}^{+} X\right)}<10 \times 10^{-4},
$$

with no minimum $p^{*}$ requirement, and

$$
\frac{\sigma\left(e^{+} e^{-} \rightarrow \Xi_{c c}^{++} X\right) \times \mathcal{B}\left(\Xi_{c c}^{++} \rightarrow \Lambda_{c}^{+} K^{-} \pi^{+} \pi^{+}\right)}{\sigma\left(e^{+} e^{-} \rightarrow \Lambda_{c}^{+} X\right)}=4.0 \times 10^{-4},
$$

with a $p^{*}>2.3 \mathrm{GeV} / c^{2}$ requirement. The statistical errors on the $\Lambda_{c}^{+}$normalizations are incorporated in these upper limits.

The significance measures from 21 fits spanning the $\Xi_{c c}^{++}$search range are listed in Table 3.11. A significance measure of 2.75 would correspond to a $95 \%$ p-value for a signal with $p^{*}>2.3 \mathrm{GeV} / c$, and a significance measure of 3.05 would correspond to a $95 \%$ p-value for a signal with no $p^{*}$ requirement. The fits corresponding to the largest significance measures from data without and with the $p^{*}$ requirement are shown in Figures 3.33 and 3.34, respectively. Both of these signals have a significance of less than $95 \%$. The $95 \%$ confidence-level upper limits for the $\Xi_{c c}^{++}$results across the search region are also listed in Table 3.11 . 

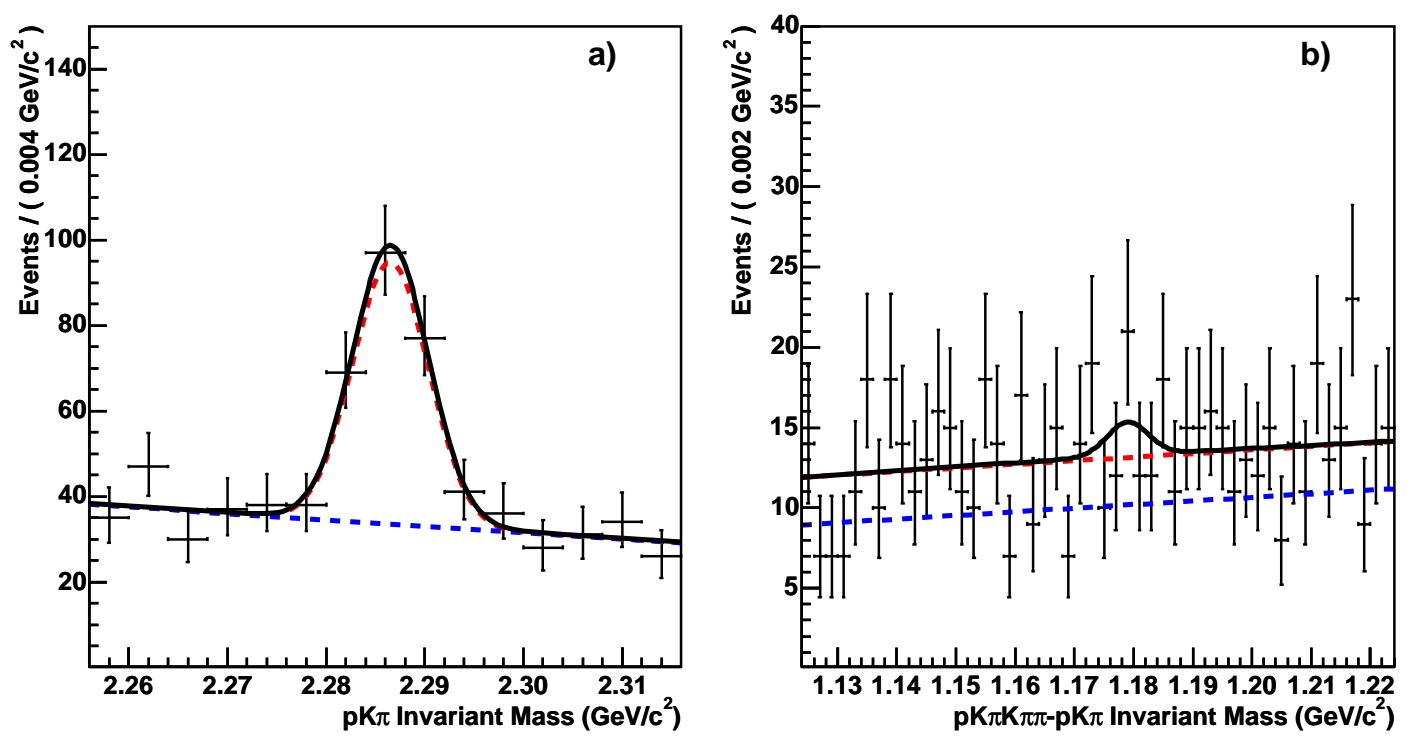

Figure 3.32: a) $M_{\Lambda_{c}}$ and b) $\Delta M$ invariant-mass projections and the fit for $\Xi_{c c}^{++}$signal within $5 \mathrm{MeV} / c^{2}$ of $3460 \mathrm{MeV} / c^{2}$ and $p^{*}>2.3 \mathrm{GeV} / c^{2}$. Data are the points with error bars. The black curve is the PDF. The red dashed curve is the background component of the PDF and the blue dashed curve is the non- $\Lambda_{c}^{+}$background component.
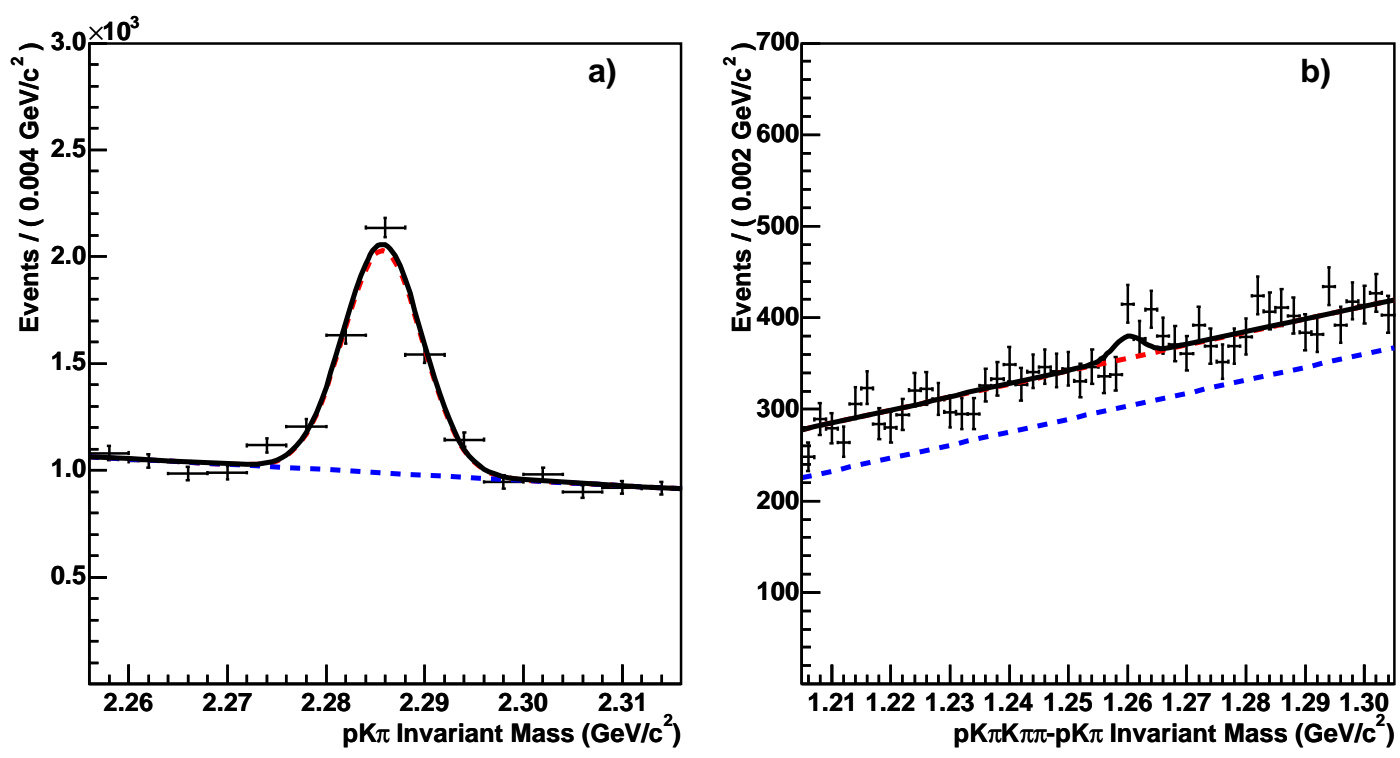

Figure 3.33: a) $M_{\Lambda_{c}}$ and b) $\Delta M$ invariant-mass projections for the most significant fit to the $\Xi_{c c}^{++}$ data. Data are the points with error bars. The black curve is the PDF. The red dashed curve is the background component of the PDF and the blue dashed curve is the non- $\Lambda_{c}^{+}$background component. 
Table 3.11: The significance measures [SM], the 95\% confidence-level upper limits on cross-section times branching fractions [UL], and the $95 \%$ confidence-level upper limits on cross-section times branching fractions relative to $\sigma\left(e^{+} e^{-} \rightarrow \Lambda_{c}^{+} X\right) \times \mathcal{B}\left(\Lambda_{c}^{+} \rightarrow p K^{-} \pi^{+}\right)\left[\mathrm{UL} / \Lambda_{c}\right]$ for $\Xi_{c c}^{++}$searches.

\begin{tabular}{|c|c|c|c|c|c|c|}
\hline \multirow{2}{*}{$\begin{array}{c}\Delta M \\
\text { Range }\left(\mathrm{GeV} / c^{2}\right)\end{array}$} & \multicolumn{3}{|c|}{$p^{*}>2.3 \mathrm{GeV} / c^{2}$} & \multicolumn{3}{|c|}{$p^{*}>0.0 \mathrm{GeV} / c^{2}$} \\
\hline & $\mathrm{SM}$ & $\mathrm{UL}(\mathrm{fb})$ & $\mathrm{UL} / \Lambda_{c}\left(\times 10^{-4}\right)$ & $\mathrm{SM}$ & $\mathrm{UL}(\mathrm{fb})$ & $\mathrm{UL} / \Lambda_{c}\left(\times 10^{-4}\right)$ \\
\hline $1.10-1.11$ & -3.07 & 1.1 & 1.4 & -1.57 & 5.6 & 5.2 \\
\hline $1.11-1.12$ & +1.91 & 2.9 & 3.7 & +0.92 & 8.3 & 7.6 \\
\hline $1.12-1.13$ & -1.75 & 2.6 & 3.3 & -1.13 & 3.9 & 3.5 \\
\hline $1.13-1.14$ & -2.10 & 2.6 & 3.3 & -1.23 & 8.1 & 7.5 \\
\hline $1.14-1.15$ & +1.68 & 3.2 & 4.0 & +1.67 & 11.0 & 10.1 \\
\hline $1.15-1.16$ & +1.02 & 2.9 & 3.6 & +0.98 & 9.2 & 8.4 \\
\hline $1.16-1.17$ & +0.60 & 2.3 & 2.8 & +0.67 & 8.1 & 7.4 \\
\hline $1.17-1.18$ & +1.22 & 3.1 & 3.9 & +0.38 & 10.6 & 9.7 \\
\hline $1.18-1.19$ & +1.01 & 2.9 & 3.6 & +1.44 & 13.1 & 10.7 \\
\hline $1.19-1.20$ & -1.60 & 1.3 & 1.6 & -0.99 & 8.6 & 7.9 \\
\hline $1.20-1.21$ & -1.53 & 1.2 & 1.4 & +0.37 & 8.0 & 7.3 \\
\hline $1.21-1.22$ & +1.06 & 2.2 & 2.7 & +1.49 & 12.5 & 11.4 \\
\hline $1.22-1.23$ & -0.88 & 2.2 & 2.8 & -1.57 & 6.6 & 6.1 \\
\hline $1.23-1.24$ & -3.44 & 1.0 & 1.3 & +0.68 & 11.4 & 10.4 \\
\hline $1.24-1.25$ & +0.74 & 2.2 & 2.7 & -0.28 & 12.3 & 11.3 \\
\hline $1.25-1.26$ & +1.92 & 3.9 & 4.9 & +2.05 & 23.9 & 21.9 \\
\hline $1.26-1.27$ & +1.39 & 3.3 & 4.2 & +2.53 & 20.2 & 18.5 \\
\hline $1.27-1.28$ & -1.15 & 2.2 & 2.8 & +0.53 & 10.8 & 9.9 \\
\hline $1.28-1.29$ & +0.74 & 2.4 & 3.0 & -1.26 & 9.8 & 9.0 \\
\hline $1.29-1.30$ & +0.95 & 3.5 & 4.4 & +0.67 & 11.6 & 10.9 \\
\hline $1.30-1.31$ & +1.14 & 5.5 & 6.9 & +1.95 & 16.4 & 20.0 \\
\hline
\end{tabular}



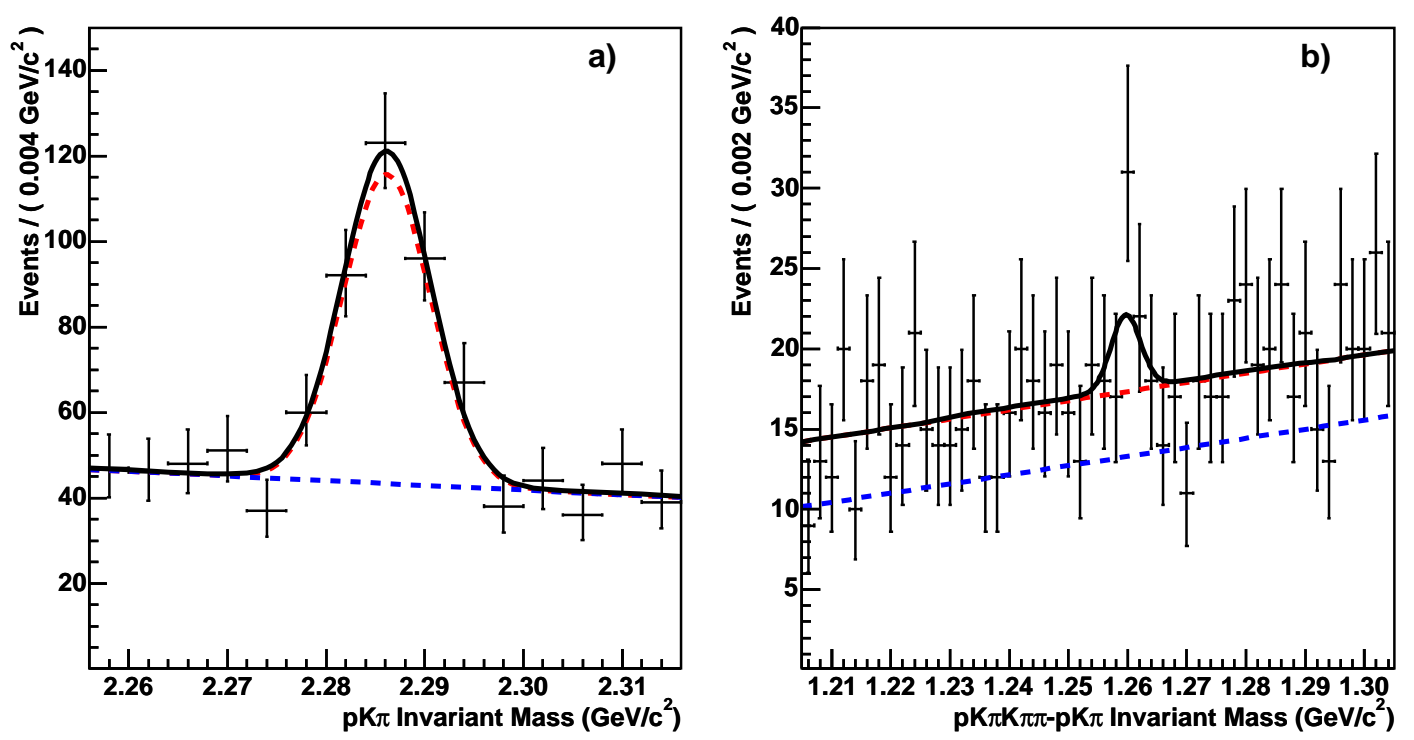

Figure 3.34: a) $M_{\Lambda_{c}}$ and b) $\Delta M$ invariant-mass projections for the most significant fit to the $\Xi_{c c}^{++}$ data with $p^{*}>2.3 \mathrm{GeV} / c^{2}$. Data are the points with error bars. The black curve is the PDF. The red dashed curve is the background component of the PDF and the blue dashed curve is the non- $\Lambda_{c}^{+}$ background component.

\subsection{Conclusions}

My search for the double-charm baryons $\Xi_{c c}^{+}$and $\Xi_{c c}^{++}$reveals no statistically significant signals for such states being produced in $e^{+} e^{-}$collisions at about $10.6 \mathrm{GeV}$. In the absence of signal, I determine several upper limits for possible types of $\Xi_{c c}^{+}$and $\Xi_{c c}^{++}$baryons. I determine $95 \%$ confidence level upper limits for $\Xi_{c c}^{+}$and $\Xi_{c c}^{++}$baryons having masses within $5 \mathrm{MeV} / c^{2}$ of their masses as reported by the SELEX Collaboration; Broadening the allowed parameters for the double-charm baryons, I determine $95 \%$ confidence level upper limits for $\Xi_{c c}^{+}$and $\Xi_{c c}^{++}$baryons with masses within a $210 \mathrm{MeV} / c^{2}$ invariant-mass range from about $3390 \mathrm{MeV} / c^{2}$ to about $3600 \mathrm{MeV} / c^{2}$. The searches within the wider invariant-mass range are less sensitive to finding double-charm baryon signals as can be seen by their higher upper limits on cross-section times branching fractions, $\mathcal{S}$. I also perform all searches with and without a minimum $p^{*}$ requirement of $2.3 \mathrm{GeV} / c$ on the double-charm baryon candidate. The searches with the $p^{*}$ requirement have less background and lower upper limits, but they assume that the double-charm baryons are produced with higher $p^{*}$ distributions than the background. The searches without the $p^{*}$ requirement do not have this assumption and are more readily related to theoretical calculations, but their upper limits on $\mathcal{S}$ are less constraining. I summarize all upper limits in Table 3.12. The upper limits for the $210 \mathrm{MeV} / c^{2}$ wide invariant-mass range in Table 3.12 are the highest limits from Tables 3.10 and 3.11. Other BABAR Collaboration 
Table 3.12: The 95\% confidence-level upper limits on double-charm baryon cross-section times branching fractions $\mathcal{S}_{\Xi_{c c}}$, and the $95 \%$ confidence-level upper limits on cross-section times branching fractions relative to $\mathcal{S}_{\Lambda_{c}}=\sigma\left(e^{+} e^{-} \rightarrow \Lambda_{c}^{+} X\right) \times \mathcal{B}\left(\Lambda_{c}^{+} \rightarrow p K^{-} \pi^{+}\right)$.

\begin{tabular}{ccccc}
\hline & $\mathcal{S}_{\Xi_{c c}^{+}}$ & $\mathcal{S}_{\Xi_{c c}^{+}} / \mathcal{S}_{\Lambda_{c}}$ & $\mathcal{S}_{\Xi_{c c}^{++}}$ & $\mathcal{S}_{\Xi_{c c}^{++}} / \mathcal{S}_{\Lambda_{c}}$ \\
\hline \hline $210 \mathrm{MeV} / c^{2}$ Mass Range & $15 \mathrm{fb}$ & $13 \times 10^{-4}$ & $24 \mathrm{fb}$ & $22 \times 10^{-4}$ \\
$210 \mathrm{MeV} / c^{2}$ Mass Range, $p^{*}$ Req. & $4.4 \mathrm{fb}$ & $5.6 \times 10^{-4}$ & $5.5 \mathrm{fb}$ & $6.9 \times 10^{-4}$ \\
Within 5 MeV/c $c^{2}$ of SELEX & $7.5 \mathrm{fb}$ & $6.9 \times 10^{-4}$ & $11 \mathrm{fb}$ & $10 \times 10^{-4}$ \\
Within 5 MeV/c $c^{2}$ of SELEX, $p^{*}$ Req. & $2.2 \mathrm{fb}$ & $2.7 \times 10^{-4}$ & $3.2 \mathrm{fb}$ & $4.0 \times 10^{-4}$ \\
\hline
\end{tabular}

members have done a similar analysis searching for double-charm baryons decaying to $\Xi_{c}^{0} \pi^{+}$and $\Xi_{c}^{0} \pi^{+} \pi^{+}[4]$. They also find no evidence for double-charm baryons. 


\section{Chapter 4}

\section{Charm-Strange Baryons}

All theoretical ground states of charm baryons containing one charm quark and two lighter quarks are believed to have been experimentally observed $[7,8]$. Theoretical mass spectra for ground-state charm baryons agree fairly well with experimental observation and are used to assign quantum numbers to these baryons. Each of these ground states may have orbital and/or radial excitations, which constitute new baryon states. Several of these excited charm baryons have been experimentally observed [7]. Further experimental study of these excited charm baryons provides valuable information to the theoretical understanding of quark dynamics.

The charm-strange baryons are denoted as $\Xi_{c}$. Excited charm-strange baryons that have been previously observed are $\Xi_{c}(2790)^{+, 0}, \Xi_{c}(2815)^{+, 0}, \Xi_{c}(2980)^{+}$, and $\Xi_{c}(3077)^{+, 0}[7,6]$. Each of these excited charm-strange baryon states are illustrated in Figure 1.2. Prior to the recent observations of $\Xi_{c}(2980)^{+}$and $\Xi_{c}(3077)^{+, 0}$ in 2006, all known excited charm-strange baryons had been observed only in decays to lower-mass $\Xi_{c}$ baryons plus a pion or gamma. The two states $\Xi_{c}(2980)^{+}$and $\Xi_{c}(3077)^{+, 0}$ were observed in final states in which the charm and strange quarks are contained in separate hadrons and the isospin of the daughter baryon is different from that of the $I=1 / 2$ isospin of the excited charm-strange baryon. These types of decays may have implications as to the internal quark dynamics of these two states. Several excited charm-strange baryons with $J^{P}=\left\{\frac{1}{2}^{+}, \frac{1}{2}^{-}, \frac{3}{2}^{+}, \frac{3}{2}^{-}\right\}$ have been predicted to have masses ranging from about $2800 \mathrm{MeV} / c^{2}$ to $3150 \mathrm{MeV} / c^{2}[14,13]$. Spin and parity quantum numbers for excited charm-strange baryons have never been measured, however, and comparisons with theoretical mass spectra, decay modes, and decay rates are not conclusive enough to suggest quantum numbers for each observed excited charm-strange baryon.

In this chapter, I present my study of the excited charm-strange baryons that decay to final states in which the charm and strange quarks are contained in separate hadrons. I search for decays to the three-body final states $\Lambda_{c}^{+} K^{-} \pi^{+}$and $\Lambda_{c}^{+} K_{s} \pi^{-}$, the two-body final states $\Lambda_{c}^{+} K_{s}$ and $\Lambda_{c}^{+} K^{-}$, and the four-body final states $\Lambda_{c}^{+} K_{s} \pi^{-} \pi^{+}$and $\Lambda_{c}^{+} K^{-} \pi^{-} \pi^{+}$. The intermediate-resonant states $\Sigma_{c}(2455)^{++} K^{-}, \Sigma_{c}(2455)^{0} K_{s}, \Sigma_{c}(2520)^{++} K^{-}$, and $\Sigma_{c}(2520)^{0} K_{s}$ are also searched for in the 
three-body final states. Measurements of the mass, width, yield, and cross-section times branching fractions are made in cases where there is evidence for excited charm-strange baryons. Also, where applicable, the intermediate-resonant decay fractions are measured.

\subsection{Data and Monte-Carlo Samples}

I use a data sample corresponding to an integrated luminosity of $380 \mathrm{fb}^{-1}$ for my analysis of excited charm-strange baryons. I employ five decay modes of the $\Lambda_{c}^{+}$for this study: $p K^{-} \pi^{+}, p K_{s}, p K_{s} \pi^{-} \pi^{+}$, $\Lambda^{0} \pi^{+}$, and $\Lambda^{0} \pi^{+} \pi^{-} \pi^{+}$. The $\Lambda^{0}$ and $K_{s}$ candidates are reconstructed from their decays to $p \pi^{-}$and $\pi^{+} \pi^{-}$, respectively. The $\Lambda^{0}$ and $K_{s}$ are relatively long lived hadrons, and they typically travel centimeters before they decay in the $B A B A R$ Detector. After an initial vertex constrained fit of tracks to form $\Lambda^{0}$ and $K_{s}$ candidate, $\Lambda^{0}$ and $K_{s}$ candidates are refit with mass constraints of $1115.683 \mathrm{MeV} / c^{2}$ and $497.648 \mathrm{MeV} / c^{2}$, respectively, which are their world-average values [7]. All reconstructed $\Lambda_{c}^{+}$candidates are also refit with a mass constraint to the $\Lambda_{c}^{+}$world average mass of $2286.46 \mathrm{MeV} / \mathrm{c}^{2}[7]$.

Ten different combinations of kaons and pions are combined in fits with the mass-constrained $\Lambda_{c}^{+}$candidates. The "right-sign" combinations, which correspond to possible excited charm-strange baryon decay modes, are $\Lambda_{c}^{+} K^{-} \pi^{+} \pi^{-}, \Lambda_{c}^{+} K^{-} \pi^{+}, \Lambda_{c}^{+} K^{-}, \Lambda_{c}^{+} K_{s} \pi^{+} \pi^{-}, \Lambda_{c}^{+} K_{s} \pi^{-}$, and $\Lambda_{c}^{+} K_{s}$. The "wrong-sign" combinations are $\Lambda_{c}^{+} K^{+}, \Lambda_{c}^{+} K^{+} \pi^{-}$, and $\Lambda_{c}^{+} K^{+} \pi^{-} \pi^{-}$. The wrong-sign candidates do not constitute valid baryons and they are used to represent combinatorial backgrounds (random combinations of tracks).

Signal-MC samples of excited charm-strange baryons are created using JETSET74 to simulate $e^{+} e^{-} \rightarrow \gamma \rightarrow c \bar{c}$ production and quark hadronization. All simulated excited charm-strange baryons are forced to decay into only the decay channels that are studied in this analysis. The $\Lambda_{c}^{+}$baryons in the signal-MC samples decay $53.34 \%$ into $p K^{-} \pi^{+}, 8.46 \%$ into $p K_{s}, 9.56 \%$ into $p K_{s} \pi^{+} \pi^{-}, 6.13 \%$ into $\Lambda^{0} \pi^{+}$, and $22.50 \%$ into $\Lambda^{0} \pi^{+} \pi^{-} \pi^{+}$. The charm-strange baryon states are simulated in MC with masses and natural widths as listed in Table 4.1. For simulated excited charm-strange baryon decays with two daughter particles, EvtGen uses a relativistic Breit-Wigner with a mass dependent width proportional to

$$
\frac{1}{\left(M^{2}-M_{0}^{2}\right)^{2}+\left(M_{0} \frac{p}{p^{\prime}} \Gamma\right)^{2}},
$$

where $M$ is the mass, $M_{0}$ is the pole mass, $\Gamma$ is the constant width, $p$ is the daughter momentum in the parent rest frame, and $p^{\prime}$ is the same but calculated using the pole mass of the parent. For simulated excited charm-strange baryon decays with more than two daughter hadrons, this functional form is ill-defined, and the MC generator EvtGen uses a more generic non-relativistic Breit-Wigner mass distribution proportional to

$$
\frac{1}{\left(M-M_{0}\right)^{2}+\Gamma^{2} / 4} \text {. }
$$


Table 4.1: Simulated charm-strange baryon masses and natural widths.

\begin{tabular}{lcccc}
\hline & $\Xi_{c}(2970)^{+, 0}$ & $\Xi_{c}(3055)^{+, 0}$ & $\Xi_{c}(3077)^{+, 0}$ & $\Xi_{c}(3123)^{+, 0}$ \\
\hline \hline Mass $\left(\mathrm{MeV} / c^{2}\right)$ & 2967.1 & 3054.3 & 3076.4 & 3123.0 \\
Width $(\mathrm{MeV})$ & 23.6 & 19.4 & 6.2 & 3.9 \\
\hline \hline
\end{tabular}

Table 4.2: Signal-MC samples for charm-strange baryons. Number of generated signal-MC candidates, number of truth-matched signal-MC candidates passing the reconstruction criteria, and their ratio.

\begin{tabular}{lccc}
\hline Signal Mode & Number Generated & Number Found & Efficiency (\%) \\
\hline \hline$\Xi_{c}(2970)^{0} \rightarrow \Lambda_{c}^{+} K^{-}$ & 383,147 & 85,968 & 22.4 \\
$\Xi_{c}(3077)^{0} \rightarrow \Lambda_{c}^{+} K^{-}$ & 383,035 & 87,128 & 22.7 \\
$\Xi_{c}(2970)^{+} \rightarrow \Lambda_{c}^{+} K^{-} \pi^{+}$ & 383,166 & 72,071 & 18.8 \\
$\Xi_{c}(3077)^{+} \rightarrow \Lambda_{c}^{+} K^{-} \pi^{+}$ & 383,042 & 71,289 & 18.6 \\
$\Xi_{c}(3077)^{0} \rightarrow \Lambda_{c}^{+} K^{-} \pi^{+} \pi^{-}$ & 383,024 & 74,728 & 19.5 \\
$\Xi_{c}(2970)^{+} \rightarrow \Lambda_{c}^{+} K_{s}$ & 383,141 & 51,292 & 13.4 \\
$\Xi_{c}(3077)^{+} \rightarrow \Lambda_{c}^{+} K_{s}$ & 383,032 & 52,686 & 13.8 \\
$\Xi_{c}(2970)^{0} \rightarrow \Lambda_{c}^{+} K_{s} \pi^{-}$ & 383,157 & 37,921 & 9.9 \\
$\Xi_{c}(3077)^{0} \rightarrow \Lambda_{c}^{+} K_{s} \pi^{-}$ & 383,035 & 39,457 & 10.3 \\
$\Xi_{c}(3077)^{+} \rightarrow \Lambda_{c}^{+} K_{s} \pi^{+} \pi^{-}$ & 383,035 & 36,666 & 9.6 \\
\hline \hline
\end{tabular}

A feature of the signal-MC generation is that the generated mass of the $\Xi_{c}(2970)^{+} \rightarrow \Lambda_{c}^{+} K^{-} \pi^{+}$ is restricted to be within $\pm 47.39 \mathrm{MeV} / c^{2}$ of $2967.1 \mathrm{MeV} / c^{2}$ due to the kinematic limit at $M\left(\Lambda_{c}^{+}\right)+$ $M\left(K^{-}\right)+M\left(\pi^{+}\right)=2919.71 \mathrm{MeV} / c^{2}$. Naturally, this kinematic limit is only on the low mass side, but the limits of the signal-MC generation are symmetric around the pole mass. Similarly, the simulated decays $\Xi_{c}(2970)^{0} \rightarrow \Lambda_{c}^{+} K_{s} \pi^{-}, \Xi_{c}(3077)^{0} \rightarrow \Lambda_{c}^{+} K^{-} \pi^{+} \pi^{-}$, and $\Xi_{c}(3077)^{+} \rightarrow \Lambda_{c}^{+} K_{s} \pi^{-} \pi^{+}$ all have invariant-mass distributions with a kinematic lower limit and a symmetric upper limit. The $\Xi_{c}(2970)$ state is not kinematically allowed to decay to $\Lambda_{c}^{+} K_{s} \pi^{-} \pi^{+}$or $\Lambda_{c}^{+} K^{-} \pi^{+} \pi^{-}$and is not simulated as such. Other simulated excited charm-strange baryon decays are relatively far from the kinematic limits. All hadrons are simulated to decay according to $N$-body phase space. The signalMC samples are analyzed in the same manner as the data. The numbers of generated signal-MC candidates are listed in Table 4.2. Also listed are the numbers of and efficiencies for true signalMC candidates being reconstructed and passing the reconstruction criteria. A truth-matching error causes the number of $\Lambda_{c}^{+} K^{-} \pi^{+} \pi^{-}$to be over estimated by a few percent, but this error does not affect the results of this analysis in any way. 


\subsection{Candidate Selection}

When reconstructing signal candidates, tracks are required to pass some basic requirements before they are considered. This reduces the number of track combinations considered to a manageable level without being so stringent as to significantly reduce signal reconstruction efficiencies. To be considered as a proton or kaon, a track must pass the lowest level of the PID likelihood criteria, VeryLoose. Any track is considered as a pion because most tracks are from charged pions. The daughter tracks of $\Lambda^{0}$ and $K_{s}$ candidates are not required to pass PID likelihood criteria because these relatively long lived hadrons can be effectively identified by their flight length. $\Lambda^{0}$ and $K_{s}$ hadrons typically have flight lengths on the order of $1 \mathrm{~cm}$. The flight length of a candidate is defined as the distance, in the direction of the candidates momentum, between the $e^{+} e^{-}$interaction region and the candidates decay vertex. Candidates originating from outside the $e^{+} e^{-}$interaction region (such as candidates from cosmic rays) often have negative flight lengths. A $\Lambda^{0}$ or $K_{s}$ candidate is required to have a measured flight length greater than zero to be further considered after if has been reconstructed. The $\Lambda^{0}$ and $K_{s}$ vertex fit $\chi^{2}$ probabilities are required to be greater than $0.1 \%$. A $\Lambda^{0}$ candidate is rejected if its invariant mass is not within a wide range $\left(1106 \mathrm{MeV} / \mathrm{c}^{2}\right.$ and $1125 \mathrm{MeV} / c^{2}$ ) around the world average $\Lambda^{0}$ mass. A $K_{s}$ candidate is rejected if its invariant mass is not within a wide range $\left(486 \mathrm{MeV} / c^{2}\right.$ and $\left.510 \mathrm{MeV} / c^{2}\right)$ around the world average $K_{s}$ mass. The $\Lambda^{0}$ and $K_{s}$ candidates, along with proton candidates passing the VeryLoose PID criterion, kaon candidates passing the VeryLoose PID criterion, and pion candidates (any track), are used to

reconstruct the $\Lambda_{c}^{+}$candidates. To be further considered as a $\Lambda_{c}^{+}$, candidates are required to have a reconstructed mass within a wide range (between $2235 \mathrm{MeV} / c^{2}$ and $2335 \mathrm{MeV} / c^{2}$ ) around the world average $\Lambda_{c}^{+}$mass and a vertex-fit $\chi^{2}$ probability of greater that 0.001 . The excited charm-strange baryon candidates are reconstructed from $\Lambda_{c}^{+}$candidates and additional kaon and pion tracks that pass the same criteria as the other kaons and pions just described. The only other requirement of a reconstructed excited charm-strange baryon candidate is that its $p^{*}$ be greater than $2.0 \mathrm{GeV} / c$. This requirement greatly reduces the amount of combinatorial background in the sample, without significantly reducing the signal reconstruction efficiency.

After the reconstruction of signal candidates, twelve discrimination variables are further studied to find a set of selection criteria that maximizes the significance of potential signals in the data as described below. Signal-MC events are used to estimate the number of candidates that satisfy different sets of selection criteria. Candidates, from the data, in the invariant-mass side bands to the signal region are used to estimate the number of background candidates that satisfy different sets of selection criteria. Side-band regions are chosen to contain large numbers of candidates are well as to be near the signal region. The different side-band regions are listed in Table 4.3. The sideband regions are not used in the final fits to the data. The number of signal-MC candidates in the signal regions $\left(S_{i}\right)$ and background candidates in the side-band regions $\left(B_{i}\right)$ passing a set of selection criteria are counted separately for each of the five reconstructed $\Lambda_{c}^{+}$decay modes $(i=1,2,3,4,5)$. I 
Table 4.3: Invariant-mass side-band regions used to estimate the amount of background in the various excited charm-strange baryon decay modes.

\begin{tabular}{cc}
\hline Invariant Masses & Side-Band Ranges \\
\hline \hline$M\left(\Lambda_{c}^{+} K^{-}\right)$and $M\left(\Lambda_{c}^{+} K_{s}\right)$ & $2.75 \mathrm{GeV} / c^{2}-2.85 \mathrm{GeV} / c^{2}$ and $3.15 \mathrm{GeV} / c^{2}-3.25 \mathrm{GeV} / c^{2}$ \\
$M\left(\Lambda_{c}^{+} K^{-} \pi^{+}\right)$and $M\left(\Lambda_{c}^{+} K_{s} \pi^{-}\right)$ & $3.15 \mathrm{GeV} / c^{2}-3.25 \mathrm{GeV} / c^{2}$ \\
$M\left(\Lambda_{c}^{+} K^{-} \pi^{+} \pi^{-}\right)$and $M\left(\Lambda_{c}^{+} K_{s} \pi^{-} \pi^{+}\right)$ & $3.15 \mathrm{GeV} / c^{2}-3.30 \mathrm{GeV} / c^{2}$ \\
\hline \hline
\end{tabular}

assume in this analysis that background events dominate the data sample in the signal region so that the uncertainty on the number of signal candidates is approximately $\sqrt{B}$. A combined estimated significance is calculated as the weighted average of the number of signal candidates $\left(S_{i} \pm \sqrt{B_{i}}\right)$ divided by the error on the weighted average:

$$
\left(\frac{\sum_{i} S_{i} / B_{i}}{\sum_{i} 1 / B_{i}}\right) /\left(\sum_{i} 1 / B_{i}\right)^{-1 / 2}
$$

Selection criteria producing the highest significance for each of the six MC signal decay modes are listed in Table 4.4. The overall scale of the estimated significance is arbitrary and does not affect the study; only the relative differences of estimated significance between different sets of selection criteria affect the study.

The criteria levels for each of the twelve discrimination variables are simultaneously and independently varied to be both more and less stringent about their starting criteria levels. The set of criteria levels that maximizes the estimated significance is then used as the initial set for further variations of selection criteria. This iterative process is repeated until the selection criteria provide the largest estimated significance of all the sets of criteria. Table 4.4 lists for each of the six signal decay modes used in this analysis the set of selection criteria that maximizes the estimated significance.

The $P\left(\chi^{2}\right)$ variable is the combined $\chi^{2}$ probability for all vertex and mass-constrained fits of each candidate and its decay products. The minimum $P\left(\chi^{2}\right)$ values that are tested are $0.01,0.05$, $0.1,0.5,1.0,1.5,2.0,2.5$, and 3.0 percent. The row labeled "Track Quality" in Table 4.4 refers to the minimum BABAR standard tracking quality required for all candidate tracks not associated with a $K_{s}$ or $\Lambda^{0}$. "Acceptance Angle" is 1 if all candidate tracks not associated with a $K_{s}$ or $\Lambda^{0}$ have a polar angle $\theta$ (with respect to the z-axis) between $0.410 \mathrm{rad}$ and $2.54 \mathrm{rad}$; it is zero otherwise. The variable $p^{*}$ refers to the momentum of the signal candidate in the $e^{+} e^{-}$center-of-mass frame. The minimum allowed value is varied in $0.1 \mathrm{GeV} / c$ increments. The $M\left(\Lambda_{c}^{+}\right)$resolution is about $6 \mathrm{MeV} / c^{2}$ for the reconstruction of $\Lambda_{c}^{+}$decays to $p K^{-} \pi^{+}, p K_{s}$, and $\Lambda^{0} \pi^{+}$. It is about $4 \mathrm{MeV} / c^{2}$ for the reconstruction of $\Lambda_{c}^{+}$decays to $p K_{s} \pi^{+} \pi^{-}$and $\Lambda^{0} \pi^{+} \pi^{-} \pi^{+}$. The allowed $\Lambda_{c}^{+}$mass ranges 
are increased and decreased in $0.5 \sigma$ increments of width around the $\Lambda_{c}^{+}$mass of $2286.46 \mathrm{MeV} / \mathrm{c}^{2}$. Accepted ranges for $M\left(K_{s}\right)$ and $M(\Lambda)$ are varied by $1.0 \mathrm{MeV} / c^{2}$ increments around the $K_{s}$ mass of $497.648 \mathrm{MeV} / c^{2}$ and the $\Lambda^{0}$ mass of $1115.683 \mathrm{MeV} / c^{2} . F\left(K_{s}\right)$ and $F(\Lambda)$ are the minimum allowed values of flight length significances for a $K_{s}$ or a $\Lambda^{0}$. The $p, K$, and $\pi$ PID likelihood selectors apply to all tracks.

Given the limited variation in criteria sets that maximize the estimated significance for each of the six signal decay modes, one set of criteria is used for all signal modes. These criteria are listed in the last column of Table 4.4. This choice of a single set of selection criteria does not significantly reduce the estimated significances for any of the signal decay modes. The largest reduction of estimated significance is for $\Lambda_{c}^{+} K_{s} \pi^{-} \pi^{+}$and is about $10 \%$. The third and fourth to last rows of Table 4.4 list the best estimated significance found for each decay mode, resulting from the selection criteria given in the corresponding column, and the estimated significance for each decay mode using the selection criteria listed in the last column.

The number of candidates selected per event is measured for each $\Lambda_{c}^{+}$decay mode in the signalMC sample. Low candidate multiplicities are found for all excited charm-strange baryon decays. Candidate multiplicities for $\Xi_{c}(2970)^{+, 0}$ and $\Xi_{c}(3077)^{+, 0}$ are listed in Table 4.5. Comparing the invariant mass distributions for false signal-MC candidates from events with one candidate and for those from events with multiple candidates reveals no differences in shape. This indicates that multiple candidates in one event do not create additional background contributions or distort the signal shape.

\subsection{Three-Body Decays}

The study of excited charm-strange baryons decaying to $\Lambda_{c}^{+} K^{-} \pi^{+}$and $\Lambda_{c}^{+} K_{s} \pi^{-}$is described in this section. These are three-body decays, and their daughter hadrons may be born with a range of momenta known as phase space. For a three-body decay $Z \rightarrow A B C$, the phase space can be parametrized by five independent variables: $M_{a b}^{2} \equiv M(A B)^{2}$ and $M_{b c}^{2} \equiv M(B C)^{2}$, which are invariant-mass squared of the daughter combinations $A B$ and $B C$, and the Euler angles $\alpha, \beta$, and

$\gamma$ between the parent and daughter particles. The partial decay rate $(d \Gamma)$ for a three-body decay into a point of this phase space $\left(d\left(M_{a b}^{2}\right) d\left(M_{b c}^{2}\right) d \alpha d(\cos \beta) d \gamma\right)$ is directly proportional to the squared magnitude of the Lorentz invariant decay amplitude $(\mathcal{M})$;

$$
d \Gamma \propto|\mathcal{M}|^{2} d\left(M_{a b}^{2}\right) d\left(M_{b c}^{2}\right) d \alpha d(\cos \beta) d \gamma .
$$

If a decay amplitude is averaged over its spin states, the average amplitude has no dependence on the relative angles between the parent particle and the daughters;

$$
d \Gamma \propto \overline{|\mathcal{M}|^{2}} d\left(M_{a b}^{2}\right) d\left(M_{b c}^{2}\right) .
$$


Table 4.4: Selection criteria producing the highest significance for each of the six MC signal decay modes. The entries in rows labeled $\Delta M$ are allowed mass differences from a central mass value, and entries in rows labeled $F$ are minimum flight length significances. The last column indicates the selection criteria that are chosen to be applied to all signal candidate types. The third and fourth to last rows list the best estimated significance found for each decay mode resulting from the selection criteria given in the corresponding column, and the estimated significances for each decay mode using the selection criteria listed in the last column. The estimated significances are scaled down by a factor of 100; the scale of the estimated significance is arbitrary because only their relative values affect the analysis. The last two rows give the efficiencies for the selection criteria listed in each column to accept signal candidates and to accept background candidates.

\begin{tabular}{lccccccc}
\hline Variable & $\Lambda_{c} K$ & $\Lambda_{c} K \pi$ & $\Lambda_{c} K \pi \pi$ & $\Lambda_{c} K_{s}$ & $\Lambda_{c} K_{s} \pi$ & $\Lambda_{c} K_{s} \pi \pi$ & All \\
\hline \hline$P\left(\chi^{2}\right)(\%)$ & 0.05 & 0.5 & 0.5 & 1.0 & 2.0 & 0.1 & 1.0 \\
Track Quality & $\mathrm{L}$ & $\mathrm{VL}$ & $\mathrm{VL}$ & $\mathrm{VL}$ & $\mathrm{VT}$ & $\mathrm{VL}$ & $\mathrm{VL}$ \\
Acceptance Angle & 1 & 0 & 0 & 1 & 0 & 0 & 0 \\
$p *(\mathrm{GeV} / c)$ & 2.8 & 2.9 & 2.9 & 2.9 & 3.0 & 3.1 & 2.9 \\
$\Delta M\left(\Lambda_{c}^{+}\right)(\#$ of $\sigma)$ & 1.75 & 1.75 & 2.00 & 1.50 & 1.75 & 2.25 & 1.75 \\
$\Delta M\left(K_{s}\right)\left(\mathrm{MeV} / c^{2}\right)$ & 9.0 & 9.5 & 11.5 & 9.5 & 10.5 & 9.5 & 10.0 \\
$\Delta M(\Lambda)\left(\mathrm{MeV} / c^{2}\right)$ & 5.5 & 8.0 & 5.5 & 6.5 & 6.5 & 8.5 & 6.5 \\
$F\left(K_{s}\right)(\#$ of $\sigma)$ & 1.5 & 2.5 & 2.0 & 4.5 & 4.0 & 4.5 & 3.0 \\
$F(\Lambda)(\#$ of $\sigma)$ & 3.0 & 3.0 & 2.5 & 2.5 & 2.5 & 2.5 & 2.5 \\
$p$ PID & $\mathrm{VT}$ & $\mathrm{VT}$ & $\mathrm{T}$ & $\mathrm{VT}$ & $\mathrm{VT}$ & $\mathrm{T}$ & $\mathrm{VT}$ \\
$K$ PID & $\mathrm{T}$ & $\mathrm{T}$ & $\mathrm{T}$ & $\mathrm{VT}$ & $\mathrm{T}$ & $\mathrm{L}$ & $\mathrm{T}$ \\
$\pi$ PID & $\mathrm{L}$ & $\mathrm{T}$ & $\mathrm{L}$ & $\mathrm{L}$ & $\mathrm{T}$ & $\mathrm{VL}$ & $\mathrm{L}$ \\
\hline Best Est. Sig. & 3.16 & 2.46 & 2.89 & 3.23 & 2.33 & 3.27 & - \\
Final Est. Sig. & 3.12 & 2.46 & 2.85 & 3.19 & 2.28 & 2.93 & - \\
\hline Signal Cut Eff. $(\%)$ & 38.9 & 33.8 & 33.5 & 38.8 & 32.9 & 22.4 & - \\
Bkgd. Cut Eff. $(\%)$ & 1.71 & 0.87 & 0.66 & 1.18 & 0.38 & 0.22 & - \\
\hline \hline
\end{tabular}

Table 4.5: Candidates per event for $\Xi_{c}(2970)^{+, 0}$ and $\Xi_{c}(3077)^{+, 0}$ signal MC.

\begin{tabular}{lcccc}
\hline Decay & $\Xi_{c}(2970)^{+}$ & $\Xi_{c}(3077)^{+}$ & $\Xi_{c}(2970)^{0}$ & $\Xi_{c}(3077)^{0}$ \\
\hline \hline Two-Body & 1.10 & 1.09 & 1.19 & 1.19 \\
Three-Body & 1.21 & 1.17 & 1.24 & 1.21 \\
Four-Body & - & 1.16 & - & 1.18 \\
\hline
\end{tabular}


The average three-body decay amplitude may be a constant throughout the kinematically allowed phase space of the two remaining variables. A nonconstant partial decay rate in this two dimensional phase space indicates a nonconstant decay amplitude and that some decay dynamics are preferential. This may be due to a intermediate-resonant state that a three-body decay proceeds through. In such a case, a three-body decay is in essence a chain of two two-body decays.

The amplitude of a three-body decay can be readily studied using a Dalitz plot. A Dalitz plot is a two-dimensional data distribution for the variables $M(A B)^{2}$ and $M(B C)^{2}$ of a three-body decay. If the average decay amplitude is constant, the kinematically allowed region of the Dalitz plot will be uniformly populated. A varying amplitude can result in a Dalitz plot with a higher density band of data along a constant value of $M(A B)^{2}, M(B C)^{2}$, or $M(C A)^{2}$. This would be an indication for an intermediate-resonance that then decays into $A B, B C$, or $C A$, respectively.

For the three-body decays $\Lambda_{c}^{+} K^{-} \pi^{+}$and $\Lambda_{c}^{+} K_{s} \pi^{-}$, there are only four kinematically allowed intermediate-resonance: $\Sigma_{c}(2455)^{++} \rightarrow \Lambda_{c}^{+} \pi^{+}, \Sigma_{c}(2520)^{++} \rightarrow \Lambda_{c}^{+} \pi^{+}, \Sigma_{c}(2455)^{0} \rightarrow \Lambda_{c}^{+} \pi^{-}$, and $\Sigma_{c}(2520)^{0} \rightarrow \Lambda_{c}^{+} \pi^{-}$. Intermediate-resonances of excited kaons decaying to $K^{-} \pi^{+}$or $K_{s} \pi^{-}$are not kinematically allowed. Figures 4.1 through 4.4 show Dalitz plots of $\Lambda_{c}^{+} K^{-} \pi^{+}$and $\Lambda_{c}^{+} K_{s} \pi^{-}$ candidates in data for all five $\Lambda_{c}^{+}$decay modes combined; the candidates in these figures are selected to have invariant mass near the signals for $\Xi_{c}(2970)$ states (Figures 4.1 and 4.3) and near the signals for $\Xi_{c}(3077)$ states (Figures 4.2 and 4.4). In Figures 4.1 through $4.4, \Sigma_{c}(2455)^{0,++}$ and $\Sigma_{c}(2520)^{0,++}$ intermediate resonances can be seen as vertical bands in the data distributions at $M\left(\Lambda_{c}^{+} \pi^{ \pm}\right)^{2}=6.03 \mathrm{GeV}^{2} / \mathrm{c}^{4}$ and $6.35 \mathrm{GeV}^{2} / \mathrm{c}^{4}$, respectively.

The Dalitz plot distributions in Figures 4.1 through 4.4 indicate that it is possible that threebody decays of excited charm-strange baryons are proceeding through intermediate-resonant states. It is also possible that the high density bands in these Dalitz plots are entirely due to combinatorial background candidates with real $\Sigma_{c}(2455)^{0,++}$ and $\Sigma_{c}(2520)^{0,++}$ baryons. In order to further investigate this, I study the three-body decay to $\Lambda_{c}^{+} K^{-} \pi^{+}$and $\Lambda_{c}^{+} K_{s} \pi^{-}$using twodimensional data distributions. These two dimensions are $M\left(\Lambda_{c}^{+} K_{s} \pi^{-}\right)$versus $M\left(\Lambda_{c}^{+} \pi^{-}\right)$, and $M\left(\Lambda_{c}^{+} K^{-} \pi^{+}\right)$versus $M\left(\Lambda_{c}^{+} \pi^{+}\right)$. Using these two dimensions, I am able to distinguish between background, excited charm-strange baryon signal, and intermediate-resonant signal, distributions: background does not peak in $M\left(\Lambda_{c}^{+} K^{-} \pi^{+}\right)$or $M\left(\Lambda_{c}^{+} K_{s} \pi^{-}\right)$, excited charm-strange baryon signal peaks in $M\left(\Lambda_{c}^{+} K^{-} \pi^{+}\right)$or $M\left(\Lambda_{c}^{+} K_{s} \pi^{-}\right)$, and intermediate-resonant signal peaks in $M\left(\Lambda_{c}^{+} K^{-} \pi^{+}\right)$ and $M\left(\Lambda_{c}^{+} \pi^{+}\right)$, or $M\left(\Lambda_{c}^{+} K_{s} \pi^{-}\right)$and $M\left(\Lambda_{c}^{+} \pi^{-}\right)$.

In this section, I use the following notation:

$$
\begin{gathered}
M_{\Xi_{c}} \equiv M\left(\Lambda_{c}^{+} K_{s} \pi^{-}\right) \text {or } M\left(\Lambda_{c}^{+} K^{-} \pi^{+}\right), \\
M_{\Sigma_{c}} \equiv M\left(\Lambda_{c}^{+} \pi^{-}\right) \text {or } M\left(\Lambda_{c}^{+} \pi^{+}\right) .
\end{gathered}
$$

This study utilizes unbinned, extended maximum-likelihood fits. The physically allowed kinematic 


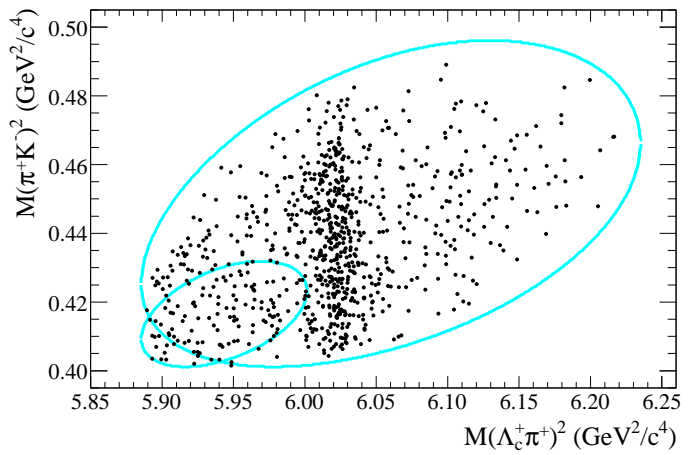

Figure 4.1: Dalitz plot of $M\left(\pi^{+} K^{-}\right)^{2}$ versus $M\left(\Lambda_{c}^{+} \pi^{+}\right)^{2}$ for $\Xi_{c}(2970)^{+}$candidates in data, from all five $\Lambda_{c}^{+}$decay modes combined. The curves represent the kinematic boundaries of the Dalitz plot for the upper and lower $M_{\Xi_{c}}$ limits used for the $\Xi_{c}(2970)^{+}$signal.

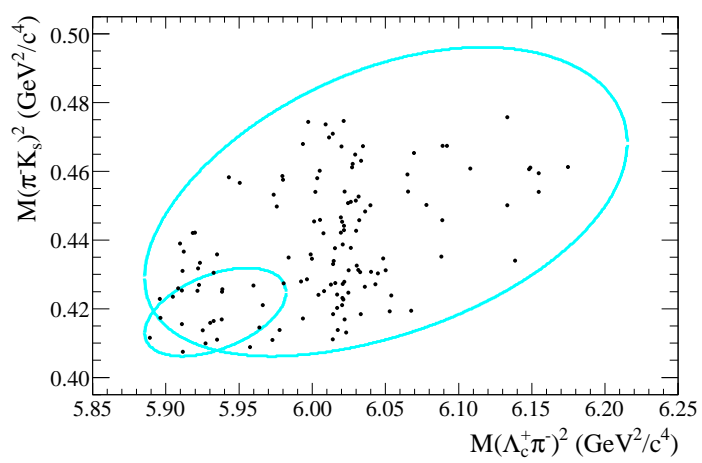

Figure 4.3: Dalitz plot of $M\left(\pi^{-} K_{s}\right)^{2}$ versus $M\left(\Lambda_{c}^{+} \pi^{-}\right)^{2}$ for $\Xi_{c}(2970)^{0}$ candidates in data, from all five $\Lambda_{c}^{+}$decay modes combined. The curves represent the kinematic boundaries of the Dalitz plot for the upper and lower $M_{\Xi_{c}}$ limits used for the $\Xi_{c}(2970)^{0}$ signal.

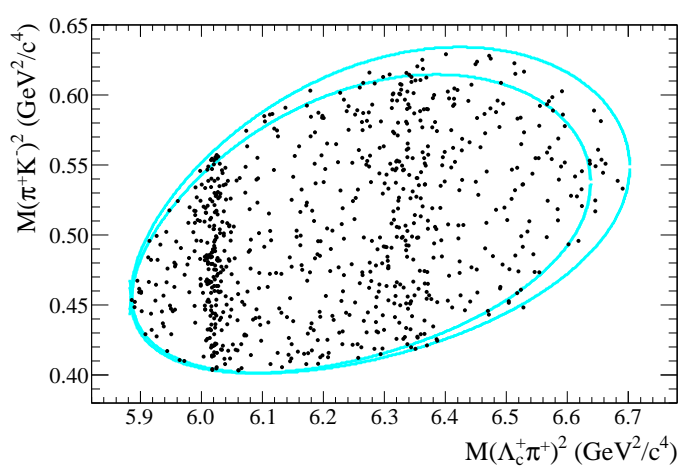

Figure 4.2: Dalitz plot of $M\left(\pi^{+} K^{-}\right)^{2}$ versus $M\left(\Lambda_{c}^{+} \pi^{+}\right)^{2}$ for $\Xi_{c}(3077)^{+}$candidates in data, from all five $\Lambda_{c}^{+}$decay modes combined. The curves represent the kinematic boundaries of the Dalitz plot for the upper and lower $M_{\Xi_{c}}$ limits used for the $\Xi_{c}(3077)^{+}$signal.

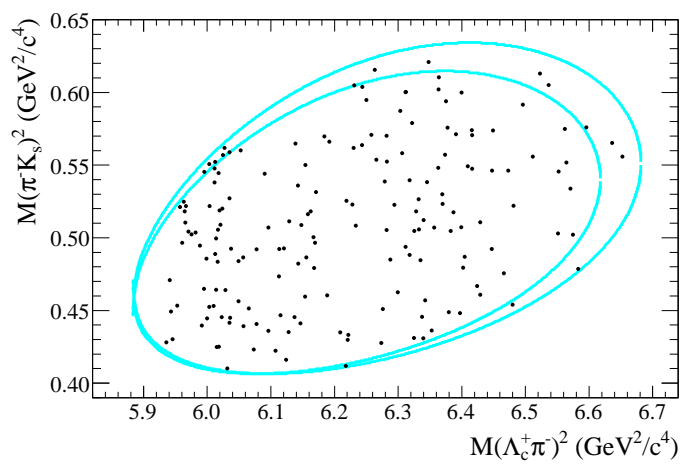

Figure 4.4: Dalitz plot of $M\left(\pi^{-} K_{s}\right)^{2}$ versus $M\left(\Lambda_{c}^{+} \pi^{-}\right)^{2}$ for $\Xi_{c}(3077)^{0}$ candidates in data, from all five $\Lambda_{c}^{+}$decay modes combined. The curves represent the kinematic boundaries of the Dalitz plot for the upper and lower $M_{\Xi_{c}}$ limits used for the $\Xi_{c}(3077)^{0}$ signal. 
Table 4.6: $M\left(\Lambda_{c}^{+} K_{s} \pi^{-}\right)$and $M\left(\Lambda_{c}^{+} K^{-} \pi^{+}\right)$mass resolutions for three-body decay modes. $\sigma_{N}$ refers to the width of the narrower of the two Gaussians; $\sigma_{W}$ refers to the wider of the two. The fraction of each sample that is fit by the narrower Gaussian is also given.

\begin{tabular}{lccc}
\hline Signal & $\sigma_{N}\left(\mathrm{MeV} / c^{2}\right)$ & $\sigma_{W}\left(\mathrm{MeV} / c^{2}\right)$ & Frac. $(\%)$ \\
\hline \hline$\Xi_{c}(2970)^{+}$ & $0.97 \pm 0.02$ & $2.51 \pm 0.06$ & $70 \pm 2$ \\
$\Xi_{c}(3055)^{+}$ & $1.53 \pm 0.05$ & $3.45 \pm 0.10$ & $65 \pm 3$ \\
$\Xi_{c}(3077)^{+}$ & $1.71 \pm 0.02$ & $4.58 \pm 0.13$ & $72 \pm 2$ \\
$\Xi_{c}(3123)^{+}$ & $1.71 \pm 0.03$ & $3.91 \pm 0.07$ & $58 \pm 2$ \\
$\Xi_{c}(2970)^{0}$ & $0.93 \pm 0.02$ & $2.38 \pm 0.07$ & $69 \pm 2$ \\
$\Xi_{c}(3055)^{0}$ & $1.63 \pm 0.06$ & $2.85 \pm 0.15$ & $73 \pm 4$ \\
$\Xi_{c}(3077)^{0}$ & $1.69 \pm 0.03$ & $4.39 \pm 0.16$ & $74 \pm 2$ \\
$\Xi_{c}(3123)^{0}$ & $1.68 \pm 0.05$ & $3.77 \pm 0.09$ & $60 \pm 3$ \\
\hline
\end{tabular}

ranges of these invariant masses below $M_{\Xi_{c}}=3150 \mathrm{MeV} / c^{2}$ are fit. The data is divided into five subsamples based on the reconstructed $\Lambda_{c}^{+}$decay mode: $p K^{-} \pi^{+}, p K_{s}, p K_{s} \pi^{-} \pi^{+}, \Lambda^{0} \pi^{+}$, or $\Lambda^{0} \pi^{+} \pi^{-} \pi^{+}$. All five subsamples are fit simultaneously with shared PDF parameters for signal shape.

The use of MC samples to measure detector resolutions and signal reconstruction efficiencies is described in Section 4.3.1. The PDF components used to fit the data are described in Section 4.3.2. The likelihood function and the systematic errors are described in Sections 4.3.3 and 4.3.4. The $\Lambda_{c}^{+} K^{-} \pi^{+}$analysis results are described in Section 4.3.5 and the $\Lambda_{c}^{+} K_{s} \pi^{-}$analysis in Section 4.3.6.

\subsubsection{Mass Resolution and Signal Efficiency}

For the $M_{\Xi_{c}}$ and $M_{\Sigma_{c}}$ invariant-mass distributions, two Gaussian functions with a common mean are used to represent the mass resolution. The widths and relative fractions of the two Gaussian resolution functions are determined from signal-MC studies where the difference between the simulated mass and the reconstructed mass is measured for each candidate. The widths and relative fractions do not show any significant differences between the five $\Lambda_{c}^{+}$decay modes. The widths and relative fractions from the five $\Lambda_{c}^{+}$decay modes are averaged (wighted by their errors) and listed in Tables 4.6, 4.7, and 4.8. These averaged values and their errors are used to represent the mass resolution in the subsequent analysis. The statistical errors on the averaged resolutions are smaller than the $10 \%$ systematic error, which is discussed in Section 4.3.4. The averaged resolutions and fractions for $\Sigma_{c}(2455)^{0,++}$ that are listed in Tables 4.7 and 4.8 are subsequently averaged between the two tables for use in the PDF. The differences between the listed $\Sigma_{c}(2455)^{0,++}$ values are used for calculating the systematic uncertainty due to the use of the average.

Three-body decays through intermediate-resonant states not simulated in the signal-MC samples. This means that MC simulated signal candidates may not have the same distribution in a phase space as real signal, which may have intermediate-resonant states. This would cause a difficulty 
Table 4.7: $M\left(\Lambda_{c}^{+} \pi^{-}\right)$and $M\left(\Lambda_{c}^{+} \pi^{+}\right)$mass resolutions for $\Xi_{c}(3077)^{+, 0}$ reconstruction. $\sigma_{N}$ refers to the width of the narrower of the two Gaussians; $\sigma_{W}$ refers to the wider of the two. The fraction of each sample that is fit by the narrower Gaussian is also given.

\begin{tabular}{lccc}
\hline Signal & $\sigma_{N}\left(\mathrm{MeV} / c^{2}\right)$ & $\sigma_{W}\left(\mathrm{MeV} / c^{2}\right)$ & Frac. (\%) \\
\hline \hline$\Sigma_{c}(2455)^{0}$ & $0.55 \pm 0.03$ & $1.55 \pm 0.04$ & $40 \pm 3$ \\
\hline$\Sigma_{c}(2455)^{++}$ & $0.68 \pm 0.02$ & $2.12 \pm 0.05$ & $58 \pm 2$ \\
\hline$\Sigma_{c}(2520)^{0}$ & $0.87 \pm 0.03$ & $2.31 \pm 0.03$ & $40 \pm 2$ \\
\hline$\Sigma_{c}(2520)^{++}$ & $0.91 \pm 0.02$ & $2.29 \pm 0.03$ & $43 \pm 2$ \\
\hline \hline
\end{tabular}

Table 4.8: $M\left(\Lambda_{c}^{+} \pi^{-}\right)$and $M\left(\Lambda_{c}^{+} \pi^{+}\right)$mass resolutions for $\Xi_{c}(2970)^{0,+}$ reconstruction. $\sigma_{N}$ refers to the width of the narrower of the two Gaussians; $\sigma_{W}$ refers to the wider of the two. The fraction of each sample that is fit by the narrower Gaussian is also given.

\begin{tabular}{lccc}
\hline Signal & $\sigma_{N}\left(\mathrm{MeV} / c^{2}\right)$ & $\sigma_{W}\left(\mathrm{MeV} / c^{2}\right)$ & Frac. (\%) \\
\hline \hline$\Sigma_{c}(2455)^{0}$ & $0.49 \pm 0.02$ & $1.49 \pm 0.02$ & $45 \pm 2$ \\
\hline$\Sigma_{c}(2455)^{++}$ & $0.53 \pm 0.01$ & $1.50 \pm 0.02$ & $43 \pm 1$ \\
\hline \hline
\end{tabular}


Table 4.9: Reconstruction efficiencies, in percent, for $\Xi_{c}(2970)^{0} \rightarrow \Lambda_{c}^{+} K_{s} \pi^{-}$three-body decays. Efficiencies are given for each $\Lambda_{c}^{+}$decay mode and for two $M_{\Sigma_{c}}$ ranges. The efficiencies are listed with statistical errors only. The efficiency for each $\Lambda_{c}^{+}$decay mode averaged over the two $M_{\Sigma_{c}}$ ranges is listed with statistical (first) and systematic (second) errors.

\begin{tabular}{lccc}
\hline Decay Mode & $<2470 \mathrm{MeV} / c^{2}$ & $(2470-2550) \mathrm{MeV} / c^{2}$ & Average \\
\hline \hline$p K^{-} \pi^{+}$ & $1.51 \pm 0.02$ & $1.47 \pm 0.06$ & $1.49 \pm 0.02 \pm 0.08$ \\
\hline$p K_{s}$ & $0.43 \pm 0.01$ & $0.47 \pm 0.05$ & $0.47 \pm 0.01 \pm 0.03$ \\
\hline$p K_{s} \pi^{+} \pi^{-}$ & $0.16 \pm 0.01$ & $0.17 \pm 0.03$ & $0.18 \pm 0.01 \pm 0.02$ \\
\hline$\Lambda^{0} \pi^{+}$ & $0.74 \pm 0.03$ & $0.91 \pm 0.11$ & $0.84 \pm 0.03 \pm 0.06$ \\
\hline$\Lambda^{0} \pi^{+} \pi^{-} \pi^{+}$ & $0.32 \pm 0.01$ & $0.28 \pm 0.03$ & $0.35 \pm 0.01 \pm 0.04$ \\
\hline \hline
\end{tabular}

with estimating signal efficiency is the signal efficiecy varries with where in phase space an excited charm-strange baryon decays. I look for variations of efficiency in the Dalitz variables $M(A B)^{2}$, $M(B C)^{2}$, and $M(C A)^{2}$, where $A=\Lambda_{c}^{+}, B=\left\{K_{s}, K^{-}\right\}$, and $C=\left\{\pi^{-}, \pi^{+}\right\}$. Each of the Dalitz variables are divided into five ranges for which the efficiencies are compared. A statistically significant variation is found only in the variable $M\left(\pi^{+} \Lambda_{c}^{+}\right)^{2}$ for the $\Xi_{c}(3077)^{+}$efficiency. The signal-MC samples are binned into three ranges of $M(C A)$ for calculating efficiencies: below $2470 \mathrm{MeV} / c^{2}$, between $2470 \mathrm{MeV} / c^{2}$ and $2550 \mathrm{MeV} / c^{2}$, and above $2550 \mathrm{MeV} / c^{2}$. These ranges are chosen such that the highest invariant-mass range contains the possible efficiency variation. These binned efficiencies are listed in Tables 4.9, 4.10, 4.11, and 4.12 for the $\Xi_{c}(2970)^{0}, \Xi_{c}(3077)^{0}, \Xi_{c}(2970)^{+}$, and $\Xi_{c}(3077)^{+}$signals, respectively. The weighted averages of these binned efficiencies are listed on the right-hand side of these tables. Because the $\Xi_{c}(2970)^{0}, \Xi_{c}(2970)^{+}$, and $\Xi_{c}(3077)^{0}$ efficiencies do not appear to depend on when the decay is in phase space, the efficiency for each bin is averaged together with its error as a weight. For the $\Xi_{c}(3077)^{+}$efficiency, each bin averaged together weighted by its volume of the signal-PDF in the $M_{\Xi_{c}}$ vs. $M_{\Sigma_{c}}$ plane; this is done after the fit to data. The $\Xi_{c}(3055)$ and $\Xi_{c}(3123)$ states are found to decay only through $\Sigma_{c} K$ intermediateresonant states. So that their signal-MC samples better represent the data, the $M\left(\Lambda_{c}^{+} \pi^{+}\right)$invariant mass of the $\Xi_{c}(3055)^{+} \mathrm{MC}$ candidates are required to be within $10 \mathrm{MeV} / c^{2}$ of $2454.02 \mathrm{MeV} / c^{2}$ (the world average $\Sigma_{c}(2455)^{++}$mass), and the $M\left(\Lambda_{c}^{+} \pi^{+}\right)$invariant mass of the $\Xi_{c}(3123)^{+} \mathrm{MC}$ candidates are required to be within $30 \mathrm{MeV} / c^{2}$ of $2518.4 \mathrm{MeV} / c^{2}$ (the world average $\Sigma_{c}(2520)^{++}$mass). Efficiencies for $\Xi_{c}(3055)^{0}$ and $\Xi_{c}(3123)^{0}$ are similarly calculated within $10 \mathrm{MeV} / c^{2}$ and $30 \mathrm{MeV} / c^{2}$ of $M\left(\Lambda_{c}^{+} \pi^{-}\right)=2453.76 \mathrm{MeV} / c^{2}$ and $M\left(\Lambda_{c}^{+} \pi^{-}\right)=2518.0 \mathrm{MeV} / c^{2}$, respectively. The three-body decay mode efficiencies for the $\Xi_{c}(3055)$ and $\Xi_{c}(3123)$ states are listed in Table 4.13. 
Table 4.10: Reconstruction efficiencies, in percent, for $\Xi_{c}(3077)^{0} \rightarrow \Lambda_{c}^{+} K_{s} \pi^{-}$three-body decays. Efficiencies are given for each $\Lambda_{c}^{+}$decay mode and for three $M_{\Sigma_{c}}$ ranges. The efficiencies are listed with statistical errors only. The efficiency for each $\Lambda_{c}^{+}$decay mode averaged over the three $M_{\Sigma_{c}}$ ranges is listed with statistical (first) and systematic (second) errors.

\begin{tabular}{lcccc}
\hline Decay Mode & $<2470 \mathrm{MeV} / c^{2}$ & $(2470-2550) \mathrm{MeV} / c^{2}$ & $>2550 \mathrm{MeV} / c^{2}$ & Average \\
\hline \hline$p K^{-} \pi^{+}$ & $1.62 \pm 0.03$ & $1.59 \pm 0.02$ & $1.64 \pm 0.05$ & $1.59 \pm 0.02 \pm 0.09$ \\
\hline$p K_{s}$ & $0.46 \pm 0.03$ & $0.45 \pm 0.02$ & $0.53 \pm 0.04$ & $0.50 \pm 0.01 \pm 0.04$ \\
\hline$p K_{s} \pi^{+} \pi^{-}$ & $0.19 \pm 0.01$ & $0.18 \pm 0.01$ & $0.16 \pm 0.02$ & $0.20 \pm 0.01 \pm 0.03$ \\
\hline$\Lambda^{0} \pi^{+}$ & $0.85 \pm 0.06$ & $0.78 \pm 0.03$ & $0.93 \pm 0.08$ & $0.91 \pm 0.03 \pm 0.07$ \\
\hline$\Lambda^{0} \pi^{+} \pi^{-} \pi^{+}$ & $0.36 \pm 0.02$ & $0.33 \pm 0.01$ & $0.37 \pm 0.03$ & $0.38 \pm 0.01 \pm 0.03$ \\
\hline \hline
\end{tabular}

Table 4.11: Reconstruction efficiencies, in percent, for $\Xi_{c}(2970)^{+} \rightarrow \Lambda_{c}^{+} K^{-} \pi^{+}$three-body decays. Efficiencies are given for each $\Lambda_{c}^{+}$decay mode and for two $M_{\Sigma_{c}}$ ranges. The efficiencies are listed with statistical errors only. The efficiency for each $\Lambda_{c}^{+}$decay mode averaged over the two $M_{\Sigma_{c}}$ ranges is listed with statistical (first) and systematic (second) errors.

\begin{tabular}{lccc}
\hline Decay Mode & $<2470 \mathrm{MeV} / c^{2}$ & $(2470-2550) \mathrm{MeV} / c^{2}$ & Average \\
\hline \hline$p K^{-} \pi^{+}$ & $8.70 \pm 0.07$ & $8.68 \pm 0.21$ & $8.70 \pm 0.07 \pm 0.50$ \\
\hline$p K_{s}$ & $2.43 \pm 0.05$ & $2.57 \pm 0.16$ & $2.40 \pm 0.05 \pm 0.13$ \\
\hline$p K_{s} \pi^{+} \pi^{-}$ & $1.03 \pm 0.03$ & $1.04 \pm 0.09$ & $1.01 \pm 0.03 \pm 0.09$ \\
\hline$\Lambda^{0} \pi^{+}$ & $3.93 \pm 0.11$ & $4.55 \pm 0.35$ & $3.91 \pm 0.11 \pm 0.23$ \\
\hline$\Lambda^{0} \pi^{+} \pi^{-} \pi^{+}$ & $2.07 \pm 0.04$ & $1.92 \pm 0.12$ & $2.01 \pm 0.04 \pm 0.19$ \\
\hline \hline
\end{tabular}


Table 4.12: Reconstruction efficiencies, in percent, for $\Xi_{c}(3077)^{+} \rightarrow \Lambda_{c}^{+} K^{-} \pi^{+}$three-body decays. Efficiencies are given for each $\Lambda_{c}^{+}$decay mode and for three $M_{\Sigma_{c}}$ ranges. The efficiencies are listed with statistical errors only. The efficiency for each $\Lambda_{c}^{+}$decay mode averaged over the three $M_{\Sigma_{c}}$ ranges is listed with statistical (first) and systematic (second) errors.

\begin{tabular}{lcccc}
\hline Decay Mode & $<2470 \mathrm{MeV} / c^{2}$ & $(2470-2550) \mathrm{MeV} / c^{2}$ & $>2550 \mathrm{MeV} / c^{2}$ & Average \\
\hline \hline$p K^{-} \pi^{+}$ & $8.59 \pm 0.14$ & $8.75 \pm 0.08$ & $9.18 \pm 0.17$ & $8.69 \pm 0.07 \pm 0.50$ \\
\hline$p K_{s}$ & $2.29 \pm 0.10$ & $2.48 \pm 0.07$ & $2.67 \pm 0.13$ & $2.35 \pm 0.06 \pm 0.13$ \\
\hline$p K_{s} \pi^{+} \pi^{-}$ & $0.96 \pm 0.06$ & $0.94 \pm 0.04$ & $1.08 \pm 0.07$ & $0.94 \pm 0.03 \pm 0.08$ \\
\hline$\Lambda^{0} \pi^{+}$ & $4.30 \pm 0.23$ & $4.19 \pm 0.14$ & $4.20 \pm 0.27$ & $4.16 \pm 0.12 \pm 0.25$ \\
\hline$\Lambda^{0} \pi^{+} \pi^{-} \pi^{+}$ & $1.89 \pm 0.08$ & $2.00 \pm 0.05$ & $2.03 \pm 0.10$ & $1.91 \pm 0.04 \pm 0.13$ \\
\hline \hline
\end{tabular}

Table 4.13: Reconstruction efficiencies, in percent, for $\Xi_{c}(3055)^{+, 0}$ and $\Xi_{c}(3123)^{+, 0}$ three-body decays. The $\Xi_{c}(3055)^{+, 0}$ and $\Xi_{c}(3123)^{+, 0}$ efficiencies are for $M_{\Sigma_{c}}$ ranges around the $\Sigma_{c}(2455)^{++, 0}$ and $\Sigma_{c}(2520)^{++, 0}$, respectively. The errors are statistical (first) and systematic (second).

\begin{tabular}{lcccc}
\hline Decay Mode & $\Xi_{c}(3055)^{+}$ & $\Xi_{c}(3123)^{+}$ & $\Xi_{c}(3055)^{0}$ & $\Xi_{c}(3123)^{0}$ \\
\hline \hline$p K^{-} \pi^{+}$ & $8.91 \pm 0.16 \pm 0.59$ & $8.87 \pm 0.10 \pm 0.51$ & $1.70 \pm 0.04 \pm 0.13$ & $1.78 \pm 0.03 \pm 0.12$ \\
\hline$p K_{s}$ & $2.54 \pm 0.12 \pm 0.19$ & $2.50 \pm 0.08 \pm 0.14$ & $0.59 \pm 0.04 \pm 0.06$ & $0.56 \pm 0.02 \pm 0.05$ \\
\hline$p K_{s} \pi^{+} \pi^{-}$ & $1.08 \pm 0.07 \pm 0.10$ & $0.96 \pm 0.04 \pm 0.07$ & $0.20 \pm 0.02 \pm 0.03$ & $0.22 \pm 0.01 \pm 0.02$ \\
\hline$\Lambda^{0} \pi^{+}$ & $4.21 \pm 0.25 \pm 0.30$ & $4.67 \pm 0.17 \pm 0.29$ & $0.98 \pm 0.08 \pm 0.08$ & $1.00 \pm 0.05 \pm 0.08$ \\
\hline$\Lambda^{0} \pi^{+} \pi^{-} \pi^{+}$ & $2.19 \pm 0.10 \pm 0.19$ & $1.99 \pm 0.06 \pm 0.16$ & $0.44 \pm 0.03 \pm 0.05$ & $0.41 \pm 0.02 \pm 0.04$ \\
\hline \hline
\end{tabular}




\subsubsection{Two-Dimensional PDF Components}

The two-dimensional PDFs that are used to describe the distribution of $M_{\Xi_{c}}$ versus $M_{\Sigma_{c}}$ data have components that describe nonresonant combinatoric background, resonant combinatoric background, nonresonant signal, and resonant signal. The PDF components are tested with fits to various background data samples and MC samples. Candidates for all $\Lambda_{c}^{+}$decay modes are fit simultaneously using PDF shape parameters that are all shared between the subsamples.

Nonresonant Combinatoric Background: One PDF component is used to fit for nonresonant combinatoric background (background with no $\Sigma_{c}(2455)^{0,++}$ or $\Sigma_{c}(2520)^{0,++}$ resonances). $\Lambda_{c}^{+} K_{s} \pi^{-}$ candidates for which the $\Lambda_{c}^{+}$candidate is from a $M_{\Lambda_{c}}$ sideband region are used to illustrate and test this PDF component. The $\Lambda_{c}^{+}$mass sidebands are in the approximate range $3.5 \sigma<\mid M_{\Lambda_{c}}-$ $2286.46 \mathrm{MeV} / c^{2} \mid<7.0 \sigma$, where $\sigma$ is the weighted average of the two Gaussian widths of the $\Lambda_{c}^{+}$ peak. The $\Lambda_{c}^{+}$mass sideband regions are illustrated in Figure 4.5. The PDF component used to fit these sideband data is proportional to a threshold function $T\left(M_{\Xi_{c}}\right)$ in $M_{\Xi_{c}}$ and a threshold function $T\left(M_{\Sigma_{c}}\right)$ in $M_{\Sigma_{c}}$. These threshold functions are of the form

$$
T(M)=M\left[\left(\frac{M}{t}\right)^{2}-1\right]^{\alpha} \exp \left[\beta\left(\left(\frac{M}{t}\right)^{2}-1\right)\right],
$$

where $M$ is the mass variable in which there is a minimum kinematic threshold $t$, and $\alpha$ and $\beta$ are parameters that determine the shape of the function. The parameters $\alpha$ and $\beta$ are shared between $T\left(M_{\Xi_{c}}\right)$ and $T\left(M_{\Sigma_{c}}\right)$. For $T\left(M_{\Sigma_{c}}\right)$, the threshold $t$ is a constant $2426.02 \mathrm{MeV} / c^{2}$. For $T\left(M_{\Xi_{c}}\right)$, the threshold is dependent on $M_{\Sigma_{c}}$ through the relation $t=M_{\Sigma_{c}}+497.648 \mathrm{MeV} / c^{2}$. The $\Lambda_{c}^{+} K_{s} \pi^{-}$data that have a $\Lambda_{c}^{+}$candidate in the $M_{\Lambda_{c}}$ sideband region are shown in Figures 4.6 (projections in $M_{\Xi_{c}}$ ) and 4.7 (projections in $M_{\Sigma_{c}}$ ). The fit to these data with the two-dimensional background PDF component just described is also shown. The data and PDF projections in Figures 4.6 and 4.7 show that the two-dimensional PDF component used to fit nonresonant combinatoric background has an appropriate shape. They also demonstrate that, despite possible differences in the $\Lambda_{c}^{+}$combinatoric backgrounds, the background shape parameters can be shared between the different $\Lambda_{c}^{+}$decay modes.

Resonant Combinatoric Background: Another PDF component is used to fit $\Sigma_{c}(2455)^{0,++}$ and $\Sigma_{c}(2520)^{0,++}$ resonant combinatoric background. This is background with real $\Sigma_{c}(2455)^{0,++}$ and $\Sigma_{c}(2520)^{0,++}$ resonances that peak in the $M_{\Sigma_{c}}$ invariant-mass distribution, but the full three body candidate is a combination of this resonance and a random track from the rest of the event that is not from an excited charm-strange baryon decay. A wrong-sign $\Lambda_{c}^{+} K^{+} \pi^{-}$data set is used to illustrate and test this PDF component. The resonant-background PDF component is proportional to the product of a threshold function $T\left(M_{\Xi_{c}}\right)$, a double-Voigtian function $\mathcal{V}\left(M_{\Sigma_{c}}\right)$, and a two-body phase-space function $F\left(M_{\Sigma_{c}}\right)$. The threshold function is the same as that given in by Equation 4.8 and shares the same $\alpha$ and $\beta$ parameters as the nonresonant background PDF component. The double-Voigtian function $\mathcal{V}\left(M_{\Sigma_{c}}\right)$ is a nonrelativistic Breit-Wigner shape convolved with 

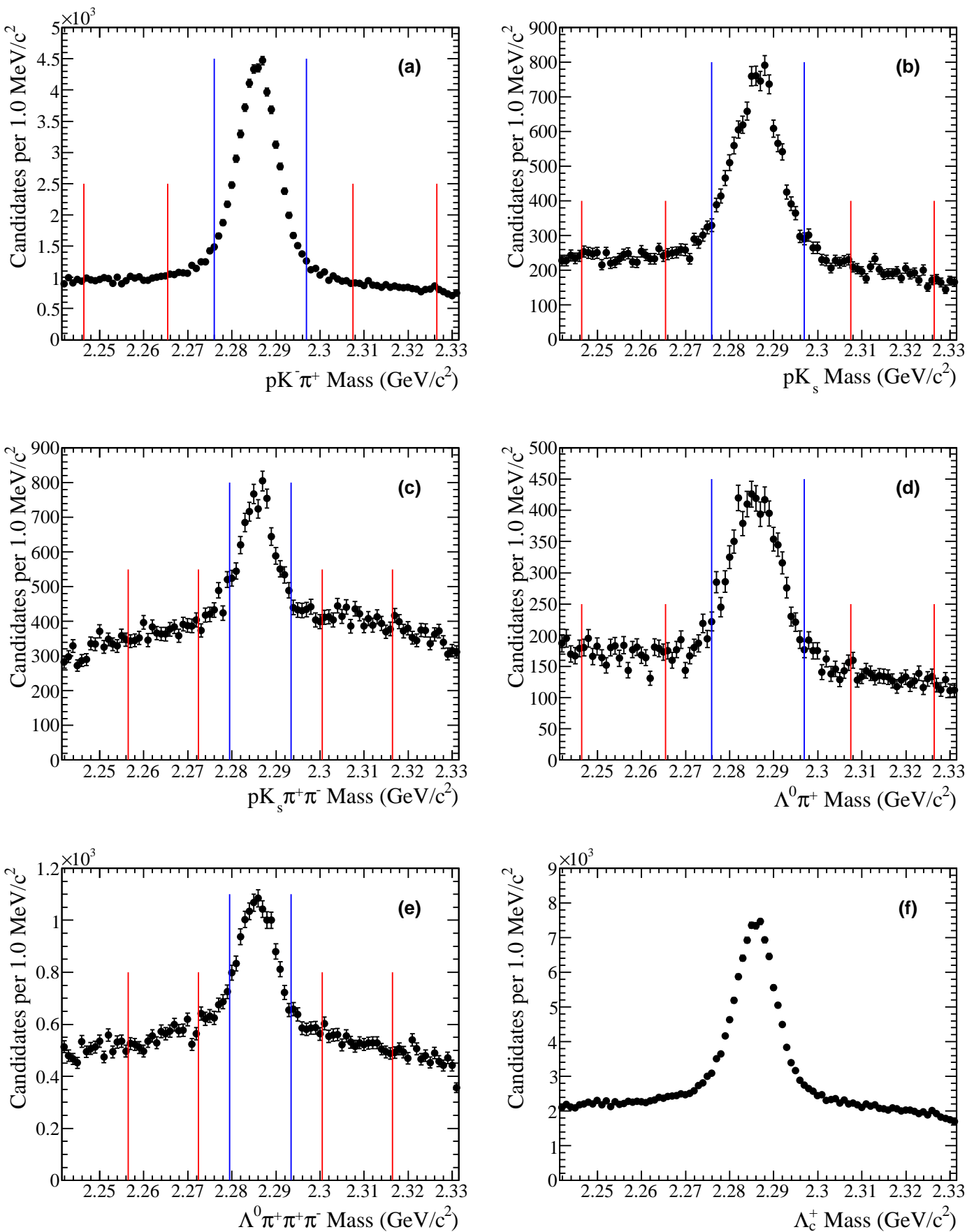

Figure 4.5: Reconstructed invariant-mass distributions for $\Lambda_{c}^{+}$candidates in data (points with error bars). The blue lines indicate the mass regions used for the $\Lambda_{c}^{+}$signal regions. The red lines indicate the mass regions used for $\Lambda_{c}^{+}$sideband regions. The $\Lambda_{c}^{+}$is reconstructed in decays to (a) $p K^{-} \pi^{+}$, (b) $p K_{s}$, (c) $p K_{s} \pi^{-} \pi^{+}$, (d) $\Lambda^{0} \pi^{+}$, (e) $\Lambda^{0} \pi^{+} \pi^{-} \pi^{+}$, and (f) all five final states. 

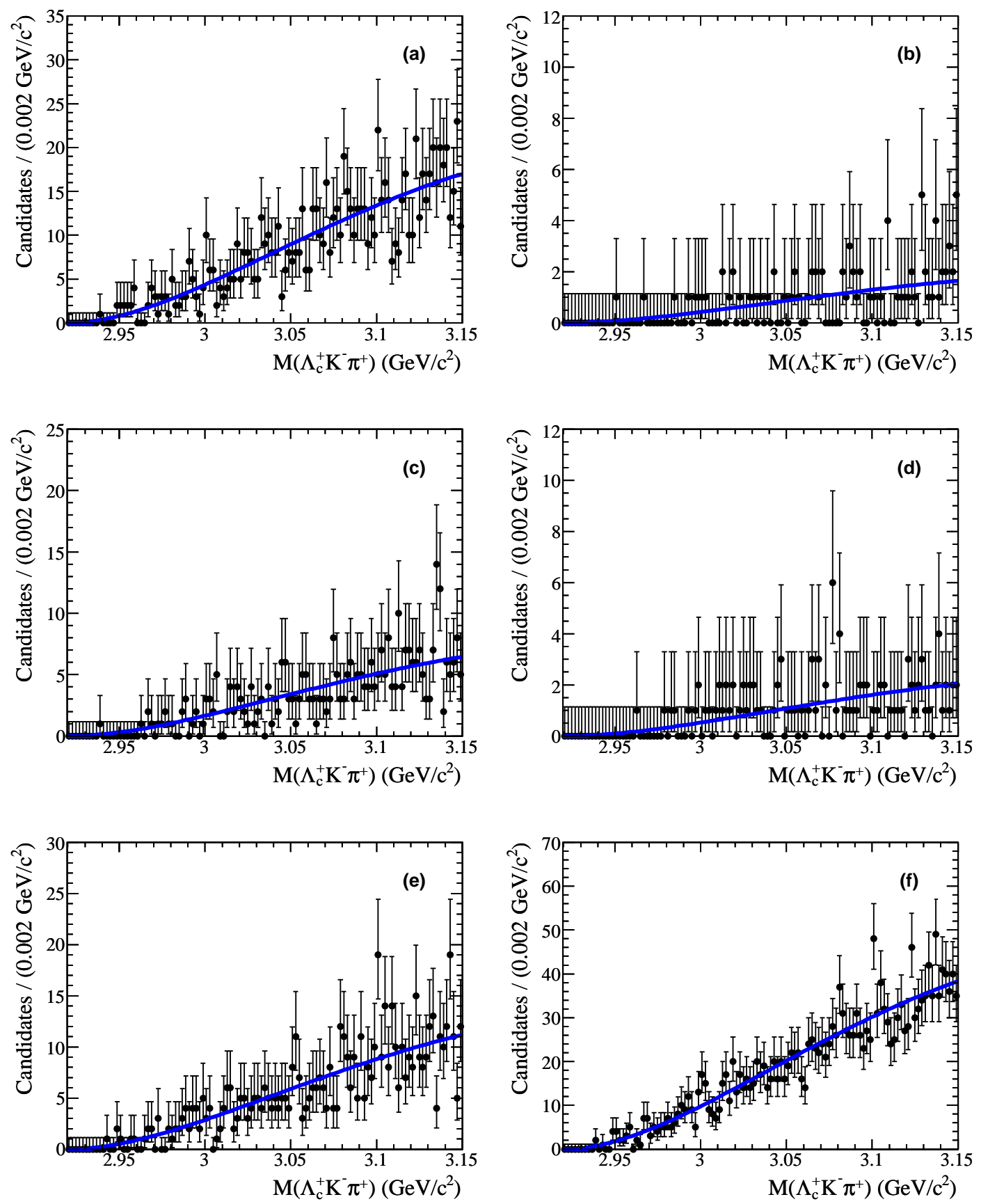

Figure 4.6: $M_{\Xi_{c}}$ projections of $\Lambda_{c}^{+} K_{s} \pi^{-}$data for which the $\Lambda_{c}^{+}$candidate is from a $M_{\Lambda_{c}}$ sideband region (points with error bars) fit with a threshold function (blue curves) that describes nonresonant combinatoric background. The $\Lambda_{c}^{+}$candidates are reconstructed in decays to (a) $p K^{-} \pi^{+}$, (b) $p K_{s}$, (c) $p K_{s} \pi^{-} \pi^{+}$, (d) $\Lambda^{0} \pi^{+}$, (e) $\Lambda^{0} \pi^{+} \pi^{-} \pi^{+}$, and (f) all five final states. The corresponding $M_{\Sigma_{c}}$ projections are shown in Figure 4.7. 

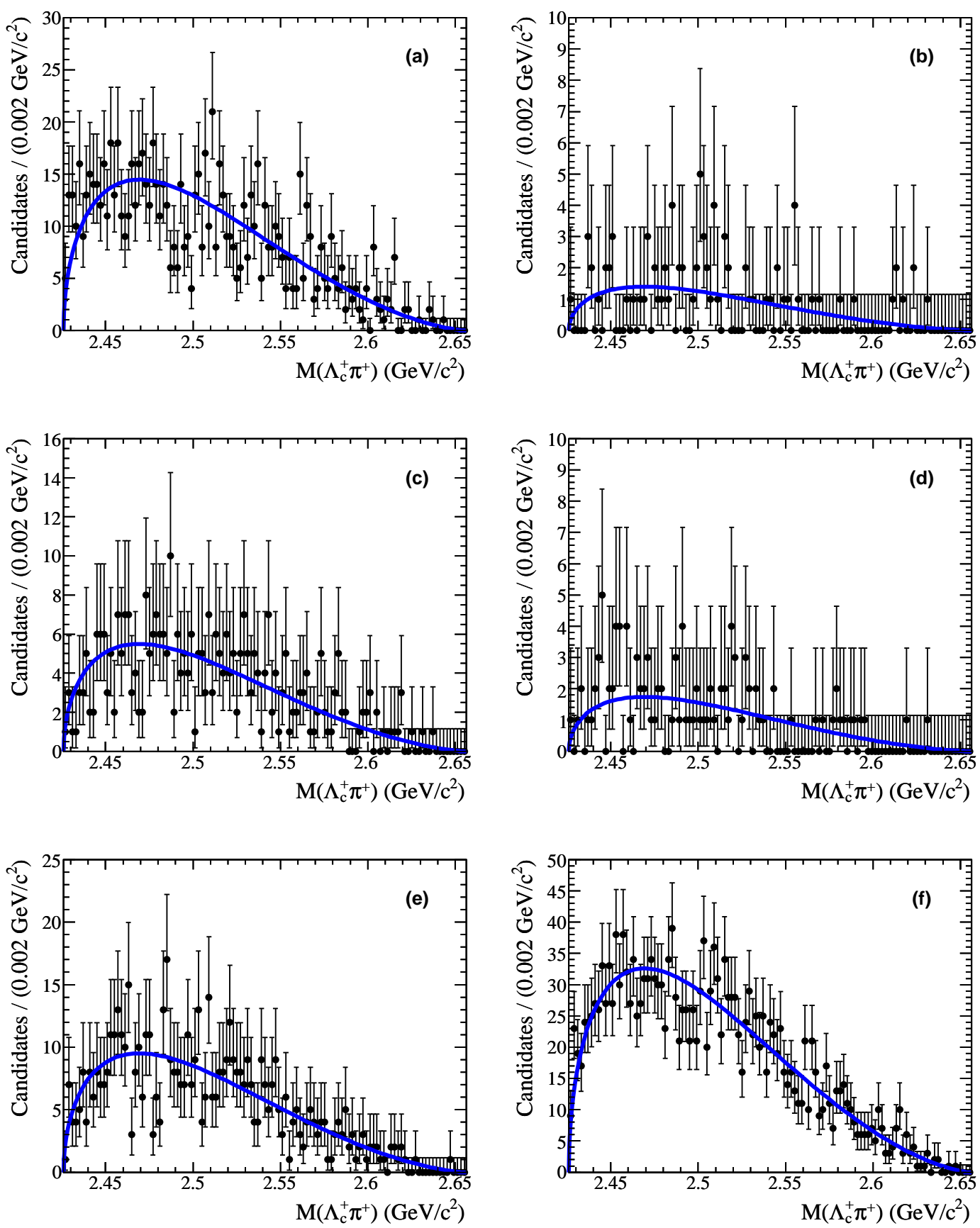

Figure 4.7: $M_{\Sigma_{c}}$ projections of $\Lambda_{c}^{+}$-sideband $\Lambda_{c}^{+} K_{s} \pi^{-}$data (points with error bars) fit with nonresonant combinatoric background PDF component (blue curves). The $\Lambda_{c}^{+}$candidates are reconstructed from (a) $p K^{-} \pi^{+}$, (b) $p K_{s}$, (c) $p K_{s} \pi^{-} \pi^{+}$, (d) $\Lambda^{0} \pi^{+}$, (e) $\Lambda^{0} \pi^{+} \pi^{-} \pi^{+}$, and (f) all five final states. The corresponding $M_{\Xi_{c}}$ projections are shown in Figure 4.6. 
two Gaussian resolution functions:

$$
\begin{gathered}
\mathcal{V}(M)=\left[\frac{1}{\left(M-M_{0}\right)^{2}+\Gamma^{2} / 4}\right] \otimes\left[f \exp \left(-\frac{\left(x-\mu_{1}\right)^{2}}{2 \sigma_{1}^{2}}\right)+(1-f) \exp \left(-\frac{\left(x-\mu_{2}\right)^{2}}{2 \sigma_{2}^{2}}\right)\right] \\
=f V_{1}\left(M ; M_{0}, \Gamma, \sigma_{1}, \mu_{1}\right)+(1-f) V_{2}\left(M ; M_{0}, \Gamma, \sigma_{2}, \mu_{2}\right)
\end{gathered}
$$

where $M, M_{0}$, and $\Gamma$ are respectively the mass variable, the pole mass, and the width of a BreitWigner, and $\mu_{1}, \mu_{2}$ and $\sigma_{1}, \sigma_{2}$ are the means and widths of the two Gaussian resolution functions with relative fractions $f$ and $1-f$. For the $\Lambda_{c}^{+} K^{-} \pi^{+}$candidates, the parameters $M_{0}$ and $\Gamma$ for the $\Sigma_{c}^{++}$resonances are free parameters in the fit. For the $\Lambda_{c}^{+} K_{s} \pi^{-}$candidates, the parameters $M_{0}$ and $\Gamma$ are fixed to world average values for the $\Sigma_{c}^{0}$ resonances [7]. These parameters are fixed because they are not a final result of this study and the $\Sigma_{c}^{0}$ resonances have a small number of peaking candidates making a fit of these parameters difficult. For three-body decay PDF components, the parameters $\mu_{1}$ and $\mu_{2}$ are always fixed at zero. The $\sigma_{1}, \sigma_{2}$, and $f$ parameters have the same values for each $\Lambda_{c}^{+}$ data subsample and are the average of the values listed in Tables 4.7 and 4.8. The resultant terms $V_{1}$ and $V_{2}$ in Equation 4.9 are known as Voigtian line shapes. Two of these resonant-background PDF components are used to fit the $\Sigma_{c}(2455)^{0,+}$ and $\Sigma_{c}(2520)^{0,+}$ background resonances. The two-body phase-space function is

$$
F\left(M_{\Sigma_{c}}\right)=\frac{\left[\left(M_{\Sigma_{c}}^{2}-\left(m_{\Lambda_{c}}+m_{\pi}\right)^{2}\right)\left(M_{\Sigma_{c}}^{2}-\left(m_{\Lambda_{c}}-m_{\pi}\right)^{2}\right)\right]^{1 / 2}}{2 M_{\Sigma_{c}}}
$$

where $m_{\Lambda_{c}}$ is the world average $\Lambda_{c}^{+}$mass and $m_{\pi}$ is the world average $\pi^{+}$mass [7]. The wrongsign $\Lambda_{c}^{+} K^{+} \pi^{-}$data set, and the nonresonant and resonant two-dimensional PDF fit are shown in Figures 4.8 (projections in $M_{\Xi_{c}}$ ) and 4.9 (projections in $M_{\Sigma_{c}}$ ).

Resonant and Nonresonant Signal: Two PDF components are used to describe the signal. One component describes nonresonant decays; the other describes two-body resonant decays.

- The nonresonant PDF component is a double-Voigtian function of $M_{\Xi_{c}}, \mathcal{V}\left(M_{\Xi_{c}}\right)$ (Equation 4.9), multiplied by a three-body phase-space function $F\left(M_{\Xi_{c}}, M_{\Sigma_{c}}\right)$ :

$$
F\left(M_{\Xi_{c}}, M_{\Sigma_{c}}\right)=\frac{\left[\left(M_{\Xi_{c}}^{2}-\left(M_{\Sigma_{c}}+m_{K}\right)^{2}\right)\left(M_{\Xi_{c}}^{2}-\left(M_{\Sigma_{c}}-m_{K}\right)^{2}\right)\right]^{1 / 2}}{2 M_{\Xi_{c}}} \times F\left(M_{\Sigma_{c}}\right),
$$

where $m_{K}$ is the world average $K^{+}$or $K_{s}$ mass and $F\left(M_{\Sigma_{c}}\right)$ is the two-body phase space function given in Equation 4.10.

- The resonant-signal PDF components have the form $\mathcal{V}\left(M_{\Xi_{c}}\right) \times F\left(M_{\Xi_{c}}, M_{\Sigma_{c}}\right) \times \mathcal{V}\left(M_{\Sigma_{c}}\right)$ with a double-Voigtian function in $M_{\Sigma_{c}}$ to account for the $\Sigma_{c}(2455)^{++, 0}$ or $\Sigma_{c}(2520)^{++, 0}$ intermediate resonances. The mean and width parameters of the $\Sigma_{c}$ resonances in $\mathcal{V}\left(M_{\Sigma_{c}}\right)$ are shared between the resonant-background PDF and resonant-signal PDF components. 

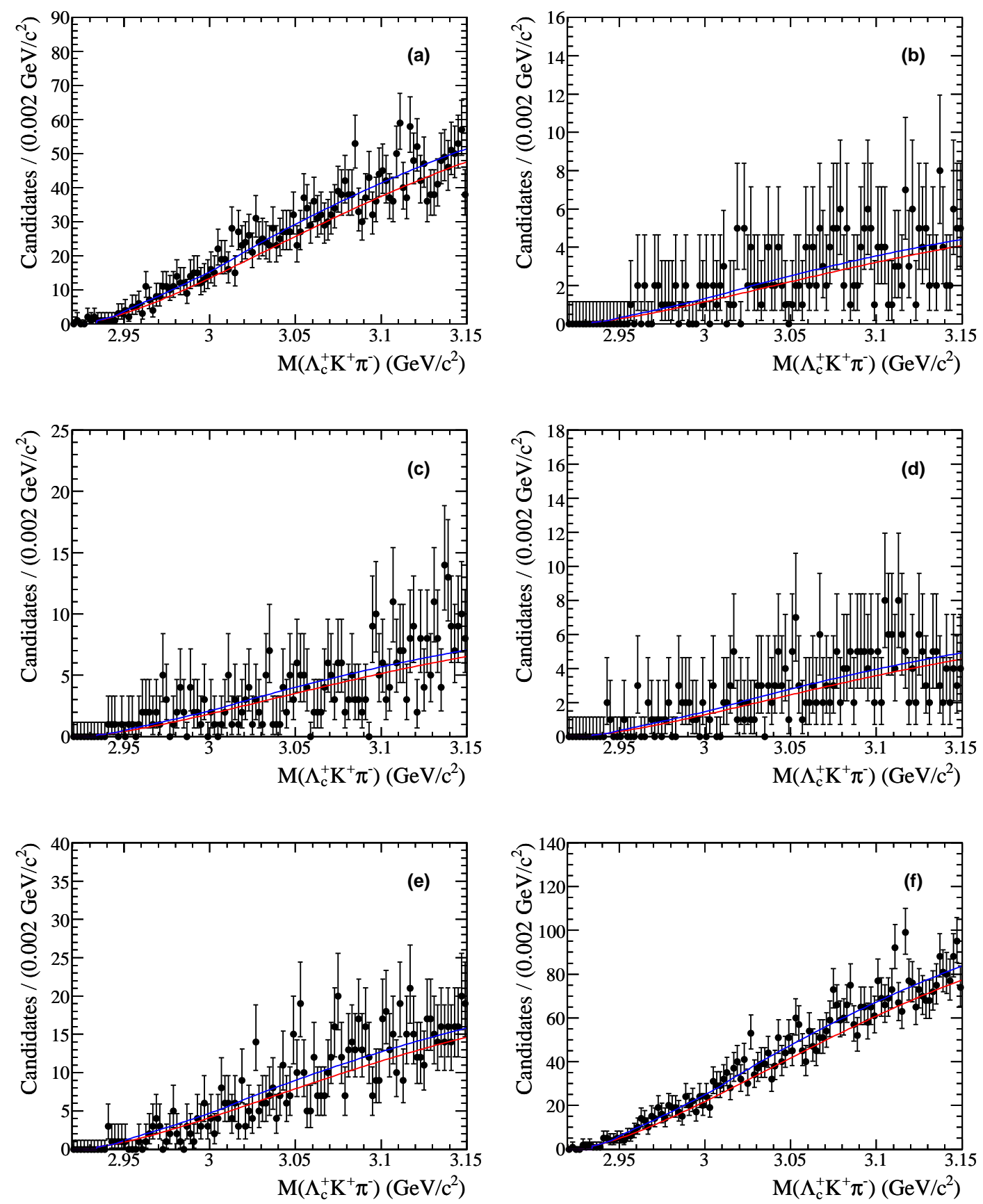

Figure 4.8: $M_{\Xi_{c}}$ projections of wrong-sign $\Lambda_{c}^{+} K^{+} \pi^{-}$candidates in data (points with error bars) fit with the sum of nonresonant and resonant PDF background components. The blue curves represent the total PDF while the red curves represent the nonresonant component of the PDF. The $\Lambda_{c}^{+}$ candidates are reconstructed from (a) $p K^{-} \pi^{+}$, (b) $p K_{s}$, (c) $p K_{s} \pi^{-} \pi^{+}$, (d) $\Lambda^{0} \pi^{+}$, (e) $\Lambda^{0} \pi^{+} \pi^{-} \pi^{+}$, and (f) all five final states. The corresponding $M_{\Sigma_{c}}$ projections are shown in Figure 4.9. 

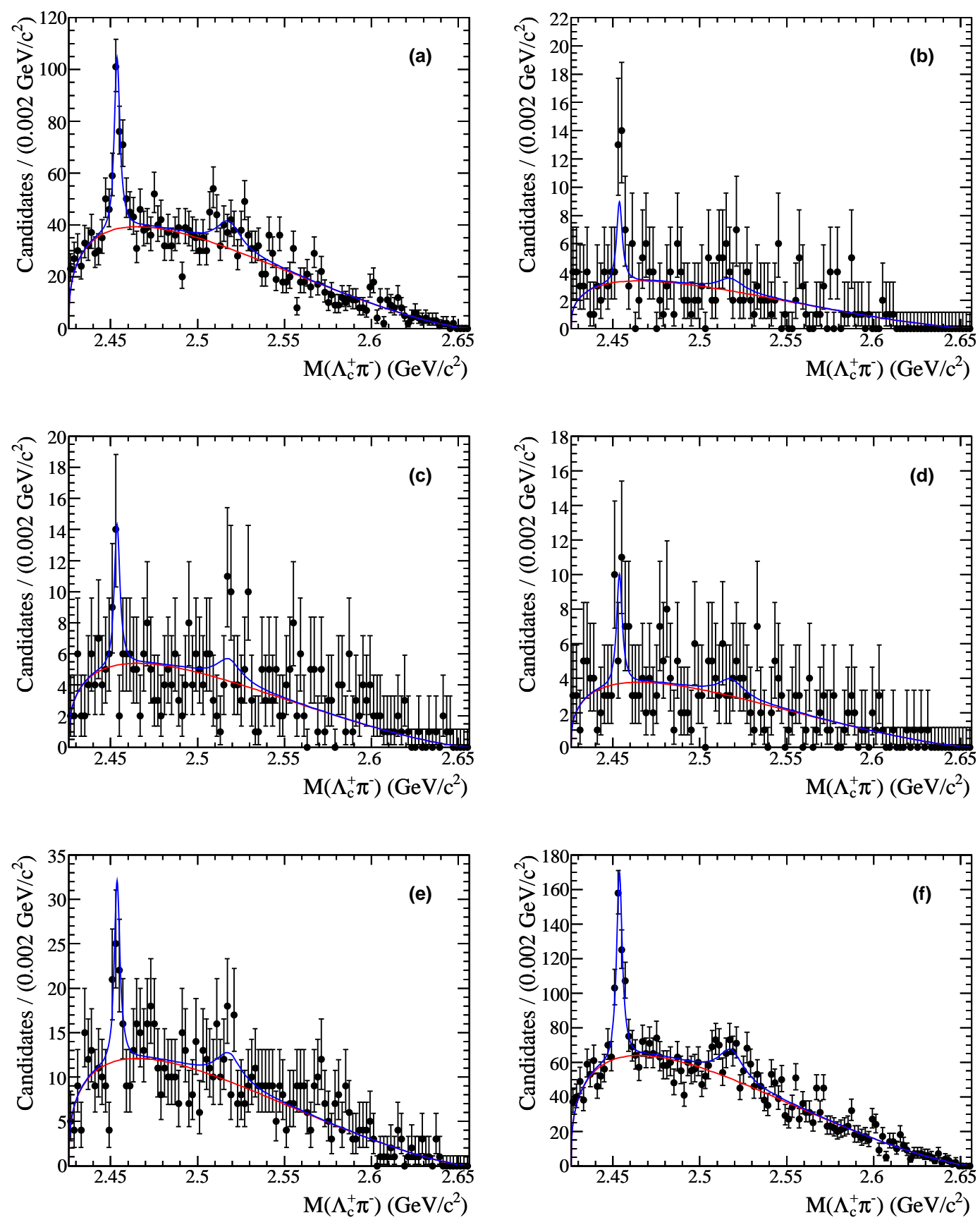

Figure 4.9: $M_{\Sigma_{c}}$ projections of wrong-sign $\Lambda_{c}^{+} K^{+} \pi^{-}$candidates in data (points with error bars) fit with the sum of nonresonant and resonant PDF background components. The blue curves represent the total PDF while the red curves represent the nonresonant component of the PDF. The $\Lambda_{c}^{+}$ candidates are reconstructed from (a) $p K^{-} \pi^{+}$, (b) $p K_{s}$, (c) $p K_{s} \pi^{-} \pi^{+}$, (d) $\Lambda^{0} \pi^{+}$, (e) $\Lambda^{0} \pi^{+} \pi^{-} \pi^{+}$, and (f) all five final states. The corresponding $M_{\Xi_{c}}$ projections are shown in Figure 4.8. 
The mass resolution parameters in $\mathcal{V}\left(M_{\Xi_{c}}\right)$ and $\mathcal{V}\left(M_{\Sigma_{c}}\right)$ for both the nonresonant and the resonant PDF components are those listed in Tables 4.6, 4.7, and 4.8.

I analyze a background sample of MC events that simulate generic types of $e^{+} e^{-} \rightarrow c \bar{c}$ events that do not have any excited charm-strange baryons, and I reconstruct $\Lambda_{c}^{+} K^{-} \pi^{+}$and $\Lambda_{c}^{+} K_{s} \pi^{-}$signal candidates from the background $\mathrm{MC}$ sample. This sample of generic- $c \bar{c} \mathrm{MC}$ candidates is used to represent about $380 \mathrm{fb}^{-1}$ worth of $e^{+} e^{-} \rightarrow c \bar{c}$ data, with no excited charm-strange baryon signals. I combine the generic- $c \bar{c}$ MC sample of $\Lambda_{c}^{+} K_{s} \pi^{-}$candidates with a TMC signal distributions based on the nonresonant PDF components for $\Xi_{c}(2970)^{0}$ and $\Xi_{c}(3077)^{0}$, and I combine the generic-c $\bar{c}$ MC sample of $\Lambda_{c}^{+} K^{-} \pi^{+}$candidates with a TMC signal distributions based on the resonant PDF components for $\Xi_{c}(2970)^{+}$and $\Xi_{c}(3077)^{+}$. The resonant TMC distribution represents $\Xi_{c}(2970)^{+}$ signal decaying through the intermediate-resonant state $\Sigma_{c}(2455)^{++} K^{-}$and a $\Xi_{c}(3077)^{+}$signal decaying through the intermediate-resonant states $\Sigma_{c}(2455)^{++} K^{-}$and $\Sigma_{c}(2520)^{++} K^{-}$. The combined MC and TMC samples are used to test PDF parameterization with both background and signal components together. The combined MC and TMC samples for $\Lambda_{c}^{+} K_{s} \pi^{-}$are illustrated in Figures 4.10 (projections in $M_{\Xi_{c}}$ ) and 4.11 (projections in $M_{\Sigma_{c}}$ ). The combined MC and TMC samples for $\Lambda_{c}^{+} K^{-} \pi^{+}$are illustrated in Figures 4.12 (projections in $M_{\Xi_{c}}$ ) and 4.13 (projections in $M_{\Sigma_{c}}$ ). These combined samples are each fit with a PDF consisting of components corresponding to the generic- $c \bar{c}$ sample and components corresponding to the TMC.

All PDF shape parameters are shared between the different $\Lambda_{c}^{+}$decay modes for the simultaneous fit; only the fitted numbers of candidates are independent between the $\Lambda_{c}^{+}$decay modes. The resonant and nonresonant signal-PDF components are combined, using a parameter for the fraction of each, into a single PDF component for $\Xi_{c}(2970)^{+, 0}$. A single PDF component for $\Xi_{c}(3077)^{+, 0}$ is created by combining, with fraction parameters, two intermediate-resonant signal components (for $\Sigma_{c}(2455) K$ and $\left.\Sigma_{c}(2520) K\right)$ and a nonresonant signal component. These fractions are shared between the different $\Lambda_{c}^{+}$decay modes. The signal PDF components for $\Xi_{c}(3055)^{+, 0}$ and $\Xi_{c}(3123)^{+, 0}$ describe only intermediate-resonant $\Sigma_{c}(2455) K$ and $\Sigma_{c}(2520) K$ decays, respectively.

\subsubsection{Likelihood Function}

The likelihood function used to fit the data is extended to allow Poisson fluctuations for the number of fitted charm-strange baryon signal candidates for each of the $\Lambda_{c}^{+}$decay modes. The likelihood function also provides Poisson errors for the combined number of background candidates from each background component of the PDF. The numbers of signal candidates are allowed to be negative. The PDFs used to construct all likelihood functions are consistently normalized to provide probabilities.

For each $\Lambda_{c}^{+}$decay mode $m$, the corresponding portion of the likelihood function $L_{m}$ has the 

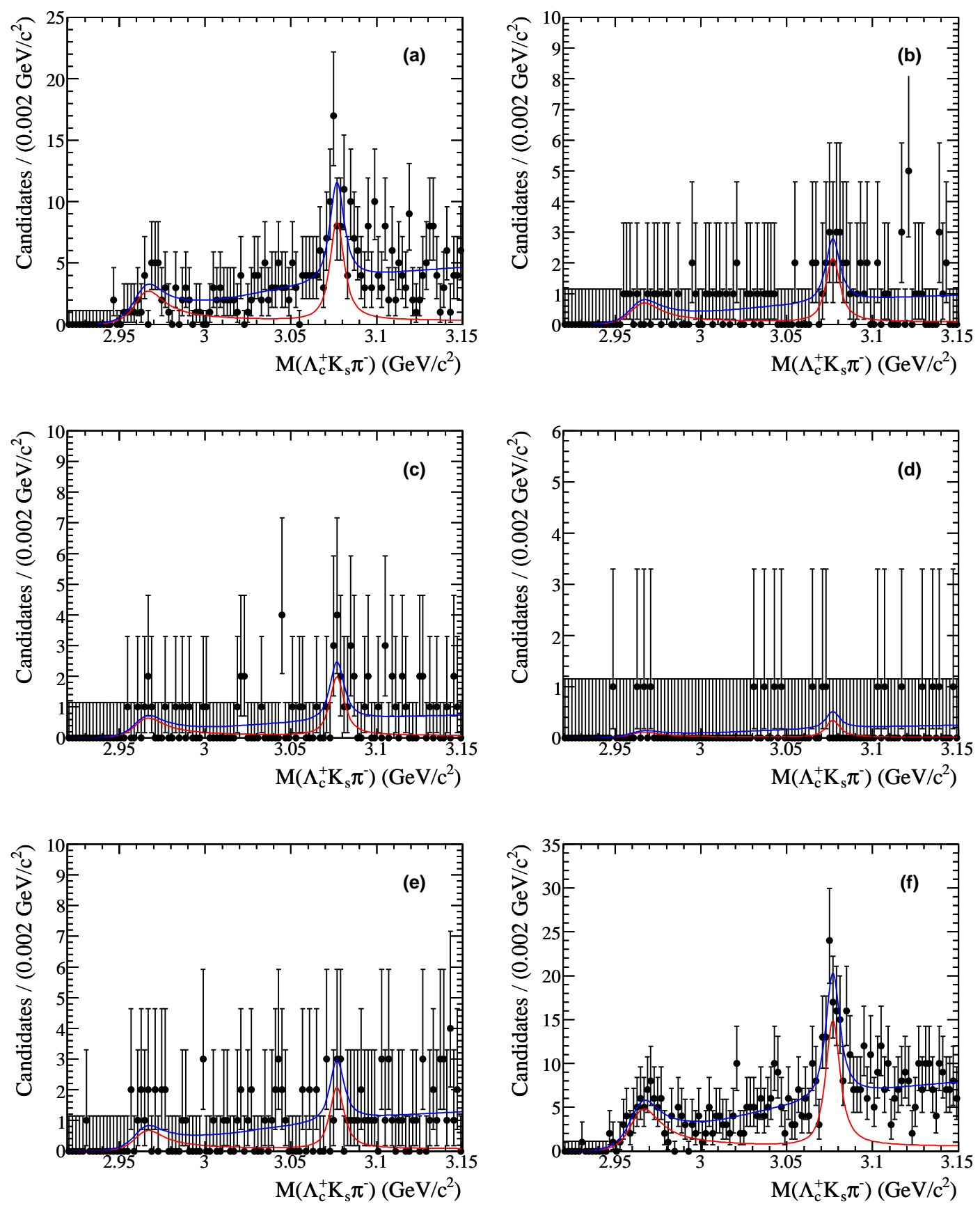

Figure 4.10: $M_{\Xi_{c}}$ projections for generic- $c \bar{c} \Lambda_{c}^{+} K_{s} \pi^{-}$MC candidates combined with nonresonant signal TMC (points with error bars), and an illustration of the nonresonant signal PDF components. The blue curves represent the total PDF while the red curves represent the background components of the PDF. The $\Lambda_{c}^{+}$candidates are reconstructed from (a) $p K^{-} \pi^{+}$, (b) $p K_{s}$, (c) $p K_{s} \pi^{-} \pi^{+}$, (d) $\Lambda^{0} \pi^{+}$, (e) $\Lambda^{0} \pi^{+} \pi^{-} \pi^{+}$, and (f) all five final states. The corresponding $M_{\Sigma_{c}}$ projections are shown in Figure 4.11. 

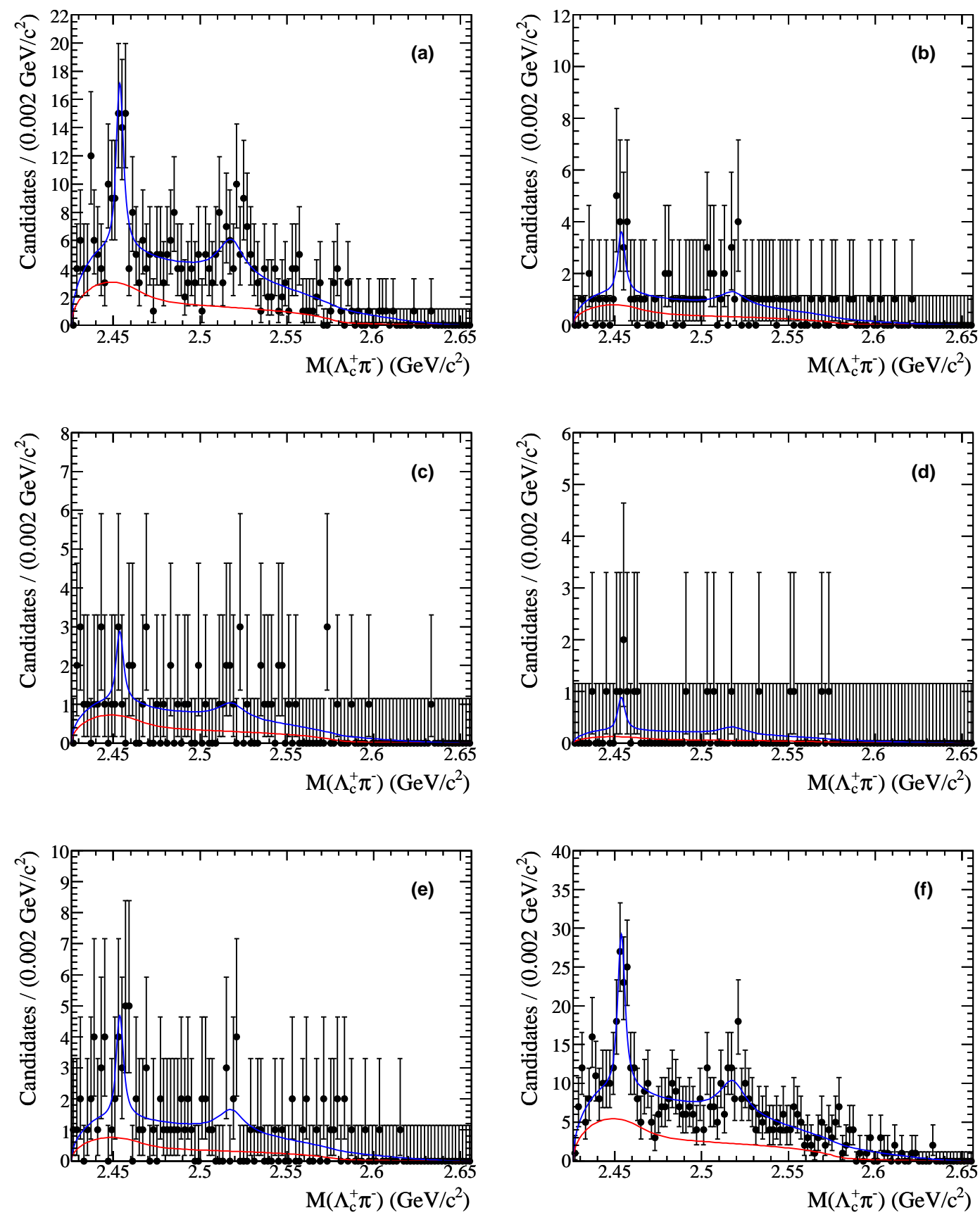

Figure 4.11: $M_{\Sigma_{c}}$ projections for generic-c $\bar{c} \Lambda_{c}^{+} K_{s} \pi^{-} \mathrm{MC}$ candidates combined with signal TMC (points with error bars), and an illustration of the nonresonant signal PDF components. The blue curves represent the total PDF while the red curves represent the background components of the PDF. The $\Lambda_{c}^{+}$candidates are reconstructed from (a) $p K^{-} \pi^{+}$, (b) $p K_{s}$, (c) $p K_{s} \pi^{-} \pi^{+}$, (d) $\Lambda^{0} \pi^{+}$, (e) $\Lambda^{0} \pi^{+} \pi^{-} \pi^{+}$, and (f) all five final states. The corresponding $M_{\Xi_{c}}$ projections are shown in Figure 4.10. 

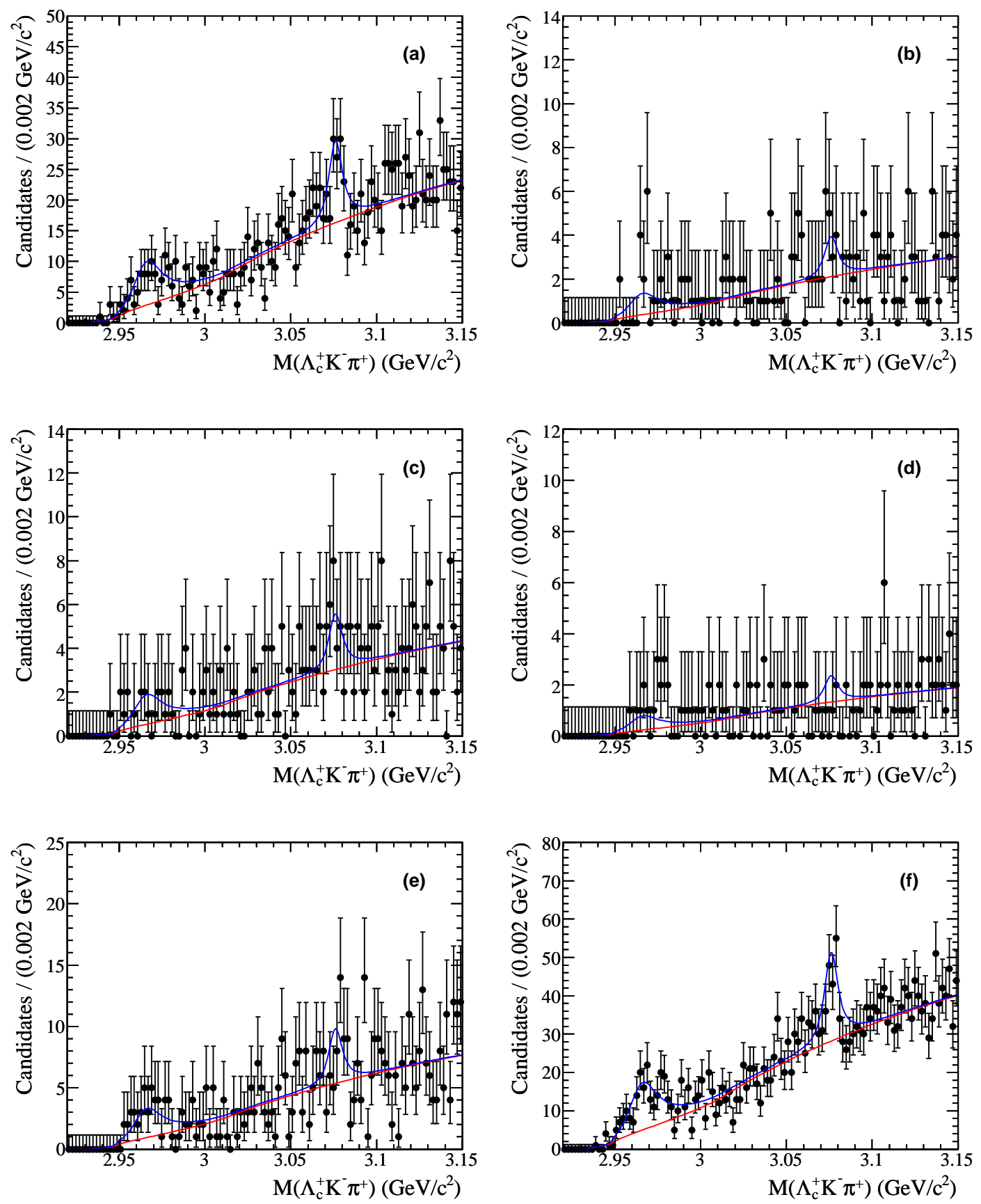

Figure 4.12: $M_{\Xi_{c}}$ projections for generic- $c \bar{c} \Lambda_{c}^{+} K^{-} \pi^{+} \mathrm{MC}$ candidates combined with signal TMC (points with error bars), and an illustration of the resonant signal PDF components. The blue curves represent the total PDF while the red curves represent the signal components of the PDF. The $\Lambda_{c}^{+}$ candidates are reconstructed from (a) $p K^{-} \pi^{+}$, (b) $p K_{s}$, (c) $p K_{s} \pi^{-} \pi^{+}$, (d) $\Lambda^{0} \pi^{+}$, (e) $\Lambda^{0} \pi^{+} \pi^{-} \pi^{+}$, and (f) all five final states. The corresponding $M_{\Sigma_{c}}$ projections are shown in Figure 4.13. 

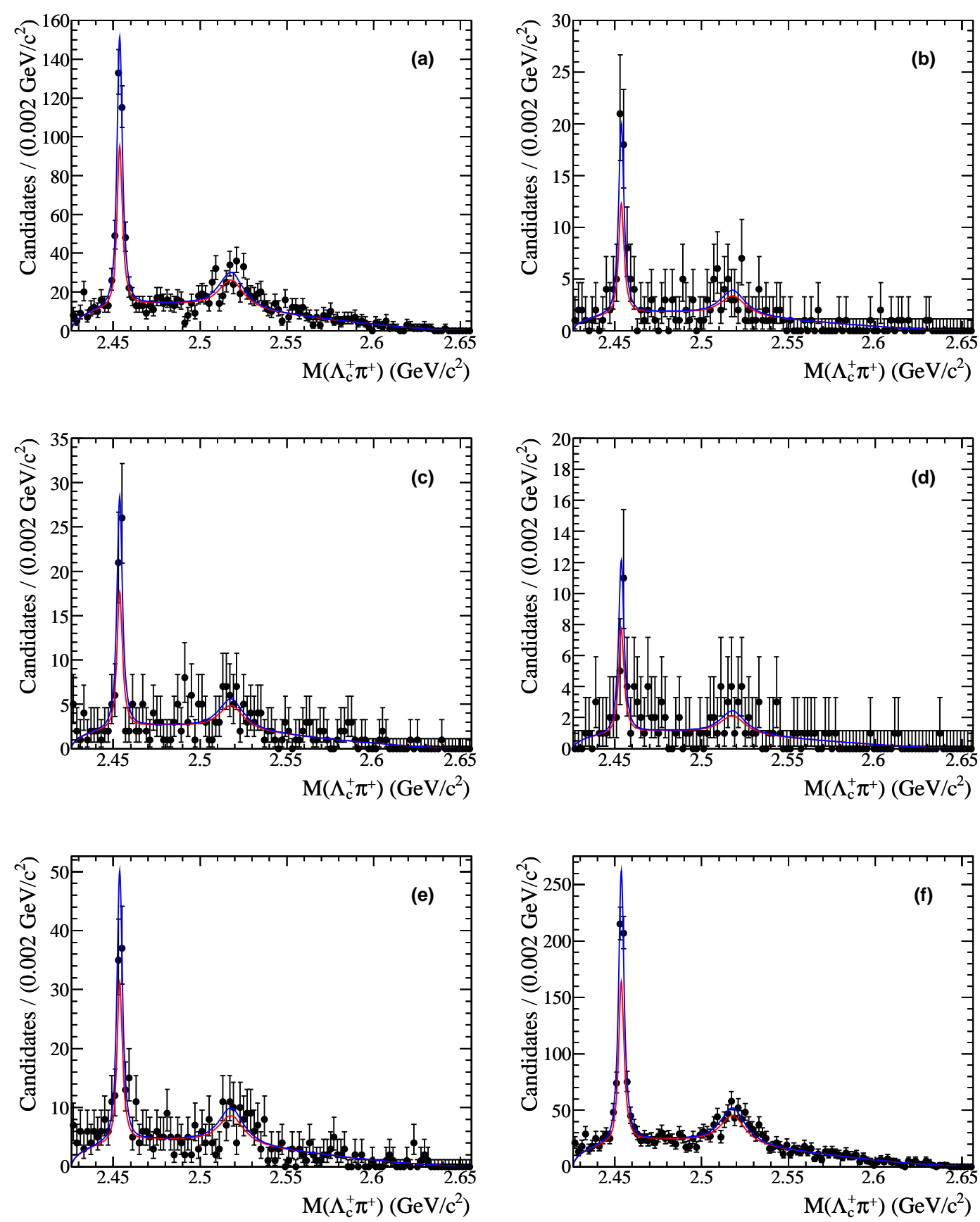

Figure 4.13: $M_{\Sigma_{c}}$ projections for generic-c $\bar{c} \Lambda_{c}^{+} K^{-} \pi^{+}$MC candidates combined with signal TMC (points with error bars), and an illustration of the resonant signal PDF components. The blue curves represent the total PDF while the red curves represent the signal components of the PDF. The $\Lambda_{c}^{+}$ candidates are reconstructed from (a) $p K^{-} \pi^{+}$, (b) $p K_{s}$, (c) $p K_{s} \pi^{-} \pi^{+}$, (d) $\Lambda^{0} \pi^{+}$, (e) $\Lambda^{0} \pi^{+} \pi^{-} \pi^{+}$, and (f) all five final states. The corresponding $M_{\Xi_{c}}$ projections are shown in Figure 4.12. 
form,

$$
L_{m}=\prod_{i}^{N_{m}}\left[\sum_{\eta=1}^{4} S_{m}^{\eta} P_{s}^{\eta}\left(\vec{a} ; x_{m}^{i}\right)+\sum_{\kappa=1}^{3} B_{m}^{\kappa} P_{b}^{\kappa}\left(\vec{b} ; x_{m}^{i}\right)\right],
$$

where $S_{m}^{\eta}$ are the fitted numbers of charm-strange baryon candidates from the four different signalPDF components, $B_{m}^{\kappa}$ are the fitted numbers of non-resonant, $\Sigma_{c}(2455)^{++, 0}$ resonant, and $\Sigma_{c}(2520)^{++, 0}$ resonant background candidates from the three different background-PDF components, $\vec{a}$ and $\vec{b}$ are the shape parameters for the signal-PDF components and the background-PDF components, $x_{m}$ are the $N_{m}$ measured data points for decay mode $m, P_{s}^{\eta}$ are the normalized signal-PDF components, and $P_{b}^{\kappa}$ are the normalized background-PDF components. The full likelihood function $\mathcal{L}$ has the form

$$
\mathcal{L}=\exp \left[-\sum_{m=1}^{5}\left(N_{m}-\sum_{\eta} S_{m}^{\eta}-\sum_{\kappa} B_{m}^{\kappa}\right)\right] \times \prod_{m=1}^{5} L_{m}
$$

This likelihood function is used to perform a simultaneous unbinned fit of the five $\Lambda_{c}^{+}$decay-mode data subsamples.

The signal significances are measured using the change in likelihood when the respective signal PDF component is removed from the likelihood function and the data is refit. Twice this change in likelihood is equivalent to a $\Delta \chi^{2}$ for the joint estimation of $p$ parameters, where $p$ is the number of parameters describing the signal PDF component that is removed from the total PDF. A one-sided Gaussian significance is calculated from the $\chi^{2}$ probability with $p$ degrees of freedom.

In the absence of a significant signal, an upper limit on the number of possible signal candidates is calculated by integrating the maximized-likelihood distribution for positive values of yield. When calculating these upper limits, the ratios of yields between the five $\Lambda_{c}^{+}$decay modes are Gaussian constrained based on estimated efficiency ratios, and world-average $\Lambda_{c}^{+}$branching ratios and their uncertainties [7]. Signal shape parameters are also Gaussian constrained with values and errors measured either from the charm-strange baryon isospin partners or other decay modes in which a signal is found in this analysis. These Gaussian constraints are multiplicative factors in the likelihood function,

$$
\mathcal{L}=\exp \left[-\sum_{k} \frac{\left(\mathcal{A}_{k}-a_{k}\right)^{2}}{2 \sigma_{k}^{2}}-\sum_{q} \frac{\left(\mathcal{R}_{q}-r_{q}\right)^{2}}{2 \sigma_{q}^{2}}\right] \exp \left[-\sum_{m=1}^{5}\left(N_{m}-\sum_{\eta} S_{m}^{\eta}-\sum_{\kappa} B_{m}^{\kappa}\right)\right] \times \prod_{m=1}^{5} L_{m}
$$

where $\mathcal{A}_{k}$ are the measured signal parameters with uncertainties $\sigma_{k}, \mathcal{R}_{q}$ are the yield ratios with uncertainties $\sigma_{q}$, and $a_{k}$ and $r_{q}$ are free parameters.

\subsubsection{Systematic Uncertainties}

Several sources of systematic error are investigated and quantified. The systematic errors on the measured masses, widths, yields, and fractions are summarized in Tables 4.14 and 4.15. Systematic 
errors on efficiencies are discussed at the end of this section. No significant errors are found due to the use of non-relativistic Breit-Wigner functions as opposed to relativistic Breit-Wigner functions. Also, no significant errors on the masses are found by comparing the masses of reconstructed candidates in the signal-MC samples to the generated masses. Systematic errors on efficiencies due to uncertainties on the $\Lambda^{0} \rightarrow p \pi^{-}$and $K_{s} \rightarrow \pi^{+} \pi^{-}$branching fractions are found to be insignificant relative to other sources of error. Systematic errors due to $\mathrm{MC}$ and data differences of $\Lambda_{c}^{+}, K_{s}$, and $\Lambda^{0}$ resolution are also found to be insignificant to other sources of error on efficiencies.

Changes to the fixed resolution parameters for $M_{\Xi_{c}}$ and $M_{\Sigma_{c}}$ are made to determine their effect on the measured masses, widths, yields, and resonant fractions. All Gaussian resolutions are increased by $10 \%$ in additional fits to the data. This increase of $10 \%$ is based on another BABAR analysis with an estimate of the resolution agreement between $\mathrm{MC}$ and data for the decay of $\Lambda_{c}^{+}$to $p K^{-} \pi^{+}$. The magnitude of the changes to the masses, widths, yields, and resonant fractions are used as symmetric systematic errors as listed in Tables 4.14 and 4.15. The $\Sigma_{c}(2455)^{++}$resolutions and fractions listed in both Table 4.8 and Table 4.7 vary because of the different values of $M_{\Xi_{c}}$ relative to the kinematic threshold. The averages of the two tables are used as resolution and fraction parameters in the fits to data. To quantify a systematic error for this process, the data are refit using the values from Table 4.8 and also refit using the values from Table 4.7. Again, the magnitude of the largest changes to the masses, widths, yields, and resonant fractions are used as symmetric systematic errors. Both sources of resolution error (for decays with a $\Sigma_{c}(2455)^{++}$) are added in quadrature and listed in Table 4.14. The $\Sigma_{c}(2455)^{0}$ resolutions and fractions listed in both Table 4.8 and Table 4.7 do not significantly vary and differ by less than $10 \%$.

The threshold functional shape in the PDF components (Equation 4.8) is evaluated for systematic errors by allowing additional shape parameters in the fit. The parameter $\beta$ is replaced by three separate parameters where one is used for the $T\left(M_{\Xi_{c}}\right)$ functions and two are used for the $T\left(M_{\Sigma_{c}}\right)$ functions. An additional threshold function in the $M_{\Sigma_{c}}$ variable is created for the non-resonant background component of the PDF:

$$
T\left(M_{\Sigma_{c}}\right) \rightarrow\left[f T_{a}\left(M_{\Sigma_{c}}\right)+(1-f) T_{b}\left(M_{\Sigma_{c}}\right)\right]
$$

where $f$ is the fraction of the new PDF component with the shape $T_{a}\left(M_{\Sigma_{c}}\right)$. The two $\beta$ parameters in $T_{a}\left(M_{\Sigma_{c}}\right)$ and $T_{b}\left(M_{\Sigma_{c}}\right)$ are separate free parameters. The data are refit with the modified PDFs and the magnitude of the changes in masses, widths, yields, and resonant fractions are used as symmetric systematic errors. The errors quantified with this procedure are listed in Tables 4.14 and 4.15 .

The phase-space functions (Equations 4.10 and 4.11) are not convolved with resolution functions. A systematic error due to this PDF inaccuracy is quantified by shifting the $M_{\Xi_{c}}$ and $M_{\Sigma_{c}}$ variables in the phase-space functions by one resolution width toward the phase-space thresholds. This is done separately for each of the two variables. The changes in masses, widths, yields, and resonant 
Table 4.14: Systematic uncertainties on $\Xi_{c}(2970)^{+}, \Xi_{c}(3055)^{+}, \Xi_{c}(3077)^{+}$, and $\Xi_{c}(3123)^{+}$threebody decay masses, widths, yields, and fractions. Uncertainties due to resolution effects, PDF shapes, phase-space approximations, the inclusion of the $\Xi_{c}(3123)^{+}$signal shape, and detector simulation are listed. The systematic errors from each source are added in quadrature. Res. refers to the fraction of signal that proceeds through an intermediate-resonant state. $\Sigma_{c}(2455)^{++}$refers to the fraction of the intermediate-resonant decays that are $\Sigma_{c}(2455)^{++} K^{-}$.

\begin{tabular}{lccccc}
\hline & $\begin{array}{c}\text { Mass } \\
\left(\mathrm{MeV} / c^{2}\right)\end{array}$ & $\begin{array}{c}\text { Width } \\
(\mathrm{MeV})\end{array}$ & $\begin{array}{c}\text { Yield } \\
\text { Res. }\end{array}$ & $\begin{array}{c}\Sigma_{c}(2455)^{++} \\
(\%)\end{array}$ \\
\hline \hline$\Xi_{c}(2970)^{+}$ & & & & & \\
Mass Resolution & \pm 0.6 & \pm 1.3 & \pm 27 & \pm 4.2 & - \\
Threshold PDF Shape & \pm 1.0 & \pm 0.3 & \pm 61 & \pm 8.1 & - \\
Phase-Space Function & \pm 1.2 & \pm 0.3 & \pm 76 & \pm 8.7 & - \\
Additional Signal PDF & \pm 0.4 & \pm 0.4 & \pm 26 & \pm 3.3 & - \\
Detector Simulation & \pm 0.1 & - & - & - & - \\
\hline Total & \pm 1.7 & \pm 1.5 & \pm 104 & \pm 13.0 & - \\
\hline$\Xi_{c}(3055)^{+}$ & & & & & \\
Mass Resolution & \pm 0.4 & \pm 7.3 & \pm 57 & - & - \\
Threshold PDF Shape & \pm 0.3 & \pm 6.8 & \pm 47 & - & - \\
Phase-Space Function & \pm 0.1 & \pm 4.2 & \pm 25 & - & - \\
Additional Signal PDF & \pm 0.0 & \pm 1.4 & \pm 10 & - & - \\
Detector Simulation & \pm 0.1 & - & - & - & - \\
\hline Total & \pm 0.5 & \pm 7.3 & \pm 79 & - & - \\
\hline$\Xi_{c}(3077)^{+}$ & & & & & \\
Mass Resolution & \pm 0.11 & \pm 0.4 & \pm 13 & \pm 3.6 & \pm 2.5 \\
Threshold PDF Shape & \pm 0.10 & \pm 0.2 & \pm 4 & \pm 1.2 & \pm 2.7 \\
Phase-Space Function & \pm 0.09 & \pm 0.3 & \pm 12 & \pm 1.4 & \pm 1.9 \\
Additional Signal PDF & \pm 0.05 & \pm 0.2 & \pm 20 & \pm 4.6 & \pm 2.4 \\
Detector Simulation & \pm 0.14 & - & - & - & - \\
\hline Total & \pm 0.18 & \pm 0.6 & \pm 27 & \pm 6.1 & \pm 4.8 \\
\hline$\Xi_{c}(3123)^{+}$ & & & & & \\
Mass Resolution & \pm 0.3 & \pm 1.5 & \pm 5 & - & - \\
Threshold PDF Shape & \pm 0.2 & \pm 0.6 & \pm 7 & - & - \\
Phase-Space Function & \pm 0.1 & \pm 0.5 & \pm 3 & - & - \\
Detector Simulation & \pm 0.1 & - & - & - & - \\
\hline Total & \pm 0.3 & \pm 1.7 & \pm 9 & - & - \\
\hline \hline
\end{tabular}


Table 4.15: Systematic uncertainties on $\Xi_{c}(2970)^{0}$ and $\Xi_{c}(3077)^{0}$ three-body decay masses, widths, yields, and fractions. Uncertainties due to resolution effects, PDF shapes, and phase-space approximations, and detector simulation are listed. The systematic errors from each source are added in quadrature. Res. refers to the fraction of signal that proceeds through an intermediate-resonant state. $\Sigma_{c}(2455)^{0}$ refers to the fraction of the intermediate-resonant decays that are $\Sigma_{c}(2455)^{0} K_{s}$.

\begin{tabular}{lccccc}
\hline & $\begin{array}{c}\text { Mass } \\
\left(\mathrm{MeV} / c^{2}\right)\end{array}$ & $\begin{array}{c}\text { Width } \\
(\mathrm{MeV})\end{array}$ & Yield & $\begin{array}{c}\text { Res. } \\
(\%)\end{array}$ & $\begin{array}{c}\Sigma_{c}(2455)^{0} \\
(\%)\end{array}$ \\
\hline \hline$\Xi_{c}(2970)^{0}$ & & & & & \\
Mass Resolution & \pm 0.6 & \pm 7.1 & \pm 11 & \pm 1 & - \\
Threshold PDF Shape & \pm 1.3 & \pm 3.7 & \pm 25 & \pm 14 & - \\
Phase-Space Function & \pm 0.7 & \pm 1.7 & \pm 8 & \pm 17 & - \\
Detector Simulation & \pm 0.1 & - & - & - & - \\
\hline Total & \pm 1.6 & \pm 8.2 & \pm 28 & \pm 22 & - \\
\hline$\Xi_{c}(3077)^{0}$ & & & & & \\
Mass Resolution & \pm 0.01 & \pm 0.3 & \pm 1 & \pm 0.4 & \pm 0.3 \\
Threshold PDF Shape & \pm 0.12 & \pm 0.2 & \pm 1 & \pm 4.1 & \pm 3.1 \\
Phase-Space Function & \pm 0.03 & \pm 0.1 & \pm 1 & \pm 2.4 & \pm 0.2 \\
Detector Simulation & \pm 0.14 & - & - & - & - \\
$\Xi_{c}(2970)^{0}$ & \pm 0.02 & \pm 1.5 & \pm 15 & \pm 0.9 & \pm 6.3 \\
\hline Total & \pm 0.19 & \pm 1.5 & \pm 15 & \pm 4.9 & \pm 7.0 \\
\hline
\end{tabular}


fractions for each of these shifts are added in quadrature and used as symmetric systematic errors.

Measurements of invariant mass with the BABAR Detector have systematic errors associated with SVT alignment, detector angular dependencies, energy-loss corrections, the solenoidal magnetic field, and material magnetization. These systematic errors were extensively studied for the BABAR Collaboration's precision measurement of the $\Lambda_{c}^{+}$mass [37] and were determined to contribute $\pm 0.14 \mathrm{MeV} / c^{2}$ total systematic error to the $\Lambda_{c}^{+}$mass measurement. The decay mode utilized in the $\Lambda_{c}^{+}$mass measurement $\left(\Lambda K_{S}^{0} K^{+}\right)$and the decay modes used in this analysis $\left(\Lambda_{c}^{+} K^{-} \pi^{+}\right.$and $\Lambda_{c}^{+} K_{s} \pi^{-}$) have similar $Q$-values, where the $Q$-value for a decay $A \rightarrow B+C+\ldots$ is defined as $Q=M(A)-M(B)-M(C)-\ldots$. These similar $Q$-values, along with our more stringent requirement on candidate momentum, lead us to believe that $\pm 0.14 \mathrm{MeV} / c^{2}$ is a conservative estimate for the systematic error from detector effects in this analysis. Reconstructed mass biases are also measured in studies of the signal-MC samples. The magnitudes of these mass biases are used as additional symmetric systematic errors on evaluated signal masses.

The signal PDF shape for $\Xi_{c}(2970)^{0}$ is included in the final fit to the data. Because the statistical significance of the $\Xi_{c}(2970)^{0}$ is small, systematic errors for the measurements of $\Xi_{c}(3077)^{0}$ are calculated based on their change when the $\Xi_{c}(2970)^{0}$ PDF component is not used. The changes are included as symmetric systematic errors. The statistical significance of the $\Xi_{c}(3123)^{+}$is also marginal. Systematic errors for the measurements of $\Xi_{c}(2970)^{+}, \Xi_{c}(3055)^{+}$, and $\Xi_{c}(3077)^{+}$are calculated from the changes when the $\Xi_{c}(3123)^{+}$PDF component is not used in the fit. These changes are used as symmetric systematic errors.

Signal reconstruction efficiencies have systematic errors due to differences in particle identification efficiency and tracking efficiency between signal-MC samples and data. Each signal-MC candidate has a weight applied to it to correct for these differences, and there are associated systematic errors as well. Systematic errors on efficiencies due to the particle identification are as follows: $1.0 \%$ per kaon, $1.0 \%$ per pion, and $4.0 \%$ per proton. A tracking efficiency systematic error of about $0.4 \%$ per track (added linearly) is also applied. There is a tracking efficiency correction and systematic error applied to all efficiencies calculated for decays channels with a $K_{s}$ meson or a $\Lambda^{0}$ baryon because they are reconstructed with vertices displaced from the $e^{+} e^{-}$interaction region. Tracks from $K_{s}$ meson or $\Lambda^{0}$ baryons are treated differently and have different systematic errors than tracks originating from the $e^{+} e^{-}$interaction region because these long lived neutral particles different interactions with detector material that they travel through. Correction factors range from about 0.98 to 1.05 per $K_{s}$ or $\Lambda^{0}$ candidate. The errors for these correction factors range from about \pm 0.01 to \pm 0.08 .

\subsubsection{Results for $\Lambda_{c}^{+} K^{-} \pi^{+}$}

The $\Lambda_{c}^{+} K^{-} \pi^{+}$data are fit with a PDF that includes components for $\Xi_{c}(2970)^{+}, \Xi_{c}(3055)^{+}, \Xi_{c}(3077)^{+}$, and $\Xi_{c}(3123)^{+}$signals. The measured masses, natural widths, and yields are listed in Table 4.16. Table 4.17 lists the $\Xi_{c}(2970)^{+}, \Xi_{c}(3055)^{+}, \Xi_{c}(3077)^{+}$, and $\Xi_{c}(3123)^{+}$yields by $\Lambda_{c}^{+}$decay mode with 
statistical and systematic errors. Table 4.18 lists the ratio of signal yields to the signal yields from the $\Lambda_{c}^{+} \rightarrow p K^{-} \pi^{+}$decay mode. The comparisons of these ratios reveal no statistically significant discrepancies. The fit to the data is illustrated in Figure 4.14 as $M_{\Xi_{c}}$ projections and in Figure 4.15 as $M_{\Sigma_{c}}$ projections. The two-dimensional normalized residual $(P-D) / \sqrt{D}$, where $P$ is the average $\mathrm{PDF}$ value in a bin and $D$ is the number of data candidates in a bin, is shown in Figure 4.16. A $\chi^{2}$ probability of $88 \%$ is calculated from this pull distribution. $M_{\Xi_{c}}$ projections for three $M_{\Sigma_{c}}$ ranges, using all five $\Lambda_{c}^{+}$decay modes, are shown in Figure $4.17 ; M_{\Sigma_{c}}$ ranges are illustrated in Figure 4.18.

In order to determine the statistical significance of each of the four signals, fits to the data are performed without each of the signal components, in turn.

- The maximum log-likelihood for the fit decreases by 80.8 units when the $\Xi_{c}(2970)^{+}$signal PDF is excluded from the fit. This decrease in maximum log-likelihood is doubled to calculate a $\chi^{2}$ probability with the joint estimation of eight parameters. The calculated probability that the background fluctuated up to the signal level $\left(<10^{-19}\right)$ corresponds to a one sided Gaussian significance greater than $9 \sigma$ for the $\Xi_{c}(2970)^{+}$signal.

- The maximum log-likelihood for the fit decreases by 31.0 units when the $\Xi_{c}(3055)^{+}$signal PDF is excluded from the fit. This decrease in maximum log-likelihood is doubled to calculate a $\chi^{2}$ probability with the joint estimation of seven parameters. The calculated probability that the background fluctuated up to the signal level $\left(6 \times 10^{-11}\right)$ corresponds to a significance of $6.4 \sigma$ for the $\Xi_{c}(3055)^{+}$signal.

- The maximum log-likelihood for the fit decreases by 90.7 units when the $\Xi_{c}(3077)^{+}$signal PDF is excluded from the fit. This decrease in maximum log-likelihood is doubled to calculate a $\chi^{2}$ probability with the joint estimation of nine parameters. The calculated probability that the background fluctuated up to the signal level $\left(<10^{-19}\right)$ corresponds to a significance greater than $9 \sigma$ for the $\Xi_{c}(3077)^{+}$signal.

- The maximum log-likelihood for the fit decreases by 14.5 units when the $\Xi_{c}(3123)^{+}$signal PDF is excluded from the fit. This decrease in maximum log-likelihood is doubled to calculate a $\chi^{2}$ probability with the joint estimation of seven parameters. The calculated probability that the background fluctuated up to the signal level $\left(1.4 \times 10^{-4}\right)$ corresponds to a significance of $3.6 \sigma$ for the $\Xi_{c}(3123)^{+}$signal.

The fractions of resonant and non-resonant decays are measured for the $\Xi_{c}(2970)^{+}$and the $\Xi_{c}(3077)^{+} ;(45 \pm 7 \pm 13) \%$ of the $\Xi_{c}(2970)^{+}$candidates are found to decay non-resonantly to $\Lambda_{c}^{+} K^{-} \pi^{+}$, while the rest of the signal candidates decay resonantly through a $\Sigma_{c}(2455)^{++} K^{-}$state. At $90 \%$ confidence level, at least $80 \%$ of the $\Xi_{c}(3077)^{+}$candidates are found to decay resonantly, while the rest of the signal candidates decay non-resonantly. Of the resonant $\Xi_{c}(3077)^{+}$decays, $(45 \pm 5 \pm 5) \%$ of the candidates decay resonantly through a $\Sigma_{c}(2455)^{++} K^{-}$state. The remainder 
Table 4.16: Masses, natural widths, yields, and significances of signals for $\Xi_{c}(2970)^{+}, \Xi_{c}(3055)^{+}$, $\Xi_{c}(3077)^{+}$, and $\Xi_{c}(3123)^{+}$decays to $\Lambda_{c}^{+} K^{-} \pi^{+}$.

\begin{tabular}{lcrrrc}
\hline & Mass $\left(\mathrm{MeV} / c^{2}\right)$ & \multicolumn{1}{c}{ Width $(\mathrm{MeV})$} & Yield & Significance \\
\hline \hline$\Xi_{c}(2970)^{+}$ & $2969.3 \pm 2.2 \pm 1.7$ & $26.7 \pm 7.6 \pm 1.5$ & $756 \pm 178 \pm 104$ & $>9 \sigma$ \\
$\Xi_{c}(3055)^{+}$ & $3054.2 \pm 1.2 \pm 0.5$ & $17.2 \pm 6.0 \pm 11.4$ & $218 \pm 53 \pm 79$ & $6.4 \sigma$ \\
$\Xi_{c}(3077)^{+}$ & $3077.0 \pm 0.4 \pm 0.2$ & $5.5 \pm 1.3 \pm 0.6$ & $403 \pm 54 \pm 27$ & $>9 \sigma$ \\
$\Xi_{c}(3123)^{+}$ & $3122.9 \pm 1.3 \pm 0.3$ & $4.4 \pm 3.4 \pm 1.7$ & $101 \pm 34 \pm$ & $3.6 \sigma$ \\
\hline \hline
\end{tabular}

of the candidates decay through a $\Sigma_{c}(2520)^{++} K^{-}$state. The $\Xi_{c}(3055)^{+}$and $\Xi_{c}(3123)^{+}$are found to only decay resonantly; the $\Xi_{c}(3055)^{+}$decays through a $\Sigma_{c}(2455)^{++} K^{-}$state and the $\Xi_{c}(3123)^{+}$ decays through a $\Sigma_{c}(2520)^{++} K^{-}$state. Other signal PDF components are not included for the $\Xi_{c}(3055)^{+}$and $\Xi_{c}(3123)^{+}$baryons in the final fit.

The production cross-section times branching fractions for $\Xi_{c}(2970)^{+}, \Xi_{c}(3055)^{+}, \Xi_{c}(3077)^{+}$, and $\Xi_{c}(3123)^{+}$decaying to $\Lambda_{c}^{+} K^{-} \pi^{+}$are calculated for each of the five $\Lambda_{c}^{+}$decay modes. These five separate values are listed in Table 4.19. A best linear unbiassed estimate (BLUE) technique [38] is used to combine the results

$$
\frac{N_{i}}{\epsilon_{i} \mathcal{R}_{i}}
$$

from the separate $\Lambda_{c}^{+}$decay modes $(i)$, where $N_{i}$ are the fitted yields, $\epsilon_{i}$ are the estimated efficiencies, and $\mathcal{R}_{i}$ are the ratios of each $\Lambda_{c}^{+}$branching fraction to $\mathcal{B}\left(\Lambda_{c}^{+} \rightarrow p K^{-} \pi^{+}\right)$[7]. All systematic errors on efficiencies are assumed to be $100 \%$ correlated. Muiltiplying the combined results by the integrated luminousity gives

$$
\begin{aligned}
& \sigma\left(\Xi_{c}(2970)^{+} X\right) \times \mathcal{B}\left(\Xi_{c}(2970)^{+} \rightarrow \Lambda_{c}^{+} K^{-} \pi^{+}\right) \times \mathcal{B}\left(\Lambda_{c}^{+} \rightarrow p K^{-} \pi^{+}\right)=(11.8 \pm 3.4 \pm 2.2) \mathrm{fb}, \\
& \sigma\left(\Xi_{c}(3055)^{+} X\right) \times \mathcal{B}\left(\Xi_{c}(3055)^{+} \rightarrow \Lambda_{c}^{+} K^{-} \pi^{+}\right) \times \mathcal{B}\left(\Lambda_{c}^{+} \rightarrow p K^{-} \pi^{+}\right)=(2.2 \pm 1.2 \pm 0.7) \mathrm{fb}, \\
& \sigma\left(\Xi_{c}(3077)^{+} X\right) \times \mathcal{B}\left(\Xi_{c}(3077)^{+} \rightarrow \Lambda_{c}^{+} K^{-} \pi^{+}\right) \times \mathcal{B}\left(\Lambda_{c}^{+} \rightarrow p K^{-} \pi^{+}\right)=(8.1 \pm 1.2 \pm 0.8) \mathrm{fb}, \quad \text { and } \\
& \sigma\left(\Xi_{c}(3123)^{+} X\right) \times \mathcal{B}\left(\Xi_{c}(3123)^{+} \rightarrow \Lambda_{c}^{+} K^{-} \pi^{+}\right) \times \mathcal{B}\left(\Lambda_{c}^{+} \rightarrow p K^{-} \pi^{+}\right)=(1.6 \pm 0.6 \pm 0.2) \mathrm{fb} .
\end{aligned}
$$

Three additional data sets are studied in more detail in order to determine if any of the peaking structures (charm-strange baryon signals) in the data are due to a misreconstructed signal of another source; this type of misreconstructed signal is known as a "reflection". These additional data sets are "wrong-sign" $\Lambda_{c}^{+} K^{+} \pi^{-}$data, the generic-ce MC sample, and data with the invariant mass $M\left(\Lambda_{c}^{+} K^{-} \pi^{+}\right)$recalculated as $M\left(\Lambda_{c}^{+} \pi^{-} \pi^{+}\right)$using the pion mass as opposed to the kaon mass. These three data sets include all five of the $\Lambda_{c}^{+}$decay modes. 

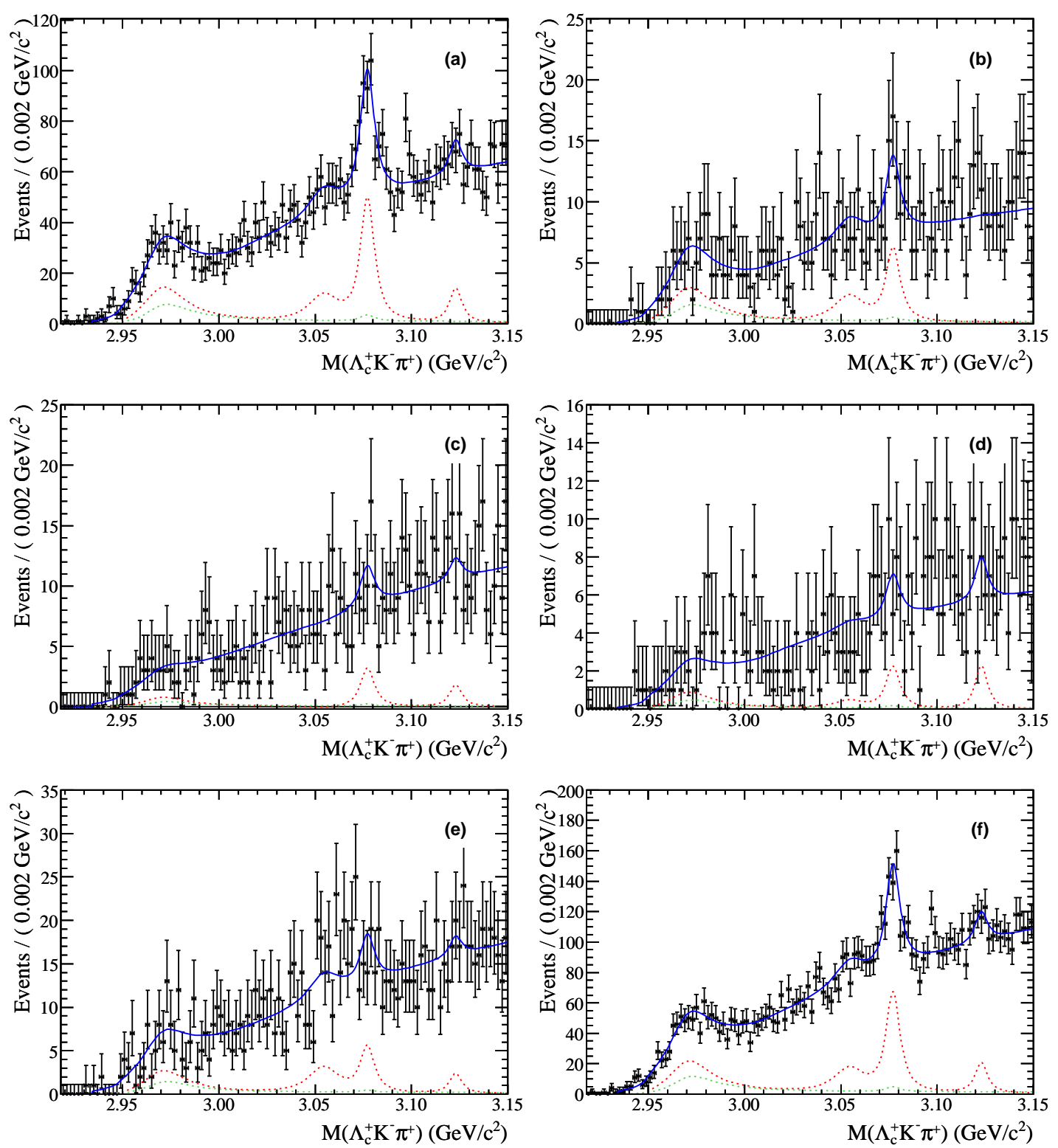

Figure 4.14: $M\left(\Lambda_{c}^{+} K^{-} \pi^{+}\right)$projections of $\Lambda_{c}^{+} K^{-} \pi^{+}$candidates in data (points with error bars) and their fit (blue curves). The dotted red curves represent the resonant signal components and the dotted green curves represent the non-resonant signal components. The $\Lambda_{c}^{+}$candidates are reconstructed from (a) $p K^{-} \pi^{+}$, (b) $p K_{s}$, (c) $p K_{s} \pi^{-} \pi^{+}$, (d) $\Lambda^{0} \pi^{+}$, (e) $\Lambda^{0} \pi^{+} \pi^{-} \pi^{+}$, and (f) all five final states. 

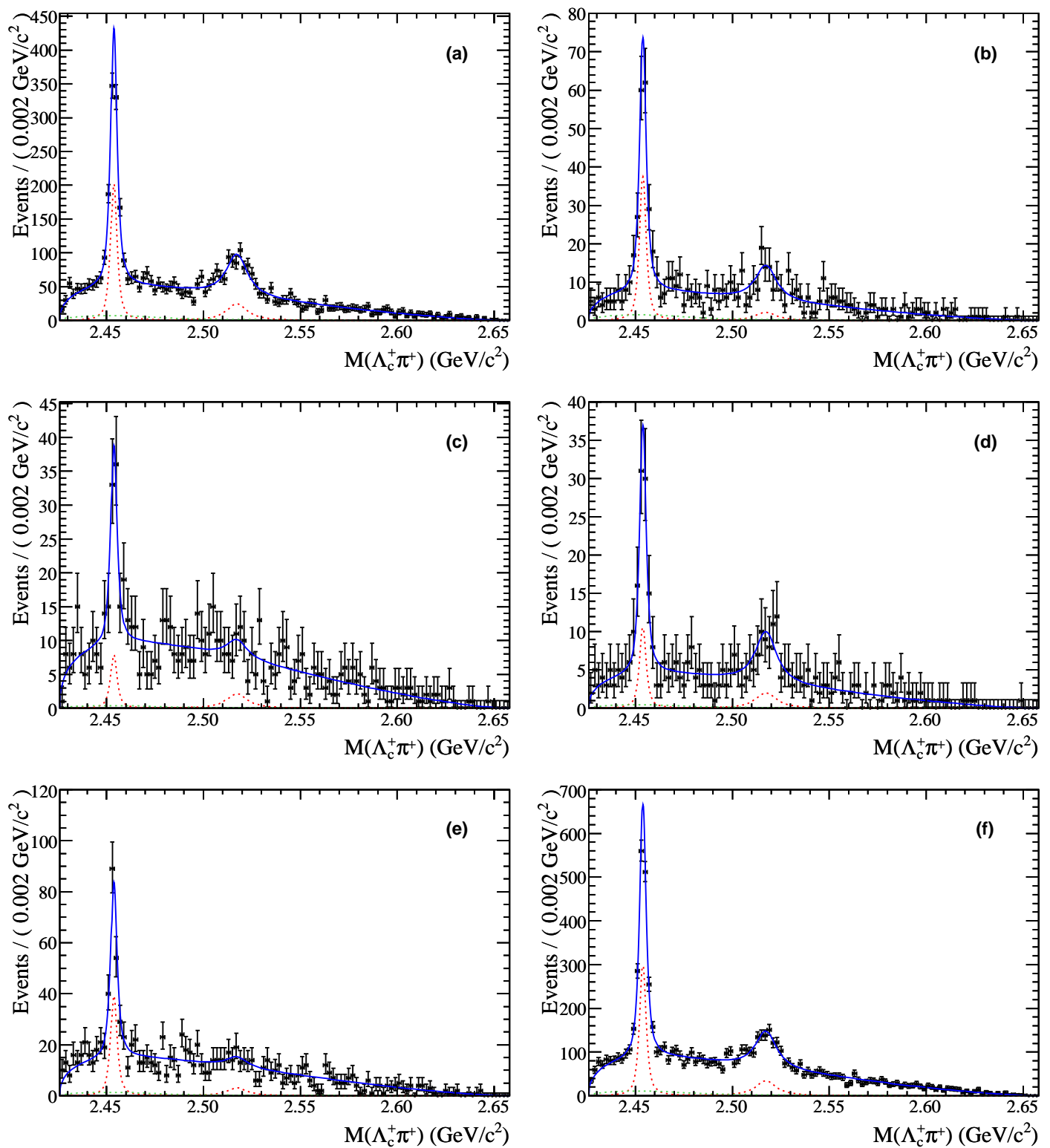

Figure 4.15: $M\left(\Lambda_{c}^{+} \pi^{+}\right)$projections of $\Lambda_{c}^{+} K^{-} \pi^{+}$candidates in data (points with error bars) and their fit (blue curves). The dotted red curves represent the resonant signal components and the dotted green curves represent the non-resonant signal components. The $\Lambda_{c}^{+}$candidates are reconstructed from (a) $p K^{-} \pi^{+}$, (b) $p K_{s}$, (c) $p K_{s} \pi^{-} \pi^{+}$, (d) $\Lambda^{0} \pi^{+}$, (e) $\Lambda^{0} \pi^{+} \pi^{-} \pi^{+}$, and (f) all five final states. 
Table 4.17: Yields of $\Xi_{c}(2970)^{+}, \Xi_{c}(3055)^{+}, \Xi_{c}(3077)^{+}$, and $\Xi_{c}(3123)^{+}$signals by $\Lambda_{c}^{+}$decay mode. Errors are statistical and systematic, respectively.

\begin{tabular}{lcrrrr}
\hline & $\Xi_{c}(2970)^{+}$ & $\Xi_{c}(3055)^{+}$ & $\Xi_{c}(3077)^{+}$ & $\Xi_{c}(3123)^{+}$ \\
\hline \hline$p K^{-} \pi^{+}$ & $501 \pm 123 \pm 87$ & $144 \pm 39 \pm 47$ & $301 \pm 42 \pm 19$ & $68 \pm 23 \pm 4$ \\
$p K_{s}$ & $103 \pm 27 \pm 8$ & $29 \pm 13 \pm 17$ & $37 \pm 12 \pm 6$ & $0 \pm 6 \pm 4$ \\
$p K_{s} \pi^{+} \pi^{-}$ & $27 \pm 14 \pm 10$ & $-2 \pm 6 \pm 4$ & $20 \pm 9 \pm 2$ & $9 \pm 8 \pm 2$ \\
$\Lambda^{0} \pi^{+}$ & $31 \pm 14 \pm 5$ & $7 \pm 6 \pm 2$ & $14 \pm 8 \pm 2$ & $12 \pm 8 \pm 3$ \\
$\Lambda^{0} \pi^{+} \pi^{-} \pi^{+}$ & $94 \pm 28 \pm 11$ & $41 \pm 14 \pm 16$ & $32 \pm 12 \pm 3$ & $11 \pm 9 \pm 3$ \\
\hline \hline
\end{tabular}

Table 4.18: Signal yield ratios relative to the $\Lambda_{c}^{+} \rightarrow p K \pi$ modes. Errors are statistical only.

\begin{tabular}{lcccc}
\hline & $\Xi_{c}(2970)^{+}$ & $\Xi_{c}(3055)^{+}$ & $\Xi_{c}(3077)^{+}$ & $\Xi_{c}(3123)^{+}$ \\
\hline \hline$p K_{s}$ & $0.21 \pm 0.07$ & $0.20 \pm 0.10$ & $0.12 \pm 0.04$ & $0.00 \pm 0.09$ \\
$p K_{s} \pi^{+} \pi^{-}$ & $0.05 \pm 0.03$ & $-0.01 \pm 0.04$ & $0.07 \pm 0.03$ & $0.13 \pm 0.12$ \\
$\Lambda^{0} \pi^{+}$ & $0.06 \pm 0.03$ & $0.04 \pm 0.05$ & $0.05 \pm 0.03$ & $0.17 \pm 0.13$ \\
$\Lambda^{0} \pi^{+} \pi^{-} \pi^{+}$ & $0.19 \pm 0.07$ & $0.28 \pm 0.12$ & $0.11 \pm 0.04$ & $0.17 \pm 0.14$ \\
\hline \hline
\end{tabular}

Table 4.19: Production cross-sections times branching fractions for charm-strange baryons decaying to $\Lambda_{c}^{+} K_{s} \pi^{-}$and $\Lambda_{c}^{+} K^{-} \pi^{+}$for each of the five $\Lambda_{c}^{+}$decay modes. All quantities are in $\mathrm{fb}$.

\begin{tabular}{lcccccc}
\hline & $\Xi_{c}(2970)^{0}$ & $\Xi_{c}(3077)^{0}$ & $\Xi_{c}(2970)^{+}$ & $\Xi_{c}(3055)^{+}$ & $\Xi_{c}(3077)^{+}$ & $\Xi_{c}(3123)^{+}$ \\
\hline \hline$\times \mathcal{B}\left(p K^{-} \pi^{+}\right)$ & $9.7 \pm 6.4$ & $9.7 \pm 3.2$ & $15.0 \pm 4.5$ & $4.2 \pm 1.6$ & $9.0 \pm 1.8$ & $2.0 \pm 0.7$ \\
$\times \mathcal{B}\left(p K_{s}\right)$ & $2.1 \pm 5.7$ & $16.7 \pm 8.1$ & $23.8 \pm 7.4$ & $6.4 \pm 3.2$ & $8.7 \pm 3.0$ & $0.1 \pm 1.4$ \\
$\times \mathcal{B}\left(p K_{s} \pi^{+} \pi^{-}\right)$ & $18.1 \pm 16.3$ & $3.0 \pm 10.3$ & $13.7 \pm 7.9$ & $-1.0 \pm 3.0$ & $10.8 \pm 5.2$ & $4.9 \pm 4.5$ \\
$\times \mathcal{B}\left(\Lambda^{0} \pi^{+}\right)$ & $7.1 \pm 8.0$ & $-0.3 \pm 3.7$ & $10.3 \pm 5.0$ & $1.7 \pm 2.3$ & $4.2 \pm 2.7$ & $3.2 \pm 2.4$ \\
$\times \mathcal{B}\left(\Lambda^{0} \pi^{+} \pi^{-} \pi^{+}\right)$ & $-1.4 \pm 7.7$ & $19.7 \pm 8.9$ & $23.2 \pm 8.3$ & $9.3 \pm 3.6$ & $8.3 \pm 3.3$ & $2.9 \pm 2.3$ \\
\hline \hline
\end{tabular}




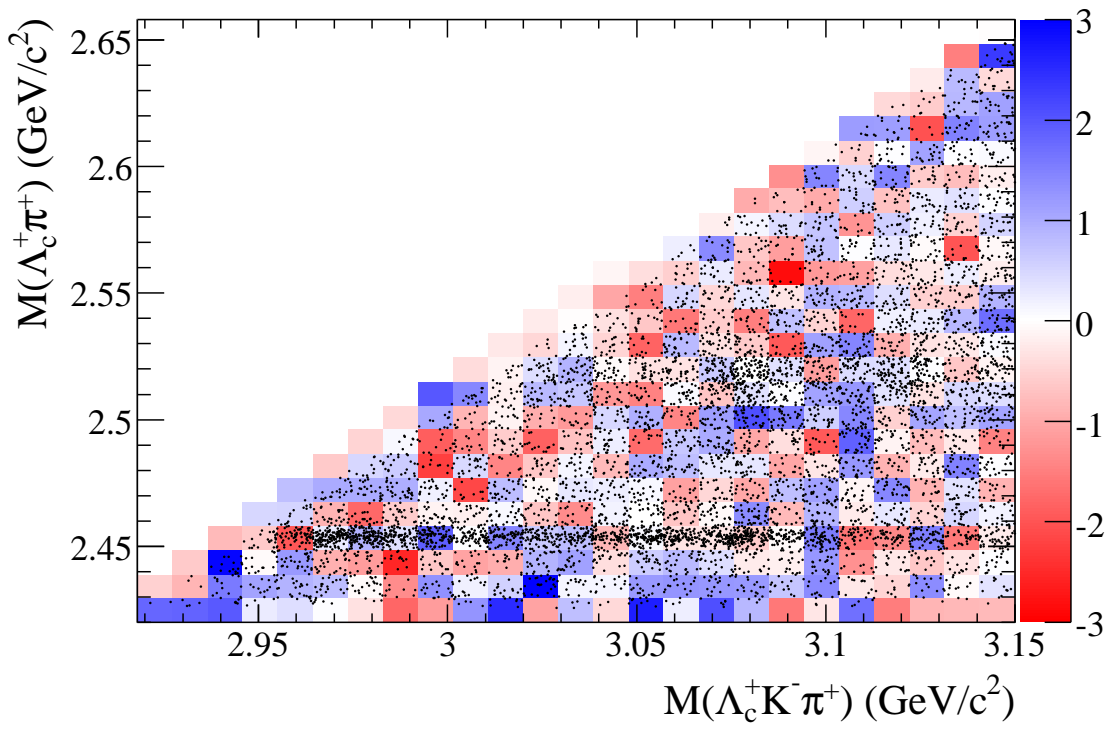

Figure 4.16: Two-dimensional normalized residuals (colored bins) for reconstructed $\Lambda_{c}^{+} K^{-} \pi^{+}$candidates in data (points) and their fit. A $\chi^{2}$ probability of $88 \%$ is calculated from this pull distribution using bins that have at least 11 candidates.

- A scatter plot of the wrong-sign data is shown in Figure 4.19 with three $M_{\Sigma_{c}}$ regions indicated by colored horizontal lines as in Figure 4.18. $M_{\Xi_{c}}$ projections of these three regions are shown in Figure 4.20. The projections in Figure 4.20 reveal no hidden peaking structure in the wrong-sign data. Also, the resonant and non-resonant background PDFs appear to describe the background shape very well.

- A scatter plot of the generic- $c \bar{c}$ MC sample is shown in Figure 4.21 with three $M_{\Sigma_{c}}$ regions indicated by colored horizontal lines. $M_{\Xi_{c}}$ projections of these three regions are shown in Figure 4.23. The projections in Figure 4.23 reveal no hidden peaking structure in the generic$c \bar{c}$ MC sample.

- A scatter plot of the data with $M_{\Xi_{c}}$ recalculated as $M\left(\Lambda_{c}^{+} \pi^{-} \pi^{+}\right)$is shown in Figure 4.22 with three $M_{\Sigma_{c}}$ regions indicated by colored horizontal lines. $M\left(\Lambda_{c}^{+} \pi^{-} \pi^{+}\right)$projections of these three regions are shown in Figure 4.24. The projections in Figure 4.24 reveal no hidden peaking structure in the recalculated $M\left(\Lambda_{c}^{+} \pi^{-} \pi^{+}\right)$data.

The lack of peaking structure in each of these three data sets is evidence that all four signals for excited $\Xi_{c}$ baryons are real and not reflections of other signals.

The possibility of observing reflections of excited $\Lambda_{c}^{+}$baryons is further studied by calculating the central values of where reflections would occur. In these calculations, the mass of the pion is replaced with the mass of a kaon in the $\pi^{-}$four-vector. For $\Lambda_{c}(2593)^{+}, \Lambda_{c}(2625)^{+}, \Lambda_{c}(2765)^{+}$, 

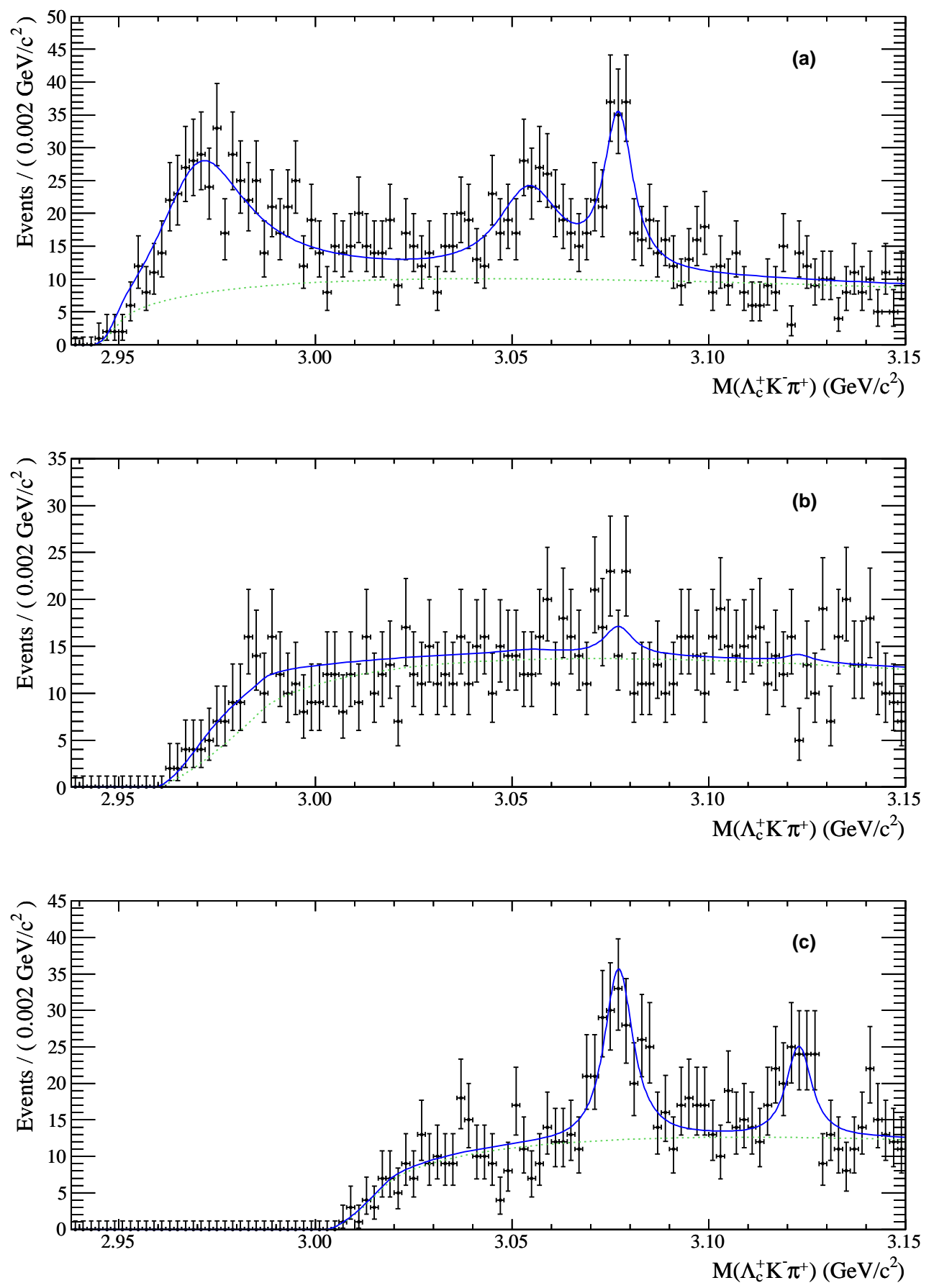

Figure 4.17: $M_{\Xi_{c}}$ projections of $\Lambda_{c}^{+} K^{-} \pi^{+}$candidates in data with a fit of $\Xi_{c}(2970)^{+}, \Xi_{c}(3055)^{+}$, $\Xi_{c}(3077)^{+}$, and $\Xi_{c}(3123)^{+}$. Plots (a), (b), and (c) are projection of different $M_{\Sigma_{c}}$ regions as indicated in Figure 4.18 


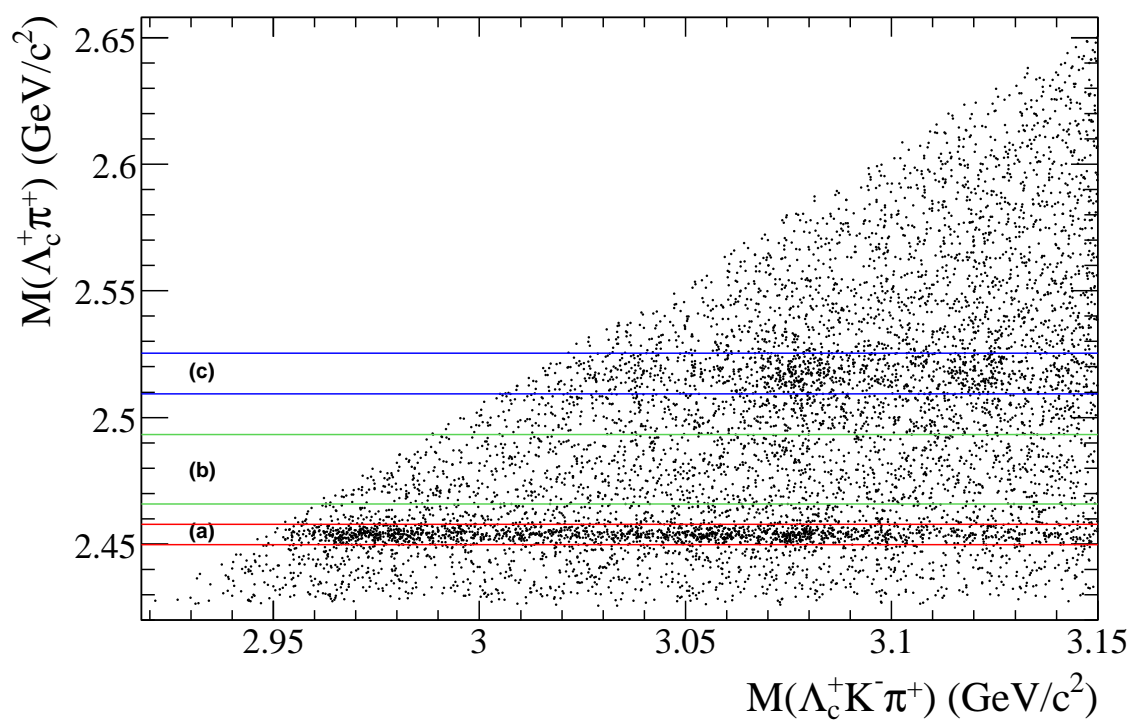

Figure 4.18: Scatter plot of $\Lambda_{c}^{+} K^{-} \pi^{+}$candidates in data. The horizontal lines indicate different regions of $M_{\Sigma_{c}}$ that are projected in Figure 4.17. The red lines indicate (a) the $\Sigma_{c}(2455)^{++}$region. The blue lines indicate (c) the $\Sigma_{c}(2520)^{++}$region. The green lines indicate (b) in between the $\Sigma_{c}(2455)^{++}$and $\Sigma_{c}(2520)^{++}$regions.

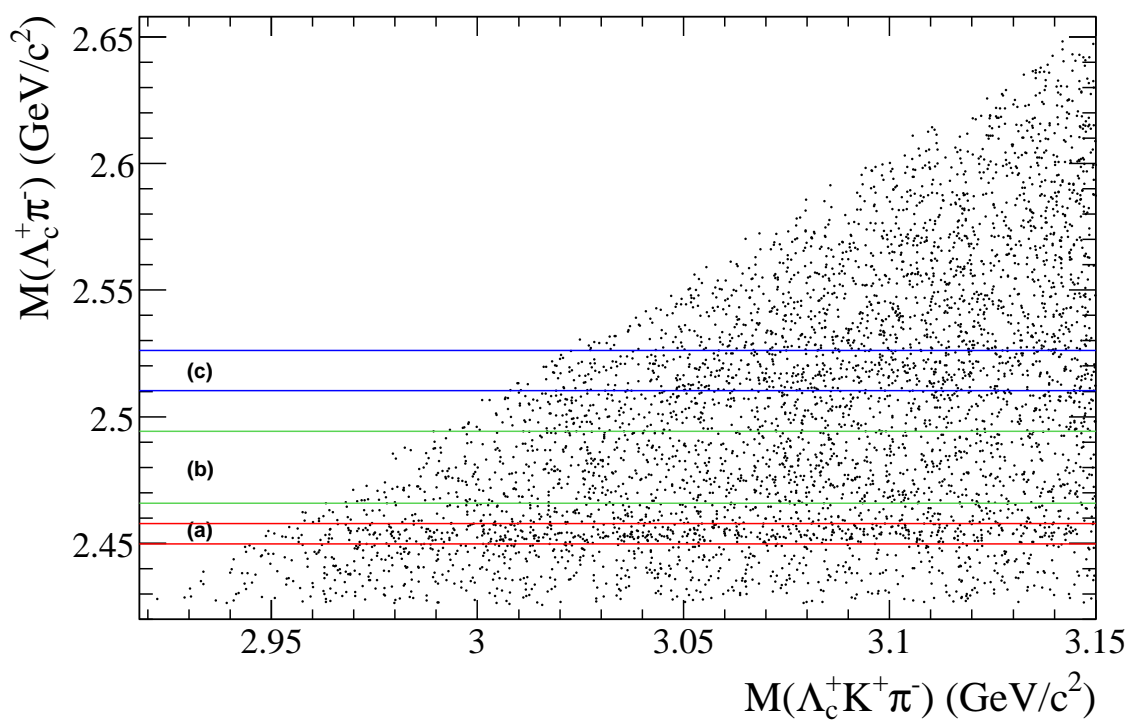

Figure 4.19: Scatter plot of wrong-sign $\Lambda_{c}^{+} K^{+} \pi^{-}$data. The horizontal lines indicate different regions of $M_{\Sigma_{c}}$ that are projected in Figure 4.20 . The red lines indicate (a) the $\Sigma_{c}(2455)^{0}$ region. The blue lines indicate (c) the $\Sigma_{c}(2520)^{0}$ region. The green lines indicate (b) in between the $\Sigma_{c}(2455)^{0}$ and $\Sigma_{c}(2520)^{0}$ regions. 

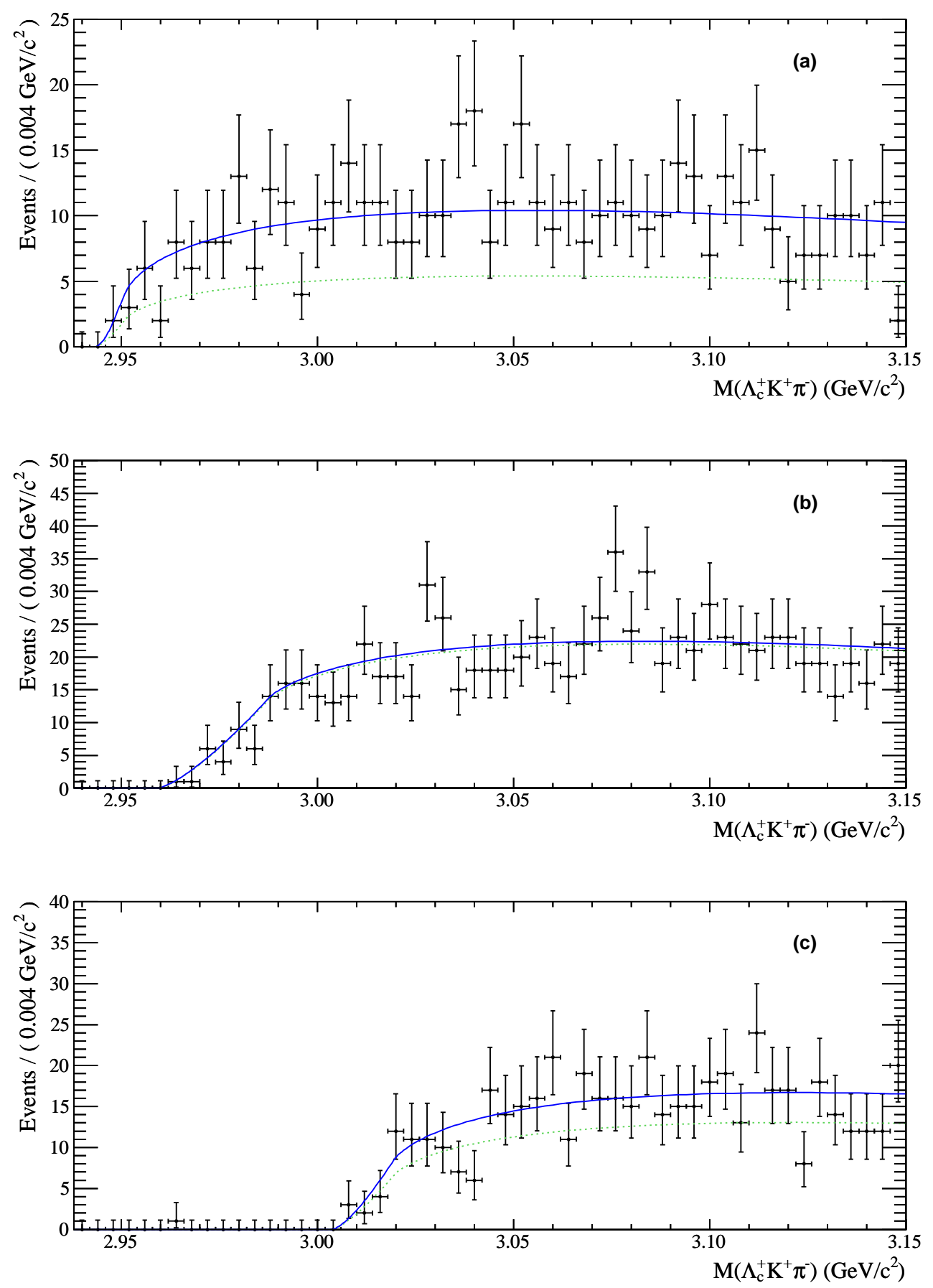

Figure 4.20: $M_{\Xi_{c}}$ projections of wrong-sign $\Lambda_{c}^{+} K^{+} \pi^{-}$data (points with error bars). The blue curves illustrate the total background PDF components and the dotted green curves illustrate the non-resonant background PDF component. Plots (a), (b), and (c) project out different $M_{\Sigma_{c}}$ regions as indicated in Figure 4.19. 


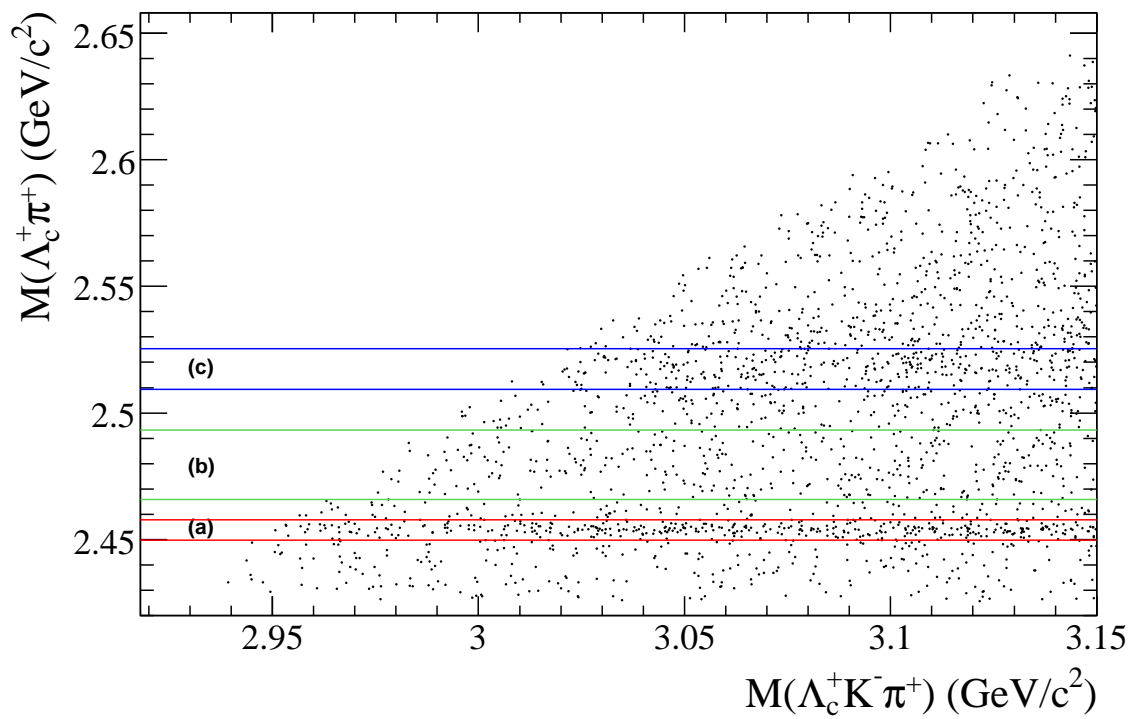

Figure 4.21: Scatter plot of the $\Lambda_{c}^{+} K^{-} \pi^{+}$reconstructed generic- $c \bar{c}$ MC sample. The horizontal lines indicate different regions of $M_{\Sigma_{c}}$ that are projected in Figure 4.23. The red lines indicate the region (a) which encompasses the $\Sigma_{c}(2455)^{++}$mass. The blue lines indicate the region (c) which encompasses the $\Sigma_{c}(2520)^{++}$mass. The green lines indicate the region (b) which is in between the $\Sigma_{c}(2455)^{++}$and $\Sigma_{c}(2520)^{++}$masses. 


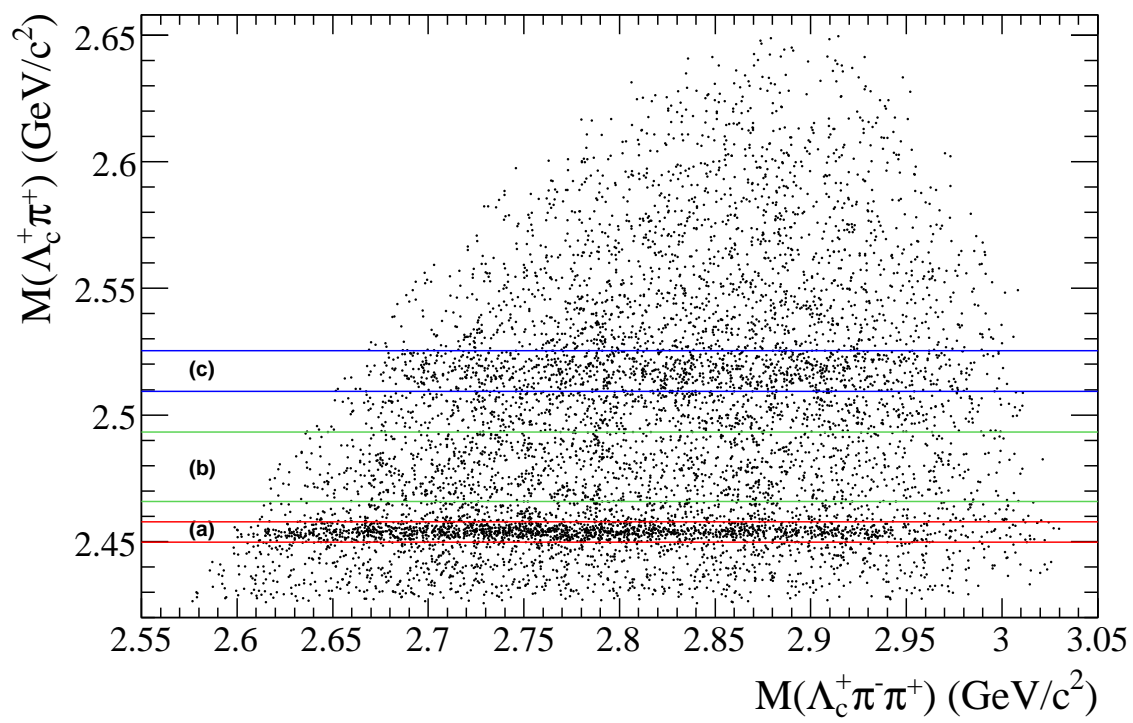

Figure 4.22: Scatter plot of the data with $M_{\Xi_{c}}$ recalculated as $M\left(\Lambda_{c}^{+} \pi^{-} \pi^{+}\right)$. The horizontal lines indicate different regions of $M_{\Sigma_{c}}$ that are projected in Figure 4.24. The red lines indicate the region (a) which encompasses the $\Sigma_{c}(2455)^{++}$mass. The blue lines indicate the region (c) which encompasses the $\Sigma_{c}(2520)^{++}$mass. The green lines indicate the region (b) which is in between the $\Sigma_{c}(2455)^{++}$and $\Sigma_{c}(2520)^{++}$masses. 

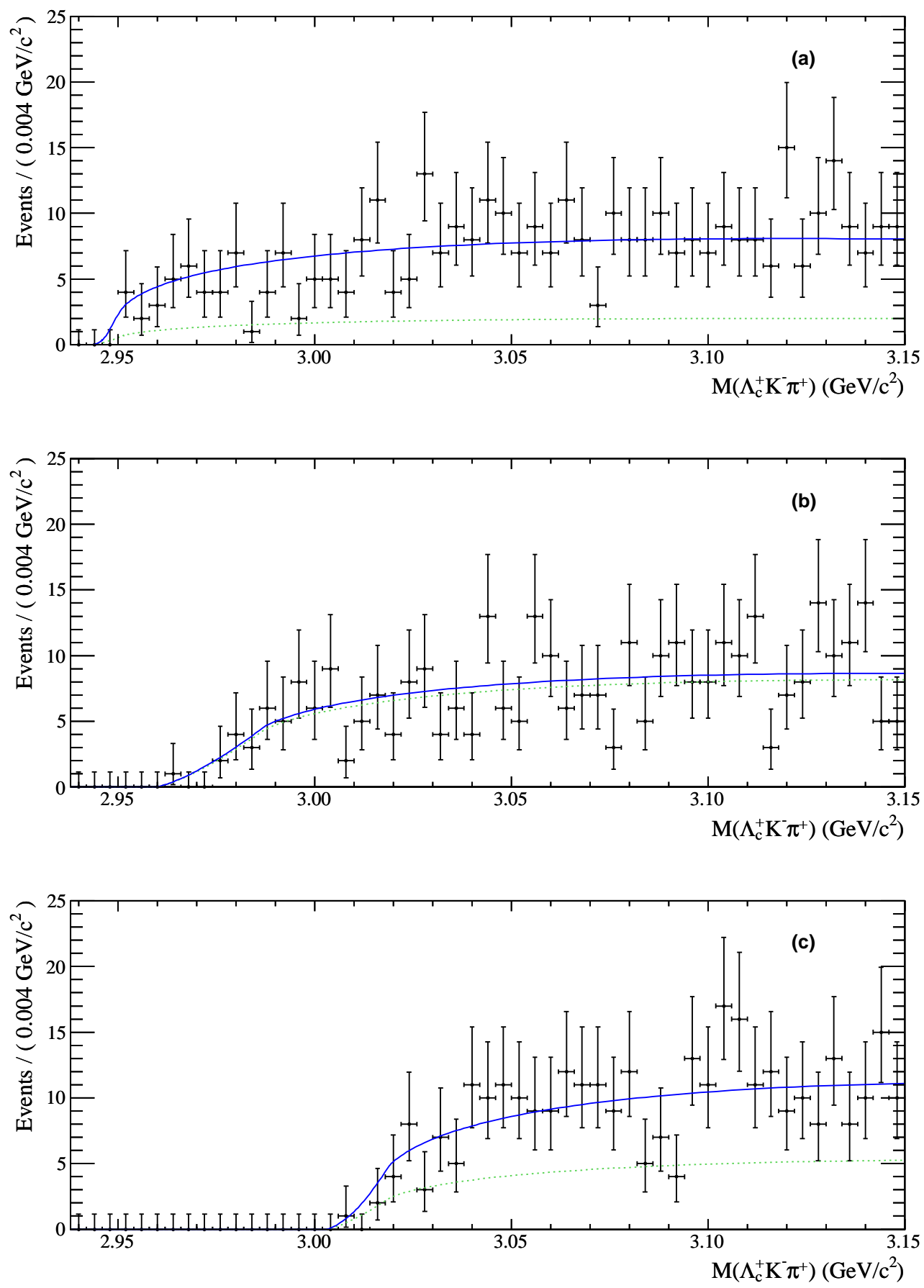

Figure 4.23: $M_{\Xi_{c}}$ projections of the $\Lambda_{c}^{+} K^{-} \pi^{+}$reconstructed generic- $c \bar{c} \mathrm{MC}$ sample. The blue curves illustrate the total background PDF components and the dotted green curves illustrate the nonresonant background PDF component. Plots (a), (b), and (c) project out different $M_{\Sigma_{c}}$ regions as indicated in Figure 4.21. 

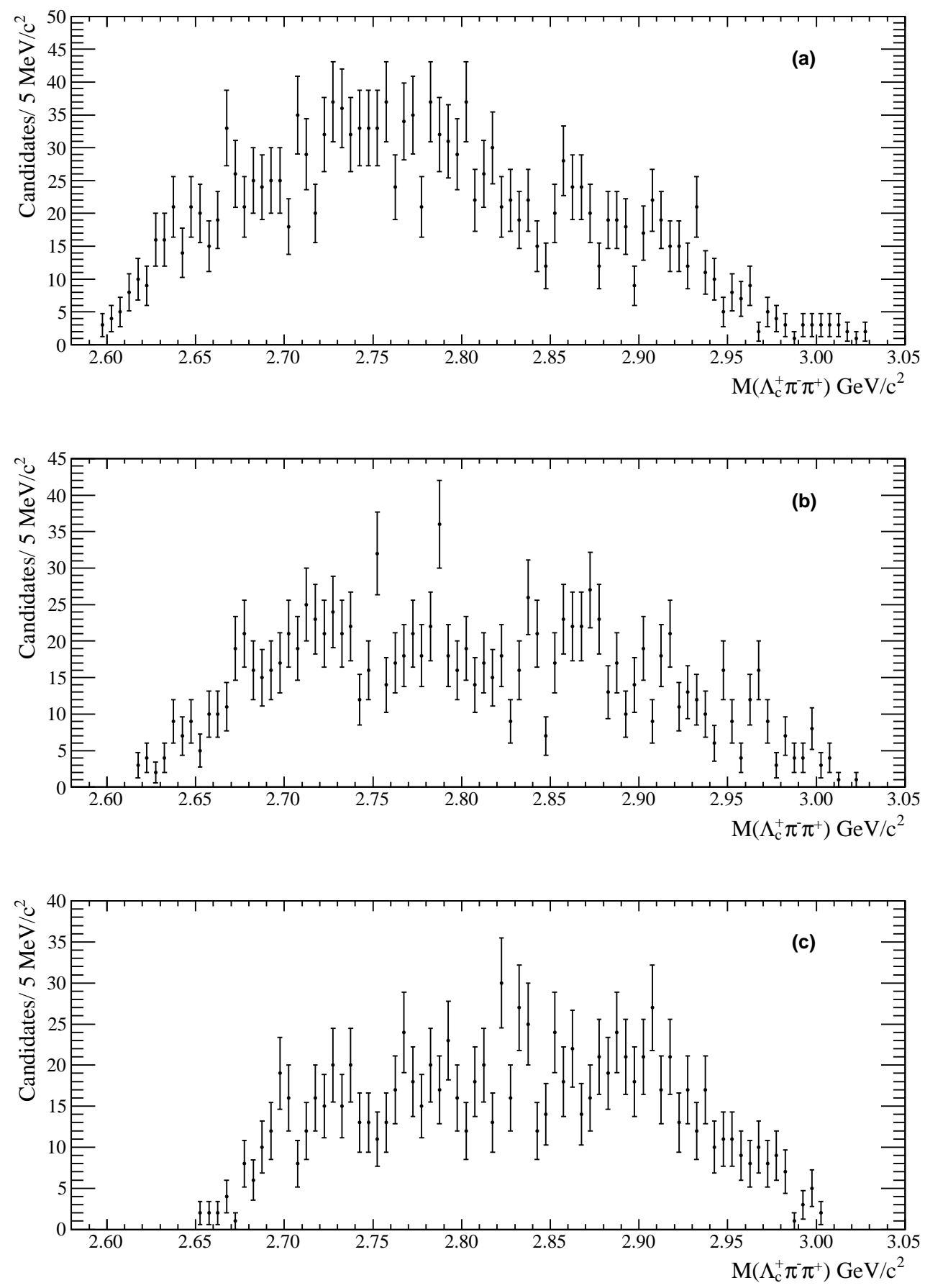

Figure 4.24: $M_{\Xi_{c}}$ projections of the data where $M_{\Xi_{c}}$ has been recalculated as $M\left(\Lambda_{c}^{+} \pi^{-} \pi^{+}\right)$. Plots (a), (b), and (c) project out different $M_{\Sigma_{c}}$ regions as indicated in Figure 4.22. 
and $\Lambda_{c}(2880)^{+}$baryons decaying resonantly through a $\Sigma_{c}(2455)^{++} \pi^{-}$state, the reflections would be centered at $2948 \mathrm{MeV} / c^{2}, 2960 \mathrm{MeV} / c^{2}, 3028 \mathrm{MeV} / c^{2}$, and $3102 \mathrm{MeV} / c^{2}$ in $M\left(\Lambda_{c}^{+} K^{-} \pi^{+}\right)$, respectively. For $\Lambda_{c}(2765)^{+}$and $\Lambda_{c}(2880)^{+}$baryons decaying resonantly through a $\Sigma_{c}(2520)^{++} \pi^{-}$state, the reflections would be centered at $3057 \mathrm{MeV} / c^{2}$, and $3124 \mathrm{MeV} / c^{2}$, respectively. The central values for these reflextions can be compared with the data in Figure 4.17. Of these six possible reflections, only $\Lambda_{c}(2880)^{+} \rightarrow \Sigma_{c}(2520)^{++} K^{-}$has a mass consistent with any of the observed excited $\Xi_{c}^{+}$baryons. However, the number of observable $\Lambda_{c}(2880)^{+}$baryons is considerably smaller than the three other excited $\Lambda_{c}^{+}$states and its decay branching fraction to $\Sigma_{c}(2520)^{++} \pi^{-}$is small. Furthermore, the measured natural width of the $\Xi_{c}(3123)^{+}$is as narrow as observed for the $\Lambda_{c}(2880)^{+}$. A reflection would have a wider shape than the real reconstructed signal. Analysis of $\Lambda_{c}(2880)^{+}$signal-MC samples reveals that a reflection of this signal would be several tens of $\mathrm{MeV} / \mathrm{c}^{2}$ wide and skewed towards higher masses.

Another test of the validity of the excited charm-strange baryon signals is that their $p^{*}$ should be distributed towards higher values than the combinatorial background. Two new data sets are produced from the previous $\Lambda_{c}^{+} K^{-} \pi^{+}$data set; one data set requires that the reconstructed $p^{*}$ of the signal candidate be greater than $3.5 \mathrm{GeV} / c$ while the other requires that the reconstructed $p^{*}$ of the signal candidate be greater than $4.0 \mathrm{GeV} / c$. Figure 4.25 shows the $M_{\Xi_{c}}$ projections of the data, in regions as defined in Figure 4.18, with $p^{*}>3.5$ and their fit. Figure 4.26 shows the $M_{\Xi_{c}}$ projections of the data, also in regions as defined in Figure 4.18, with $p^{*}>4.0$ and their fit. With $p^{*}>3.5$, the $\Xi_{c}(2970)^{+}, \Xi_{c}(3055)^{+}, \Xi_{c}(3077)^{+}$, and $\Xi_{c}(3123)^{+}$yields are $430 \pm 48,89 \pm 21,237 \pm 26$, and $35 \pm 13$, respectively. With $p^{*}>4.0$, the $\Xi_{c}(2970)^{+}, \Xi_{c}(3055)^{+}, \Xi_{c}(3077)^{+}$, and $\Xi_{c}(3123)^{+}$yields are $132 \pm 20,43 \pm 12,51 \pm 11,7 \pm 6$, respectively. All four peaking structures remain in these two data sets with higher $p^{*}$ requirements for the signal candidates. This supports the hypothesis that these peaking structures are not fluctuations in the combinatorial background.

\subsubsection{Results for $\Lambda_{c}^{+} K_{s} \pi^{-}$}

The $\Lambda_{c}^{+} K_{s} \pi^{-}$data are fit to determine $\Xi_{c}(2970)^{0}$ and $\Xi_{c}(3077)^{0}$ signal masses, natural widths, yields, and resonant fractions. Because of the low signal and background yields for this data, the mean and natural width parameters for $\Sigma_{c}(2455)^{0}$ and $\Sigma_{c}(2520)^{0}$ are fixed to PDG 2006 values. The fitted signal masses, natural widths, and resonant fractions are listed in Table 4.20 with statistical and systematic errors. The fitted signal yields are listed in Table 4.21 with statistical and systematic errors. Figure 4.27 shows the $M_{\Xi_{c}}$ projections of the data and their fit result while Figure 4.28 shows the $M_{\Sigma_{c}}$ projections. The two-dimensional pull $(P-D) / \sqrt{D}$, where $P$ is the average PDF value in a bin and $\mathrm{D}$ is the number of data candidates in a bin, is shown in Figure 4.29. A $\chi^{2}$ probability of $62 \%$ is calculated for this pull distribution using bins that have at least 11 candidates.

In order to determine the statistical significance of the $\Xi_{c}(2970)^{0}$ and $\Xi_{c}(3077)^{0}$ signals, fits to the data are performed without each of the signal components in turn. The maximum log-likelihood 

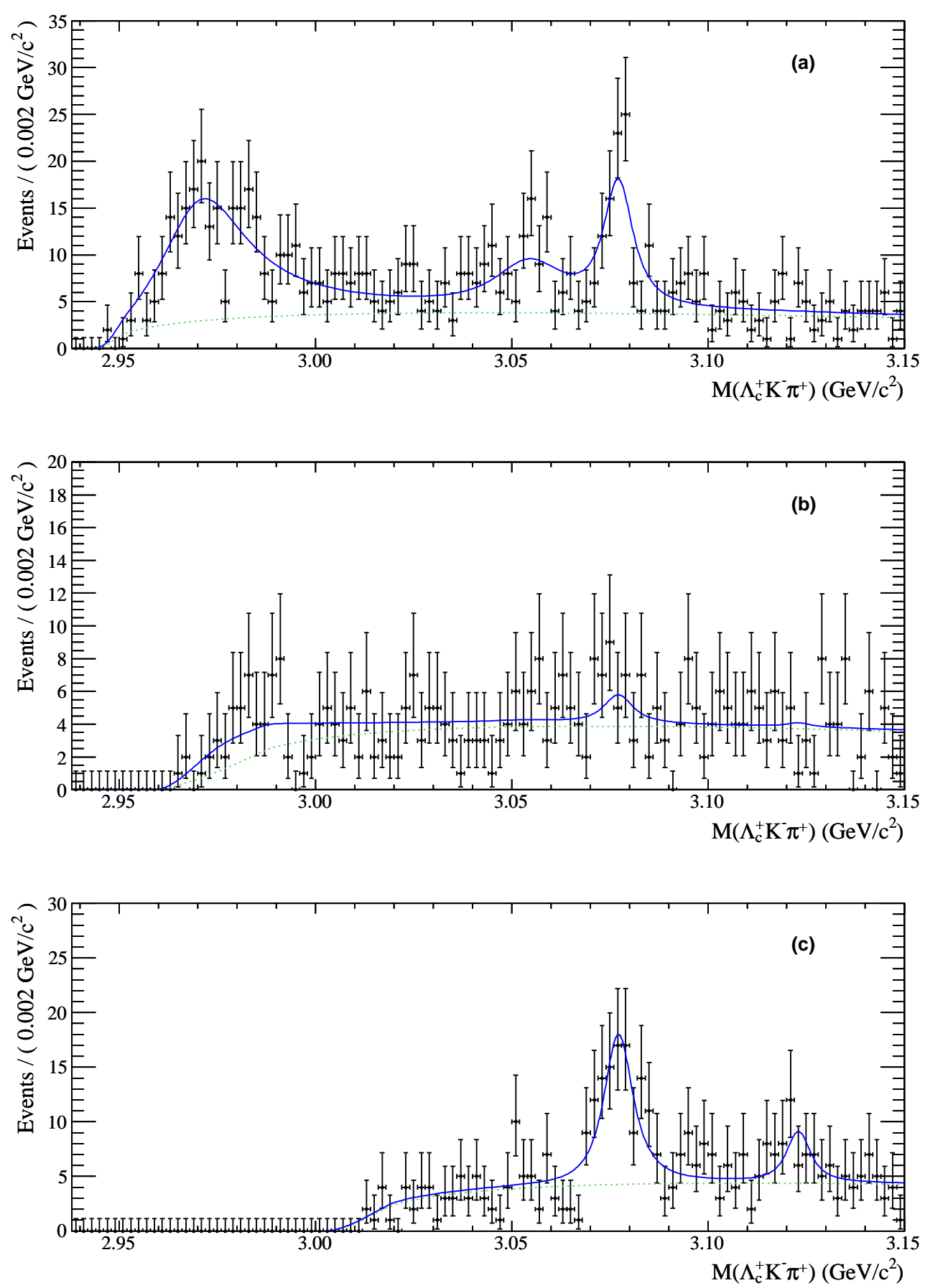

Figure 4.25: $M_{\Xi_{c}}$ projections of $\Lambda_{c}^{+} K^{-} \pi^{+}$candidates with $p^{*}>3.5$ (points with error bars) and their fit (blue lines). The dotted green lines illustrate the background PDF projections. Plots (a), (b), and (c) are project out different $M_{\Sigma_{c}}$ regions as indicated in Figure 4.18. 

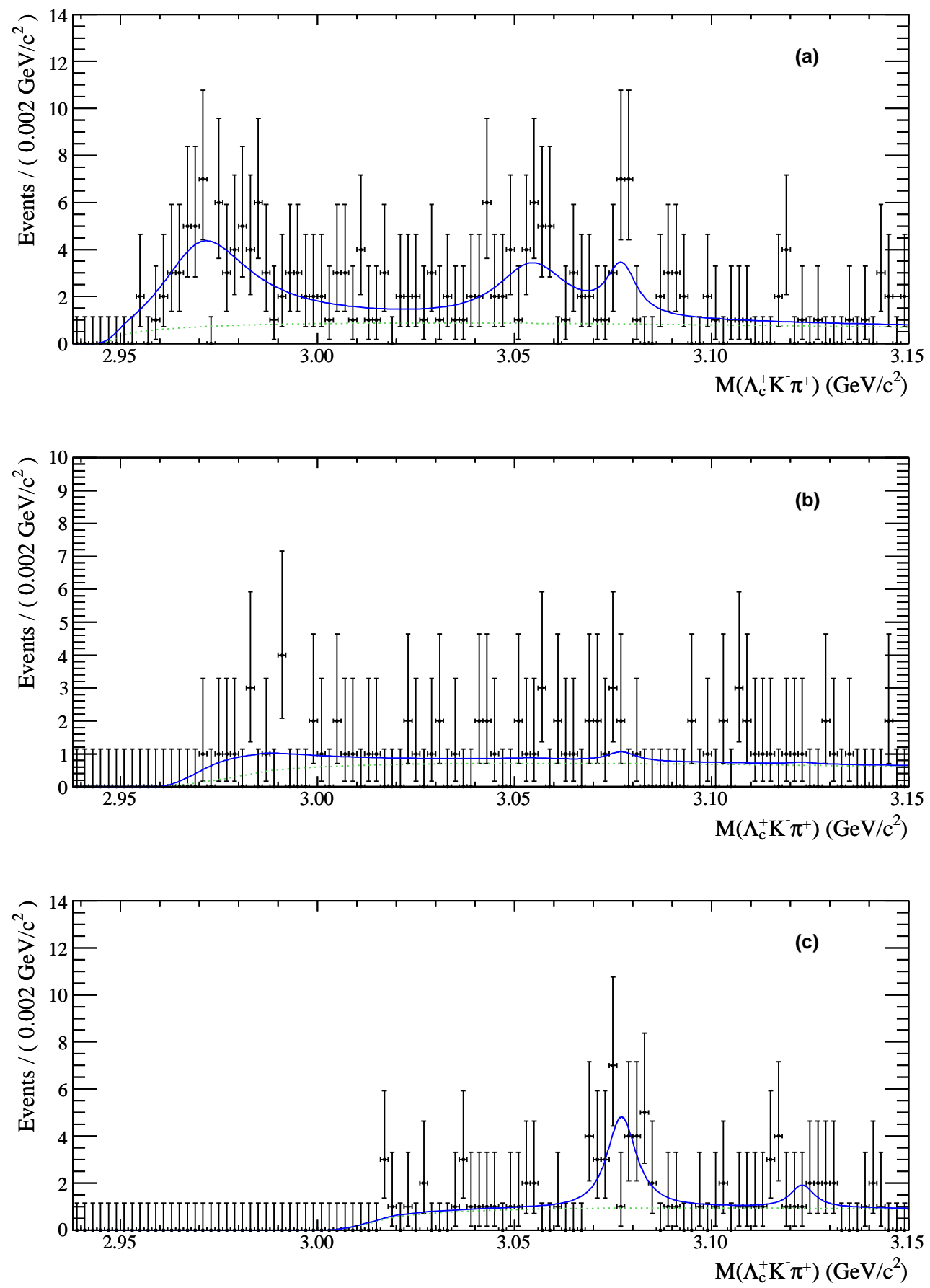

Figure 4.26: $M_{\Xi_{c}}$ projections of $\Lambda_{c}^{+} K^{-} \pi^{+}$candidates with $p^{*}>4.0$ (points with error bars) and their fit (blue lines). The dotted green lines illustrate the background PDF projections. Plots (a), (b), and (c) are project out different $M_{\Sigma_{c}}$ regions as indicated in Figure 4.18. 
for the fit decreases by 8.0 units when the $\Xi_{c}(2970)^{0}$ signal PDF is excluded from the fit. This decrease in maximum log-likelihood, with the joint estimation of eight parameters (mass, natural width, five yields, and resonant fraction), corresponds to a $1.7 \sigma$ significance for the $\Xi_{c}(2970)^{0}$ signal. The maximum log-likelihood for the fit decreases by 20.7 units when the $\Xi_{c}(3077)^{0}$ signal PDF is excluded from the fit. This decrease in maximum log-likelihood, with the joint estimation of nine parameters, corresponds to a $4.5 \sigma$ significance for the $\Xi_{c}(3077)^{0}$ signal. These significances are listed in Table 4.20. $(82 \pm 48 \pm 22) \%$ of the $\Xi_{c}(2970)^{0}$ is found to decay resonantly through $\Sigma_{c}(2455)^{0}$ while the rest decays non-resonantly. $(78 \pm 12 \pm 5) \%$ of the $\Xi_{c}(3077)^{0}$ is found to decay resonantly through $\Sigma_{c}(2455)^{0}$ or $\Sigma_{c}(2520)^{0}$ while the rest decays non-resonantly. $(44 \pm 12 \pm 7) \%$ of the $\Xi_{c}(3077)^{0}$ resonant decays are through $\Sigma_{c}(2455)^{0}$. Fits to the data that include PDF components for $\Xi_{c}(3055)^{0}$ and $\Xi_{c}(3123)^{0}$ increase the fit likelihood by 4.7 and 2.4 , respectively, which reveals no significant signals for these states.

The presence of a $\Xi_{c}(2970)^{0}$ state, being the isospin partner of $\Xi_{c}(2970)^{+}$, is expected. Despite the low statistical significance of the $\Xi_{c}(2970)^{0}$ signal, measurements for the $\Xi_{c}(3077)^{0}$ are performed with the inclusion of a $\Xi_{c}(2970)^{0}$ signal component in the PDF. Systematic errors on the $\Xi_{c}(3077)^{0}$ mass, natural width, yield, and resonant fractions are calculated based on changes resulting from the exclusion and inclusion of the $\Xi_{c}(2970)^{0}$ signal component. These systematic errors are listed in Table 4.15. Figure 4.30 shows the $M_{\Xi_{c}}$ projections of the data and their fit result including only the $\Xi_{c}(3077)^{0}$ signal PDF and Figure 4.31 shows the $M_{\Sigma_{c}}$ projections. The two-dimensional pull including only the $\Xi_{c}(3077)^{0}$ signal PDF is shown in Figure 4.32 . A $\chi^{2}$ probability of $54 \%$ is calculated for this pull distribution using bins that have at least 11 candidates.

The production cross-section times branching fractions for $\Xi_{c}(2970)^{0}$ decaying to $\Lambda_{c}^{+} K_{s} \pi^{-}$as well as for $\Xi_{c}(3077)^{0}$ decaying to $\Lambda_{c}^{+} K_{s} \pi^{-}$are calculated for each of the five $\Lambda_{c}^{+}$decay modes. These five separate values are listed in Table 4.19. A BLUE technique [38] is used to combine the results

$$
\frac{N_{i}}{\epsilon_{i} \mathcal{R}_{i}}
$$

from the separite $\Lambda_{c}^{+}$decay modes $(i)$, where $N_{i}$ are the fitted yields, $\epsilon_{i}$ are the estimated efficiencies, and $\mathcal{R}_{i}$ are the ratios of each $\Lambda_{c}^{+}$branching fraction to $\mathcal{B}\left(\Lambda_{c}^{+} \rightarrow p K^{-} \pi^{+}\right)$[7]. All systematic errors on efficiencies are considered $100 \%$ correlated. Muiltiplying the combined results by the integrated luminousity gives

$$
\begin{aligned}
& \sigma\left(\Xi_{c}(2970)^{0} X\right) \times \mathcal{B}\left(\Xi_{c}(2970)^{0} \rightarrow \Lambda_{c}^{+} \bar{K}^{0} \pi^{-}\right) \times \mathcal{B}\left(\Lambda_{c}^{+} \rightarrow p K^{-} \pi^{+}\right)=(4.1 \pm 3.3 \pm 2.8) \mathrm{fb}, \quad \text { and } \\
& \sigma\left(\Xi_{c}(3077)^{0} X\right) \times \mathcal{B}\left(\Xi_{c}(3077)^{0} \rightarrow \Lambda_{c}^{+} \bar{K}^{0} \pi^{-}\right) \times \mathcal{B}\left(\Lambda_{c}^{+} \rightarrow p K^{-} \pi^{+}\right)=(6.2 \pm 2.1 \pm 1.5) \mathrm{fb} .
\end{aligned}
$$


As no significant signal is found for $\Xi_{c}(2970)^{0}$, a $90 \%$ confidence-level (CL) upper limit is determined for its cross-section times branching fractions. The upper limit is calculated from integrating the likelihood distribution for fixed cross-sections times braching fractions above zero. When calculating the likelihood for the upper limit, Gaussian constraints are added for the ratio of fitted yields between the various $\Lambda_{c}^{+}$decay modes. These yield ratios between the five $\Lambda_{c}^{+}$decay modes are constrained according to ratios of estimated efficiencies and $\Lambda_{c}^{+}$branching ratios taken from the Particle Data Group [7], and their errors. Also, the mean, natural width, and resonant fraction of the signal is given Gaussian constraints to those measured from the $\Xi_{c}(2970)^{0}$ signal shape using both statistical and systematic errors. The measured 90\% CL upper limit on production cross-section times branching fractions is

$$
\sigma\left(\Xi_{c}(2970)^{0} X\right) \times \mathcal{B}\left(\Xi_{c}(2970)^{0} \rightarrow \Lambda_{c}^{+} \bar{K}^{0} \pi^{-}\right) \times \mathcal{B}\left(\Lambda_{c}^{+} \rightarrow p K^{-} \pi^{+}\right)<15.3 \mathrm{fb} .
$$

The corresponding upper limit on the yield of $\Xi_{c}(2970)^{0} \rightarrow \Lambda_{c}^{+} K_{s} \pi^{-}$is 87. Upper limits are also measured for the baryons $\Xi_{c}(3055)^{0}$ and $\Xi_{c}(3123)^{0}$; they are possible isospin partners of $\Xi_{c}(3055)^{+}$ and $\Xi_{c}(3123)^{+}$which are observed in $\Lambda_{c}^{+} K^{-} \pi^{+}$decays. These upper limits are calulated in the same way as just stated, but with the means and natural widths of the signals given Gaussian constraints to those measured from the $\Lambda_{c}^{+} K^{-} \pi^{+}$signal shapes. For $\Xi_{c}(3055)^{0}$, the measured $90 \%$ CL upper limit on production cross-section times branching fractions is

$$
\sigma\left(\Xi_{c}(3055)^{0} X\right) \times \mathcal{B}\left(\Xi_{c}(3055)^{0} \rightarrow \Lambda_{c}^{+} \bar{K}^{0} \pi^{-}\right) \times \mathcal{B}\left(\Lambda_{c}^{+} \rightarrow p K^{-} \pi^{+}\right)<7.3 \mathrm{fb} .
$$

The corresponding upper limit on the yield of $\Xi_{c}(3055)^{0} \rightarrow \Lambda_{c}^{+} K_{s} \pi^{-}$is 47 . For $\Xi_{c}(3123)^{0}$, the measured 90\% CL upper limit on production cross-section times branching fractions is

$$
\sigma\left(\Xi_{c}(3123)^{0} X\right) \times \mathcal{B}\left(\Xi_{c}(3123)^{0} \rightarrow \Lambda_{c}^{+} \bar{K}^{0} \pi^{-}\right) \times \mathcal{B}\left(\Lambda_{c}^{+} \rightarrow p K^{-} \pi^{+}\right)<1.4 \mathrm{fb} .
$$

The measured $95 \%$ CL upper limit on the yield of $\Xi_{c}(3123)^{0} \rightarrow \Lambda_{c}^{+} K_{s} \pi^{-}$is 9.6.

\subsection{Two-Body Decays}

The search for excited charm-strange baryons decaying to $\Lambda_{c}^{+} K_{s}$ and $\Lambda_{c}^{+} K^{-}$is described in this section. I search for signal in the range $2.91 \mathrm{GeV} / c^{2}$ to $3.15 \mathrm{GeV} / c^{2}$ in the $M\left(\Lambda_{c}^{+} K_{s}\right)$ and $M\left(\Lambda_{c}^{+} K^{-}\right)$ invariant-mass distributions. The data are divided into five subsamples based on the reconstructed 

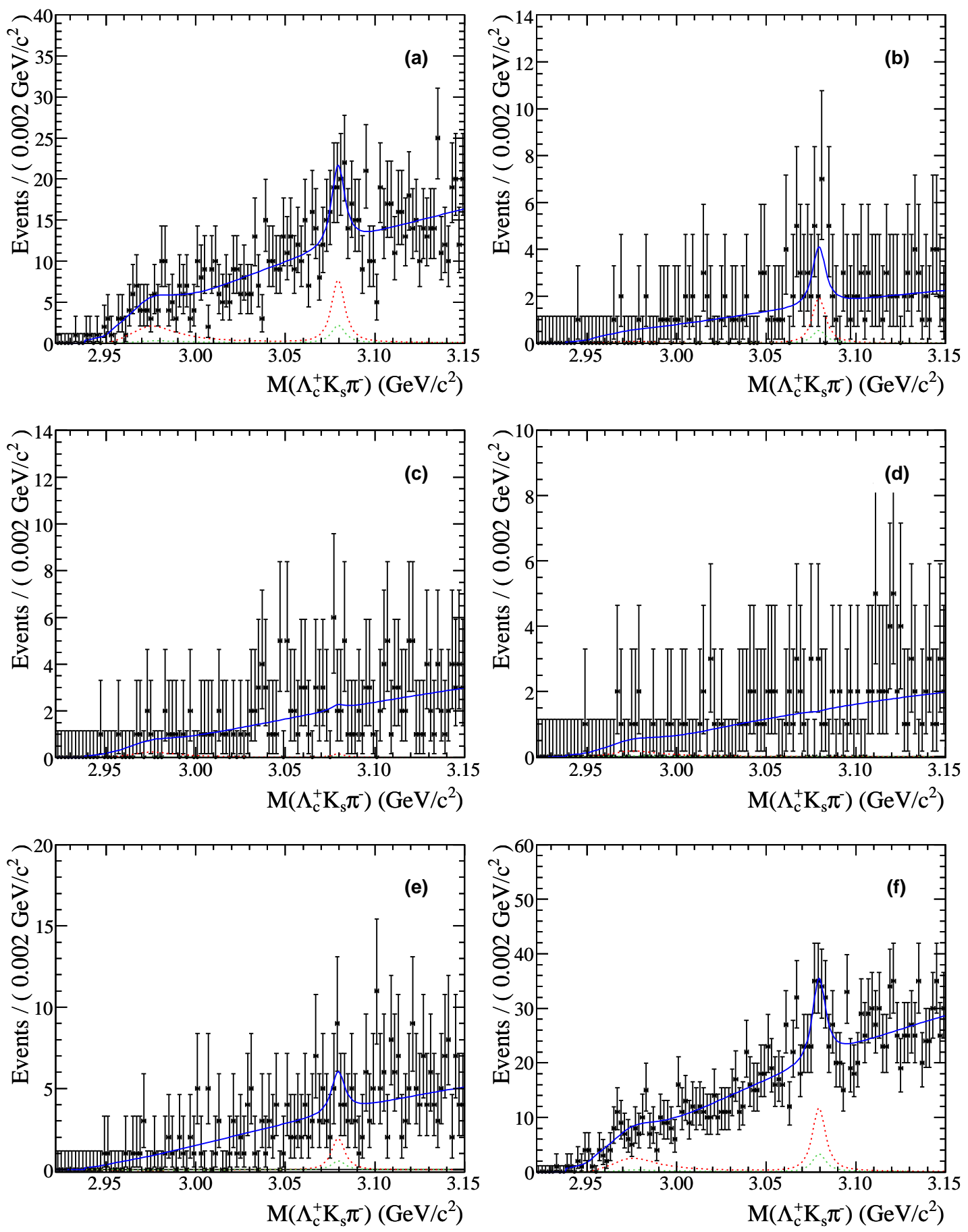

Figure 4.27: $M\left(\Lambda_{c}^{+} K_{s} \pi^{-}\right)$projections of $\Lambda_{c}^{+} K_{s} \pi^{-}$candidates in data (points with error bars) and theri fit (blue curves). The dotted red curves represent the resonant signal components and the dotted green curves represent the non-resonant signal components. The $\Lambda_{c}^{+}$candidates are reconstructed from (a) $p K^{-} \pi^{+}$, (b) $p K_{s}$, (c) $p K_{s} \pi^{-} \pi^{+}$, (d) $\Lambda^{0} \pi^{+}$, (e) $\Lambda^{0} \pi^{+} \pi^{-} \pi^{+}$, and (f) all five final states. 

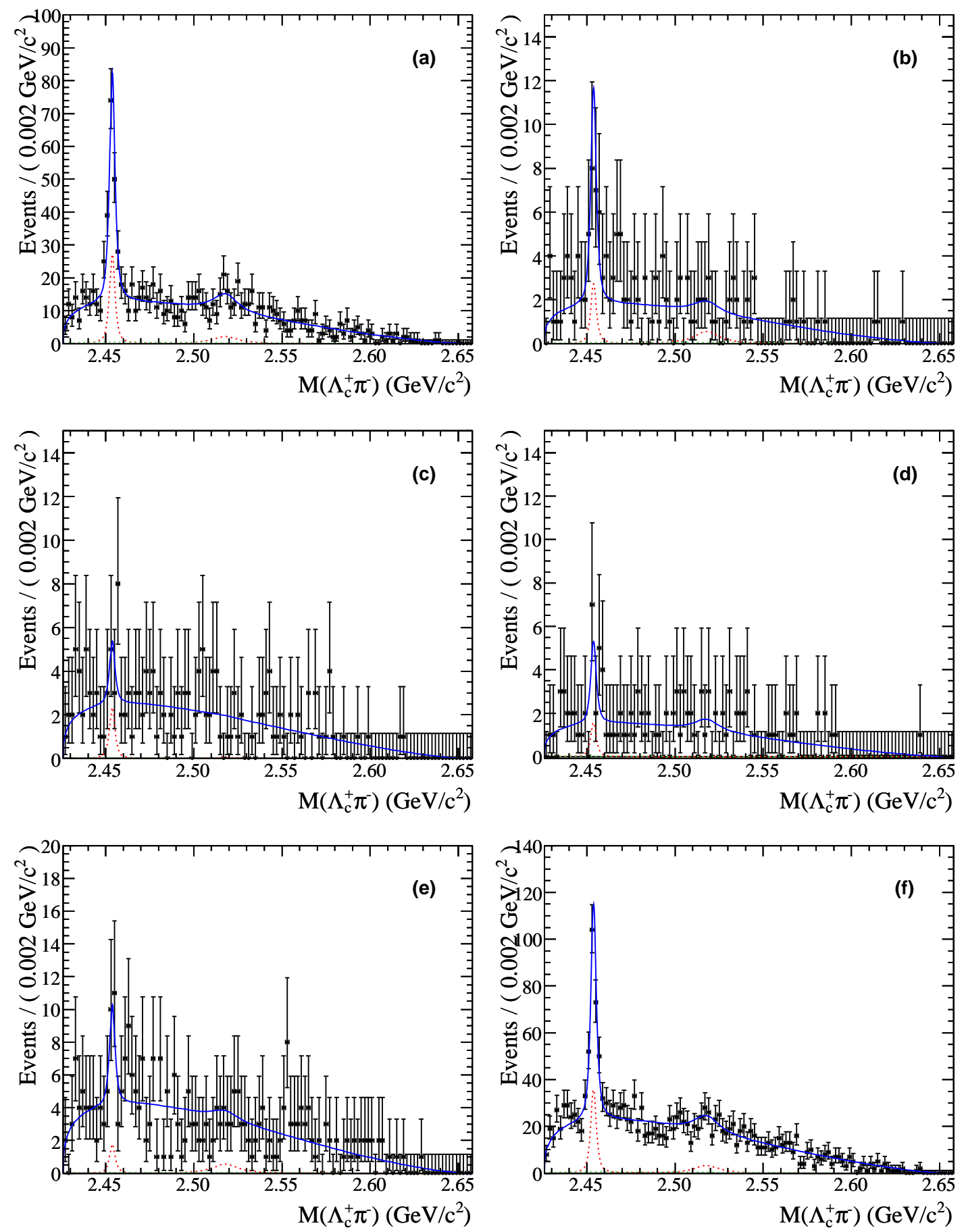

Figure 4.28: $M\left(\Lambda_{c}^{+} \pi^{-}\right)$projections of $\Lambda_{c}^{+} K_{s} \pi^{-}$candidates in data (points with error bars) and their fit (blue curves). The dotted red curves represent the resonant signal components and the dotted green curves represent the non-resonant signal components. The $\Lambda_{c}^{+}$candidates are reconstructed from (a) $p K^{-} \pi^{+}$, (b) $p K_{s}$, (c) $p K_{s} \pi^{-} \pi^{+}$, (d) $\Lambda^{0} \pi^{+}$, (e) $\Lambda^{0} \pi^{+} \pi^{-} \pi^{+}$, and (f) all five final states. 
Table 4.20: Masses, natural widths, yields, and significances of $\Lambda_{c}^{+} K_{s} \pi^{-}$signals.

\begin{tabular}{lcrcc}
\hline & Mass $\left(\mathrm{MeV} / c^{2}\right)$ & Width $(\mathrm{MeV})$ & \multicolumn{1}{c}{ Yield } & Significance \\
\hline \hline$\Xi_{c}(2970)^{0}$ & $2972.9 \pm 4.4 \pm 1.6$ & $31.4 \pm 6.5 \pm 8.2$ & $67 \pm 33 \pm 29$ & $1.7 \sigma$ \\
$\Xi_{c}(3077)^{0}$ & $3079.3 \pm 1.1 \pm 0.2$ & $5.9 \pm 2.3 \pm 1.5$ & $90 \pm 22 \pm 15$ & $4.5 \sigma$ \\
\hline
\end{tabular}

Table 4.21: Yields for $\Xi_{c}(2970)^{0}$ and $\Xi_{c}(3077)^{0}$ three body decays from each of the five $\Lambda_{c}^{+}$decay modes. The errors are statistical and systematic.

\begin{tabular}{lrr}
\hline & $\Xi_{c}(2970)^{0}$ & \multicolumn{1}{c}{$\Xi_{c}(3077)^{0}$} \\
\hline \hline$p K^{-} \pi^{+}$ & $55 \pm 29 \pm 21$ & $59 \pm 16 \pm 11$ \\
$p K_{s}$ & $2 \pm 5 \pm 1$ & $15 \pm 7 \pm 2$ \\
$p K_{s} \pi^{+} \pi^{-}$ & $6 \pm 5 \pm 2$ & $1 \pm 4 \pm 1$ \\
$\Lambda^{0} \pi^{+}$ & $4 \pm 5 \pm 2$ & $0 \pm 3 \pm 1$ \\
$\Lambda^{0} \pi^{+} \pi^{-} \pi^{+}$ & $-1 \pm 5 \pm 3$ & $15 \pm 7 \pm 1$ \\
\hline
\end{tabular}

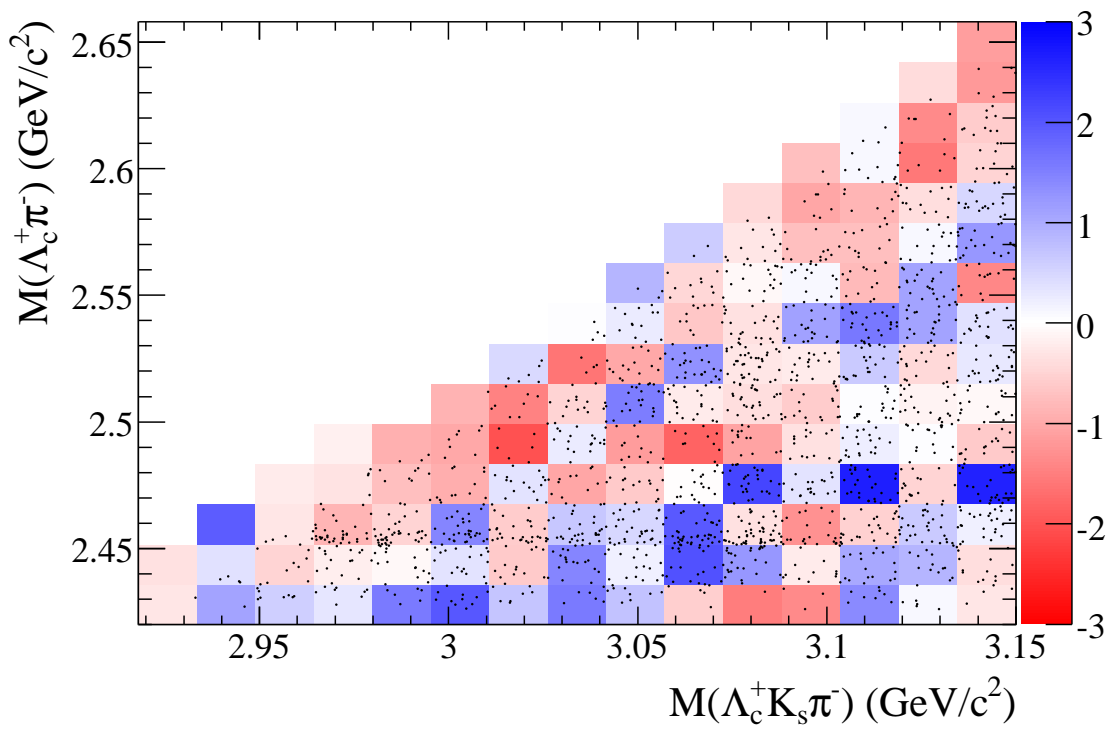

Figure 4.29: Two-dimensional pulls (colored bins) for reconstructed $\Lambda_{c}^{+} K_{s} \pi^{-}$candidates in data (points) and their fit. A $\chi^{2}$ probability of $62 \%$ is calculated for this pull distribution using bins that have at least 11 candidates. 

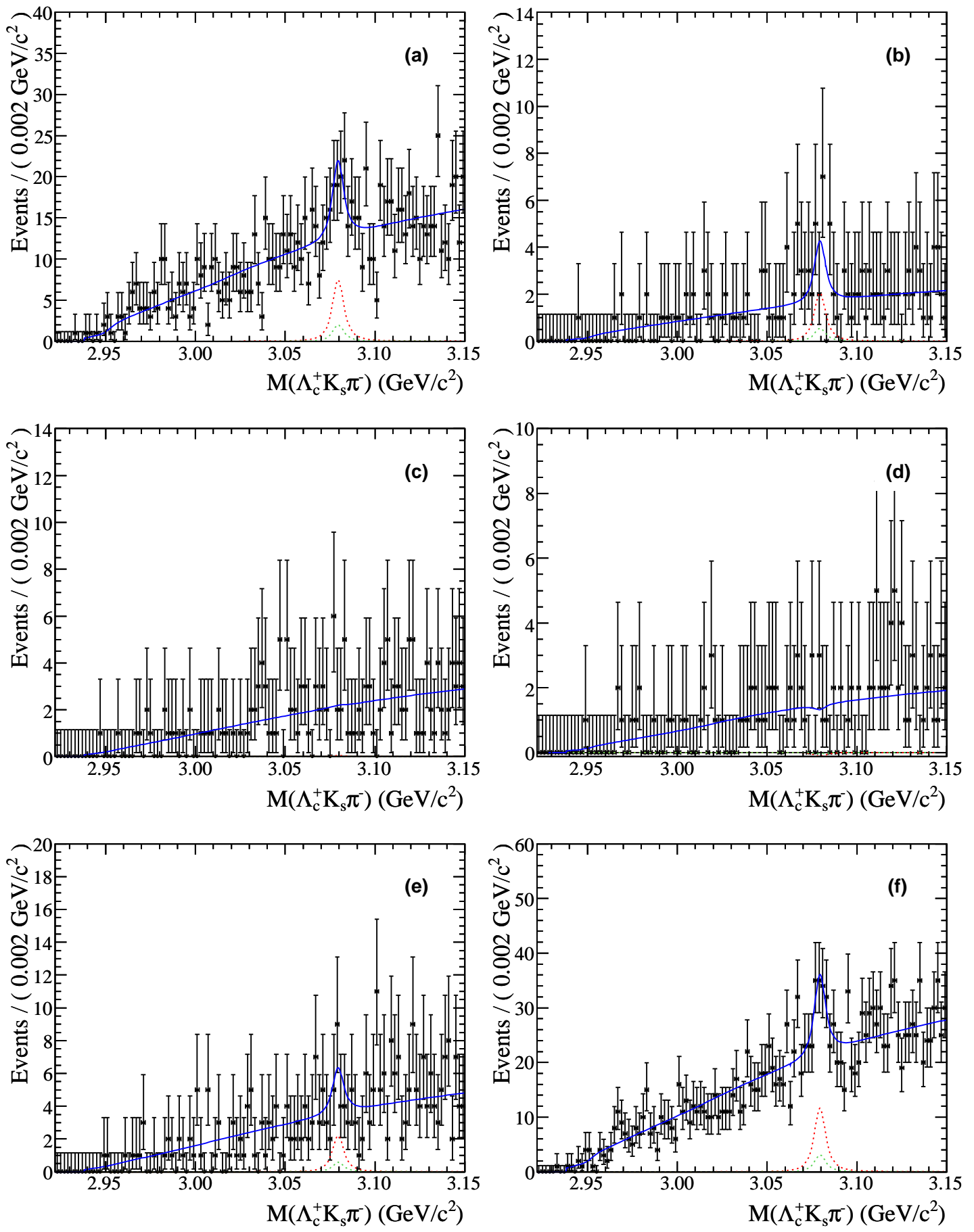

Figure 4.30: $M\left(\Lambda_{c}^{+} K_{s} \pi^{-}\right)$projections of $\Lambda_{c}^{+} K_{s} \pi^{-}$candidates in data (points with error bars) and their fit with background and $\Xi_{c}(3077)^{0} \mathrm{PDF}$ components (blue curves). The dotted red curves represent the resonant signal components and the dotted green curves represent the non-resonant signal components. The $\Lambda_{c}^{+}$candidates are reconstructed from (a) $p K^{-} \pi^{+}$, (b) $p K_{s}$, (c) $p K_{s} \pi^{-} \pi^{+}$, (d) $\Lambda^{0} \pi^{+}$, (e) $\Lambda^{0} \pi^{+} \pi^{-} \pi^{+}$, and (f) all five final states. 

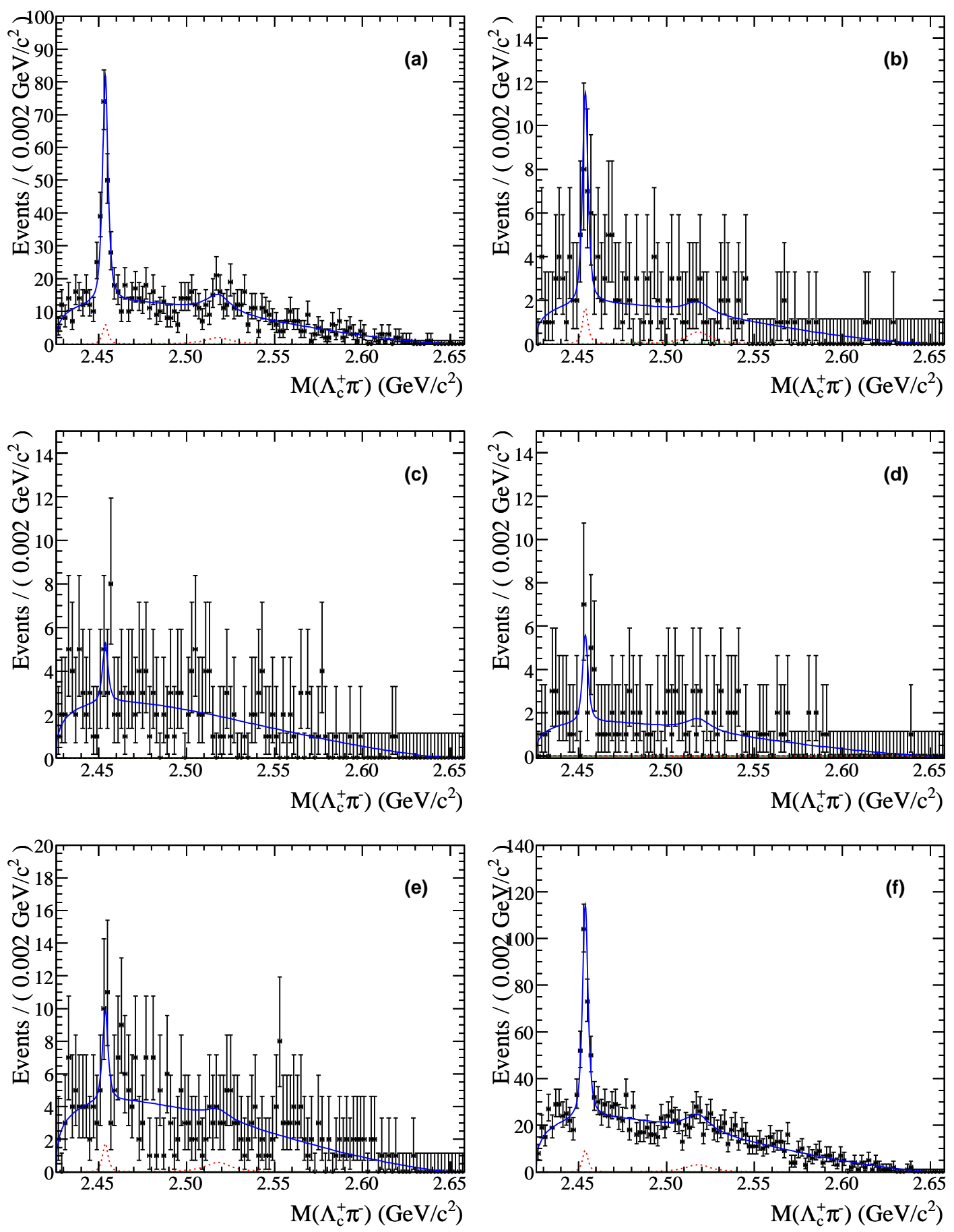

Figure 4.31: $M\left(\Lambda_{c}^{+} \pi^{-}\right)$projections of $\Lambda_{c}^{+} K_{s} \pi^{-}$candidates in data (points with error bars) and their fit with background and $\Xi_{c}(3077)^{0} \mathrm{PDF}$ components (blue curves). The dotted red curves represent the resonant signal components and the dotted green curves represent the non-resonant signal components. The $\Lambda_{c}^{+}$candidates are reconstructed from (a) $p K^{-} \pi^{+}$, (b) $p K_{s}$, (c) $p K_{s} \pi^{-} \pi^{+}$, (d) $\Lambda^{0} \pi^{+}$, (e) $\Lambda^{0} \pi^{+} \pi^{-} \pi^{+}$, and (f) all five final states. 


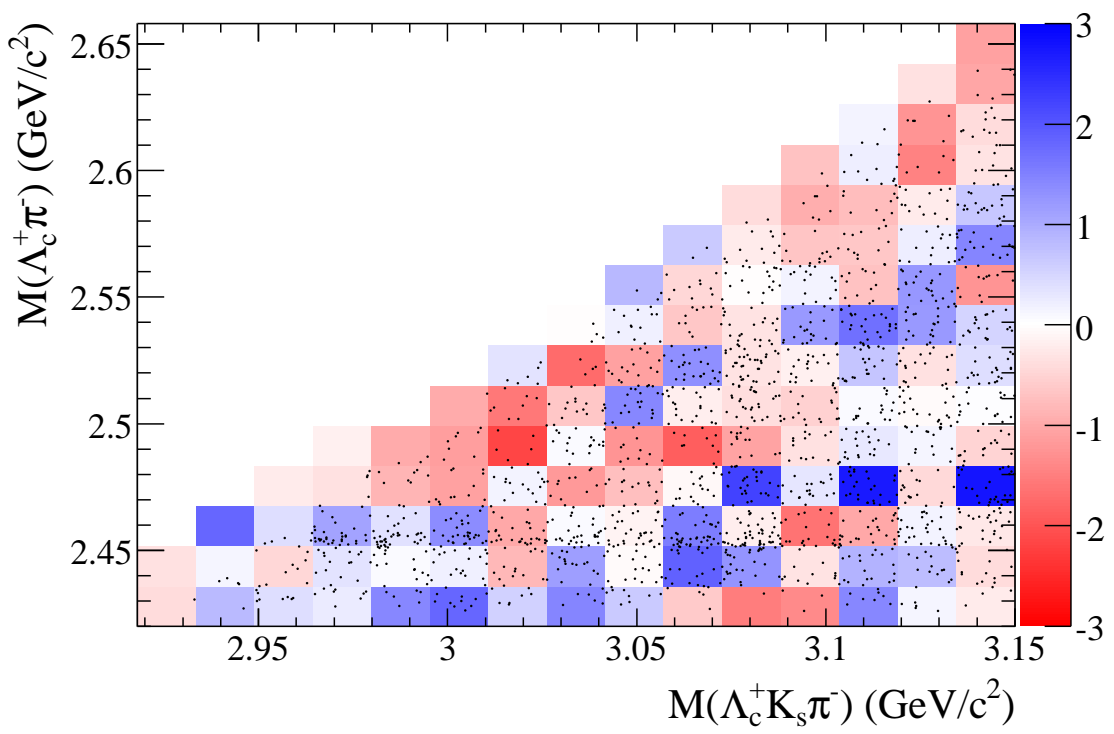

Figure 4.32: Two-dimensional pulls (colored bins) for reconstructed $\Lambda_{c}^{+} K_{s} \pi^{-}$candidates in data (points) and their fit. A $\chi^{2}$ probability of $54 \%$ is calculated for this pull distribution using bins that have at least 11 candidates.

$\Lambda_{c}^{+}$decay mode: $p K^{-} \pi^{+}, p K_{s}, p K_{s} \pi^{-} \pi^{+}, \Lambda^{0} \pi^{+}$, or $\Lambda^{0} \pi^{+} \pi^{-} \pi^{+}$. An unbinned, extended maximumlikelihood technique is used to simultaneously fit all five subsamples using shared signal-PDF shape parameters.

\subsubsection{PDF Components and Likelihood Function}

The PDF component used to describe signal is a double-Voigtian function as detailed by Equation 4.9. For the two-body decay PDF, the means $\mu_{1}$ and $\mu_{2}$ are both fixed at zero. The $\sigma_{1}, \sigma_{2}$, and $f$ parameters have fixed values that are shared among the $\Lambda_{c}^{+}$data subsamples. These values are listed in Table 4.22 .

The PDF component used to describe background for $\Lambda_{c}^{+} K_{s}$ candidates is proportional to a firstorder polynomial. An independent slope parameter is used for each of the $\Lambda_{c}^{+}$decay modes. The PDF component used to describe background for $\Lambda_{c}^{+} K^{-}$candidates is proportional to the function

$$
M_{\Xi_{c}}\left(\frac{M_{\Xi_{c}}^{2}}{T^{2}}-1\right)^{\kappa} \exp \left[\rho \frac{M_{\Xi_{c}}^{2}}{T^{2}}-\rho\right]
$$

where $T$ is the kinematic threshold of $2780.14 \mathrm{MeV} / c^{2}$, and $\kappa$ and $\rho$ are free parameters. The fit range, $2.91 \mathrm{GeV} / c^{2}$ to $3.15 \mathrm{GeV} / c^{2}$, is specifically chosen to be narrow enough to allow for these simple functional forms for the background PDF components while being wide enough to encompass about 
$95 \%$ of the simulated $\Xi_{c}(2970)^{+, 0}$ signals and $99 \%$ of the simulated $\Xi_{c}(3077)^{+, 0}$ signals.

Wrong-sign $\Lambda_{c}^{+} K^{+}$candidates, and right-sign $\Lambda_{c}^{+} K_{s}$ and $\Lambda_{c}^{+} K^{-}$candidates with $\Lambda_{c}^{+}$mass in the sideband regions are used to illustrate and test the functional form of the PDF components describing backgrounds. A fit to the wrong-sign $\Lambda_{c}^{+} K^{+}$data is shown in Figure 4.33. Fits to $\Lambda_{c}^{+}$mass sideband data are shown in Figures 4.34 and 4.35 for $\Lambda_{c}^{+} K_{s}$ and $\Lambda_{c}^{+} K^{-}$candidates, respectively. I use a linear PDF for the fits illustrated in Figures 4.33 through 4.35; no background data sample has an invariant-mass distribution that needs the functional form of Equation 4.18. From the various subfigures, it can be seen that the fitted slope is significantly different between some $\Lambda_{c}^{+}$decay modes. For example, the five fitted slopes in Figure 4.33 are $-1.15 \pm 0.10,-0.65 \pm 0.36,-0.98 \pm 0.33$, $+0.67 \pm 0.46$, and $-1.32 \pm 0.20\left(\mathrm{GeV} / c^{2}\right)^{-1}$. Unlike in the three-body decay modes, the background shape parameters for each $\Lambda_{c}^{+}$mode are independent of each other. For the $\Lambda_{c}^{+} K^{-}$candidates, the background shape parameters of Equation 4.18 are also independent for each $\Lambda_{c}^{+}$decay mode.

The data in each of the $\Lambda_{c}^{+}$-decay modes are simultaneously fit, and the signal-PDF components for each sub-sample share all the same shape parameters. The likelihood function is as discussed in Section 4.3.3 and given by Equations 4.12 and 4.13, but with the search for two-body decays, a Gaussian factor is included to constrain the signal mass and width parameter to those found with the three-body decay analysis. The full likelihood function $\mathcal{L}$ has the form

$$
\mathcal{L}=\exp \left[-\sum_{m=1}^{5}\left(N_{m}-S_{m}-B_{m}\right)\right] \times \exp \left[-\frac{(\mathcal{M}-\mu)^{2}}{2 \sigma_{\mu}^{2}}-\frac{(\Gamma-\gamma)^{2}}{2 \sigma_{\gamma}^{2}}\right] \times \prod_{m=1}^{5} L_{m}\left(S_{m}, B_{m}, \vec{a}, \vec{b} ; x_{m}\right)
$$

where $\mathcal{M}$ and $\Gamma$ are the mass and width of the signal as measured from the fit to three-body decay data, and $\mu$ and $\gamma$ are the signal mass and width parameters from the two-body fit.

The significance of a signal is measured using the change in likelihood when the signal PDF component is removed from the likelihood function and the data is refit. Twice this change in likelihood is equivalent to a $\Delta \chi^{2}$ for the joint estimation of seven parameters (mass, width, and a yield from each of the five $\Lambda_{c}^{+}$decay modes). A one-sided Gaussian significance is calculated from the $\chi^{2}$ probability with seven degrees of freedom. In the absence of a significant signal, an upper limit on the number of possible signal candidates is calculated by integrating the maximized-likelihood distribution for positive yield. When calculating these upper limits, the ratios of yields between the five used $\Lambda_{c}^{+}$decay modes are Gaussian constrained based on estimated efficiency ratios, and worldaverage $\Lambda_{c}^{+}$branching ratios and their errors [7]. The upper limits cross-sections times branching fractions are calculated based on upper limits on yield. 

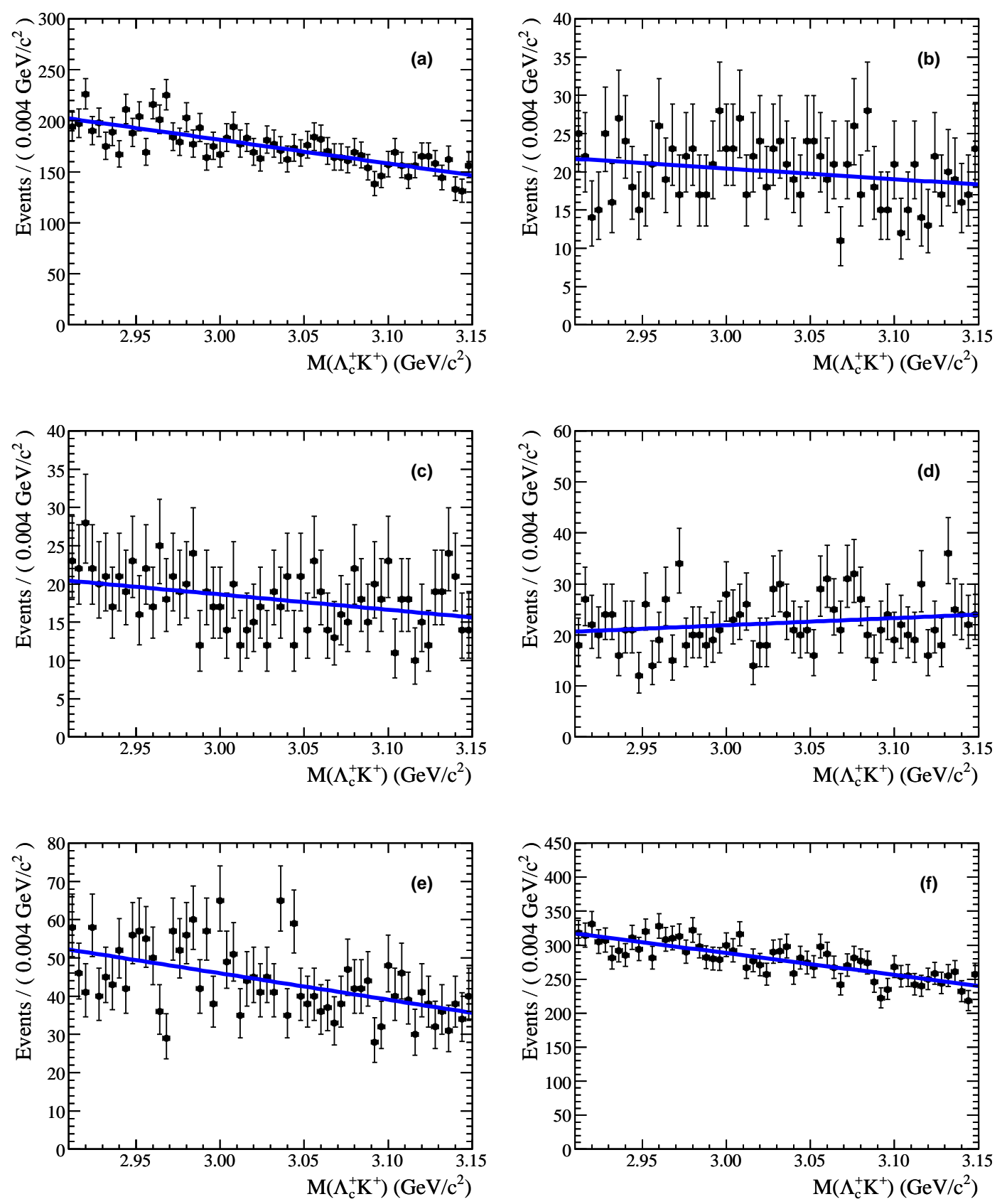

Figure 4.33: Invariant-mass distributions of wrong-sign $\Lambda_{c}^{+} K^{+}$candidates (points with error bars). The blue lines represent the linear fit to the data. The $\Lambda_{c}^{+}$candidates are reconstructed from (a) $p K^{-} \pi^{+}$, (b) $p K_{s}$, (c) $p K_{s} \pi^{-} \pi^{+}$, (d) $\Lambda^{0} \pi^{+}$, (e) $\Lambda^{0} \pi^{+} \pi^{-} \pi^{+}$, and (f) all five final states. 

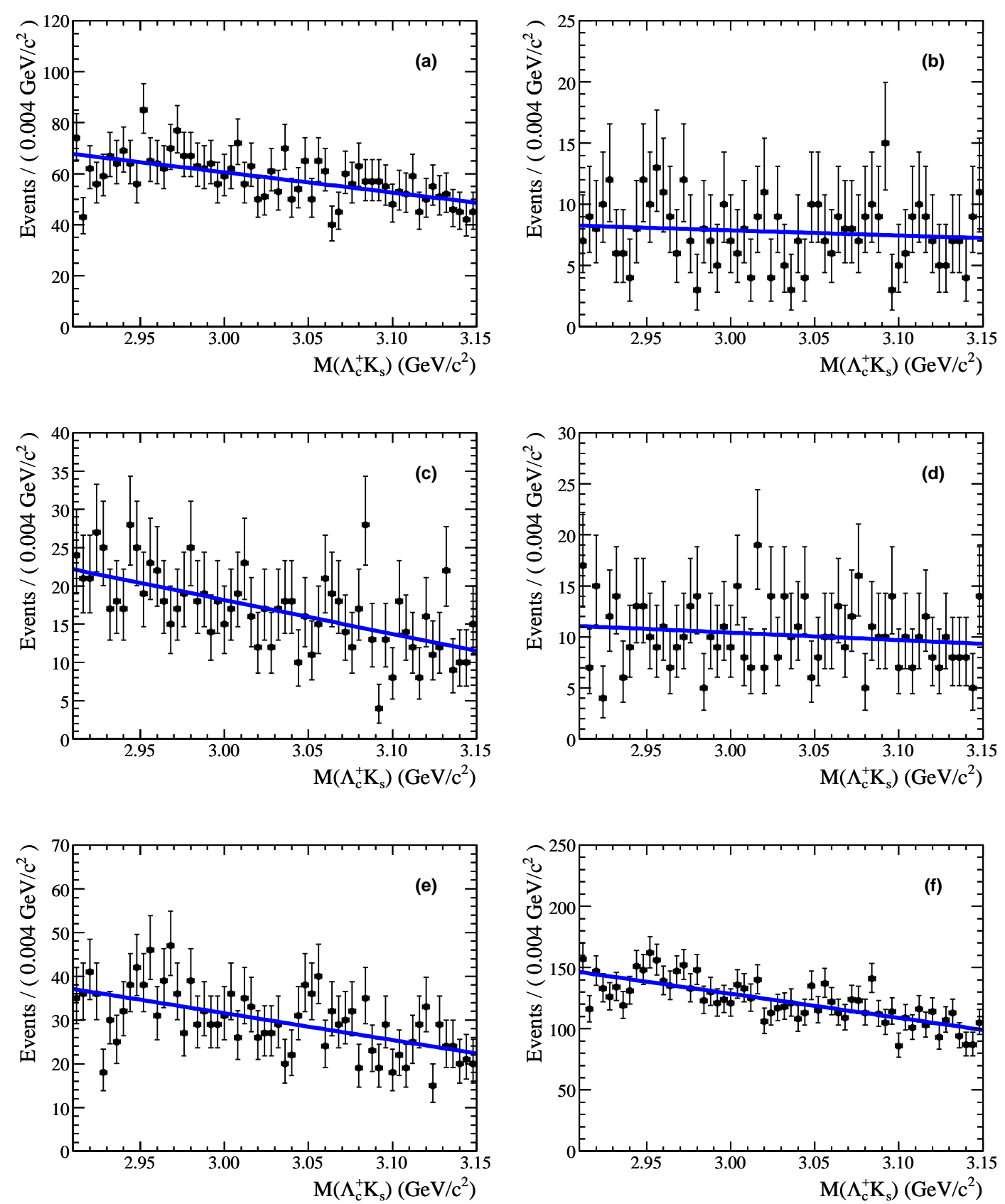

Figure 4.34: Invariant-mass distributions of $\Lambda_{c}^{+} K_{s}$ candidates with $\Lambda_{c}^{+}$mass in the sideband range (points with error bars). The blue lines represent the linear fit to the data. The $\Lambda_{c}^{+}$candidates are reconstructed from (a) $p K^{-} \pi^{+}$, (b) $p K_{s}$, (c) $p K_{s} \pi^{-} \pi^{+}$, (d) $\Lambda^{0} \pi^{+}$, (e) $\Lambda^{0} \pi^{+} \pi^{-} \pi^{+}$, and (f) all five final states. 

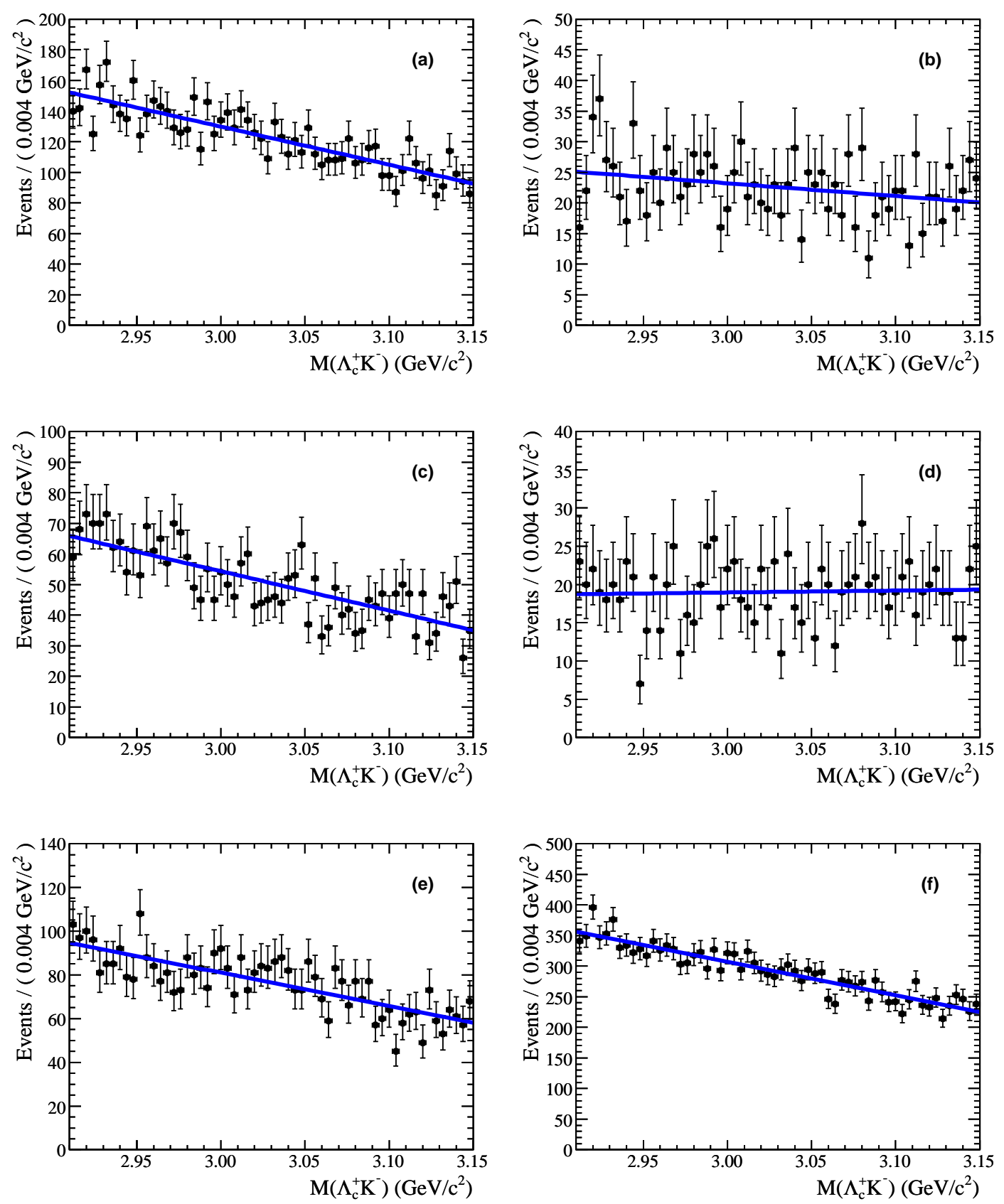

Figure 4.35: Invariant-mass distributions of $\Lambda_{c}^{+} K^{-}$candidates with $\Lambda_{c}^{+}$mass in the sideband range (points with error bars). The blue lines represent the linear fit to the data. The $\Lambda_{c}^{+}$candidates are reconstructed from (a) $p K^{-} \pi^{+}$, (b) $p K_{s}$, (c) $p K_{s} \pi^{-} \pi^{+}$, (d) $\Lambda^{0} \pi^{+}$, (e) $\Lambda^{0} \pi^{+} \pi^{-} \pi^{+}$, and (f) all five final states. 
Table 4.22: $M\left(\Lambda_{c}^{+} K_{s}\right)$ and $M\left(\Lambda_{c}^{+} K^{-}\right)$mass resolutions for two-body decay modes. $\sigma_{N}$ refers to the width of the narrower of the two Gaussians; $\sigma_{W}$ refers to the wider of the two. Fraction refers to the relative fraction of the narrower Gaussian.

\begin{tabular}{lccc}
\hline Signal & $\sigma_{N}\left(\mathrm{MeV} / c^{2}\right)$ & $\sigma_{W}\left(\mathrm{MeV} / c^{2}\right)$ & Fraction $(\%)$ \\
\hline \hline$\Xi_{c}(2970)^{+}$ & $1.83 \pm 0.02$ & $4.35 \pm 0.16$ & $85 \pm 2$ \\
$\Xi_{c}(3055)^{+}$ & $2.03 \pm 0.03$ & $4.14 \pm 0.14$ & $76 \pm 2$ \\
$\Xi_{c}(3077)^{+}$ & $2.17 \pm 0.03$ & $4.52 \pm 0.16$ & $79 \pm 2$ \\
$\Xi_{c}(3123)^{+}$ & $2.19 \pm 0.05$ & $4.58 \pm 0.17$ & $71 \pm 3$ \\
\hline \hline
\end{tabular}

Table 4.23: Estimated efficiencies, given in units of $10^{-4}$, for two-body decay modes. Efficiencies are calculated separately for each of the five simulated $\Lambda_{c}^{+}$decay modes. The first errors are statistical and the second errors are systematic.

\begin{tabular}{lrrrrr}
\hline & \multicolumn{1}{c}{$p K^{-} \pi^{+}$} & \multicolumn{1}{c}{$p K_{s}$} & \multicolumn{1}{c}{$p K_{s} \pi^{-} \pi^{+}$} & \multicolumn{1}{c}{$\Lambda^{0} \pi^{+}$} & \multicolumn{1}{c}{$\Lambda^{0} \pi^{+} \pi^{-} \pi^{+}$} \\
\hline \hline$\Xi_{c}(2970)^{0}$ & $1110 \pm 8 \pm 52$ & $321 \pm 6 \pm 16$ & $151 \pm 4 \pm 8$ & $547 \pm 13 \pm 31$ & $264 \pm 5 \pm 15$ \\
$\Xi_{c}(3077)^{0}$ & $1188 \pm 9 \pm 56$ & $322 \pm 7 \pm 16$ & $155 \pm 4 \pm 9$ & $594 \pm 14 \pm 33$ & $277 \pm 5 \pm 16$ \\
$\Xi_{c}(2970)^{+}$ & $227 \pm 2 \pm 12$ & $75 \pm 2 \pm 5$ & $37 \pm 1 \pm 3$ & $138 \pm 4 \pm 9$ & $64 \pm 2 \pm 5$ \\
$\Xi_{c}(3055)^{+}$ & $251 \pm 3 \pm 13$ & $86 \pm 2 \pm 5$ & $36 \pm 1 \pm 3$ & $148 \pm 5 \pm 10$ & $69 \pm 2 \pm 5$ \\
$\Xi_{c}(3077)^{+}$ & $246 \pm 2 \pm 13$ & $82 \pm 2 \pm 5$ & $39 \pm 1 \pm 3$ & $146 \pm 4 \pm 10$ & $65 \pm 2 \pm 5$ \\
$\Xi_{c}(3123)^{+}$ & $261 \pm 3 \pm 13$ & $88 \pm 2 \pm 6$ & $36 \pm 1 \pm 3$ & $153 \pm 5 \pm 10$ & $71 \pm 2 \pm 5$ \\
\hline \hline
\end{tabular}

\subsubsection{Mass Resolution and Signal Efficiency}

I determine the $M\left(\Lambda_{c}^{+} K_{s}\right)$ and $M\left(\Lambda_{c}^{+} K^{-}\right)$mass resolutions by studying the difference between the simulated mass and the reconstructed mass in signal-MC samples. These mass-difference distributions are fit with two Gaussian functions with a common mean. I find no evidence for significant resolution differences between the five $\Lambda_{c}^{+}$decay modes. The measurements from the five $\Lambda_{c}^{+}$decay modes areaveraged and listed in Table 4.22. The averaged Gaussian widths and relative fractions are used for the double-Voigtian signal PDF components.

The efficiencies for finding the two-body signal decays are determined with signal-MC studies. In these studies the invariant-mass distributions for signal-MC candidates are fit with the doubleVoigtian PDF shapes. The $M\left(\Lambda_{c}^{+} K_{s}\right)$ and $M\left(\Lambda_{c}^{+} K^{-}\right)$invariant mass ranges in these studies are restricted to the two-body search region. The fits used for estimating $\Xi_{c}(2970)^{+}$and $\Xi_{c}(3077)^{+}$ efficiencies are shown if Figures 4.36 and 4.37, respectively. Similar fits are performed for $\Xi_{c}(2970)^{0}$, $\Xi_{c}(3077)^{0}, \Xi_{c}(3055)^{+}$, and $\Xi_{c}(3123)^{+}$. To calculate each efficiency, the number of fitted signal$\mathrm{MC}$ events is divided by the number of signal-MC events generated. The efficiencies are listed in Table 4.23 with the first errors being statistical and the second errors being systematic. 

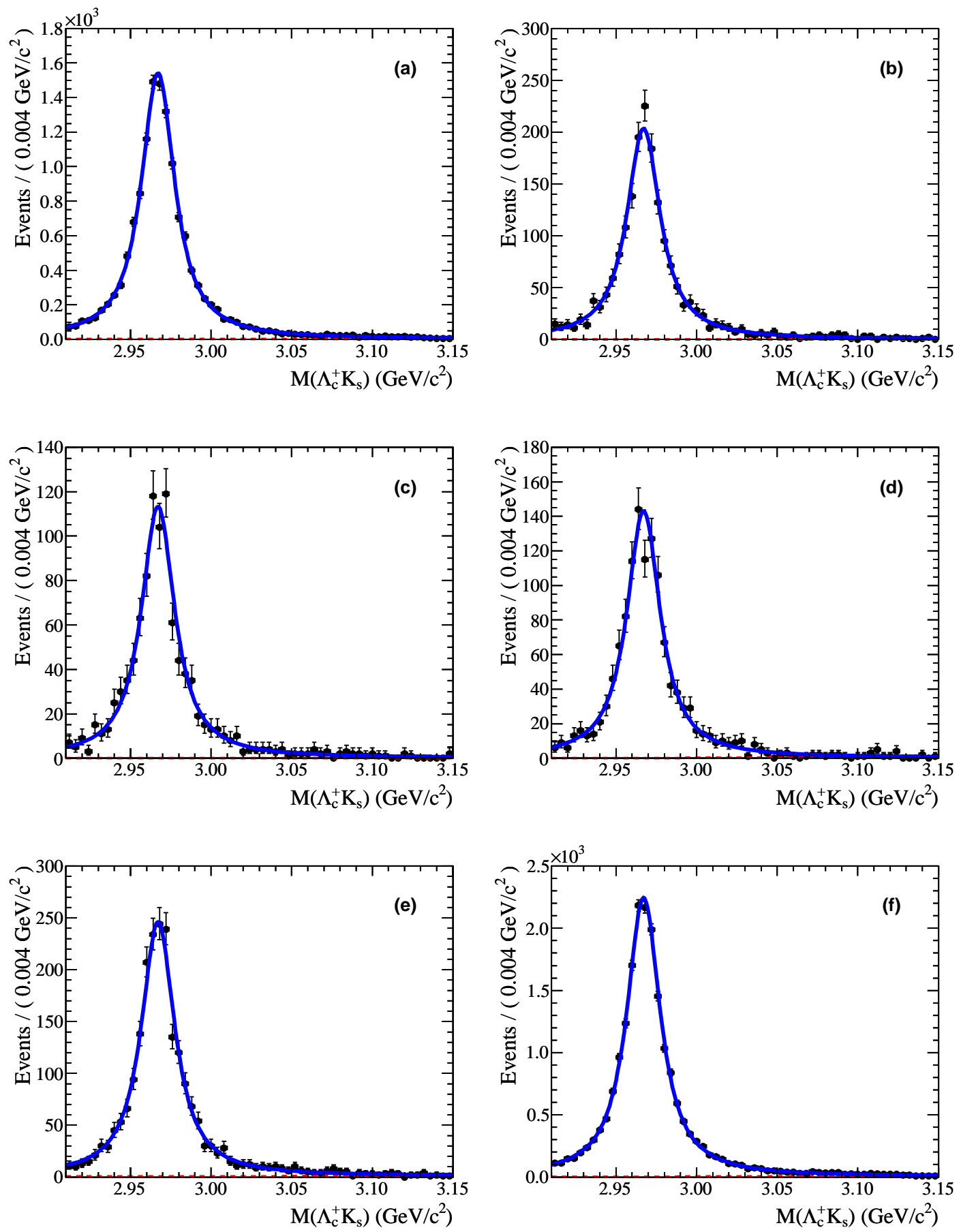

Figure 4.36: Unbinned likelihood fits to signal-MC $\Xi_{c}(2970)^{+} \rightarrow \Lambda_{c}^{+} K_{s}$ candidates used for estimating efficiencies. The blue curves represent the double-Voigtian plus background shape fits to the signal-MC samples (points with error bars) where the $\Lambda_{c}^{+}$decays to (a) $p K^{-} \pi^{+}$, (b) $p K_{s}$, (c) $p K_{s} \pi^{-} \pi^{+}$, (d) $\Lambda^{0} \pi^{+}$, (e) $\Lambda^{0} \pi^{+} \pi^{-} \pi^{+}$, or (f) any of these five final states. The red dashed lines indicate the fitted linear (or constant) background components. 

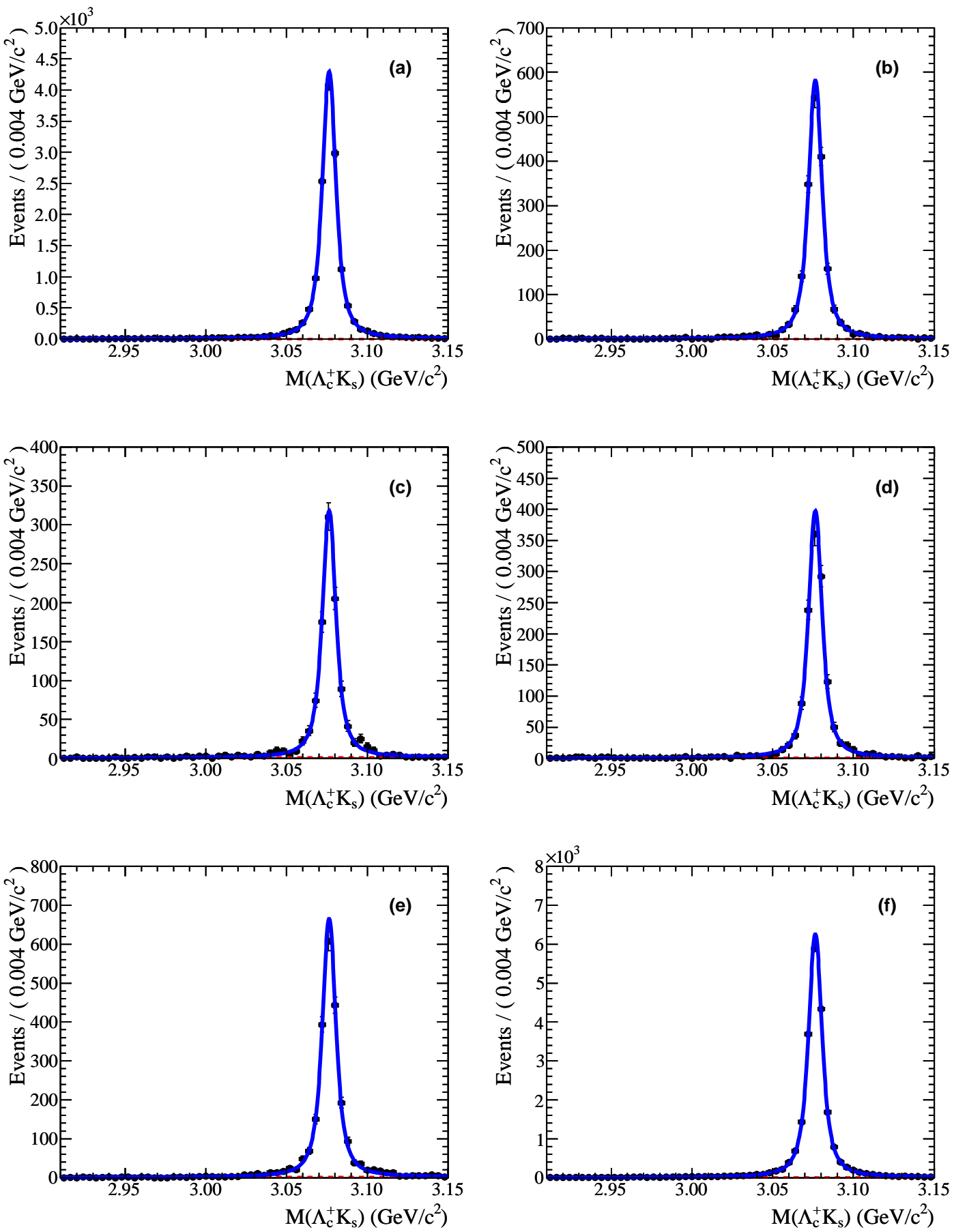

Figure 4.37: Unbinned likelihood fits to signal-MC $\Xi_{c}(3077)^{+} \rightarrow \Lambda_{c}^{+} K_{s}$ candidates used for estimating efficiencies. The blue curves represent the double-Voigtian plus background shape fits to the signal-MC samples (points with error bars) where the $\Lambda_{c}^{+}$decays to (a) $p K^{-} \pi^{+}$, (b) $p K_{s}$, (c) $p K_{s} \pi^{-} \pi^{+}$, (d) $\Lambda^{0} \pi^{+}$, (e) $\Lambda^{0} \pi^{+} \pi^{-} \pi^{+}$, or (f) any of these five final states. The red dashed lines indicate the fitted linear (or constant) background components. 
Table 4.24: Results for two-body decay modes. All quantities are 90\% CL upper limits. The columns are labels with the symbols $\sigma_{Y}=\sigma\left(e^{+} e^{-} \rightarrow Y X\right), \mathcal{B}_{Z}=\mathcal{B}\left(Y \rightarrow \Lambda_{c}^{+} Z\right)$, and $\mathcal{B}_{\Lambda_{c}}=$ $\mathcal{B}\left(\Lambda_{c}^{+} \rightarrow p K^{-} \pi^{+}\right)$.

\begin{tabular}{crrcc}
\hline$Y$ & $Z$ & Yield & $\sigma_{Y} \mathcal{B}_{Z} \mathcal{B}_{\Lambda_{c}}$ & $\frac{\mathcal{B}\left(Y \rightarrow \Lambda_{c}^{+} Z\right)}{\mathcal{B}\left(Y \rightarrow \Lambda_{c}^{+} K^{-} \pi^{+}\right)}$ \\
\hline \hline$\Xi_{c}(2970)^{0}$ & $K^{-}$ & 415 & $9.7 \mathrm{fb}$ & 2.3 \\
$\Xi_{c}(3077)^{0}$ & $K^{-}$ & 57 & $1.2 \mathrm{fb}$ & 0.2 \\
$\Xi_{c}(2970)^{+}$ & $K_{s}$ & 104 & $12 \mathrm{fb}$ & 1.1 \\
$\Xi_{c}(3055)^{+}$ & $K_{s}$ & 83 & $8.6 \mathrm{fb}$ & 3.9 \\
$\Xi_{c}(3077)^{+}$ & $K_{s}$ & 27 & $2.9 \mathrm{fb}$ & 0.35 \\
$\Xi_{c}(3123)^{+}$ & $K_{s}$ & 27 & $2.7 \mathrm{fb}$ & 1.7 \\
\hline \hline
\end{tabular}

\subsubsection{Data Results}

Both the $\Lambda_{c}^{+} K_{s}$ and the $\Lambda_{c}^{+} K^{-}$invariant-mass distributions do not exhibit evidence for any statistically significant peaking signal. Figures 4.38 and 4.39 show fits to the $\Lambda_{c}^{+} K_{s}$ invariant-mass distribution with PDF compnents corresponding to $\Xi_{c}(2970)^{+}$and $\Xi_{c}(3077)^{+}$, respectively. Figures 4.40 and 4.41 show fits to the $\Lambda_{c}^{+} K_{s}$ invariant-mass distribution with PDF compnents corresponding to $\Xi_{c}(2970)^{0}$ and $\Xi_{c}(3077)^{0}$, respectively.

As no significant signals are found, $90 \%$ confidence-level (CL) upper limits are determined for signal yields and their corresponding cross-sections times branching fractions. The upper limits on cross-sections times branching fractions are also presented relative to their corresponding three-body decay mode measurements. All results are listed in Table 4.24.

\subsection{Four-Body Decays}

The search for excited charm-strange baryons decaying to $\Lambda_{c}^{+} K_{s} \pi^{+} \pi^{-}$and $\Lambda_{c}^{+} K^{-} \pi^{+} \pi^{-}$is described in this section. I search for signal up to $250 \mathrm{MeV} / c^{2}$ above the kinematic lower limits of the invariant masses $M\left(\Lambda_{c}^{+} K_{s} \pi^{+} \pi^{-}\right)$and $M\left(\Lambda_{c}^{+} K^{-} \pi^{+} \pi^{-}\right)$. Because the $\Xi_{c}(3077)^{+, 0}$ mass is only about $15 \mathrm{MeV} / c^{2}$ above the kinematic threshold for these four-body decays, no intermediate resonant states are possible and none are searched for. The data are divided into five subsamples based on the reconstructed $\Lambda_{c}^{+}$decay mode: $p K^{-} \pi^{+}, p K_{s}, p K_{s} \pi^{-} \pi^{+}, \Lambda^{0} \pi^{+}$, or $\Lambda^{0} \pi^{+} \pi^{-} \pi^{+}$. An unbinned, extended maximum-likelihood technique is used to simultaneously fit each subsample of data using shared PDF shape parameters. 

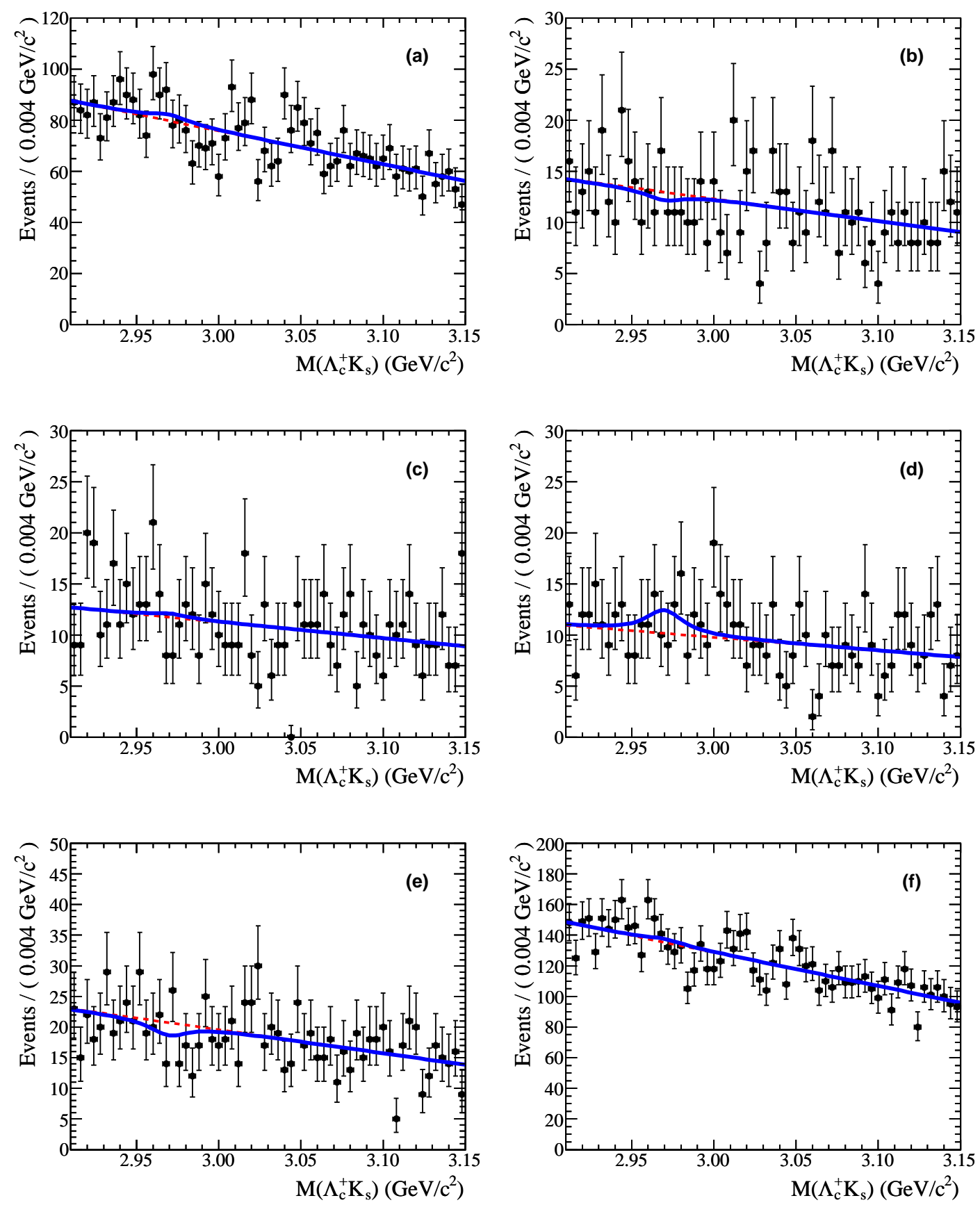

Figure 4.38: $\Lambda_{c}^{+} K_{s}$ candidates (points with error bars) and fit with a PDF component for a $\Xi_{c}(2970)^{+}$signal. The blue lines represent the total fit to the data, and the dashed red lines represent the background fit. The $\Lambda_{c}^{+}$is reconstructed in decays to (a) $p K^{-} \pi^{+}$, (b) $p K_{s}$, (c) $p K_{s} \pi^{-} \pi^{+}$, (d) $\Lambda^{0} \pi^{+}$, (e) $\Lambda^{0} \pi^{+} \pi^{-} \pi^{+}$, or (f) any of these five final states. 

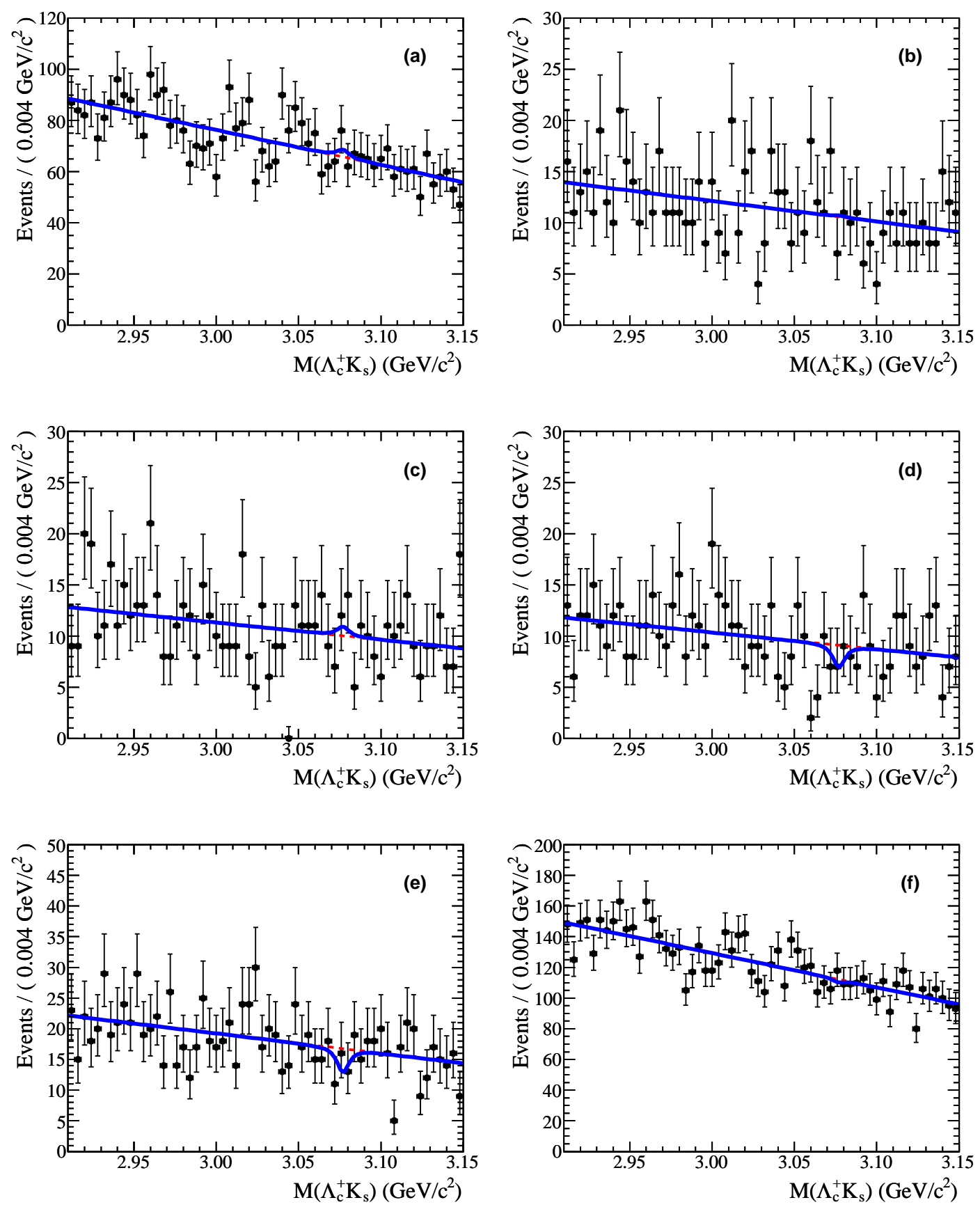

Figure 4.39: $\Lambda_{c}^{+} K_{s}$ candidates (points with error bars) and fit with a PDF component for a $\Xi_{c}(3077)^{+}$signal. The blue lines represent the total fit to the data, and the dashed red lines represent the background fit. The $\Lambda_{c}^{+}$is reconstructed in decays to (a) $p K^{-} \pi^{+}$, (b) $p K_{s}$, (c) $p K_{s} \pi^{-} \pi^{+}$, (d) $\Lambda^{0} \pi^{+}$, (e) $\Lambda^{0} \pi^{+} \pi^{-} \pi^{+}$, or (f) any of these five final states. 

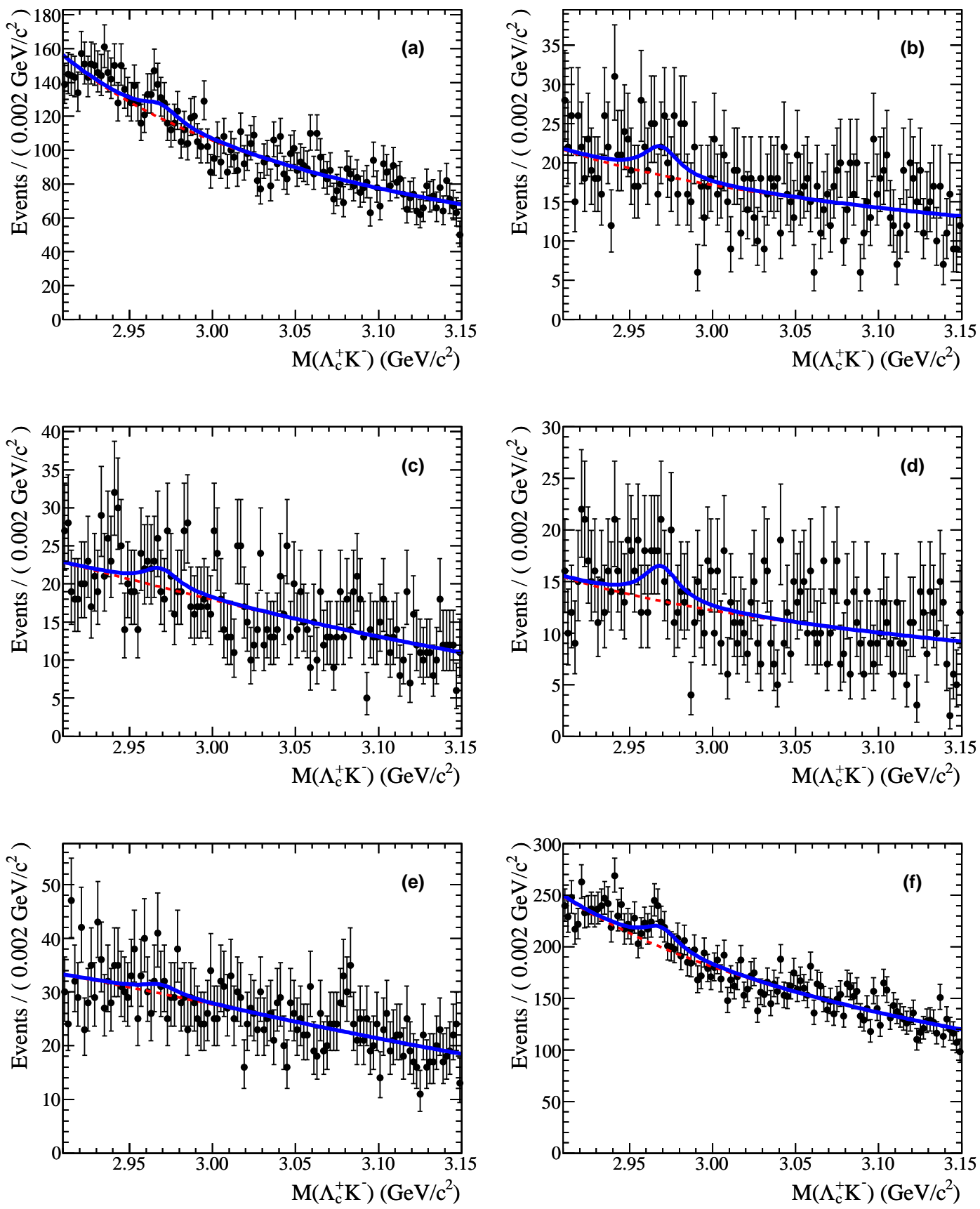

Figure 4.40: $\Lambda_{c}^{+} K^{-}$candidates (points with error bars) and fit with a PDF component for a $\Xi_{c}(2970)^{0}$ signal. The blue lines represent the total fit to the data, and the dashed red lines represent the background fit. The $\Lambda_{c}^{+}$is reconstructed in decays to (a) $p K^{-} \pi^{+}$, (b) $p K_{s}$, (c) $p K_{s} \pi^{-} \pi^{+}$, (d) $\Lambda^{0} \pi^{+}$, (e) $\Lambda^{0} \pi^{+} \pi^{-} \pi^{+}$, or (f) any of these five final states. 

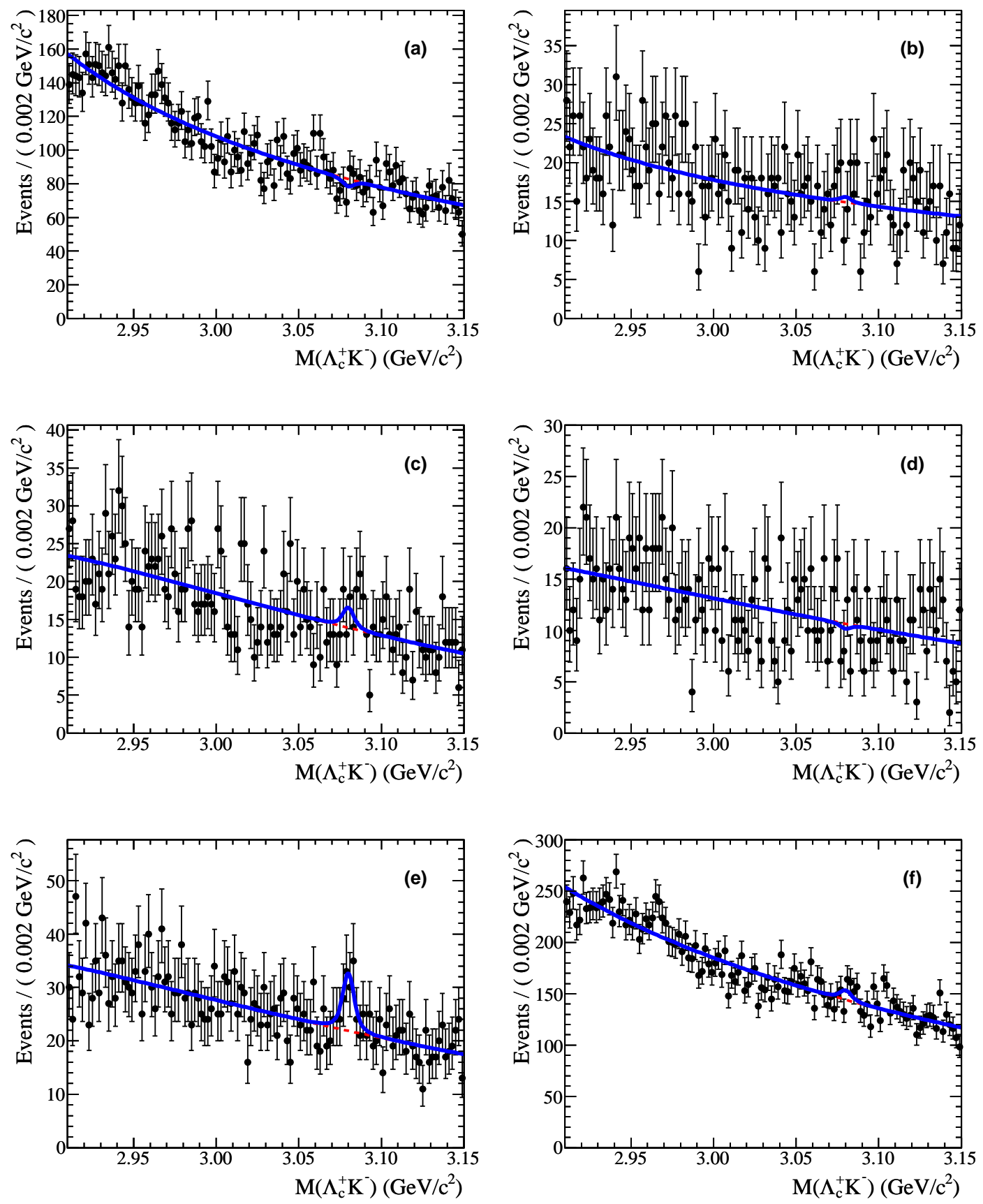

Figure 4.41: $\Lambda_{c}^{+} K^{-}$candidates (points with error bars) and fit with a PDF component for a $\Xi_{c}(3077)^{0}$ signal. The blue lines represent the total fit to the data, and the dashed red lines represent the background fit. The $\Lambda_{c}^{+}$is reconstructed in decays to (a) $p K^{-} \pi^{+}$, (b) $p K_{s}$, (c) $p K_{s} \pi^{-} \pi^{+}$, (d) $\Lambda^{0} \pi^{+}$, (e) $\Lambda^{0} \pi^{+} \pi^{-} \pi^{+}$, or (f) any of these five final states. 

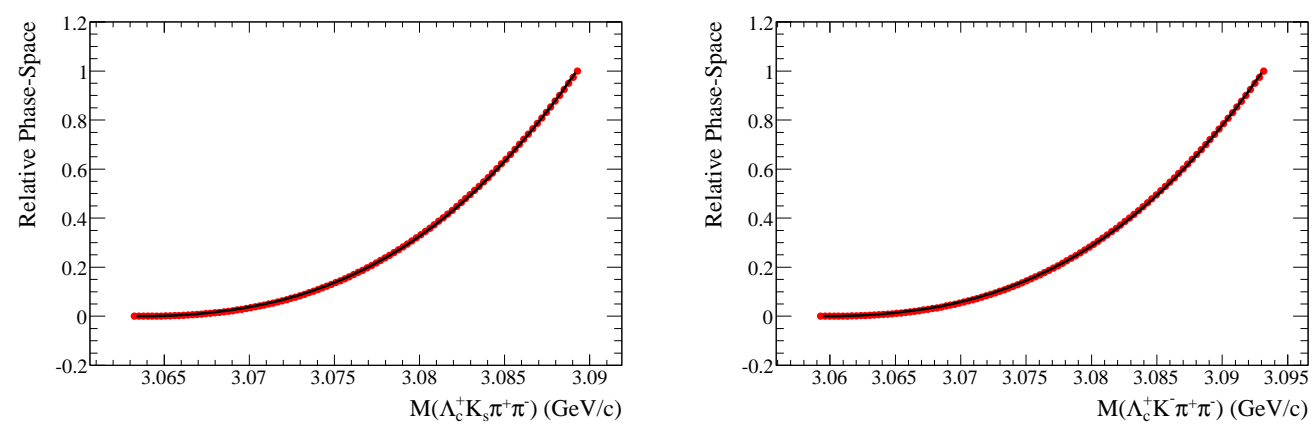

Figure 4.42: Relative four-body phase-space Figure 4.43: Relative four-body phase-space as a function of the $\Lambda_{c}^{+} K_{s} \pi^{+} \pi^{-}$invariant as a function of the $\Lambda_{c}^{+} K^{-} \pi^{+} \pi^{-}$invariant mass (red points) with a third-order polyno- mass (red points) with a third-order polynomial fit (black curve). mial fit (black curve).

\subsubsection{PDF Components and Likelihood Function}

The PDF component used to describe signal is is a double-Voigtian function (as described by Equation 4.9) multiplied by a four-body phase-space function. The four-body phase-space function describes the relative volume of available phase space for the four-body decay as a function of the excited charm-strange baryon's invariant mass. The relative volume of phase space is numerically calculated and its distribution is fit with the third-order polynomial. This third-order polynomials is used as the four-body phase-space function. The numerically calculated relative phase-space and fit shown in Figures 4.42 and 4.43 for $\Lambda_{c}^{+} K_{s} \pi^{+} \pi^{-}$and $\Lambda_{c}^{+} K^{-} \pi^{+} \pi^{-}$, respectively. The PDF component used to fit data is proportional to a third-order polynomial and has one free parameter.

Wrong-sign $\Lambda_{c}^{+} K^{+} \pi^{-} \pi^{-}$candidates, and right-sign $\Lambda_{c}^{+} K_{s} \pi^{+} \pi^{-}$candidates with $\Lambda_{c}^{+}$mass in the sideband region are used to illustrate and test the functional form of the PDF components describing background. Fits to these distributions are shown in Figures 4.44 and 4.45 . These background distributions are well described by the background components of the PDFs.

The likelihood function is constructed in the same manner as described in Section 4.3.3 and Equation 4.13. The likelihood function is extended as described in Section 4.4.1 to give the signal mass and width parameters Gaussian constraints to their measured values from the corresponding signals with three-body decay. In the absence of a significant signal, an upper limit on the number of possible signal candidates is calculated by integrating the maximized-likelihood distribution for positive yield. When calculating these upper limits, the ratios of yields between the five used $\Lambda_{c}^{+}$ decay modes are Gaussian constrained based on estimated efficiency ratios and world-average $\Lambda_{c}^{+}$ branching ratios and their errors [7]. The upper limits cross-sections times branching fractions are calculated based on upper limits on yield. 

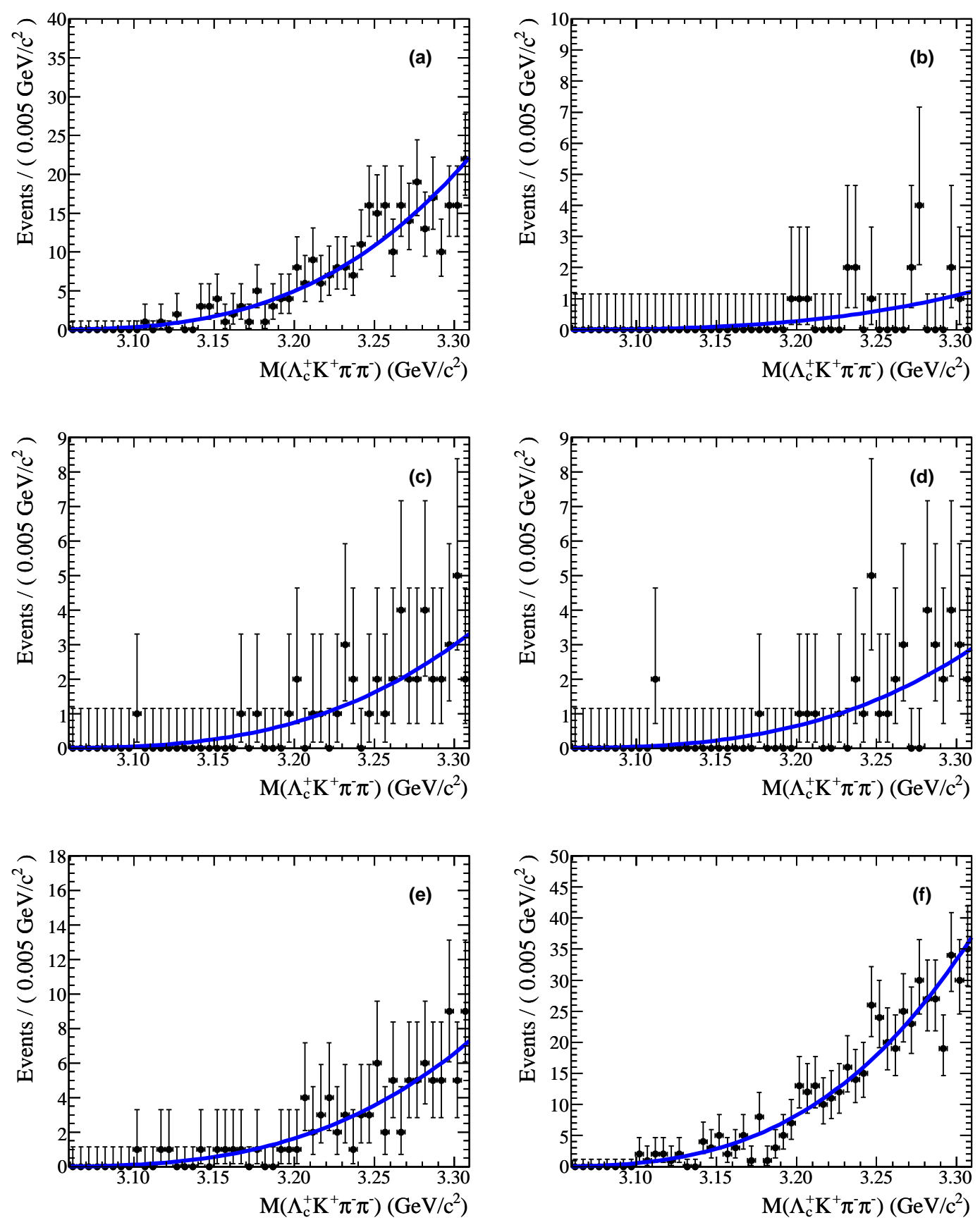

Figure 4.44: Invariant-mass distributions of wrong-sign $\Lambda_{c}^{+} K^{+} \pi^{-} \pi^{-}$candidates (points with error bars). The blue lines represent the background PDF. The $\Lambda_{c}^{+}$candidates are reconstructed from (a) $p K^{-} \pi^{+}$, (b) $p K_{s}$, (c) $p K_{s} \pi^{-} \pi^{+}$, (d) $\Lambda^{0} \pi^{+}$, (e) $\Lambda^{0} \pi^{+} \pi^{-} \pi^{+}$, and (f) all five final states. 

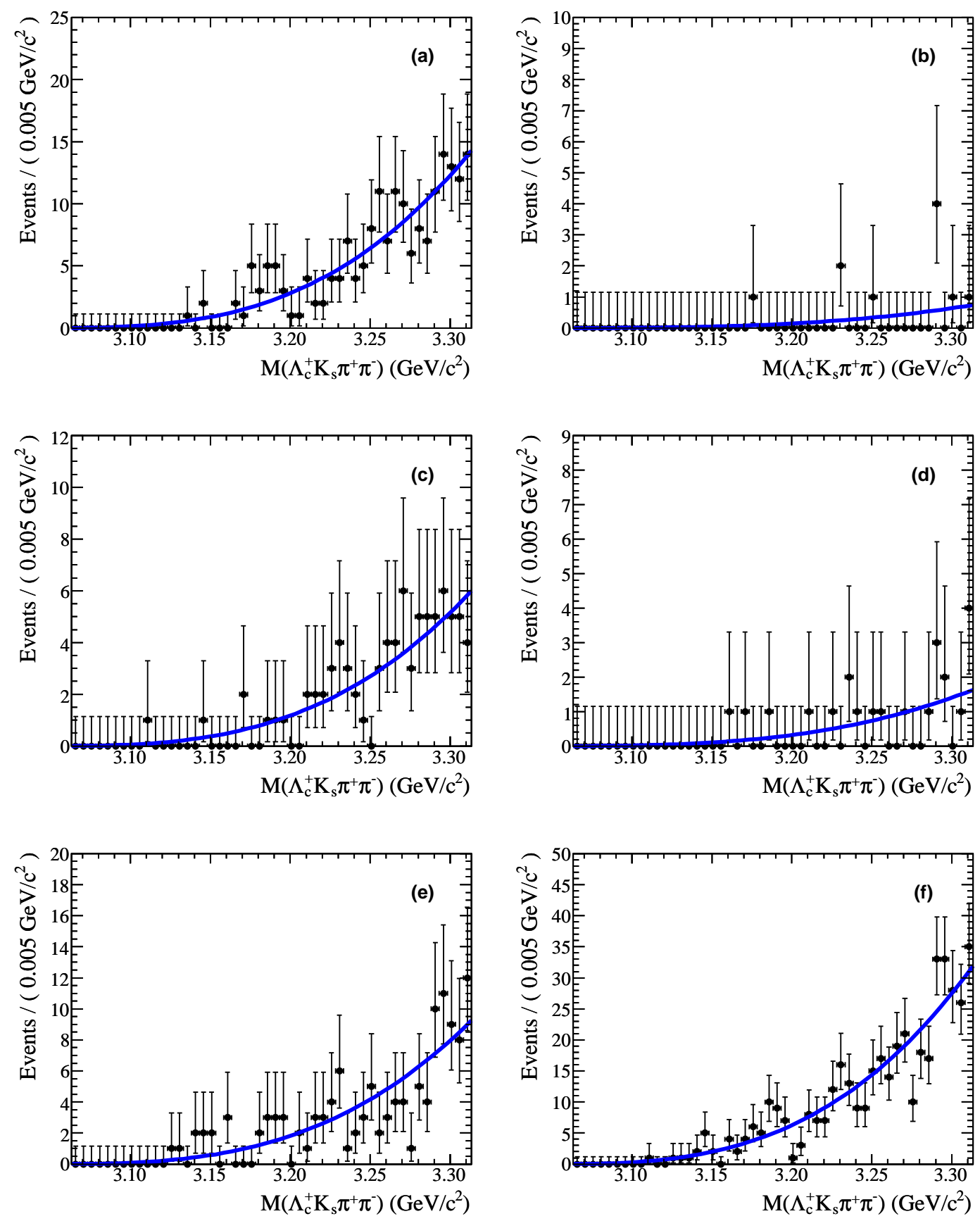

Figure 4.45: Invariant-mass distributions of $\Lambda_{c}^{+}$side-band $\Lambda_{c}^{+} K^{-} \pi^{-} \pi^{+}$candidates (points with error bars). The blue curves represent the background PDF. The $\Lambda_{c}^{+}$candidates are reconstructed from (a) $p K^{-} \pi^{+}$, (b) $p K_{s}$, (c) $p K_{s} \pi^{-} \pi^{+}$, (d) $\Lambda^{0} \pi^{+}$, (e) $\Lambda^{0} \pi^{+} \pi^{-} \pi^{+}$, and (f) all five final states. 
Table 4.25: $M\left(\Lambda_{c}^{+} K_{s} \pi^{-} \pi^{+}\right)$and $M\left(\Lambda_{c}^{+} K^{-} \pi^{+} \pi^{-}\right)$mass resolutions for four-body decay modes. $\sigma_{N}$ refers to the width of the narrower of the two Gaussians; $\sigma_{W}$ refers to the wider of the two. Frac. refers to the relative fraction of the narrower Gaussian. Mean refers to the mean of the wider Gaussian. The mean of the narrow Gaussian is fixed at 0 .

\begin{tabular}{lcccc}
\hline Signal & $\sigma_{N}\left(\mathrm{MeV} / c^{2}\right)$ & $\sigma_{W}\left(\mathrm{MeV} / c^{2}\right)$ & Frac. (\%) & Mean $\left(\mathrm{MeV} / c^{2}\right)$ \\
\hline \hline$\Xi_{c}(3077)^{+}$ & $0.79 \pm 0.02$ & $2.05 \pm 0.08$ & $78 \pm 2$ & $0.52 \pm 0.07$ \\
$\Xi_{c}(3077)^{0}$ & $0.84 \pm 0.02$ & $2.41 \pm 0.07$ & $76 \pm 2$ & $0.61 \pm 0.06$ \\
$\Xi_{c}(3123)^{+}$ & $1.23 \pm 0.06$ & $2.70 \pm 0.16$ & $57 \pm 6$ & $0.10 \pm 0.07$ \\
\hline \hline
\end{tabular}

\subsubsection{Mass Resolution and Signal Efficiency}

I determine the $M\left(\Lambda_{c}^{+} K_{s} \pi^{-} \pi^{+}\right)$and $M\left(\Lambda_{c}^{+} K^{-} \pi^{+} \pi^{-}\right)$mass resolutions by studying the difference between the simulated mass and the reconstructed mass in signal-MC samples. These mass-difference distributions are fit with two Gaussian functions. The fits are shown if Figures 4.46 and 4.47 for the $\Xi_{c}(3077)^{+}$and $\Xi_{c}(3077)^{0} \mathrm{MC}$ samples, respectively. The wider Gaussians are found to have systematically higher means. The $\Xi_{c}(3123)^{+, 0} \mathrm{MC}$ distributions do not have statistically significant differences between the narrow and wide Gaussian means. I find no evidence for significant resolution differences between the five $\Lambda_{c}^{+}$decay modes. The measurements from the five $\Lambda_{c}^{+}$decay modes are averaged and listed in Table 4.25. The averaged Gaussian widths, relative means, and relative fractions are used for the double-Voigtian PDF components.

The efficiencies for finding the four-body signal decays are determined with signal-MC studies. In these studies the invariant-mass distributions for signal-MC candidates are fit with the signal PDF shapes. Because the signal MC is not distributed with a flat four-body phase-space distribution, I weight the signal-MC $\Xi_{c}(3077)^{+}$and $\Xi_{c}(3077)^{0}$ candidates to simulate the invariant-mass distributions that flat four-body phase-space distributions would have. The $M\left(\Lambda_{c}^{+} K_{s} \pi^{+} \pi^{-}\right)$and $M\left(\Lambda_{c}^{+} K^{-} \pi^{+} \pi^{-}\right)$invariant-mass ranges used in these studies are the same ranges that the signals are generated in. The fits used for estimating $\Xi_{c}(3077)^{+}$and $\Xi_{c}(3077)^{0}$ efficiencies are shown in Figures 4.48 and 4.49 , respectively. To calculate each efficiency, the number of fitted signal-MC events is divided by the weighted number of signal-MC events generated in the same invariant-mass range. All four-body efficiencies are listed in Table 4.26 with the first errors being statistical and the second errors being systematic.

As illustrated by Figure 4.50, a significant fraction of the signal PDF component is far above the simulated $\Xi_{c}(3077)^{+, 0}$ mass. Because of the lack of knowledge about signal in the higher invariant-mass range (unknown efficiencies, intermediate-resonant decays, and resolutions), the PDF component corresponding to signal is only considered below $3089 \mathrm{MeV} / c^{2}$ for $\Xi_{c}(3077)^{+}$and below $3093 \mathrm{MeV} / c^{2}$ for $\Xi_{c}(3077)^{0}$. Each upper limit creates a roughly symmetric signal region around the signal mean from three-body decay modes. The $\Xi_{c}(3123)^{+}$is far enough from the kinematic 

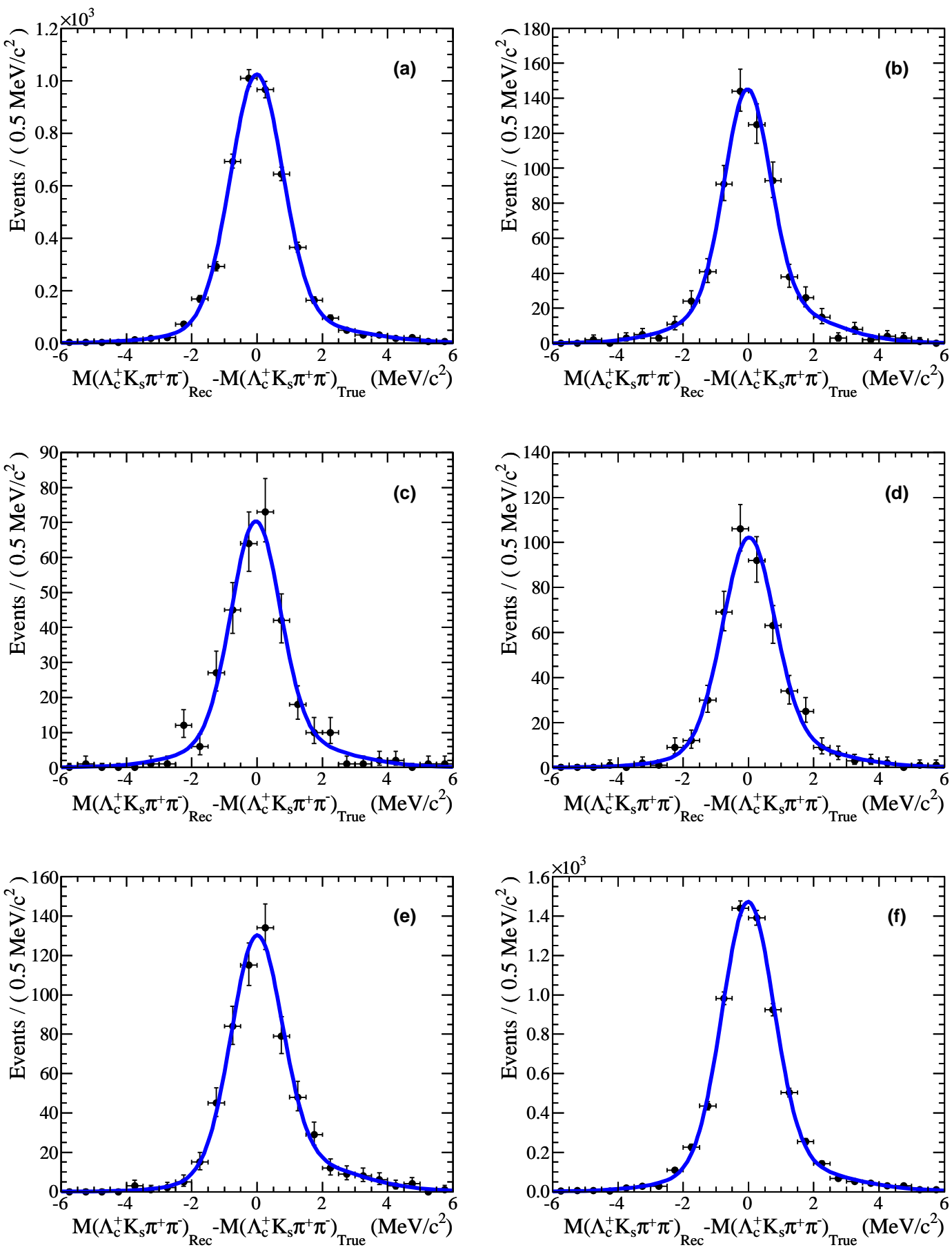

Figure 4.46: Mass resolutions for $\Xi_{c}(3077)^{+} \rightarrow \Lambda_{c}^{+} K_{s} \pi^{+} \pi^{-}$. The blue curves represent the doubleGaussian fits to the signal-MC candidates (points with error bars) where the $\Lambda_{c}^{+}$decays to (a) $p K^{-} \pi^{+}$, (b) $p K_{s}$, (c) $p K_{s} \pi^{-} \pi^{+}$, (d) $\Lambda^{0} \pi^{+}$, (e) $\Lambda^{0} \pi^{+} \pi^{-} \pi^{+}$, or (f) any of these five final states. 

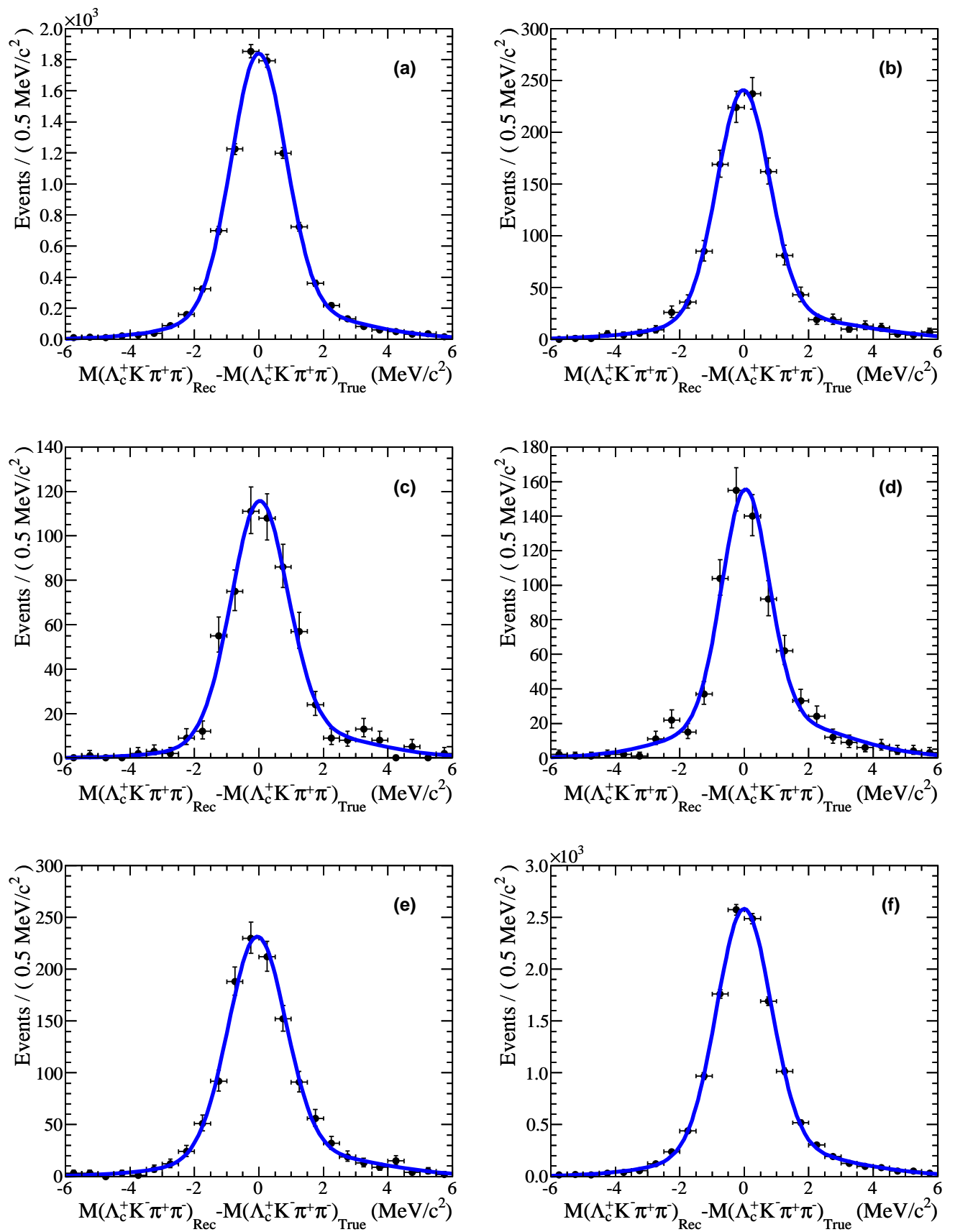

Figure 4.47: Mass resolutions for $\Xi_{c}(3077)^{0} \rightarrow \Lambda_{c}^{+} K^{-} \pi^{+} \pi^{-}$. The blue curves represent the doubleGaussian fits to the signal-MC candidates (points with error bars) where the $\Lambda_{c}^{+}$decays to (a) $p K^{-} \pi^{+}$, (b) $p K_{s}$, (c) $p K_{s} \pi^{-} \pi^{+}$, (d) $\Lambda^{0} \pi^{+}$, (e) $\Lambda^{0} \pi^{+} \pi^{-} \pi^{+}$, or (f) any of these five final states. 
Table 4.26: Estimated efficiencies, given in percent, for $\Xi_{c}(3077)^{+}, \Xi_{c}(3077)^{0}$, and $\Xi_{c}(3123)^{0}$ fourbody decay modes. Efficiencies are calculated separately for each of the five simulated $\Lambda_{c}^{+}$decay modes. The first errors are statistical and the second errors are systematic.

\begin{tabular}{lccc}
\hline Decay Mode & $\Xi_{c}(3077)^{+}$ & $\Xi_{c}(3077)^{0}$ & $\Xi_{c}(3123)^{+}$ \\
\hline \hline$p K^{-} \pi^{+}$ & $1.03 \pm 0.05 \pm 0.06$ & $5.58 \pm 0.13 \pm 0.29$ & $1.21 \pm 0.02 \pm 0.07$ \\
$p K_{s}$ & $0.34 \pm 0.03 \pm 0.03$ & $1.49 \pm 0.08 \pm 0.09$ & $0.37 \pm 0.02 \pm 0.04$ \\
$p K_{s} \pi^{-} \pi^{+}$ & $0.14 \pm 0.02 \pm 0.02$ & $0.75 \pm 0.06 \pm 0.05$ & $0.13 \pm 0.01 \pm 0.02$ \\
$\Lambda^{0} \pi^{+}$ & $0.59 \pm 0.05 \pm 0.07$ & $2.52 \pm 0.18 \pm 0.17$ & $0.52 \pm 0.03 \pm 0.06$ \\
$\Lambda^{0} \pi^{+} \pi^{-} \pi^{+}$ & $0.23 \pm 0.02 \pm 0.03$ & $1.14 \pm 0.08 \pm 0.07$ & $0.26 \pm 0.01 \pm 0.03$ \\
\hline \hline
\end{tabular}

threshold that its signal PDF component is not significantly elongated like the $\Xi_{c}(3077)^{+}$signal PDF component. Because of this, no reduced $M\left(\Lambda_{c}^{+} K_{s} \pi^{-} \pi^{+}\right)$upper limits are used for the $\Xi_{c}(3123)^{+}$ efficiency calculation, and no phase-space weighting function is used.

\subsubsection{Data Results}

Both the $M\left(\Lambda_{c}^{+} K_{s} \pi^{-} \pi^{+}\right)$and the $M\left(\Lambda_{c}^{+} K^{-} \pi^{+} \pi^{-}\right)$invariant-mass distributions do not exhibit evidence for any peaking structure. These invariant-mass distributions are shown in Figures 4.51 and 4.52, respectively. As no significant signals are found, 90\% confidence-level (CL) upper limits are determined for signal yields and their corresponding cross-sections times branching fractions. I calculate the upper limits in the same manner as the two-body decay upper limits. Again, the numbers of fitted signal are only counted for $\Xi_{c}(3077)^{+}$candidate masses below $3089 \mathrm{MeV} / c^{2}$ and for $\Xi_{c}(3077)^{0}$ candidate masses below $3093 \mathrm{MeV} / c^{2}$.

The measured $90 \%$ CL upper limit on the yield of $\Xi_{c}(3077)^{+} \rightarrow \Lambda_{c}^{+} K_{s} \pi^{-} \pi^{+}$is 1.6. As for the $\Xi_{c}(3077)^{0}$, the measured $90 \%$ CL upper limit on the yield of $\Xi_{c}(3077)^{0} \rightarrow \Lambda_{c}^{+} K^{-} \pi^{+} \pi^{-}$is 2.8. And, the measured $90 \%$ CL upper limit on the yield of $\Xi_{c}(3123)^{+} \rightarrow \Lambda_{c}^{+} K_{s} \pi^{-} \pi^{+}$is 6.5 . These yields correspond to production cross-section times branching fractions of

$$
\begin{aligned}
\sigma\left(\Xi_{c}(3077)^{+} X\right) & \times \mathcal{B}\left(\Xi_{c}(3077)^{+} \rightarrow \Lambda_{c}^{+} K_{s} \pi^{-} \pi^{+}\right) \times \mathcal{B}\left(\Lambda_{c}^{+} \rightarrow p K^{-} \pi^{+}\right)<0.4 \mathrm{fb}, \\
\sigma\left(\Xi_{c}(3077)^{0} X\right) & \times \mathcal{B}\left(\Xi_{c}(3077)^{0} \rightarrow \Lambda_{c}^{+} K^{-} \pi^{+} \pi^{-}\right) \times \mathcal{B}\left(\Lambda_{c}^{+} \rightarrow p K^{-} \pi^{+}\right)<0.1 \mathrm{fb}, \quad \text { and } \\
\sigma\left(\Xi_{c}(3123)^{+} X\right) & \times \mathcal{B}\left(\Xi_{c}(3123)^{+} \rightarrow \Lambda_{c}^{+} K_{s} \pi^{-} \pi^{+}\right) \times \mathcal{B}\left(\Lambda_{c}^{+} \rightarrow p K^{-} \pi^{+}\right)<1.4 \mathrm{fb} .
\end{aligned}
$$



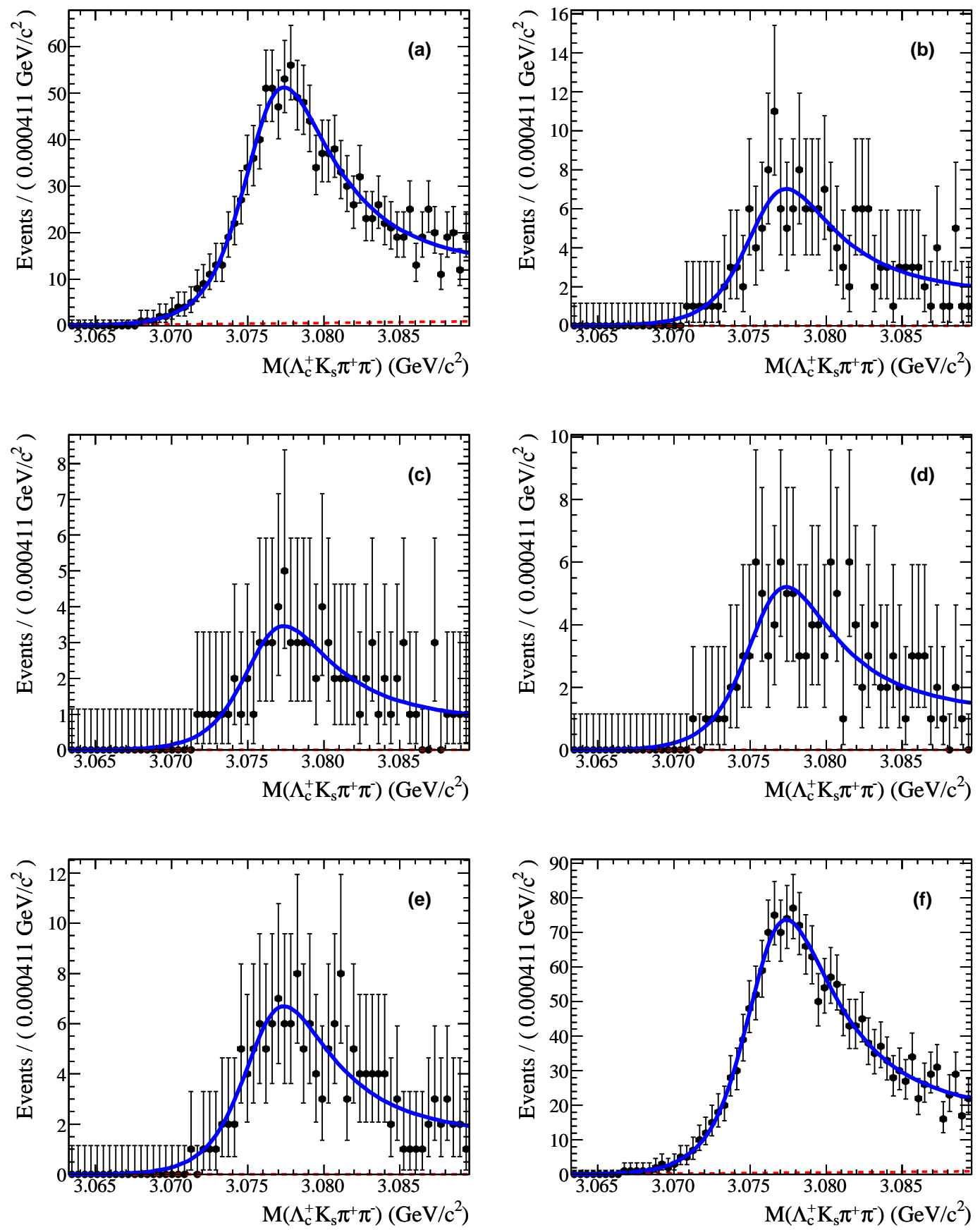

Figure 4.48: Unbinned likelihood fits to signal-MC $\Xi_{c}(3077)^{+} \rightarrow \Lambda_{c}^{+} K_{s} \pi^{+} \pi^{0}$ candidates used for estimating efficiencies. The blue curves represent the signal plus background shape fits to the signalMC samples (points with error bars) where the $\Lambda_{c}^{+}$decays to (a) $p K^{-} \pi^{+}$, (b) $p K_{s}$, (c) $p K_{s} \pi^{-} \pi^{+}$, (d) $\Lambda^{0} \pi^{+}$, (e) $\Lambda^{0} \pi^{+} \pi^{-} \pi^{+}$, or (f) any of these five final states. The red dashed lines indicate the fitted linear (or constant) background components. 

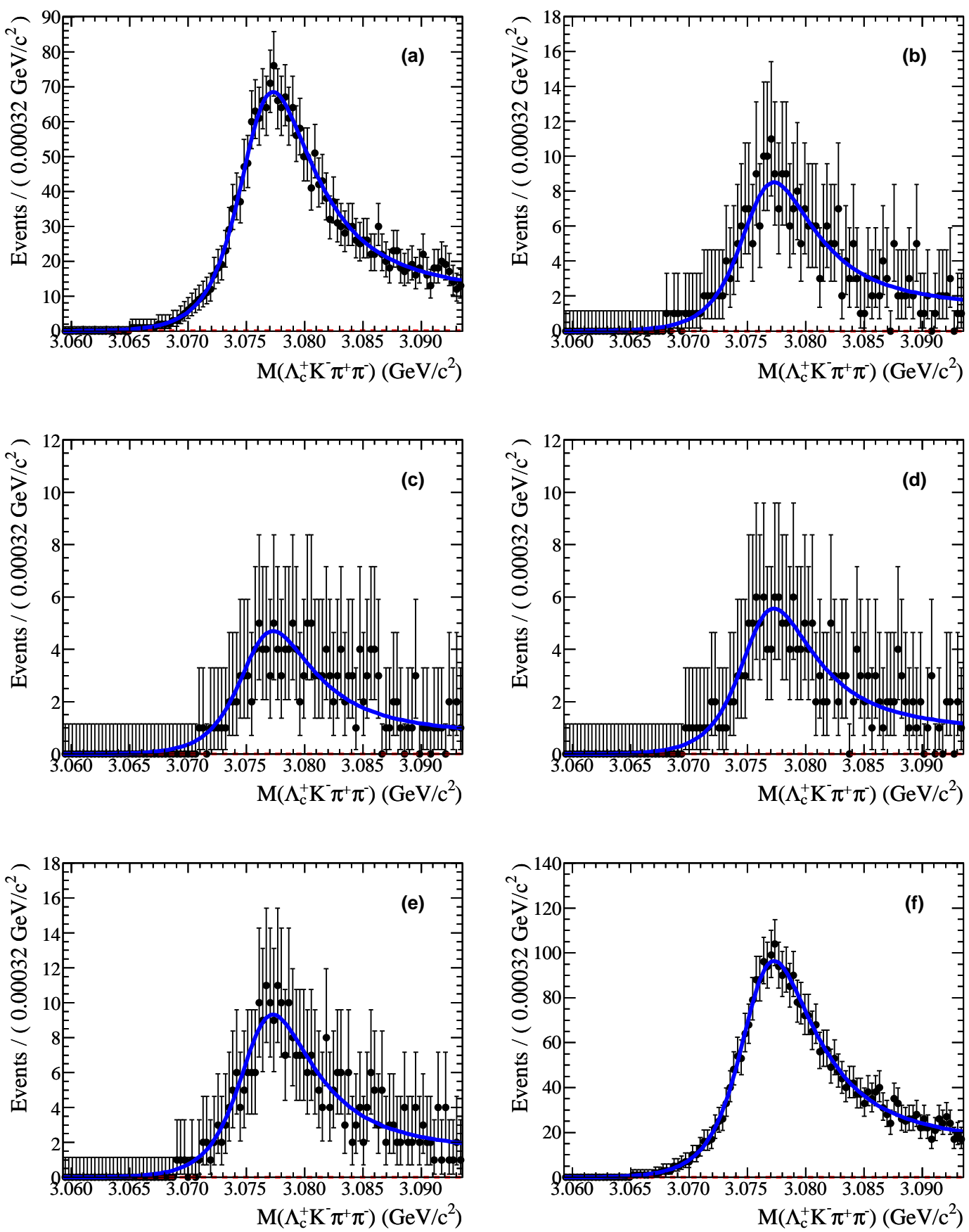

Figure 4.49: Unbinned likelihood fits to signal-MC $\Xi_{c}(3077)^{0} \rightarrow \Lambda_{c}^{+} K^{-} \pi^{+} \pi^{-}$candidates used for estimating efficiencies. The blue curves represent the signal plus background shape fits to the signalMC samples (points with error bars) where the $\Lambda_{c}^{+}$decays to (a) $p K^{-} \pi^{+}$, (b) $p K_{s}$, (c) $p K_{s} \pi^{-} \pi^{+}$, (d) $\Lambda^{0} \pi^{+}$, (e) $\Lambda^{0} \pi^{+} \pi^{-} \pi^{+}$, or (f) any of these five final states. The red dashed lines indicate the fitted linear (or constant) background components. 


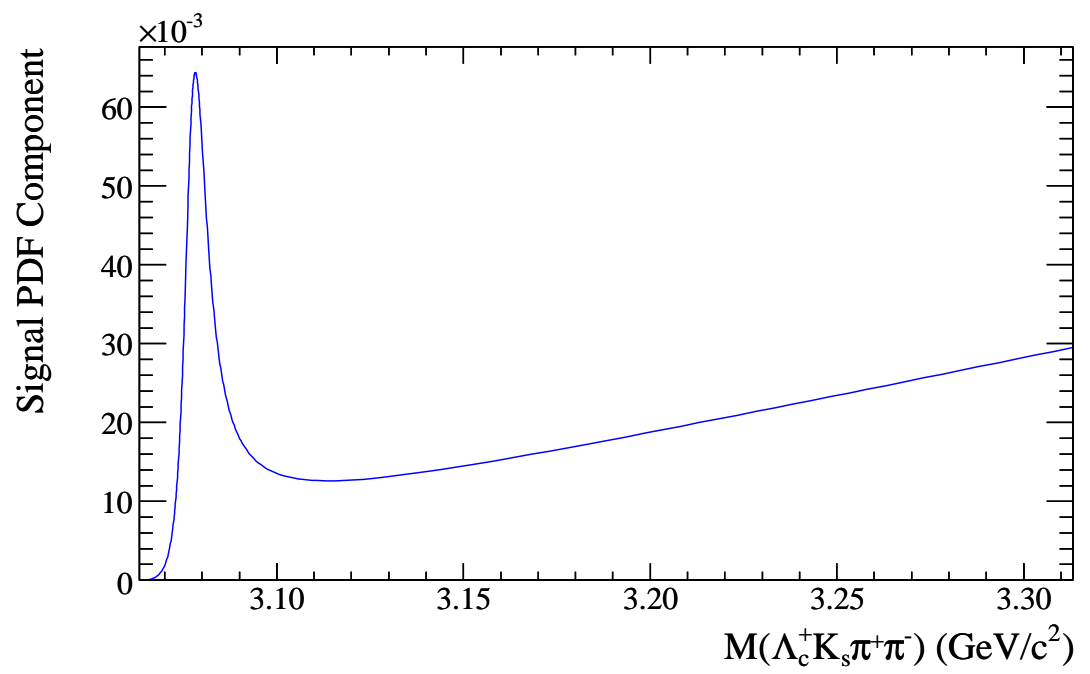

Figure 4.50: Signal PDF component for $\Xi_{c}(3077)^{+} \rightarrow \Lambda_{c}^{+} K_{s} \pi^{-} \pi^{+}$. The signal shape peaks at $3077 \mathrm{MeV} / c^{2}$. The four-body phase-space function increases more rapidy than the Breit-Wigner decreases in the region above the peak.

Also, the $90 \%$ CL upper limits on the ratios of four-body to three-body decay rates are

$$
\begin{gathered}
\frac{\mathcal{B}\left(\Xi_{c}(3077)^{+} \rightarrow \Lambda_{c}^{+} K_{s} \pi^{-} \pi^{+}\right)}{\mathcal{B}\left(\Xi_{c}(3077)^{+} \rightarrow \Lambda_{c}^{+} K^{-} \pi^{+}\right)}<0.05, \\
\frac{\mathcal{B}\left(\Xi_{c}(3077)^{0} \rightarrow \Lambda_{c}^{+} K^{-} \pi^{+} \pi^{-}\right)}{\mathcal{B}\left(\Xi_{c}(3077)^{0} \rightarrow \Lambda_{c}^{+} K_{s} \pi^{-}\right)}<0.02, \quad \text { and } \\
\frac{\mathcal{B}\left(\Xi_{c}(3123)^{+} \rightarrow \Lambda_{c}^{+} K_{s} \pi^{-} \pi^{+}\right)}{\mathcal{B}\left(\Xi_{c}(3123)^{+} \rightarrow \Lambda_{c}^{+} K^{-} \pi^{+}\right)}<0.9 .
\end{gathered}
$$

\subsection{Conclusions}

In my study of excited charm-strange baryons using the three-body decay modes $\Lambda_{c}^{+} K^{-} \pi^{+}$and $\Lambda_{c}^{+} K_{s} \pi^{-}$, I have verified the $e^{+} e^{-}$production of $\Xi_{c}(2970)^{+}$baryons (at $>9 \sigma$ statistical significance), $\Xi_{c}(3077)^{+}$baryons (at $>9 \sigma$ statistical significance), and $\Xi_{c}(3077)^{0}$ baryons (at $4.5 \sigma$ statistical significance). I measure the mass and natural width of the $\Xi_{c}(3077)^{+}$and $\Xi_{c}(3077)^{0}$ baryons and find that their masses are only $2.3 \pm 1.2 \mathrm{MeV} / c^{2}$ different and their natural widths are only 

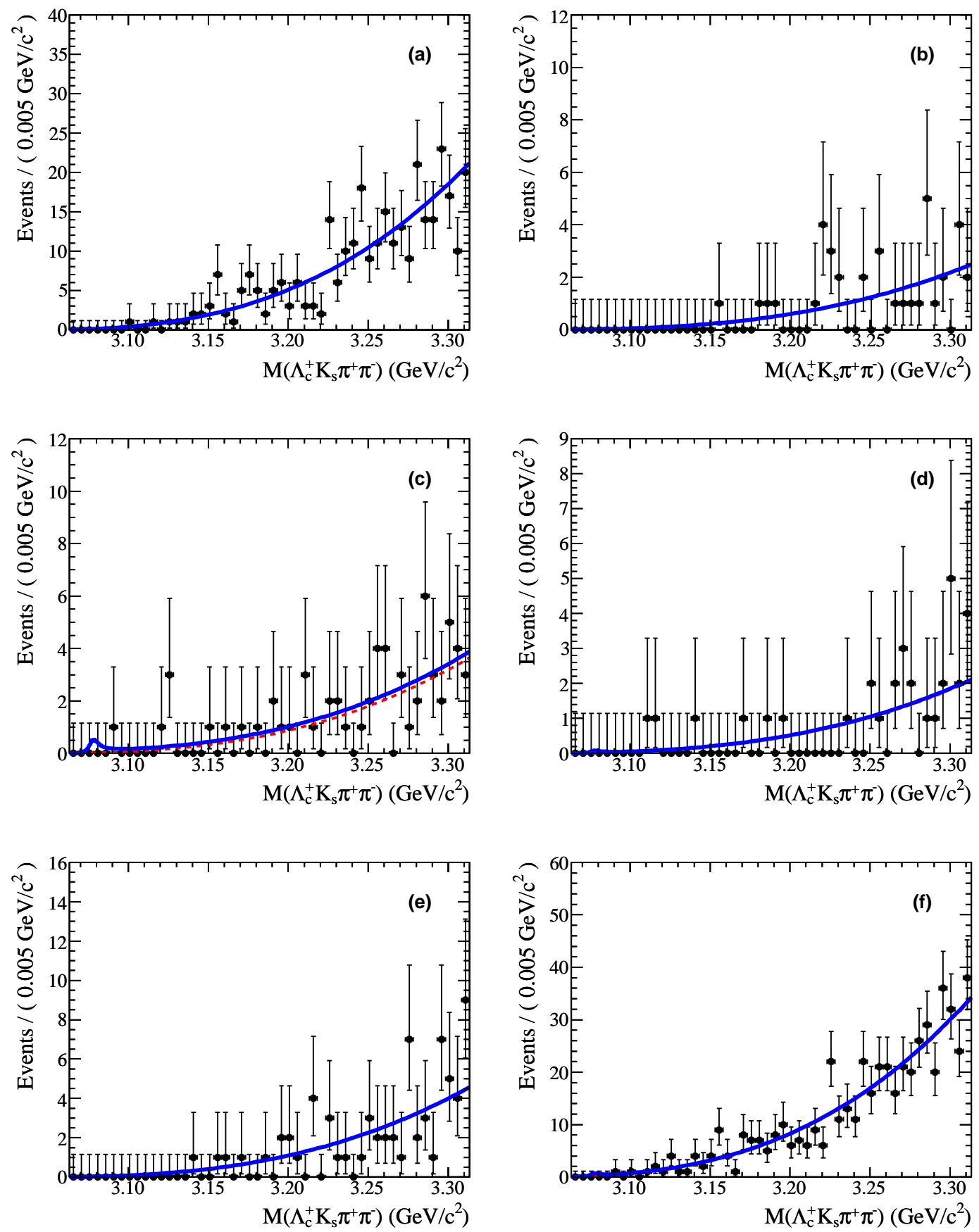

Figure 4.51: $M\left(\Lambda_{c}^{+} K_{s} \pi^{-} \pi^{+}\right)$invariant-mass distributions (points with error bars). The blue lines represent the PDF, and the dashed red lines represent the background PDF component. The $\Lambda_{c}^{+}$ is reconstructed in decays to (a) $p K^{-} \pi^{+}$, (b) $p K_{s}$, (c) $p K_{s} \pi^{-} \pi^{+}$, (d) $\Lambda^{0} \pi^{+}$, (e) $\Lambda^{0} \pi^{+} \pi^{-} \pi^{+}$, or (f) any of these five final states. 

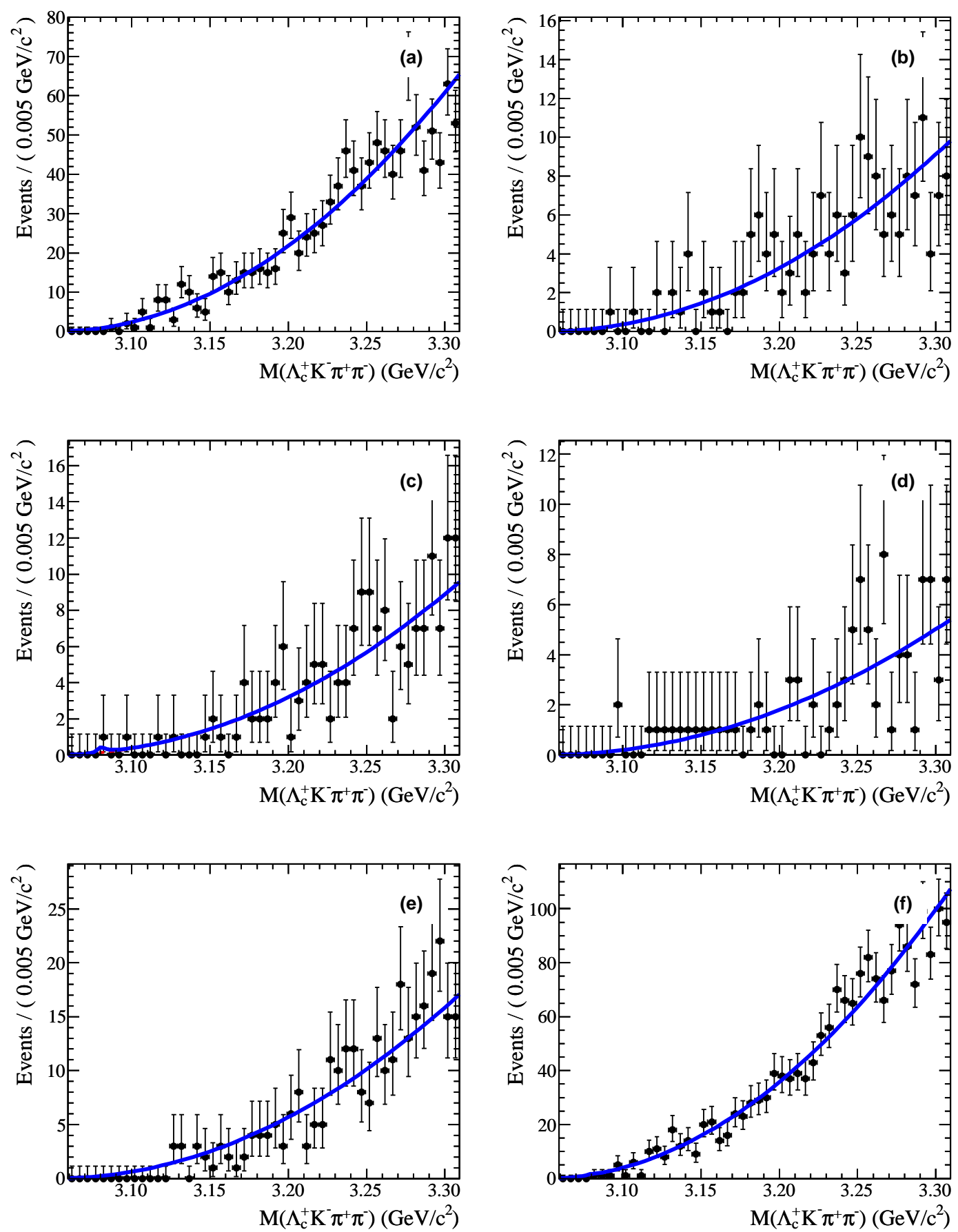

Figure 4.52: $M\left(\Lambda_{c}^{+} K^{-} \pi^{+} \pi^{-}\right)$invariant-mass distributions (points with error bars). The blue lines represent the PDF, and the dashed red lines represent the background PDF component. The $\Lambda_{c}^{+}$ is reconstructed in decays to (a) $p K^{-} \pi^{+}$, (b) $p K_{s}$, (c) $p K_{s} \pi^{-} \pi^{+}$, (d) $\Lambda^{0} \pi^{+}$, (e) $\Lambda^{0} \pi^{+} \pi^{-} \pi^{+}$, or (f) any of these five final states. 
$0.4 \pm 2.3 \mathrm{MeV} / c^{2}$ different. Their production cross-section times branching fractions are statistically consistent. These results indicate that the $\Xi_{c}(3077)^{+}$and $\Xi_{c}(3077)^{0}$ baryon states are isospin partners. The $\Xi_{c}(2970)^{+}$and $\Xi_{c}(2970)^{0}$ baryon states are also likely isospin partners, but the statistical significance of the $\Xi_{c}(2970)^{0}$ signal is only $1.7 \sigma$, making results about this state less conclusive. I measure the mass and natural width of the $\Xi_{c}(2970)^{+}$and $\Xi_{c}(2970)^{0}$ baryons and find that their masses are $3.6 \pm 4.9 \mathrm{MeV} / c^{2}$ different and their natural widths are $3.7 \pm 10.0 \mathrm{MeV} / c^{2}$ different. Their production cross-section times branching fractions are statistically consistent.

In my study of the three-body decay mode $\Lambda_{c}^{+} K^{-} \pi^{+}$, I discovered the $e^{+} e^{-}$production of the $\Xi_{c}(3055)^{+}$(at $6.4 \sigma$ statistical significance) and $\Xi_{c}(3123)^{+}$(at $3.6 \sigma$ statistical significance) baryons. These less prominent signals were discovered through the use of a two-dimensional analysis of the invariant-mass distributions $M\left(\Lambda_{c}^{+} K^{-} \pi^{+}\right)$and $M\left(\Lambda_{c}^{+} \pi^{+}\right)$. In the $M\left(\Lambda_{c}^{+} \pi^{+}\right)$distribution I observe signals for $\Sigma_{c}(2455)^{++}$and $\Sigma_{c}(2520)^{++}$resonances. I find that the $\Xi_{c}(3055)^{+}$and $\Xi_{c}(3123)^{+}$ baryons decay through two-body intermediate states $\Sigma_{c}(2455)^{++} K^{+}$and $\Sigma_{c}(2520)^{++} K^{+}$, respectively. I do not observe statistically significant signals for the states $\Xi_{c}(3055)^{0}$ and $\Xi_{c}(3123)^{0}$, which would be isospin partners to the states $\Xi_{c}(3055)^{+}$and $\Xi_{c}(3123)^{+}$. I determine $90 \%$ confidence-level upper limits for the production cross-section times branching fractions for $\Xi_{c}(3055)^{0}$ and $\Xi_{c}(3123)^{0}$ baryons; these upper limits do not rule out the production of $\Xi_{c}(3055)^{0}$ and $\Xi_{c}(3123)^{0}$ baryons at similar rates as their isospin partners.

For each baryon state for which I measure a statistically significant signal, I search for their decays to the two-body final states $\Lambda_{c}^{+} K_{s}$ and $\Lambda_{c}^{+} K^{-}$, and their decays to the four-body final states $\Lambda_{c}^{+} K_{s} \pi^{-} \pi^{+}$and $\Lambda_{c}^{+} K^{-} \pi^{+} \pi^{-}$. I find no statistically significant signals for decays to these two-body and four-body final states. I measure $90 \%$ confidence-level upper limits for the production crosssection times branching fractions for each of these cases. Using these upper limits, I determine upper limits on two-body and four-body branching ratios with respect to the measured three-body branching fractions. The upper limits on the two-body to three-body branching ratios are on the order of 1 , and the upper limits on the four-body to three-body branching ratios are as low as $2 \%$. 


\section{Chapter 5}

\section{Summary}

I have described my study of both double-charm and charm-strange baryons produced from $e^{+} e^{-}$ annihilations in the BABAR Detector. This study was motivated by the desire to experimentally observe new baryon states that test the basic models of baryonic bound states. The observation of double-charm baryons would herald the first opportunity to study in the same bound state both the interaction of two heavy quarks (charm) and the interaction of heavy quarks with a light quark (up or down). The observations of several excited charm-strange baryon states in my analysis provides an expanded scientific picture for the better understanding of the dynamics in baryon states with both excitations and heavier quarks.

I designed my double-charm baryon analysis as both a follow up to observationa in a hadroproduction experiment and as an independent search for double-charm baryons within a wide mass range. The $210 \mathrm{MeV} / c^{2}$-wide double-charm baryon search region was blinded during the process of optimizing the selection criteria for double-charm baryon discovery. Despite finding a large number of single charm baryons, my analysis find no evidence for double-charm baryons. I further analyzed the data to determine upper limits on the product of double-charm baryon production cross-section and the branching fractions in the decay chain. Relative to the production cross-section for $\Lambda_{c}^{+}$ baryons, the resultant $95 \%$ confidence-level upper limits are at most $0.1 \%$. These results cast some doubt on other experimental claims of high rates of double-charm baryon production relative to $\Lambda_{c}^{+}$ production.

The analysis of excited charm-strange baryons was designed to substantiate another $e^{+} e^{-}$experiment's claim of the discovery of three new baryons: $\Xi_{c}(2970)^{+}, \Xi_{c}(3077)^{+}$, and $\Xi_{c}(3077)^{0}$. I observe these three states with my analysis of the BABAR data set. During the process, I discovered two additional excited charm-strange baryons: $\Xi_{c}(3055)^{+}$and $\Xi_{c}(3123)^{+}$. These discoveries were predicated on my use of a two-dimensional fit to the data, which helped to separate signal distributions from background. This analysis technique also helps to accurately measure the mass and width of the $\Xi_{c}(2970)^{+}$signal, which is near its kinematic limits. 
The BABAR Collaboration continues to record more $e^{+} e^{-}$annihilations, providing the possibility that more sensitive studies of double-charm and charm-strange baryons may be performed in the future. It is also possible that these baryons might be observed in their decays to other final states such as $p D^{+} K^{-}$and $\Xi_{c}^{+} \pi^{-} \pi^{+}$. Determining the relative decay rates and decay modes of the excited charm-strange baryons observed in this study would help to determine the excitations of these states. It is my hope that my analytical work presented here will provide support for such future endeavors. 


\section{Bibliography}

[1] M. Mattson et al. [SELEX Collaboration], "First observation of the doubly charmed baryon $\Xi_{c c}^{+}$, Phys. Rev. Lett. 89, 112001 (2002) [arXiv:hep-ex/0208014].

[2] M. A. Moinester et al. [SELEX Collaboration], "First observation of doubly charmed baryons," Czech. J. Phys. 53, B201 (2003) [arXiv:hep-ex/0212029].

[3] A. Ocherashvili et al. [SELEX Collaboration], "Confirmation of the double charm baryon $\Xi_{c c}(3520)^{+}$via its decay to $p D^{+} K^{-}, "$ Phys. Lett. B 628, 18 (2005) [arXiv:hep-ex/0406033].

[4] B. Aubert et al. [BABAR Collaboration], "Search for doubly charmed baryons $\Xi_{c c}^{+}$and $\Xi_{c c}^{++}$ in BABAR," Phys. Rev. D 74, 011103 (2006) [arXiv:hep-ex/0605075].

[5] C. Riccardi [FOCUS Collaboration], "Charmed baryons and heavy quark spectroscopy results from FOCUS," Prepared for 31st International Conference on High Energy Physics (ICHEP 2002), Amsterdam, The Netherlands, 24-31 Jul 2002.

[6] R. Chistov et al. [BELLE Collaboration], "Observation of new states decaying into $\Lambda_{c}^{+} K^{-} \pi^{+}$ and $\Lambda_{c}^{+} K_{s}^{0} \pi^{-}, "$ Phys. Rev. Lett. 97, 162001 (2006) [arXiv:hep-ex/0606051].

[7] W. M. Yao et al. [Particle Data Group], "Review of particle physics," J. Phys. G 33, 1 (2006).

[8] B. Aubert et al. [BABAR Collaboration], "Observation of an excited charm baryon $\Omega_{c}^{*}$ decaying to $\Omega_{c}^{0} \gamma, "$ Phys. Rev. Lett. 97, 232001 (2006) [arXiv:hep-ex/0608055].

[9] B. Aubert et al. [BABAR Collaboration], "Observation of a charmed baryon decaying to $D^{0} p$ at a mass near $2.94 \mathrm{GeV} / c^{2}$," Phys. Rev. Lett. 98, 012001 (2007) [arXiv:hep-ex/0603052].

[10] B. Aubert et al. [BABAR Collaboration], Nucl. Instrum. Methods Phys. Res., Sect. A 479, 1 (2002).

[11] B. Guberina, B. Melic, and H. Stefancic, "Inclusive decays and lifetimes of doubly charmed baryons," Eur. Phys. J. C 9, 213 (1999) [Eur. Phys. J. C 13, 551 (2000)] [arXiv:hep-ph/9901323]. 
[12] V. V. Kiselev, A. K. Likhoded, and A. I. Onishchenko, "Lifetimes of doubly charmed baryons: $\Xi_{c c}^{+}$and $\Xi_{c c}^{++}, "$ Phys. Atom. Nucl. 62, 1940 (1999) [Yad. Fiz. 62, 2095 (1999)].

[13] S. Migura, D. Merten, B. Metsch, and H. R. Petry, "Charmed baryons in a relativistic quark model," Eur. Phys. J. A 28, 41 (2006).

[14] H. Garcilazo, J. Vijande, and A. Valcarce, "Faddeev study of heavy baryon spectroscopy," J. Phys. G 34, 961 (2007).

[15] H. Y. Cheng and C. K. Chua, "Strong decays of charmed baryons in heavy hadron chiral perturbation theory," Phys. Rev. D 75, 014006 (2007).

[16] J. L. Rosner, "Hadron spectroscopy: Theory and experiment," J. Phys. G 34, S127 (2007) [arXiv:hep-ph/0609195].

[17] C. Chen, X. L. Chen, X. Liu, W. Z. Deng and S. L. Zhu, "Strong decays of charmed baryons," [arXiv:0704.0075 (hep-ph)].

[18] C. Bozzi et al. [BABAR Collaboration], "The BaBar silicon vertex tracker," Nucl. Instrum. Meth. A 461, 162 (2001).

[19] G. Sciolla et al. [BABAR Drift Chamber Collaboration], "The BABAR Drift Chamber," Nucl. Instrum. Meth. A 419, 310 (1998).

[20] I. Adam et al. [BABAR-DIRC Collaboration], "The DIRC particle identification system for the BaBar experiment," Nucl. Instrum. Meth. A 538, 281 (2005).

[21] T. Sjostrand, "High-energy physics event generation with PYTHIA 5.7 and JETSET 7.4," Comput. Phys. Commun. 82, 74 (1994).

[22] D. J. Lange, "The EvtGen particle decay simulation package," Nucl. Instrum. Methods Phys. Res., Sect. A 462, 152 (2001).

[23] S. Agostinelli et al. [GEANT4 Collaboration], "GEANT4: A simulation toolkit," Nucl. Instrum. Methods Phys. Res., Sect. A 506, 250 (2003).

[24] V. V. Kiselev, A. K. Likhoded, and M. V. Shevlyagin, "Double charmed baryon production at B factory," Phys. Lett. B 332, 411 (1994) [arXiv:hep-ph/9408407].

[25] V. V. Braguta, V. V. Kiselev, and A. E. Chalov, "Pair production of doubly heavy diquarks," Phys. Atom. Nucl. 65, 1537 (2002).

[26] J. P. Ma and Z. G. Si, "Factorization approach for inclusive production of doubly heavy baryon," Phys. Lett. B 568, 135 (2003) [arXiv:hep-ph/0305079]. 
[27] S. S. Gershtein, V. V. Kiselev, A. K. Likhoded, and A. I. Onishchenko, "Spectroscopy of doubly charmed baryons: $\Xi_{c c}^{+}$and $\Xi_{c c}^{++}$," Mod. Phys. Lett. A 14, 135 (1999) [arXiv:hep-ph/9807375].

[28] R. Roncaglia, D. B. Lichtenberg, and E. Predazzi, "Predicting the masses of baryons containing one or two heavy quarks," Phys. Rev. D 52, 1722 (1995) [arXiv:hep-ph/9502251].

[29] J. Vijande, H. Garcilazo, A. Valcarce, and F. Fernandez, "Spectroscopy of doubly charmed baryons," Phys. Rev. D 70, 054022 (2004) [arXiv:hep-ph/0408274].

[30] S. P. Tong, Y. B. Ding, X. H. Guo, H. Y. Jin, X. Q. Li, P. N. Shen and R. Zhang, "Spectra of baryons containing two heavy quarks in potential model," Phys. Rev. D 62, 054024 (2000) [arXiv:hep-ph/9910259].

[31] J. G. Korner and H. W. Siebert, "Charm baryons: Theory and experiment," Ann. Rev. Nucl. Part. Sci. 41, 511 (1991).

[32] James D. Bjorken, "Estimates of decay branching ratios for hadrons containing charm and botton quarks," Unpublished (1986).

[33] B. Aubert et al. [BABAR Collaboration], "Measurement of double charmonium production in $e^{+} e^{-}$annihilations at $\sqrt{s}=10.6 \mathrm{GeV}, "$ Phys. Rev. D 72, 031101 (2005) [arXiv:hep-ex/0506062].

[34] C. Albertus, J. E. Amaro, E. Hernandez, and J. Nieves, "Charmed and bottom baryons: A variational approach based on heavy quark symmetry," Nucl. Phys. A 740, 333 (2004) [arXiv:nucl-th/0311100].

[35] A. G. Frodesen, O. Skjeggestad, and H. Tofte, "Probability and Statistics in Particle Physics," Universitetsforlaget 1979, pp. 252-255, ISBN 82-00-01906-3.

[36] R. D. Cousins and V. L. Highland, "Incorporating systematic uncertainties into an upper limit," Nucl. Instrum. Meth. A 320, 331 (1992).

[37] B. Aubert et al. [BABAR Collaboration], "A precision measurement of the $\Lambda_{c}^{+}$baryon mass," Phys. Rev. D 72, 052006 (2005) [arXiv:hep-ex/0507009].

[38] L. Lyons, D. Gibaut, and P. Clifford, "How to Combine Correlated Estimates of a Single Physical Quantity," Nucl. Instrum. Meth. A 270, 110 (1988). 